


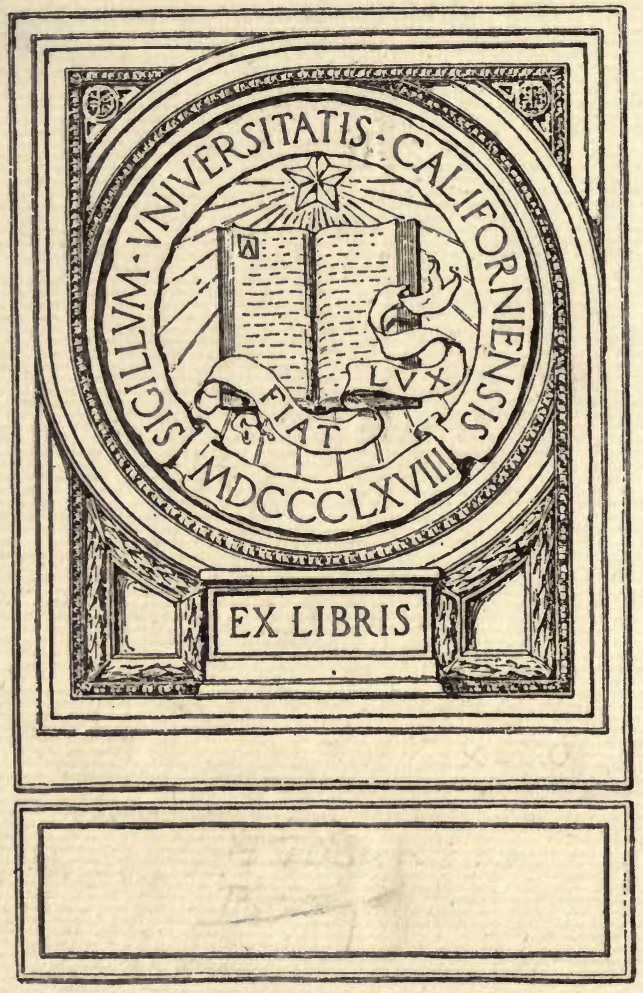






\section{PRACTICAL PHOTO-MICROGRAPHY}




\section{PRACTICAL}

\section{PHOTO-MICROGRAPHY}

BY

J. EDWIN BARNARD, F.R.M.S.

LECTURER IN MICROSCOPY, KING'S COLLEGE, LONDON

ILLUSTRATED

LONDON

EDWARD ARNOLD

1911 
oft 25

B35

$$
\begin{aligned}
& \because \because \vdots \quad \because \quad \vdots \vdots \vdots \vdots \vdots
\end{aligned}
$$

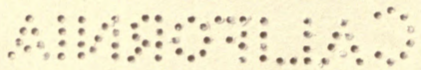




\section{PREFA CE}

IN the following pages an attempt has been made to set out the methods of obtaining photographs of microscopic objects. It is not an easy matter to describe the details and processes of a practical scientific subject in a lucid and interesting manner: it is one thing to be conversant with the practical side of the subject, but quite another to write such a description of the procedure as will enable another person to carry it out. In the present instance all that is claimed is that the attempt is a straightforward one, founded on practical experience.

The description of instruments is as full as the circumstances would appear to justify ; that, for instance, of the microscope, about which much can be learned from books on pure microscopy, is less full than the description of cameras and their construction. The number of references to the work of others is small : not because the writer wishes in any way to pass over such work or to minimise its importance, but because the subject is one that is best treated in the light of personal experience.

Sincere thanks are due to Professor R. T. Hewlett, who, in addition to reading the proofs and making valuable suggestions thereon, has at all times given advice, and placed at the author's disposal the benefit of his wide experience. Thanks are also due to Mr. F. Shillington Scales, M.A. ; and to Mr. G. Beilby, F.R.S., and Mr. C. O. Bannister for the loan of blocks and specimens. Particularly, also, should be mentioned the author's indebtedness to $\mathrm{Mr}$. Conrad Beck, whose Cantor lectures on "The Theory of 
the Microscope' constitute one of the most lucid expositions of the subject which is available, and from which some illustrations, and many suggestions in the text have been taken.

Lastly, to the various members of the optical trade, both English and Continental, from whom a number of illustrations have been borrowed, thanks are also tendered. In all cases these have been readily supplied, and information has been given without stint. The author ventures to express the hope that the book may be of assistance to some struggling with the initial difficulties of the subject; and that it may induce others to enter a field of work which is still not fully explored, and which has almost infinite possibilities, whether considered as a study in itself, or as a means of placing on record the work of others.

J. E. B.

London: March 1911. 


\title{
CONTENTS
}

\author{
CHAPTER I
}

PAGES

INTRODUCTORY

Photography, its field and how to enter upon it . . . . 1-4

\section{CHAPTER II}

THE MICROSCOPE

Its Mechanical Parts-The Forms best suited for the Purpose in View-Some Typical Instruments . . . . . 5-17

\section{CHAPTER III}

THE OPTICAL EQUIPMENT: OBJECTIVES AND OCULARS

Objectives-Dry- and Immersion-lenses-Achromatic and Apochromatic Systems-Penetration-Flatness of Field-Testing of Objectives - Resolving Power-Objective-changers - The Ocular or Eye-piece, Ordinary and Compensating-Projectionoculars

CHAPTER IV

THE OPTICAL EQUIPMENT (continued): CONDENSERS AND COLLECTING-LENSES

The Mirror-Abbe Condensers-Achromatic and Oil-immersion Condensers-The Aplanatic Cone-Dark-ground Illuminators -Illuminators for Opaque Objects, Vertical and Oblique Illuminators-Auxiliary Collecting-lenses . . . . 46-74

\section{CHAPTER V \\ SOURCES OF ILLUMINATION}

Oil Lamps-Welsbach Gas-Acetylene-Generators for AcetyleneLime-light-Electrical Illuminants-Nernst Lamp-Mercuryvapour Lamp-The Electric-arc-Sunlight . . . 75-110 vii 


\section{CHAPTER VI}

THE PHOTO-MICROGRAPHIC CAMERA

PAGES

Simple Forms of Vertical Cameras-Van Heurck's ApparatusJ. W. Gordon's Method-Types of Horizontal Cameras by Swift, Zeiss, Ross, Bausch and Lomb-Camera on the Geometric Slide Principle-Combined Vertical and Horizontal Cameras-Cameras for Instantaneous Work and for Opaque Objects . . 111-145

\section{CHAPTER VII}

\section{THE USE AND MANIPULATION OF THE MICROSCOPE}

Setting up an Object and obtaining a properly Illuminated Field of View-Focussing the Objective-The Selection of a Suitable Ocular-The Care of Objectives and Oculars-Care of the EyesCorrection for Thickness of Cover-glass and Adjustment of Tube length-The Adjustment of the Sub-stage Condenser-The Adjustment of the Auxiliary Collecting-lens . . 146-174

\section{CHAPTER VIII}

\section{GENERAL PRELIMINARY PREPARATIONS}

Vibration and its Prevention-Light-excluders and Methods of Connection between Microscope and Camera-Diffused LightHeat from Illuminant - Diaphragms - Size of Camera Focussing-screens-Focussing Eye-pieces-Placing Microscope in Relation to Camera-Methods of Exposing-The Process of Focussing-Magnification-The Dark-room and its Illumination

\section{CHAPTER IX}

\section{COLOUR-SCREENS-PLATES-EXPOSURE}

Colour-screens for correcting Objectives-To increase and decrease Colour Contrast in the Object-Methods of making Gelatin and Collodion Colour-screens-Fluid-screens-The Absorptionspectra of some Dyes used for Colour-screens-Monochromatic Light-apparatus-Screens for Use with Mercury-vapour Lamps - Photographic Plates and the Influence of Speed on Size of Grain-Suitable Ordinary and Isochromatic Plates-Halation and its Prevention-The Resolution of Photographic PlatesExposure and its Estimation

205-228

\section{CHAPTER X}

\section{PHOTOGRAPHIC PROGESSES}

The Development of the Negative-Developers-Defects in Negatives -Intensification and Reduction-Printing Processes-P.O.P. and Bromide Prints-Glazing and Enamelling Prints-Lanternslides on Gelatin and Wet-collodion Plates-Their Development and After-treatment-The Projection of Lantern-slides . 


\section{CHAPTER XI}

\section{SOME SPECIAL PROCESSES}

PAGES

Photo-micrography by means of Ultra-violet Light-The Photography of Metals and Similar Opaque Objects-The Photography of Tube- and Plate-cultures of Bacteria-Stereoscopic Photomicrography-The Production of Lantern-slides in Colour by the Sanger-Shepherd Three-colour Method-The Production of Lantern-slides by the 'Autochrome,' 'Thames' and Dufay Colour Processes

\section{CHAPTER XII}

SOME PROGRESSIVE EXAMPLES

Description of Plates I-X

\section{APPENDICES}

I. Microscopic and Spectroscopic Standards for Measurement of Length . . . . . . . . . 305

II. Weights and Measures . . . . . . . . 305

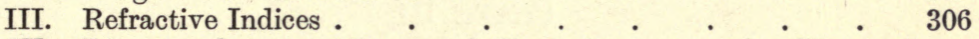

IV. Distance between Lens and Focussing-screen for Given Enlargement or Reduction . . . . . . 306

V. Determination of N.A. of Objectives . . . . . . 307

VI. Bibliography . . . . . . . . . 310 


\title{
LIST OF PLATES
}

\author{
AT END OF BOOK
}

\section{PLATE I}

1. Transverse Section of Stem of Clematis. $\times 20$

2. Transverse Section of Stem of Beech. $\times 20$

3. Proboscis of Blow-fly. $\times 100$

\section{PLATE II}

1. Group of Foraminifera. $\times 25$

2. Transverse Section of Stem of Sarsaparilla. $\times 50$

3. Spine of an Echinus. $\times 40$

4. Section of Human Scalp. $\times 75$

\section{PLATE III}

1. Transverse Section of Human Tongue. $\times 50$

2. Transverse Section of Stem of Black Pepper. $\times 30$

3. Group of Foraminifera. $\times 25$

4. Medulla of Monkey. $\times 15$

\section{PLATE IV}

1. Impression Preparation of Bacillus anthracis. $\times 100$

2. Colony of Bacillus coli communis. $\times 50$

3. Group of Mixed Diatoms. $\times 100$

4. Surface Colony of Ringworm Organism. $\times 2$

5. Surface Colony of Bacillus anthracis. $\times 20$

\section{PLATE V}

l. Tripanosoma gambiense. $\times 450$

2. Diatom, Orthonies splendida. $\times 500$

3. Tripanosoma gambiense. $\times 1000$

4. Cover-glass Preparation of Pigeon's Blood. $\times 360$

5. Diatom, Navicula lyra. $\times 600$

6. Unstained Living Yeast-cells. $\times 500$

\section{PLATE VI}

1. Actinomycosis bovis. Section of Tongue of Cow. $\times 350$

2. Bacillus pestis in Blood (Bubonic Plague). $\times 750$

3. Bacillus tuberculosis, Giant Cell in Lung. $\times 1000$

4. Koch's Spirillum of Cholera. $\times 1500$

5. Human Hair showing Ringworm Organism. $\times 450$ 


\section{PLATE VII}

1. Bacillus anthracis. Cover-glass Preparation of Blood of Guinea-pig. $\times 750$

2. Spirochote pallida. $\times 1500$

3. Streptococcus pyogenes. $\times 1250$

4. Bacillus typhosus. Cover-glass Preparation showing Flagella. $\times 1250$

5. Micrococcus gonorrhoea. Cover-glass Preparation of Pus. $\times 1500$

6. Bacillus of Leprosy in Skin. $\times 1250$

\section{PLATE VIII}

1. Diatom, Navicula lyra. $\times 850$

2. Podura Scale. (Lepidocyrtus curvicollis). $\times 1750$

3. Podura scale. (Lepidocyrtus curvicollis). $\times 2500$

4. Diatom, Pleurosigma angulatum. $\times 1000$

\section{PLATE IX}

1. Diatom, Amphipleura pellucida. $\times 1500$

2-5. Diatoms, Navicula rhomboides. $\times 750$

6. Diatom, Amphipleura pellucida. $\times 1500$

\section{PLATE $\mathbf{X}$}

1. Metal Surface, of Tin and Antimony. $\times 20$

2. Metal Surface, of Silver and Copper. $\times 250$

3. Cemented Steel, showing Carbide of Iron. $\times 100$

4. Metal surface, Manganese Sulphide in Steel. $\times 1000$

\section{LIST OF ILLUSTRATIONS}

FIG. PAGE

1. Wales type of Microscope (Swift) . . . . . $\quad$ - 10

2. Zeiss Microscope with Photo-micrographic Stage . . $\quad$ - 12

3. Van Heurck Microscope (Watson) . . . . 13

4. Leitz Stand, $1 a$ with English Foot $\quad$. $\quad$. $\quad$. $\quad$. $\quad 15$

5. D.P.H. Microscope (Baker) . $\quad$. $\quad . \quad$. $\quad . \quad . \quad 16$

6. Effect of Oil Immersion compared with Dry Lens . $\quad$ - 20

7. Increased Penetration due to stopping-down of Objective $\quad 23$

8. Photographs of Abbe Test-plate . . . . . . 31

9. Apochromatic Objective with Correction-collar (Zeiss) . $\quad$. 33

10. Sliding Objective-changer (Zeiss) . . . . . . 37

11. Compensating Eye-pieces (Zeiss) . $\quad . \quad$ • $\quad . \quad$. $\quad . \quad 39$

12. Projection Eye-pieces . • . . . . . . 44

13. Correction for Spherical Aberration . . . . . 48

14. Zeiss Centreing-collar used as Condenser $\quad$. $\quad . \quad$. $\quad 50$

15. Path of Rays in Paraboloid Condenser . . . . . $\quad$. 57

16. Frosted Silver-leaf Film on Glass . . . . . $\quad$ - 62

17. Reflecting Illuminator for Low Powers . $\quad$. $\quad$ • $\quad$ - 66

18. Vertical Illuminator for Opaque Objects (Watson) . . . 66

19. " " " " " $"$ (Leitz) . . . 69

20. Path of Rays in Watson's Subsidiary Achromatic Lens ·. . 71

21. Collecting-lens Systems . . . . . . . . 72

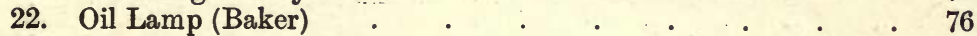

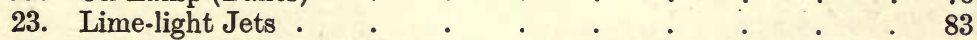

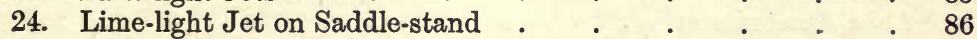

25. Adjustable Lime-light Tray (Gwyer Jet Co.) • . 87

26. Nernst Electric 'Luna' Burner . . . , , , 89 
27. Mercury-vapour Lamp (Baker)

28. " , " (Swift) . . . . . . 95

29. Correct Position of Electric-arc Carbons _ . $\quad$ • $\quad$ - 97

30. Electric-arc Carbons under Different Conditions . . $\quad 98$

31. Hand-feed Arc Lamp . . . . . . . . 100

32. $"$ ",$\quad . \quad . \quad . \quad . \quad . \quad . \quad . \quad .102$

33. Method of connecting-up Arc Lamp . . . . 105

34. Leitz hand-feed Arc Lamp . . . . . . . . 106

35. Simple form of Heliostat . $\quad$. $\quad . \quad$. $\quad . \quad$. 109

36. " ", , Photo-micrographic Camera (Swift) . 112

37. Vertical Camera (Zeiss) . . . . . . $\quad$. 113

38A. Combined Horizontal and Vertical Camera (Leitz) . 114

38в. " " " " " " $"$ (Leitz) . . 115

39. $", \quad " \quad " \quad$ " $"$ (Watson) . . 116

40. Van Heurck's Vertical Camera . . . . . . . 118

41. Ordinary Camera used as Vertical Camera . . . $\quad . \quad 120$

42. Simple form of Horizontal Camera (Swift) . . . 121

43. Students' form of Horizontal Camera (Watson) . . . 122

44. Horizontal Camera . . . . . . . . 125

45. Method of Controlling Fine-adjustment $\quad$. $\quad$. $\quad$. $\quad 126$

46. Large Micrographic Camera (Zeiss) $\quad$ × $\quad . \quad$. $\quad . \quad$. 127

47. Author's Photo-micrographic Apparatus . . . . $\quad$. 131

$48 . \quad$ 4. " " $"$. . . . 133

49. Geometric Slide . • . . . . . . . 134

50. Combined Horizontal and Vertical Camera (Zeiss) . . 136

51. " " " , " . . . 137

52. Reflex Camera "in combination with Van Heurck Vertical $\quad$. 139

53. Camera for Metallography (Leitz) . . . . . 142

54. Path of Rays in Vertical Illuminator (Leitz) . . . . 144

55. Influence of Cover-glass Thickness on Adjustment . . $\quad$. 155

56. , , " , , , , " . 157

57. Effect of varying Cover-glass Thickness. " . . facing p. 158

58. Path of Rays in Sub-stage Condenser . . . . . 166

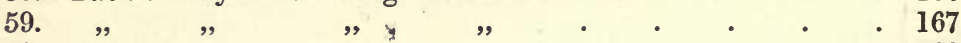

60. " " " $"$ " $"$. . . . 168

61. Supporting Camera to minimise Vibration . . . . . 177

62. Appliance for excluding Light $\quad . \quad$. $\quad . \quad$. $\quad . \quad$. 178

63. Focussing-glass (Zeiss) $\quad$. $\quad . \quad$. $\quad . \quad . \quad . \quad 186$

64. Improvised Glass Cell for Screen . $\quad . \quad$. $\quad . \quad$. 207

65. Monochromatic Trough . . . . . . 208

66. Kingsford's Trough for Fluid Screens (Watson) — . $\quad$ • 209

67. Spectra of Various Dyes and Colour Screens . . facing p. 210

68. Monochromatic Light Apparatus (Baker) . . . . 215

69. Grain of Photographic Plates . . . . facing p. 220

70. Path of Rays in Vertical Illuminator . . . . . 257

71. Martens' Photo-micrographic Stand . . . . . 258

72. Rosenhain's Metallurgical Microscope (Beck) . . . . 260

73. Engineers' Metallurgical Microscope (Swift) . . . $\quad$. 262

74. Arrangement of Camera for photographing Bacteria . $\quad 270$

75. Plate Culture of Bacteria . . . . facing p. 270

76. $", \quad$ " $"$. . . . . . . . 270

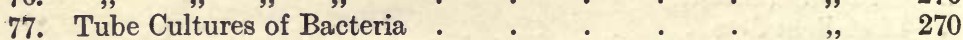

78. Abbe Apertometer . . . . . . . . . . . . . 308

79. Cheshire , . . . . . . 309 


\section{PRACTICAL PHOTO-MICROGRAPHY}

\section{CHAPTER I}

\section{INTRODUCTORY}

As an instrument of research, either in its application to scientific work, or in its varied uses where scientific methods have been applied industrially, the microscope now holds a pre-eminent position. It was early recognised that some means of recording the varied appearances of microscopic images was desirable, and for many years no method but that of hand-drawing was known. With the advent of photography these conditions were changed, and it was at once recognised that the combination of photography with the microscope had great possibilities, and opened up a fresh field for work.

Photo-micrography has become, therefore, an important branch of microscopic work, but it still suffers from certain disabilities. When a microscopist is observing an image under the microscope, he can so focus any particular plane of the object as to bring out the part he wants, and can in a drawing delineate this part only, suppressing the portions of the image not required or entirely eliminating any ill-defined areas. In viewing an object each successive plane can be focussed, and so a general idea of its structure in depth can be obtained.

The quality of penetration, or the power of showing more than one optical plane at a time, is not evident in any firstrate high-power objective: in fact, the greater its excellence, the less the depth of focus which it possesses. The photographer has difficulty in embodying these appearances in his picture, 
and this now really constitutes the chief, if not the only, objection to photography as applied to the microscope.

Photo-micrography may be approached from either of two sides : by the microscopist who wishes for a method of recording his observations, or by the photographer who has exhausted his interest in landscape work, portraiture, or what is usually referred to as ordinary photography, and is looking for a new field of work.

The microscopist will certainly have by far the easier task, as the difficulties encountered are chiefly those of the proper use and adjustment of the microscope, rather than the mastering of the technical difficulties of the purely photographic portion; even the latter are now, by the constant simplification of photographic processes, greatly minimised.

On the other hand, the photographer who wishes to enter upon a new field will find that in order to attain any degree of success he has to master the use of the microscope as an optical instrument, and also to acquire a general knowledge of the objects which he wishes to photograph.

The earliest examples of the application of photography to the microscope are coincident with the days of the daguerreotype: so that it may be said to have started from the earliest times when photography was practicable at all. At that time, however, the microscope was in a comparatively rudimentary condition-particularly so far as its optical parts were concerned. In recent years so much progress has been made in the preparation of specimens for the microscope, and in methods of staining, that photo-micrography has steadily improved in the results which it has enabled us to obtain. Also the reproduction of photographs, whether microscopic or otherwise, for the purpose of book-illustration, is now so extremely good that this method of delineation has become possible for subjects which but a very short time ago could not have been dealt with at all.

Photography itself, however, for a long period suffered from considerable disabilities, and was surrounded by technical difficulties. The great skill and experience needed to work successfully the only process then available-i.e. the so-called 'wet-plate' process-hindered any but expert photographers from attempting it. Consequently photo-micrographic work 
was only practised by the few, although it is unquestionably the fact that the photographs then produced by two or three well-known workers have seldom been equalled, and in their class have never been excelled. With the advent of the "dryplate' method all this was changed, and photo-micrography began to take up the position in microscopy that its earliest devotees expected and hoped for it.

The difficulty already mentioned, of delineating photographically an object, the details of which extend through a series of planes, is certainly a considerable one, and in the majority of cases, almost impossible of solution. It is, however, quite within the range of possibility to select the planes that give the best general appearance, and, by careful methods, which will hereafter be described, to select the focus which is most suitable for a particular object, and to exclude more or less that part of it which is useless. It is, however, a fact that in microscopical work the objects to which this objection applies are comparatively small in number; and more particularly with the present methods of section-cutting, and of staining of preparations where the sections themselves are thin, the difficulty will not appear at all.

Photo-micrography now embraces a very wide field of work. It is applied particularly in pathology, bacteriology, and histology ; but in later years its application to metallurgy and petrology has opened up a still larger field.

Its advantage over any method of drawing must be obvious, as it is impossible to get an appearance on the photographic plate, assuming of course that the work is carried out honestly and with due regard to the principles involved, other than that which actually appears in the object itself. By its use too, the factor known as ' the personal equation' is eliminated, so that in recording the results of research only a truthful rendering of objects is possible; or at least no definite variation to suit the end in view can be made, as is possible when drawings are employed. It is also obvious that many objects, either from their intricate structure or fineness of detail, cannot be successfully drawn: but by the photographic method no detail is too fine to be delineated with perfect accuracy.

It should at once be said that to acquire any considerable 
degree of dexterity in this work, both a sound knowledge of the microscope, from its optical as well as from its practical side, is essential, and in addition, the technicalities of pure photography should be thoroughly mastered.

/ The microscopist, who is anxious to start this work, cannot be too strongly advised first to acquaint himself thoroughly with the processes of ordinary photography, and for this purpose a considerable amount of work should be done with a camera both in the field, and if possible, in the photographing of coloured paintings-such objects as flowers and still-life subjects particularly being chosen with a view to correct colour-rendering in monochrome. No mere experience with a camera of the snapshot variety is of the least assistance : in fact, it is almost worse than useless, as no idea whatever is obtained of the value of the light being used, nor are the rendering of colour and the differences in tone which occur in photography ever appreciated.

The photographer on the other hand should master, with a simple microscope in preference, the ordinary technicalities of microscopic work; he will then be able the more easily to apply his photographic knowledge in recording what he sees.

The work in general does not present any extraordinary difficulty, but a thorough mastery of the details of both branches-that is, of microscopy and of photography-is essential. Unbounded patience and perseverance are necessary if the best results are to be obtained; but it is unquestioned that with the greatly improved appliances now available, the end in view can be reached sooner than was possible even a few years back. That the science will take an even higher place than it at present occupies, the writer is convinced, but it will only be as the result of persistent effort directed by those who specialise. 


\section{CHAPTER II}

\section{THE MICROSCOPE}

Probably the majority of readers of the present book will already possess a microscope, and for these it will simply be a question of adapting it in the best possible manner to the requirements of photo-micrography. Others, however, will prefer to purchase an instrument specially suited to the work; or, again, in those cases in which the study is being taken up from the beginning, it will be useful to know some of the points to be observed in the mechanical construction of a microscope, so that a suitable selection may be made.

Microscopes have not altered in general design for very many years, and up to comparatively recent times there were two well-defined types: the one generally known as the English, and the other as the Continental, model. They differ mainly in one particular - the manner in which the body part is supported. In the English models it is generally supported on a tripod foot, which has a considerable spread. The result is that the English model microscopes are usually rigid and stable whether in a vertical, inclined, or horizontal position. In the Continental models the body is supported on one metal casting, which consists of a horse-shoe foot with two vertical projections, and between these it can be swung, a pin or rod passing through and allowing of its inclination about this point as an axis.

It is, perhaps, unfortunate that of late years the English makers have-possibly as the result of competition-gone towards the Continental model, at least in the cheaper type of instruments. On the other hand, the Continental makers have appreciated that the English design, in the matter of 
steadiness at least, was preferable, and have been inclined to construct their stands with a more stable base, in some cases reproducing the English method. The consequence will probably be that some combination of the two methods will be evolved. Should it result in the elimination of the want of stability that usually obtains in stands of the Continental type, then much will have been accomplished.

In choosing a microscope for photography the following points must be considered, and indeed for any serious work are essential. The foot should rest on three points, which should be as widely separated as possible, and should be heavy : in fact, the instrument should be so built that it requires no clamping down to its support when in a horizontal position. The body-tube, containing the eye-piece and objective, should have a coarse adjustment actuated by rackwork, which should move the tube to and fro with absolute precision. There should be no evidence whatever of backlash - that is, inclination for the teeth on the wheel that engage in the rackwork to shake or fail to move the tube the moment the head of the screw is turned; while at the same time the movement should be sufficiently easy to avoid the necessity for anything like undue strain when the tube is being moved.

The rackwork should move in the direction of the optic axis only; so that if an object on the stage is observed while the rack is moved, there should be no evidence whatever of lateral displacement of the image.

The fine adjustment is of primary importance, and any want of precision in its design or construction renders an otherwise good instrument practically useless. There are many types of fine adjustment: in fact, each maker pins his faith to some particular method, but it is not within the scope of this work to go into the details of design. It should be tested by putting an object on to the stage of the microscope, and an observation should be made with an objective of fairly high power. The fine-adjustment screw should be lightly but firmly grasped on either side by the thumb and, preferably, the second finger, so that an even pressure is exerted without strain. On turning the fine-adjustment screw no evidence of movement of the object should be discernible: it should simply come in and out of focus as the screw is gently revolved. It is nowadays 
more than ever necessary for this movement to be as slow as possible consistently with convenience in working, since owing to the large aperture of the objectives now obtainable small focal changes have a much greater effect on the image than formerly.

The microscope should be provided with a draw-tube, preferably actuated by rackwork, although this is not absolutely necessary ; the length of the draw-tube will be dependent on the objectives that are selected, but it is preferable, if possible, to have sufficient length of tube so that either ' short ' or ' long' tube objectives, as they are called, can be used at will.

The stage should be large, and very firmly supported, so that no light pressure on it-such as occurs when an object is being moved about on the stage-will result in flexure and consequent displacement of the object in relation to the optical system. The stage should have a large hole in it; or, better still, an elongated slot with its opening on the side away from the observer. The opening in any case should always be sufficiently large to allow for any sub-stage illuminating apparatus that may be adopted.

A mechanical stage is of course very convenient, but by no means essential. If, however, it is adopted, it should be of the very best possible design and construction. A bad mechanical stage is worse than useless, and will only lead to trouble. It should work quite smoothly and exactly at right angles to the optical axis. If the moderate additional expense is not a consideration, then verniers for determining the exact position of any particular spot in an object, so that a record of its position may be kept, are often of great utility. Except for very highpower work where long searches for particular appearances are necessary, the writer prefers to use an instrument with a large flat stage, and without any mechanical arrangement for shifting the object ; certainly, unless a mechanical stage of the very best make can be obtained, this is the better method. A centreing stage-that is, one in which an arrangement is made for a very small movement of the stage to facilitate the exact centreing of an object-is exceedingly useful in setting up objects for photographing.

The points so far enumerated are the principal ones in the part of the instrument that lies above the stage; but the 
sub-stage apparatus and the means for adjusting it are hardly less important. Any instrument for photo-micrography must have a sub-stage condenser, and, as will be found later, the proper use of this is only second in importance to the management of the objectives themselves. The arrangement for carrying a condenser should be thoroughly stiff and solid. Usually it is supported on an arm which extends in a direction at right angles to the stage on its under side. The condenser is carried in a fitting which has freedom of movement in the direction of the optical axis and which is actuated by rackwork for exact focussing and adjustment. It, too, should be thoroughly tested to make sure that the movement is a regular one, without lateral displacement, and that the rackwork has no backlash or stiffness in action. In some stands a fine adjustment is also provided, exactly as used for focussing the objective; but the writer has never felt the necessity for this, and regards it as an unnecessary complication-at least for any but the most critical and unusual work. A good rackwork motion, used with care, will fulfil every ordinary requirement. The condenser should be that known as the 'centreing' type-that is, it should have an arrangement for altering its position in a direction at right angles to the optical axis, so as to get it in perfect alignment with the axis of the instrument. This is usually effected (compare Fig. 1) by having two screws which engage the mount carrying the optical portion of the condenser. On the side opposite to that on which the screws are situated is a spring which keeps the mount always up to its position. The inner ring carries the optical portion and is movable, by turning either or both screws, in relation to the fixed collars above and below. Underneath the condenser an iris-diaphragm is mounted so that the available aperture of the condenser system may be altered at will. In some Continental stands there is an arrangement for throwing the condenser out of the optical axis of the microscope, so that oblique illumination may be obtained, and, further, there is usually a swing-out arm carrying the condenser which enables the whole of the optical part of the sub-stage to be thrown out of action.

For metallurgical work a rotating mechanical stage will be found almost essential, as it is necessary to be able to vary 
the direction and the angle of incidence of the light when illumination of opaque objects is desired.

The following illustrations of microscopes are chosen to indicate the various types that are at present being made by the leading manufacturers in England and on the Continent. They are not selected as being necessarily the only types suitable for the purpose in view. It may be taken as certain in purchasing a microscope that the best will be found in every way the most satisfactory, although it need not be implied that an expensive stand is a necessity. There are few really bad microscopes made nowadays; even the low-priced ones, as the result of competition, have to be well finished, and, if bought from a maker of repute, will almost invariably give satisfaction.

The instrument selected will largely depend on the work that has to be done. If high power, and work of a critical nature is required, then an instrument of the very highest class is essential ; but for ordinary work there are many microscopes of moderate price available. The writer used for some thirteen years, without interruption, a microscope of the Wales type, made by Messrs. Swift \& Son (Fig. 1), and has found it in every respect satisfactory. It was first adopted because of its being so highly recommended by that pioneer of photomicrographic work, Mr. Andrew Pringle, who referred to it particularly in the earliest editions of his book on photo-micrography. As the writer has found this microscope equal to almost any requirement, he feels justified in giving some special description of it, for it will meet the needs of the majority of photo-micrographers.

The chief advantage of this form of stand is that the centre of gravity of the instrument is practically constant whatever the inclination at which it may be used. As will be seen by the illustration the inclination of the body is effected not by swinging on a central pivot, but by the movement of a semicircular portion of the body part between two jaws, and these can be clamped by a clamping-screw so that the microscope is rigid in any position. The base of the stand has a good wide spread, so that the instrument is stable and well balanced ; the fine and coarse adjustments are entirely satisfactory, and have worked with precision under the most trying circumstances. 
Messrs. Swift have recently introduced a modification of the fine adjustment, so that it can now be obtained in the same

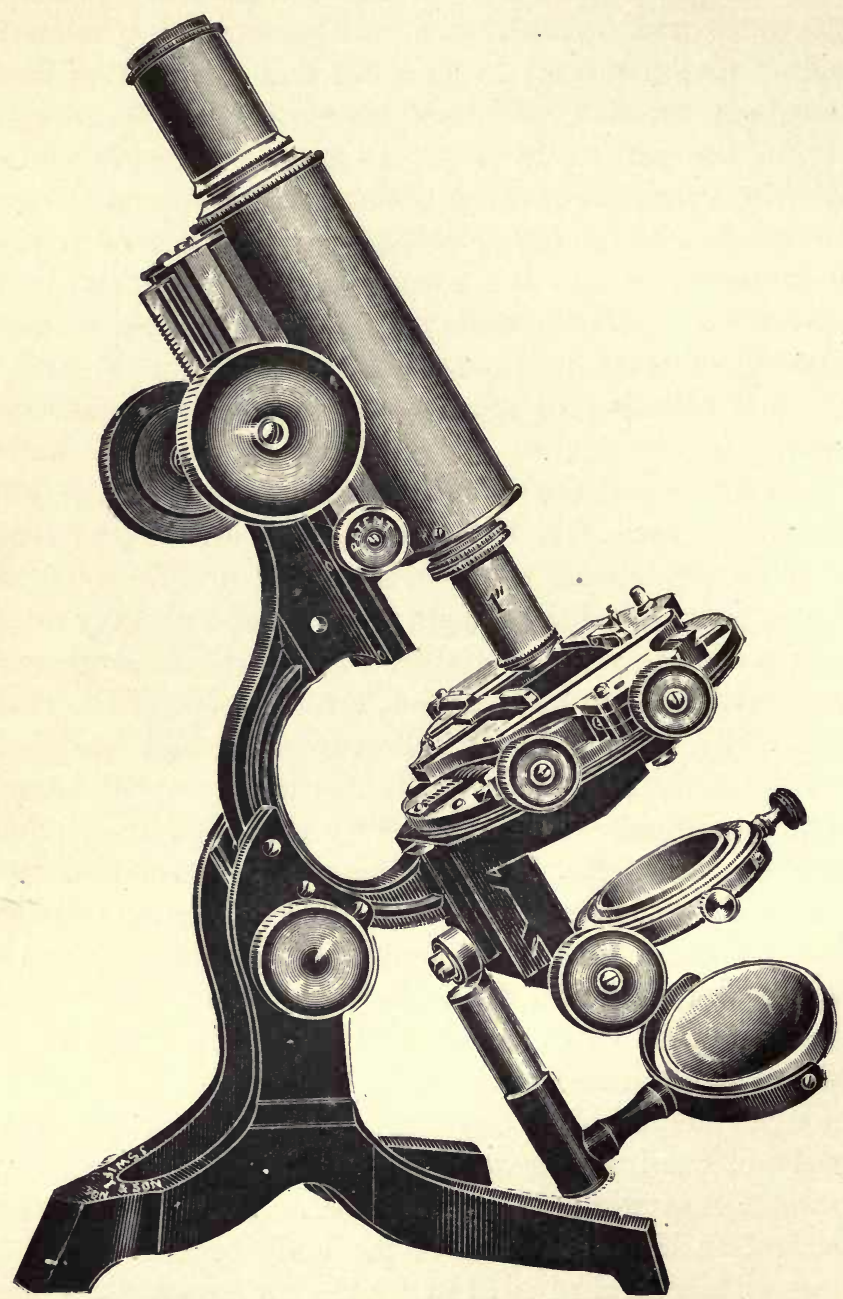

FIG. 1.-Wales type of Microscope. (Messrs. J. Swift \& Son.)

position as on Continental models-that is, in a position facing the observer when using the microscope. The fine adjustment is slow and thoroughly steady in use, and when the highest powers are being used, works with perfect steadiness. The 
stage, as shown, is of a mechanical type, and can be completely revolved; the screws for actuating it are conveniently placed, whatever the position of the stage. The sub-stage condenser has centreing-screws, with rack and pinion adjustment for focussing. The mirror is carried on a swinging arm of sufficient length to enable it to be brought well above the stage if necessary ; and this will be found of use when opaque objects are to be illuminated. The instrument has been in constant use for widely varying classes of work, and has fulfilled every requirement.

Latterly, as an instrument was required which might be available for metallurgical work and other opaque objects, as well as for ordinary work, a Zeiss photo-micrographic stand (Fig. 2) has also been adopted by the writer, and for the highest and most critical work has proved in every way suitable. It has, unfortunately, some of the disadvantages to which the Continental type of stand is subject, viz. lack of stability in the horizontal position, and somewhat cramped sub-stage arrangements; but otherwise it is an instrument of the highest merit. The fine adjustment is of very delicate construction and has perfect motion, responding to the slightest touch and being suitable for use with the highest powers used under the most exacting conditions. The body-tube is very large to minimise internal reflections and also to enable objectives of long focus and large field to be used. The special mechanical stage, designed for photo-micrographic work, has a very exact slow motion in each direction, and in the larger type has great range of movement ; it also allows of complete rotation. Either long- or short-tube objectives may be used: in fact, the instrument is designed so that it may be used for any purpose without restriction.

If the prospective purchaser of a microscope confines himself to one of the makers whose instruments are mentioned below and who are well-recognised manufacturers, he is not likely to be other than satisfied. The descriptions are practically as given in the makers' catalogues, but the writer has knowledge of, and has personally tested, each of those described.

Fig. 3 is that of an instrument well known among those using large stands, which was manufactured at the suggestion of Dr. H. van Heurck of Antwerp. The whole construction 


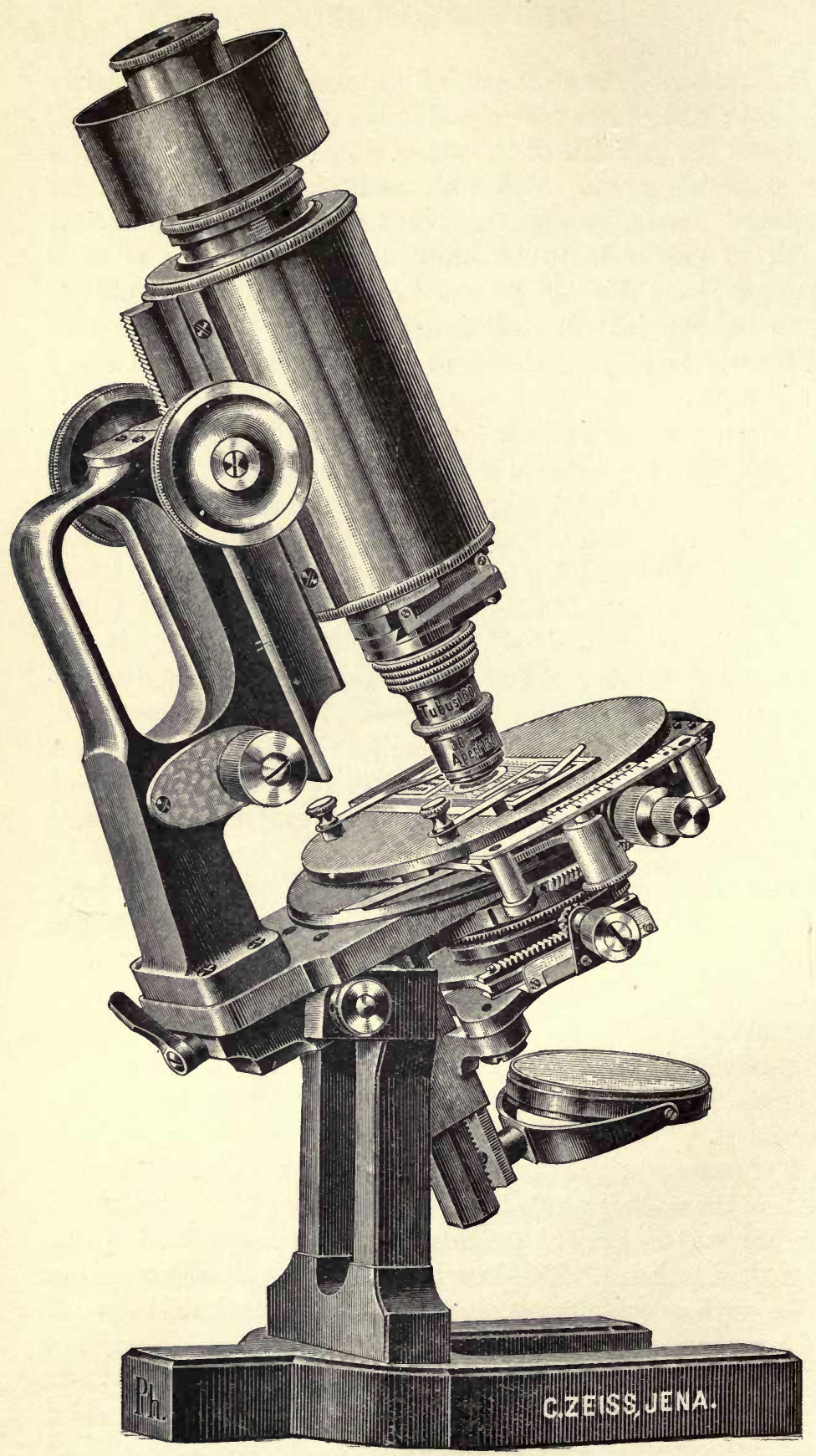

FIa. 2.-Zeiss Microscope with Photo-micrographic Stage. 


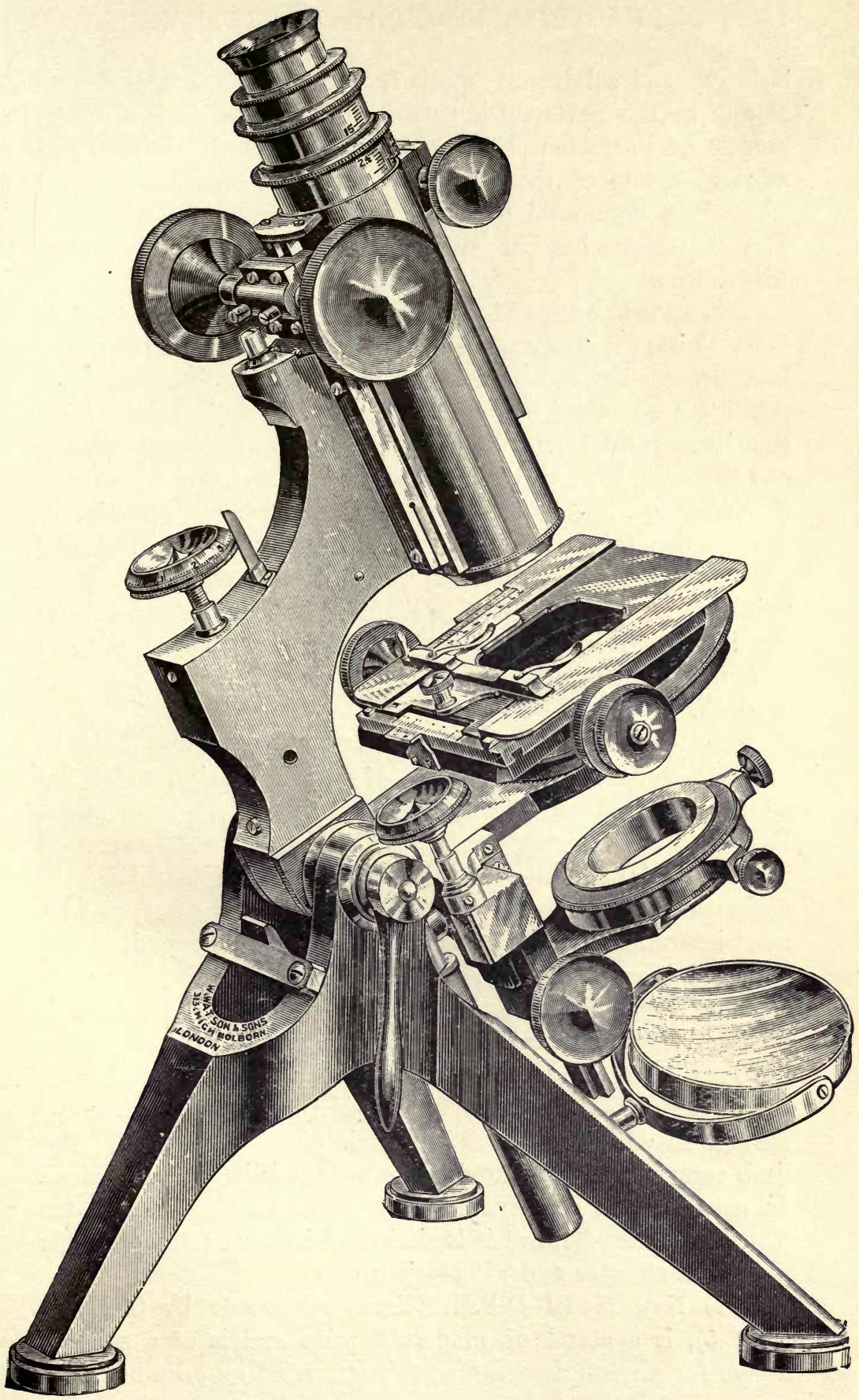

Fir. 3.-Van Heurck Microscope, (Messrs. W. Watson \& Sons, Ltd.) 
is heavy and solid, and specially designed to avoid flexure of any of the parts during work. There are three different models of it made; the illustration shows the largest and most elaborate of these. The instrument can be clamped when in a horizontal position, and is then perfectly steady. The sub-stage has in this model both coarse and fine adjustment.

Fig. 4 shows a type of stand recently introduced which, while maintaining the characteristics of most Continental models, has been provided with a foot of the English type, to give greater stability when the instrument is inclined or is in a horizontal position. It has a revolving stage, and also centreing-screws ; and there are the usual adjustments for centreing and focussing the sub-stage. The alteration of this model in the direction of the English type of foot has unfortunately not been carried out to its full extent, as the spread of the three supporting points is not quite so great as it might have been. Otherwise the instrument is a good one, is of moderate price, and will be found to answer its purpose under any ordinary conditions of work.

The Research Microscope, by J. Swift \& Son, is a well-designed and well-constructed instrument of moderate price, which has all the required adjustments to object-stage and sub-stage. Except in the design of foot it is of the Continental type. It possesses a large stage with a mechanical motion which is so designed that it has a very wide range. In common with most instruments made by English firms of this type it is well balanced in any position.

The Royal Microscope, by W. Watson \& Sons, Ltd., is distinguished by the method of attaching the stage to the other. portions of the instrument. This has been designed with the object of getting the utmost rigidity, and of avoiding any movement of the stage when high powers are used. The limb carrying the stage and the sub-stage is in one solid piece. In general it is constructed very much on the lines of the Van Heurck model, but it is simplified in certain directions to provide a cheaper and yet efficient stand.

The New Model D.P.H. Microscope, made by C. Baker (Fig. 5), is a stand of moderate price and is of a suitable design for photo-micrography. It is carried on a limb cast in 


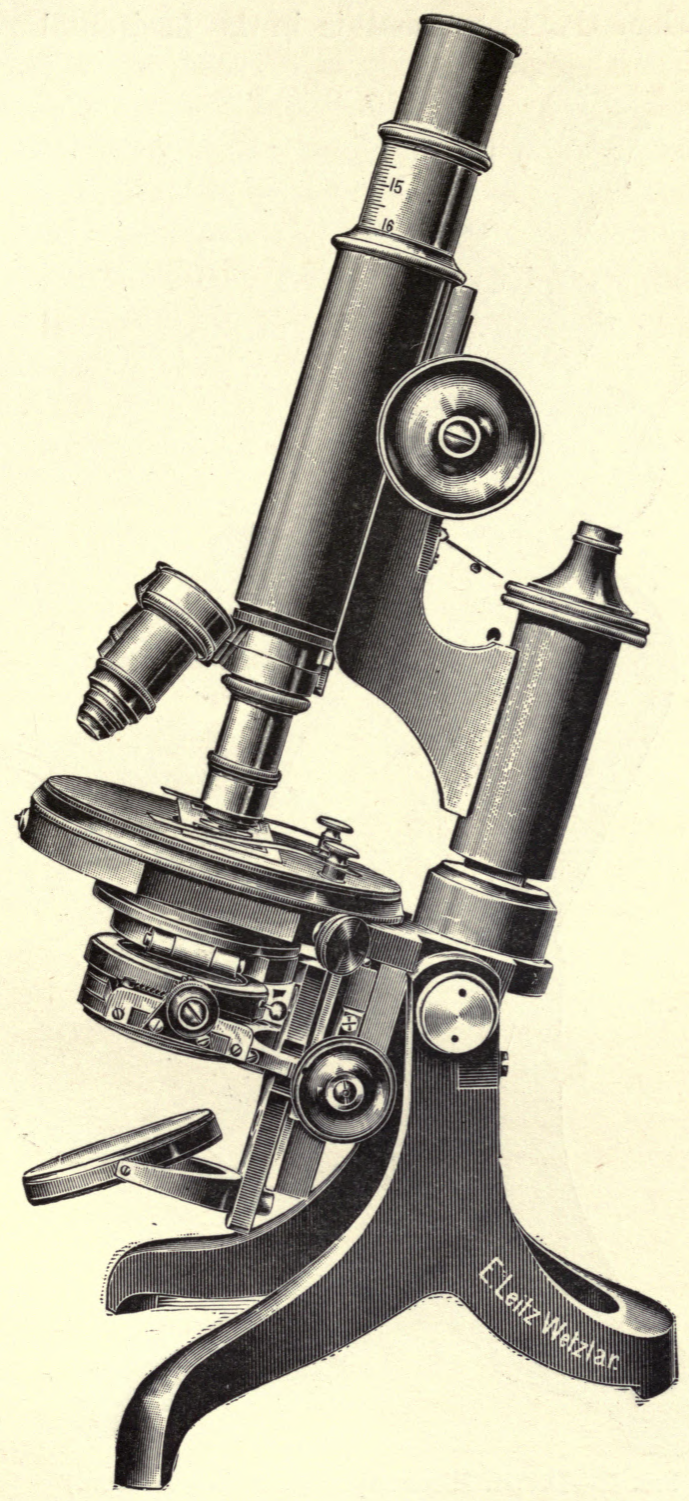

Frg. 4.-Leitz Stand, $1 a$ with English Foot. (E. Leitz, London.) 
one piece, and has a well-spread tripod foot which ensures stability when the instrument is in the horizontal position.

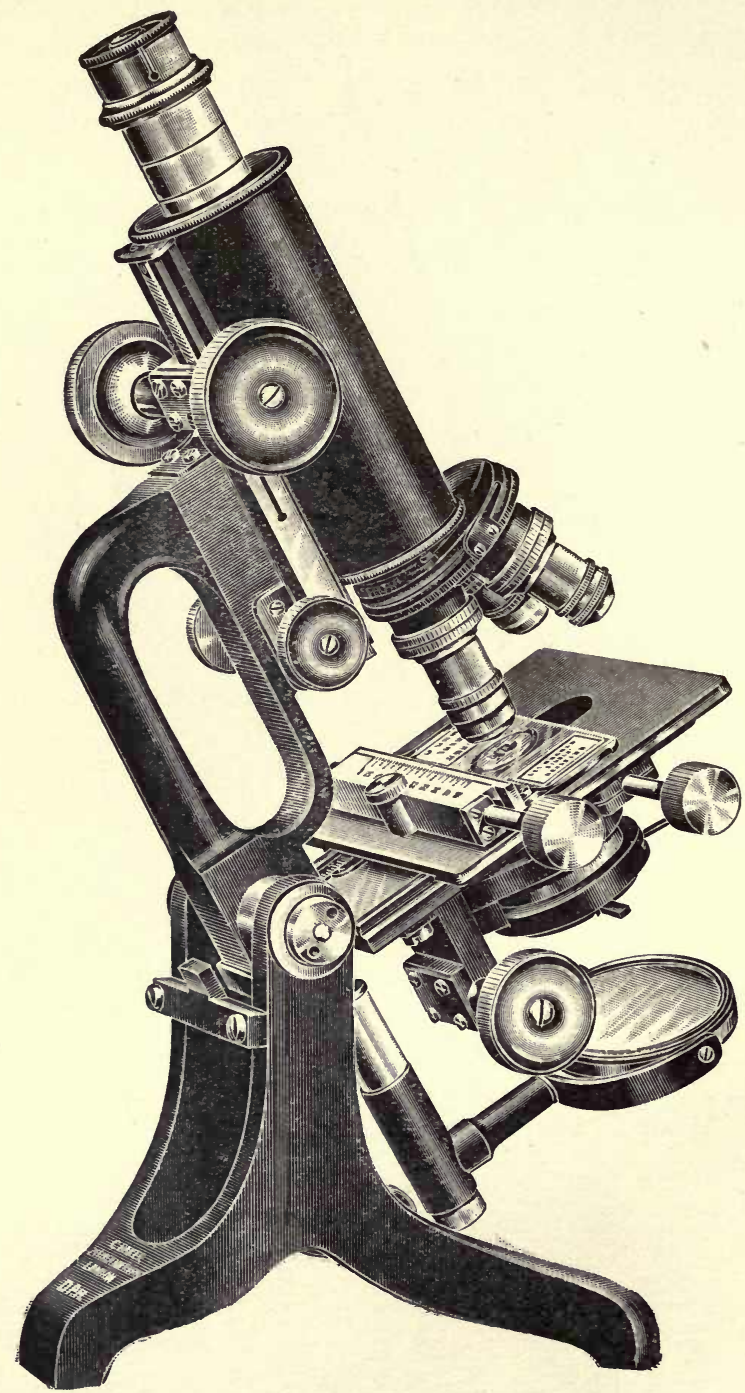

FIG. 5.-D.P.H. Microscope. (C. Baker, London.)

It can be fitted, if required, with centreing sub-stage; and a removable mechanical stage with large range of movement can also be adapted to the instrument. 
The Medium-sized Stand, H. III. (by C. Reichert, Vienna; agents, H. F. Angus \& Co., London), is an efficient type of instrument of low price which can be supplied with all necessary movements both above and below the stage. It can also be obtained with the English type of foot, thus ensuring greater stability. The handle attachment is convenient when moving the instrument after adjustments have been made, and when replacing it in position on the camera base. 


\section{CHAPTER III}

\section{THE OPTICAL EQUIPMENT : OBJECTIVES AND OCULARS}

THE most important part of the apparatus required for photomicrography is the optical system. It may conveniently be divided and treated under four headings :-

1. The objective, or magnifying system.

2. The ocular, or eye-piece.

3. The sub-stage condensing apparatus for illuminating the object.

4. The collecting or condensing lens or lenses.

As a matter of convenience the last two are separated in this manner to distinguish between the actual sub-stage condenser, as fitted to all modern microscopes, and any optical system that may intervene between the source of light and the substage condenser for the purpose of concentrating or diffusing the light. The latter optical system will be referred to throughout as the collecting-lens or lenses.

The Objective.-It does not come within the scope of this work to deal with the theory of microscopic optics, but an effort will be made to give some simple directions so that a student, or microscopist of limited experience, may be able to determine within certain limits whether the objectives he already has, or may contemplate purchasing, are satisfactory for the purpose in view. The novice must of necessity rely on the judgment of others, either depending on those from whom the apparatus is purchased, or perhaps preferably seeking the assistance of a friendly expert.

There are two well-defined types of microscope objectives now to be obtained-achromatic and apochromatic-the difference between them depending on their degree of correction for 
certain aberrations inherent in an objective. These may be for convenience again subdivided into low- and high-power lenses. The low powers are always what are known as dry lenses, and with them a magnification of not more than five hundred diameters would be obtainable. The high powers are chiefly what are known as immersion lenses; that is, between the object and the front lens of the objective an immersion fluid-sometimes water, but usually cedar-wood oil-is used; or, in the case of some lenses of very high aperture (to be obtained from Messrs. Zeiss), monobromide of naphthaline. The object of the immersion fluid is to enable a wider beam of light to be utilised by the objective. Owing to cedar-wood oil having a refractive index approximately the same as glass, the system becomes what is known as 'homogeneous'; that is, assuming the object to be mounted in contact with the cover-glass, or in a medium of nearly the same refractivity as glass, little or no refraction of the light occurs from its leaving the object, until it passes through the objective. Consequently, more light is transmitted to the objective, and a beam of wider angle can be utilised.

Fig. 6 represents diagrammatically a section through the cover-glass covering any object and the front lens of an objective. The left half represents the conditions under which an oilimmersion objective is used, and the right half shows the air-gap between cover-glass and objective which exists with dry lenses.

It will be seen that the ray $A B$ undergoes refraction at $B$ when entering the cover-glass, and it is therefore refracted towards the normal $\mathrm{BN}$, and, on leaving the glass, is again refracted in the direction $\mathrm{DA}^{\prime}$ parallel to $\mathrm{AB}$. The consequence is that the ray $\mathrm{AB}$ issues from glass into air as $\mathrm{DA}^{\prime}$, and does not enter the front lens of the objective. The ray $\mathrm{CBC}^{\prime}$ is the ray farthest from the normal that enters the lens. The condition of affairs when oil is interposed is seen on the other side, where the ray $\mathrm{EB}$ is again refracted by the cover-glass ; but, owing to the oil being of practically the same refractive index as the glass, the ray continues in the same straight line in the direction $\mathrm{BE}^{\prime}$ and enters the lens, so that it goes to take part in the formation of the image. A ray, therefore, such as $\mathrm{FB}$, which is very much more oblique and which would take the direction $\mathrm{BF}^{\prime}$ on refraction through the glass, would just enter the lens. It is evident, 
therefore, that with an oil-immersion objective more light enters, and light also of a very much wider angle, than is the case with a dry lens.

The difference between achromatic and apochromatic objectives is a fundamental one, although it must be admitted that the dividing-line is becoming ever finer. It is unfortunately the fact that some lenses described and sold as apochromatic are not exactly entitled to be so designated. Recently the term 'semi-apochromatic' has been introduced, and is now

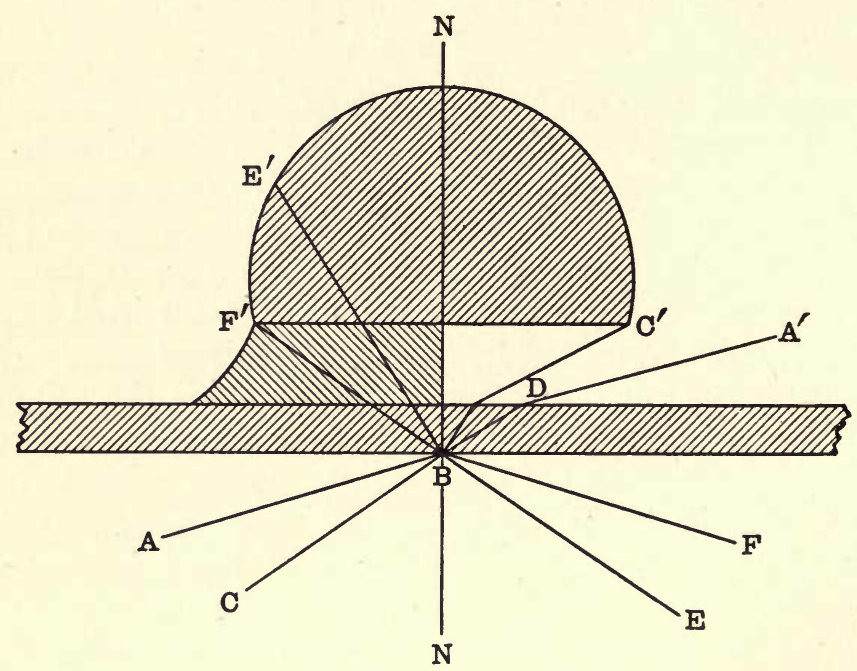

Fic. 6.-Diagram showing Effect of Oil Immersion as compared with a Dry Lens.

frequently used to describe improved achromatic lenses, specially those in which fluorite is used as one of the components of the optical system. This term would appear to be a somewhat unfortunate one, as although the lenses are an improvement on the ordinary achromatic type, yet they do not compare with the apochromatic lenses in perfection of correction. It is unquestionable that achromatic lenses have of late been greatly improved, and that their corrections are becoming ever more perfect, but they are not yet entitled to rank with the apochromats.

The difference between these two types of lenses is princi- 
pally due to the lower or higher degree of correction for chromatic and spherical aberration. In the case of achromatic lenses, the chromatic correction is only for two colours of the spectrum, whereas spherical aberration is only corrected for one colour; so that there is always an outstanding portion of the spectrum which the lens is not able to deal with properly, and which, therefore, is not utilised in the formation of the image. It becomes evident either as a more or less visible fringe of colour around the image of the object, or is diffused over the whole as a general haze with resulting loss of quality of the image.

Apochromatic lenses are chromatically corrected for three different colours of the spectrum, and for spherical aberration are corrected for two colours. For visual work, and more particularly for photographic purposes, this is, of course, a very great advantage; but, in addition, the higher numerical aperture which owing to their construction it is possible to attain in them, gives them a proportionately greater resolving power. A greater amount of light is transmitted by the objective, and consequently in photography the exposures are shortened and the resulting image is of greater sharpness and purity. Multicoloured objects-assuming of course that all portions of them are in the same object-plane-are all equally sharp and are focussed in the same optical plane. The consequence is that the image differs little in quality whether white or monochromatic light is used, and differences in colour do not entail any alteration in the focus of the objective.

A point of first importance in choosing an objective is to ascertain its numerical aperture-ordinarily referred to as its N.A. Until comparatively recently a quality known as penetration, or what is usually referred to in photographic lenses as depth of focus, was regarded as a particular advantage, and in fact was sometimes referred to as an especially good point in a lens. This is no longer the case-at least for any work of even an approximately critical nature-since the penetration of a microscope objective, so far as it exists, decreases as numerical aperture is increased. In any case penetration is a delusive quality, and it often appeared to exist when actually perfect definition was not obtainable in any plane; but as there was no absolute sharpness of the image for comparison the eye was deceived, and might even be said to imagine that such equal 
sharpness in depth did exist. This apparent depth of focus may be obtained by reducing the aperture of the sub-stage condenser, the reduction being effected by closing the sub-stage iris-diaphragm. A narrower cone of rays, therefore, enters the objective, causing it to work at a smaller numerical aperture. This is a very harmful proceeding if carried to excess, and the novice cannot be warned too strongly that such a method is not advisable for the purpose of obtaining penetration, except under very special conditions. For some classes of work lenses of small aperture are still used-particularly where it is only desired to obtain a general idea of the appearance of an object of considerable thickness ; but for critical work, whether visual or photographic, lenses of the highest obtainable aperture are to be preferred. Since all the efforts of opticians have in recent years been directed to increasing numerical aperture, this quality of penetration has been gradually reduced, until now it might be regarded as a defect rather than otherwise. In visual work some accommodation usually results from the continual raising and lowering of the objective by the fine adjustment, so that a concrete notion of the appearance of an object in depth is obtained; but in photography this is not so.

Although it has been pointed out that the quality of penetration is in general not desirable in microscope objectives, it does sometimes happen in actual practice that the delineation of structure in depth, although it may not result in a truly critical representation, is very useful. The method of obtaining this penetration by reducing the aperture of the sub-stage condenser is open to serious objections, and a very much more satisfactory way is to reduce the aperture of the objective itself. With lowpower lenses this may be effected by having immediately behind the objective, in the position where it screws into the microscope tube, an iris-diaphragm which may be contracted at will. With high-power lenses, however, this is not a practical method, as it is not possible to get a diaphragm sufficiently close to the posterior combination of the objective to stop it down effectively. Both Messrs. Leitz and Zeiss now construct what are in fact small metal funnels that drop into the back of the objective and have an opening which is nearly in contact with the back lens. These contrivances are made so that a definite reduction 
in aperture is ensured, and one of these stops has to be obtained for each aperture required. With an oil-immersion lens therefore of, say, an aperture of $1 \cdot 40$, a stop that would reduce it to 1.20 or 1.10 would probably meet all ordinary requirements. A simple diagram (Fig. 7) may explain more exactly the actual effect in increasing penetration that this stopping down of the objective really has.

Suppose the line FF to represent one plane of a thick object, and that the object may extend in depth in successive layers in the direction of the point $\mathrm{A}$. The point $\mathrm{C}$ may be, therefore, only a little below the focal plane of the objective;

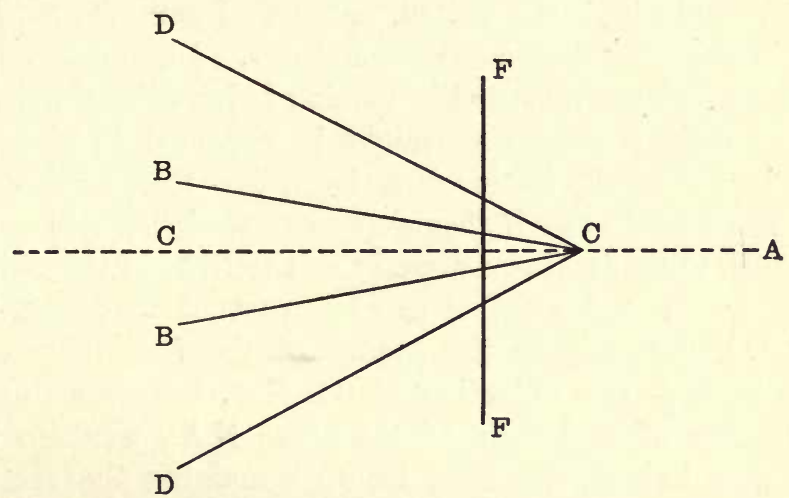

Fig. 7.-Increased Penetration due to stopping-down of Objective.

and if the illumination is by a narrow cone of light $\mathrm{BB}$, the appearance of the point, viewed in relation to the line FF, will be less spread, and approximately in better focus than if it were illuminated by a wide cone included within the angle DD. The consequence is that if there were a succession of points in the object lying just below the line FF, these would appear, with a narrow angle, less diffused than if they were viewed with a wide cone of light. It is clear also that the apparent difference between the size of the image-points in depth will be less as the angle of illumination becomes narrower. It is evident, therefore, that with a thick specimen a cone of narrow angle will give images-assuming them to be a series of points-that vary less in size than they would if the cone were wide; they will not, however, be such well-defined images, even when 
exactly in the focus of the objective, as would be obtained by utilising a higher numerical aperture.

The evil of carrying the reduction of aperture too far is that diffraction fringes very soon begin to appear around the object, and should these diffraction fringes be sufficiently evident and large enough to overlap, then a general want of definition results.

Another factor influencing penetration is the density of the medium in which the object may be mounted; a more highly refracting medium in which an object is mounted giving an appearance of somewhat greater penetration than one of less refractivity. It follows that an object mounted in Canada balsam would be better suited, on general grounds, for photographic purposes than one mounted dry. Diatoms, or similar objects, which are mounted in media of high refractive indexsuch as realgar or styrax-might be expected to give somewhat better results in this direction, other things being equal, than would be the case if they were mounted in less dense media.

Penetration decreases directly as the N.A. of the objective is increased; but what is more important in photo-micrography, it decreases as the square of the focal length. For example, a $\frac{1}{2}$-inch objective of $0.5 \mathrm{~N}$.A. has four times the penetration of a $\frac{1}{4}$-inch of the same N.A., whereas if the N.A. were halved, the focal length remaining the same, the penetration would only be halved also.

Hence, for photography, the principle is a sound one always to use an objective of the lowest power and the highest possible N.A. that will show the desired detail.

Metallurgical specimens when illuminated by reflected light are mounted without a cover-glass, and therefore without any mounting medium. This is to obviate the extra reflection that would occur from the surface of the cover-glass; and with such preparations better results are obtained by the use of oilimmersion objectives than with dry ones. The immersion fluid is in this case placed directly in contact with the specimen, and so the conditions again occur in which the greatest penetration is obtained. For the same reason in very low-power photo-micrographic work, where a magnification of only a few diameters is desired, or where even a reproduction in natural size is wanted-such as in photographing bacteriological culture-tubes 
-it is best, if such an arrangement is possible, to immerse the object in fluid : by this means the greatest possible amount of penetration is obtained.

Another quality of an objective which, particularly in lowpower lenses, is of importance, is what is known as flatness of field. With no objective is the whole field of view in proper focus at one time-at least, when a moderate-power eye-piece is used. With some oculars this does not appear to be true, but it is only due to the diaphragm in the ocular restricting the size of the visible field. It would appear that the increase of numerical aperture has tended to decrease flatness of field somewhat, as a small field is characteristic of objectives of large aperture, especially of the high-power ones. It is at times convenient to have a lens that covers a large field, even if the definition is not so absolutely critical as might otherwise be desirable. But, in general, it will be found that lenses of otherwise good quality have sufficient covering-power for any ordinary requirement. The writer is of opinion that flatness of field should not be regarded as a point of first importance; and a lens with this quality should only be selected when all other requirements have been fulfilled. It is a quality easy to determine, since with any flat object it can at once be seen where definition begins to fall off, and what proportion of the field is actually in critical focus at one time.

It may incidentally be remarked that with a good objective, although the whole field of view may not be in focus at one time, any alteration of the fine adjustment should result in successive zones of the field coming into focus and the quality of the image should be practically as good at the margin of the field as it is at its centre.

On the numerical aperture of an objective depends its power of making visible and separating fine details in an object, and it is dependent upon the size or angle of the cone of light collected by the objective from each point in the object. It must not be confused with angular aperture, or the method of indicating the aperture of photographic lenses, as these are dependent on the total angle of the field of view. Numerical aperture is purely a function of the objective, the ocular only amplifying the image, and adding nothing to the resolving power of the optical combination, 
The term ' resolving power' means the power of resolving or properly delineating fine regular structure, and must not be confused with magnifying power or any arrangement by which mere amplification is obtained. Neither does it indicate the limit of visibility; small isolated objects, or the components of irregular structure, may be seen that are considerably smaller than the distance between the components of a regular structure - such, for instance, as occurs in a diatom or a mechanically ruled grating. Consequently it is desirable to use lenses of the highest obtainable aperture, but of the longest focus-in other words, the lowest power lens that will show what is required. It is seldom necessary to master the various methods of measuring the N.A. of objectives, as this is usually marked on the lens mount. Good makers now take care that the aperture which they claim is justified; but as it may happen that such methods of measurement are required, the best known and most reliable of these are described in the Appendix. A very complete table is published with the Journal of the Royal Microscopical Society, setting out the number of lines per inch that may be resolved with a given aperture, under given conditions of illumination, and reference should be made to this if such information is required.

It may at once be stated, that where the question of cost is of no consequence, then a series of Zeiss apochromatic lenses will place the photo-micrographer in the possession of as fine a series of objectives, with the highest N.A., as can at present be obtained. On the other hand, achromatic objectives are now being improved so greatly and continually that no one need despair of producing work of a very high class by their aid, especially if care is taken, by methods to be explained later, to ensure that any outstanding colour for which they are not corrected is eliminated.

Objectives are described in terms that represent their approximate initial magnifying power, without an eye-piece, and this description does not of necessity indicate the distance which there may be between the front of the lens and the object; although there is, speaking broadly, some relation between this distance and the power of the objective.

Owing to the desire for higher aperture lenses, the indication by makers of focal length has become somewhat loose; for it is 
both optically and mechanically easier to produce a lens of high aperture and proportionately high magnifying power than the reverse. The consequence is that a lens which, for instance, might be described as a one-inch objective, with an aperture that would be a high one for such a focal length, is in some cases little more than, say, three-quarters of an inch. This is shown by Fig. 8, where $a$ was photographed with a one-sixth-inch achromatic objective, and $b$ with a 4 -mm. apochromat; it will be seen that the achromatic lens gives a distinctly higher magnification although the focal lengths are practically the same.

Apochromats are generally described with more exactness, as their manufacture was for some time confined to one firm, and consequently they have been more carefully standardised.

If it is desired to know the exact magnification of an object, either visually or photographically, it will be necessary to determine it by the aid of a micrometer. The methods to be employed for such determinations will be fully described later. (See Chap. VIII, p. 153.)

Reference should here be made to some new objectives which have been recently produced by Messrs. Zeiss, to computations by Dr. von Rohr, and termed by them 'monochromats.' These are constructed for photography only, and, as their name indicates, they are only designed for use with monochromatic light of a definite wave-length, since they are not chromatically corrected. To make them as far-reaching as possible in their optical efficiency they are specially designed for use with ultra-violet light of short wave-length. (See Chap. XI, p. 250.)

For exhaustive methods of testing and choosing objectives, the reader must refer to books treating of microscopy purely, 1 but it may be convenient if some simple tests are indicated here, so that in purchasing lenses reliance will not be entirely placed on the seller, and a serious worker will derive some extra satisfaction from discerning the differences in the lenses for himself. It is advisable, however, to purchase only from makers of high repute, and in that case no difficulty will be encountered in

1 See Carpenter's Microscope and its Revelations (J. \& A. Churchill, London) or Spitta's Microscopy (John Murray, London). 
obtaining lenses that are satisfactory. The additional cost will always be found, in the long run, to be worth incurring.

There are certain well-recognised tests for objectives, and it is often asserted as a recommendation that a lens will resolve the details of a particular test-object. This is regarded as a proof of its excellence, but it is by no means a sufficient or satisfactory method. In any case, the test-object should be one well known to the observer ; for example, in a Podura scale, or in certain diatoms, there is so much variation in the objects themselves that they often afford little information as to the quality of the objective.

But where an object is known, then an expert observer can tell by comparing the appearances with different objectives what the quality of the latter really is. For comparative purposes therefore such test-objects are of undoubted value.

For low-power objectives it is probable that no better test exists than an object illuminated by oblique light, so that it shows up bright on a dark ground-i.e. by what is ordinarily known as 'dark-ground illumination.' This condition may be obtained by putting into the sub-stage condenser a stop which cuts out the central rays and only allows the peripheral rays to pass ; these impinge on to the object at a considerable angle, so that no rays enter the objective unless they are refracted or reflected by the object. Such stops for oblique illumination are usually supplied with microscopes, and are adapted for use with the sub-stage condenser. With powers of one inch or less, this is perhaps as good a test as can be used, and with it the image should appear perfectly sharp and keen, with no haziness or evidence of diffused light at the edges. A suitable object for this test is a section of an Echinus spine, or an unstained botanical section. For tests with axial illumination, the well-known object, the blow-fly's tongue, will be found suitable; the hairs on its outer edge should show sharp and black, and the points of each hair should be seen perfectly sharp to the extremity. There should be no indication of nebulosity or duplication of the edges of the image.

With any object-glass very useful information as to its defining power can be obtained by using with it a high-power eyepiece-say, one magnifying fifteen times. With an achromatic 
objective it will be a good lens that, with any ordinary testobject, will stand this test without serious loss of definition. One in which the image remains sharp and clear with an eyepiece magnifying twelve times is, speaking broadly, a good lens.

With apochromatic objectives this does not apply, for in low and moderate powers these will not break down or show any evidence of deterioration of the image if an eye-piece is used of the highest available power-namely, twenty-seven times.

With dry lenses of less focal length than one inch, the Podura scale, or any of the coarser diatoms, will answer for purposes of testing. In any case the scale or diatom must be selected so that it has well-defined markings, and these should always show a clean bright image, with no nebulosity or haziness at the edge. What are known as the "exclamation marks' on the Podura scale should show up sharply, with their extremities going off to a sharp point.

For oil-immersion objectives, one of the finer diatomssuch as Pleurosigma angulatum, or Amphipleura pellucida-is the recognised test; and certainly for objectives of high aperture either may, in the hands of an expert, fulfil every requirement. If, however, such diatoms are employed, the differences in appearance with varying methods of illumination are so marked that it is impossible for anyone not an experienced microscopist to infer anything from them. The writer is of the opinion that for an ordinary examination of an oil-immersion objective, a stained preparation of bacteria is to be preferred. With this, in conjunction with the method already mentioned for testing the effect of high-power oculars, a great deal can soon be learnt of the qualities of an objective. Preparations should be chosen that are sharply stained, and it is advisable to select one such as the Bacillus tuberculosis, which has a segmented or beaded appearance; or some sporing organism-such as Bacillus filamentosus-since in these it is easier to observe the more subtle differences in the quality of the image.

For testing for chromatic and spherical correction, as well as for flatness of field, there is no better object with either high or low powers than the Abbe test-plate. This, further, has the advantage that where thickness of cover-glass is of great importance (as it unquestionably is with any high-power 
dry lenses), the most perfect correction that corresponds to a particular thickness can be estimated with exactness. The test-plate consists of six discs of thin microscopic coverglass of accurately determined thickness $(0.09 \mathrm{~mm}$. to $0.24 \mathrm{~mm}$.) cemented side by side. Each of these discs is silvered on its lower surface, and has a series of lines ruled on it, so that it is in effect a coarse grating of alternating silvered lines and clear spaces. The lines are usually not quite opaque, but transmit sufficient light to enable the grain of the silver deposit to be seen. On examination under the microscope with a low power, therefore, a large number of these lines come into the field, but with high powers possibly only two or three lines will be shown: in either case the object is so thin and each line has such a number of prominences on either edge that it affords an extremely valuable, and comparatively easy, method of at once determining some of the qualities of the objective under examination.

Under axial illumination and when the image is exactly in focus the edges of the lines should appear perfectly sharp with no apparent doubling; neither should there be any but the very slightest indication of colour fringes with achromatic lenses.

The critical test is to use oblique light, and the light should fall in a direction at right angles to that of the lines ruled on the test-plate. Each objective should be tested first by oblique, and then by direct axial light, and on changing from one method to the other no alteration in the focus of the objective should be necessary - that is, the object having been focussed by oblique light, its image should appear to be equally well defined when illuminated by axial light. This test indicates the degree of correction of the lens for spherical aberration.

To test for chromatic correction the same methods of illumination should be arranged for. An achromatic lens should show only narrow fringes of colour on each side of the lines on the test-plate, and these should be the complementary colours of the secondary spectrum, on one side greenish, and on the other a reddish violet. With apochromatic objectives of the highest quality-particularly those made by Messrs. Zeiss - there is little or no evidence of any coloured margin to the lines under any conditions of illumination.

In working with a test-plate the light used should be fairly 
bright and as white as possible. It is also necessary to have one or two oculars of high power.

It is probably understood that the value of an objective

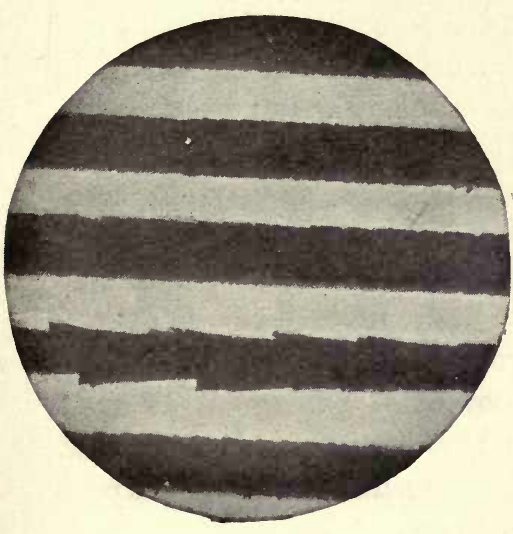

a

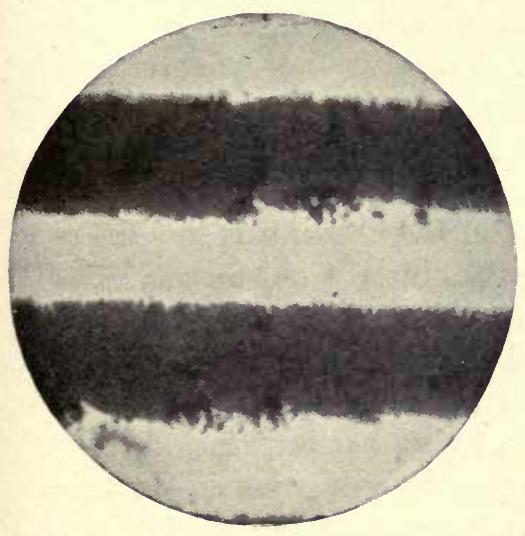

C

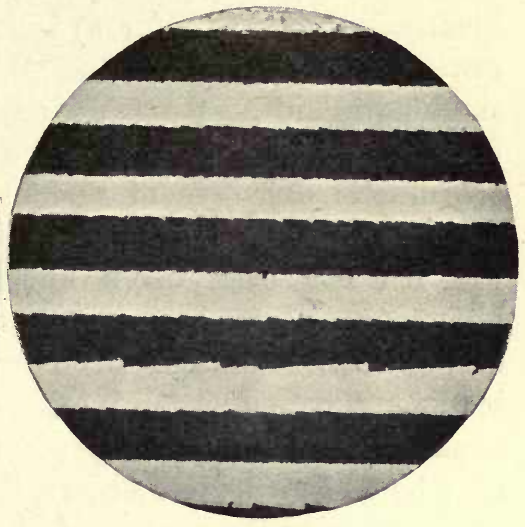

b

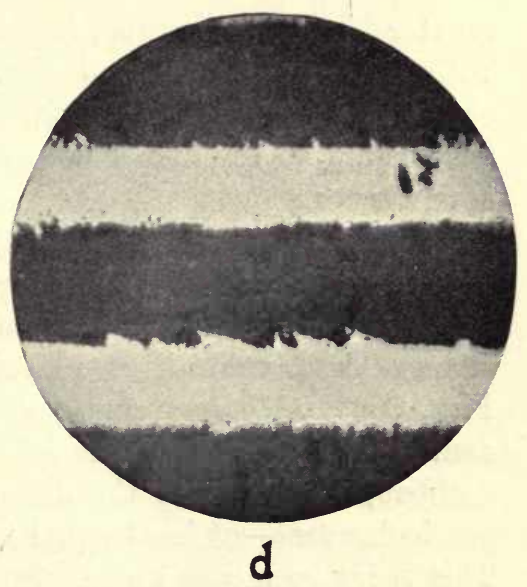

Fic. 8.-Photographs of Abbe Test-plate.

depends not on its magnifying power, but on its ability to resolve fine details, and this is purely a function of its aperture. In testing objectives for ordinary work, it is not only necessary to determine the resolving power, but more especially to find out the quality of the image, and the power which the objective 
has of forming an image. The tests already mentioned, while in no way entitled to be regarded as exhaustive, may assist an ordinary observer in gaining some information as to the extent to which his objectives have this property. The illustrations (Fig. 8, $a, b, c, d$ ) will give some idea of the appearances that may be expected when using certain objectives in combination with a particular ocular. They are all photographs of the Abbe test-plate. Fig. $8 a$ is taken with a one-sixth-inch achromatic objective of undoubtedly good quality, in combination with a No. 2 projection ocular. For comparison, Fig. $8 b$ has been taken under exactly similar conditions, but a 4-mm. apochromatic has been used instead of the one-sixth-inch achromatic. The difference between them both in definition and general brilliancy of the image is at once apparent, and will give a general idea of the appearance that may be expected when observations are made for the purpose of determining the quality of objectives.

Figs. $8 c$ and $8 d$ are taken under similar conditions, except that projection-ocular 4 , in conjunction with a somewhat greater camera extension, has been used. Fig. $8 c$ is taken with the achromatic, and Fig. $8 d$ with the apochromatic objective. Here again the difference can be seen immediately, and owing to the higher power ocular employed is decidedly accentuated.

Another point worthy of note in these figures is the difference in centration that occurs with different objectives. The two objectives were screwed into the same sliding objectivechanger and were then placed on the microscope: when Figs. $8 a$ and $b$ and also $c$ and $d$ are compared, it will be seen that a totally different field of view has been included by each objective. Thus the difference in centration is-in this particular case-at least equal to the area of the"whole field. This is by no means an exaggerated instance, and is a state of affairs that may be expected when any considerable number of objectives are being used in succession. It demonstrates clearly the necessity of using a method of changing the objectives which will admit of their correct centration to on $\theta$ another and to the optical axis of the microscope.

A good selection of objectives for photo-micrographic work would be the following :- 
1. A Planar or micro-Summar, or a lens of similar construction (preferably one of rather long focus, either the 75-mm. or $100-\mathrm{mm}$.), for photographing large objects or sections.

2. A $1 \frac{1}{2}$ - or 1-inch achromatic, or $35-\mathrm{mm}$. apochromat.

3. A two-thirds-inch or 16-mm. apochromat.

4. A one-third-inch or 8-mm. apochromat.

5. A one-sixth-inch or 4-mm. apochromat with adjustment collar allowing of correction for thickness of cover-glass (Fig. 9).

6. A one-twelfth-inch oil-immersion achromatic, and either a 3 -mm. or 2 -mm. (N.A. $1 \cdot 30$ or $1 \cdot 40$ ) apochromat. The $3-\mathrm{mm}$. apochromat of Zeiss should be chosen in preference to a $2-\mathrm{mm}$. for general work. Its greater focal length gives it a longer working distance, and it may be used with the highest power oculars.

The possessor of either series of these lenses would have a complete battery, and would be in a position to deal with any class of photo - micrographic work. The apochromats should be chosen if possible; but, as

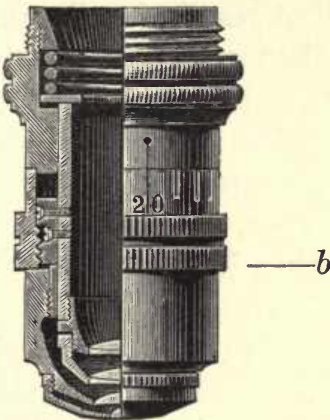

FIG. 9.-Apochromatic Objective fitted with Correction-collar, b. (Zeiss.) already stated, it need not bo regarded as an insuperable obstacle to achieving fine results if these cannot be obtained. (It should be noted that the $35-\mathrm{mm}$. and $70-\mathrm{mm}$. apochromatic objectives are made for direct projection or photography only, and should not be used in conjunction with an ocular.)

In cases where such a complete outfit as the above cannot be procured, then a one-inch and a one-sixth-inch lens will probably be most useful for general work. If bacteriological work is to be attempted, but little can be done without the addition of a one-twelfth-inch oil-immersion objective; and where it is intended only to do the latter class of work, then an oil-immersion objective, with a two-thirds-inch in addition, could be made to answer all ordinary requirements. It would certainly be better, if possible, to acquire, say, the $16-\mathrm{mm}$. and the $3-\mathrm{mm}$. apochromats, as with these two lenses, 
and with suitable combinations of ocular and camera extension, almost any degree of magnification could be obtained, except of course if large objects are to be photographed. For the latter any good-quality photographic lens-preferably an anastigmat-might be utilised.

The initial magnification obtainable with various typical objectives, and also the same lenses in conjunction with certain specified oculars, are indicated in Tables I and II.

It must be understood that these magnifications only apply when exactly the conditions indicated are fulfilled. They will, however, sufficiently indicate the magnifications that result visually, and are obtained in the position of the eye when using the ocular indicated. The term 'high power,' as applied to an ocular, is a relative one. In visual work, an ocular magnifying eight times is of medium power; but in photography it is usually regarded as of somewhat high power, since the distance the image is projected has to be taken into consideration.

Visually, an image is observed immediately above the ocular; but the projected image is seen at some distance away, with the result that greater amplification is obtained, the degree of amplification depending on the camera-length. The difference between the visual and projected image must, therefore, be borne in mind when speaking of the relative effect of different oculars.

The magnification resulting from the combination of any of these objectives with any compensating ocular is obtained by multiplying the initial magnification by the number of the ocular.

With most microscopes an appliance, called a nose-piece, for rapidly and easily changing the objectives, is now supplied. They may be obtained either double, triple, or quadruple, carrying two, three, or four objectives respectively. They are made so that the objectives they carry are screwed in, the nose-piece itself being screwed into position on the microscope where the objective usually goes. This enables the objectives to be successively brought into the optical axis, as required, with rapidity and ease. While they are useful for visual work, they are rarely to be recommended for photographic purposes; since it is unusual for a nose-piece to be so accurately 


\section{Table I}

MAGNIFICATIONS WITH ACHROMATIC OBJECTIVES AND OCULARS DESIGNATED BY LETTERS

English Tube-length $=10$ inches.

\begin{tabular}{|c|c|c|c|c|c|c|c|}
\hline \multicolumn{2}{|l|}{ Nominal Focus. } & \multicolumn{6}{|c|}{ Magnifying Powers with the Various Eye-pieces. } \\
\hline & & A & B & C & $\mathbf{D}$ & $\mathbf{E}$ & $\mathbf{F}$ \\
\hline 1 & 1 & 60 & 90 & 120 & 150 & 180 & 210 \\
\hline & $\frac{1}{2}$ & 120 & 180 & 240 & 300 & 360 & 420 \\
\hline & $\frac{1}{4}$ & 240 & 360 & 480 & 600 & 720 & 840 \\
\hline & $\frac{1}{6}$ & 360 & 540 & 720 & 900 & 1080 & 1260 \\
\hline & $\frac{1}{8}$ & 480 & 720 & 960 & 1200 & 1440 & 1680 \\
\hline $\begin{array}{c}\text { Homogeneous } \\
\text { immersion }\end{array}$ & $\frac{1}{12}$ & 720 & 1080 & 1440 & 1800 & 2160 & 2520 \\
\hline
\end{tabular}

\section{TABLE II}

MAGNIFICATIONS WITH APOCHROMATIC OBJECTIVES

Tube-length $=160$ millimetres.

\begin{tabular}{|c|c|c|c|}
\hline & $\begin{array}{c}\text { Equivalent Focus. } \\
\mathrm{mm} \text {. }\end{array}$ & $\begin{array}{l}\text { Numerical } \\
\text { Aperture. }\end{array}$ & $\begin{array}{c}\text { Initial } \\
\text { Magnification. }\end{array}$ \\
\hline Dry series $\quad . \quad \cdot$ & $\left\{\begin{array}{r}16 \\
8 \\
4\end{array}\right.$ & $\begin{array}{l}0.30 \\
0.65 \\
0.95\end{array}$ & $\begin{array}{l}15 \cdot 5 \\
31 \\
62\end{array}$ \\
\hline Water-immersions . & $2 \cdot 5$ & $1 \cdot 25$ & 100 \\
\hline $\begin{array}{l}\text { Homogeneous - immer- } \\
\text { sions. } . .\end{array}$ & $\begin{array}{l}3 \\
2 \\
1 \cdot 5\end{array}$ & $\begin{array}{c}1 \cdot 30 \text { or } 1 \cdot 40 \\
1 \cdot 30 \text { or } 1 \cdot 40 \\
1 \cdot 30\end{array}$ & $\begin{array}{r}83 \\
125 \\
166\end{array}$ \\
\hline
\end{tabular}


constructed that the objectives it carries would, when brought into the position of the optical axis, be always equally well centred. The consequence is, as already mentioned, that if the object is centred with one objective, say a low-power one, and then a high power is brought into use, the object is not in the centre of the field, and in some cases the want of centration is so great that the image may actually be thrown out of the field altogether.

Some makers are giving greater attention to these appliances, and the results obtained are better than formerly; but it is still rare for a triple or quadruple nose-piece to be so accurately made that all the objectives which it carries are equally well centred. Messrs. J. Swift \& Son have, however, recently produced some nose-pieces that are extremely accurately made and-at least, when they leave their hands-admit of little improvement in this respect.

Another objection to these multiple-changers is that a considerable additional weight-consisting of two, three, or four objectives as well as the changer itself-is thrown on to the bodytube of the microscope ; and as this weight is thrown only on one side it is a very real objection in critical work. A further disadvantage is that it is very difficult to ensure that the optical axis of the objectives are in exact alignment with the optical axis of the microscope, and should this occur with a lens of large aperture it would seriously interfere with its performance. It is far better, if possible, for photo-micrographic work to do without such a method of changing, as though it may be convenient there is too great a possibility of introducing errors in the resulting photograph.

An arrangement that is not open to any of these objections is the sliding objective-changer made by Messrs. Zeiss (Fig. 10). In these a slide is screwed on to the microscope in the position usually occupied by the objective, and it is so arranged that an objective may be inserted from any direction. This slide becomes a permanent attachment to the microscope body-tube. On each objective a counterpart of this is screwed, so that it is only necessary to slide one portion of the changer into the other to change any objective. There is an arrangement on each changer for centreing the objective accurately to the optic axis of the microscope, and this adjustment should 
be effected when the objective-changers are attached to the lenses with which they are to be used. Having once been properly centred and adjusted, they should not be taken off their respective objectives, but should always be maintained in position. The objectives will then always return to their proper position exactly in line with the optical axis of the instrument. These appliances are so arranged that when
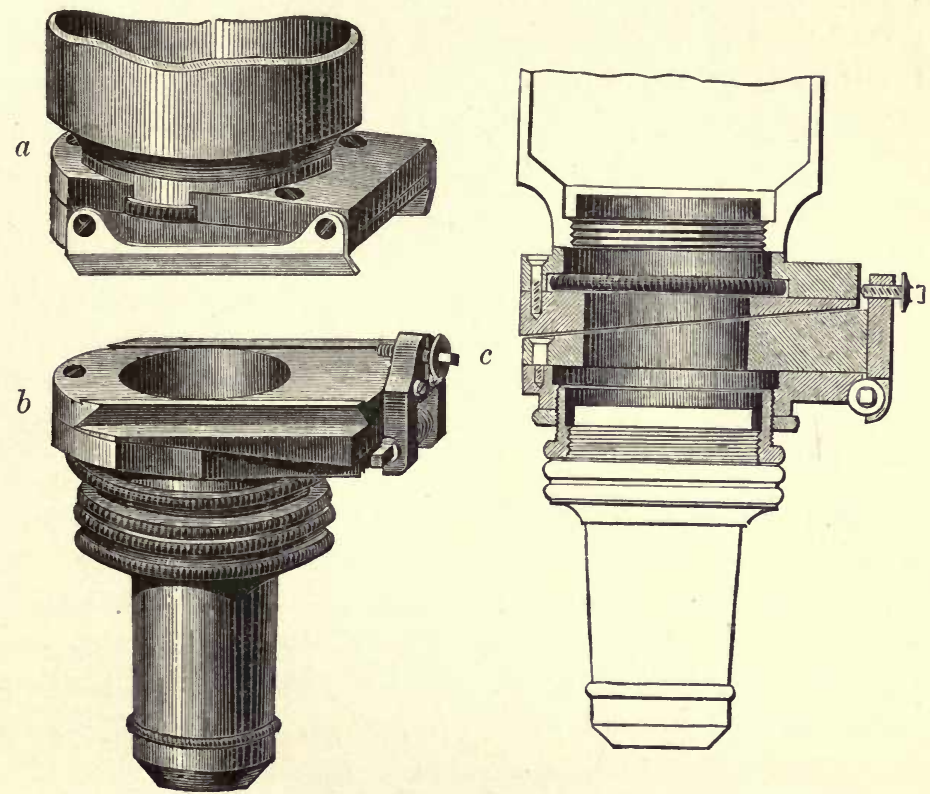

FIG. 10.-Sliding Objective-changer. (Zeiss.)

$a$, tube-slide ; $b$, objective-slide with objective attached ; $c$, intersection. (Full size.)

sliding out a lens the direction of motion is upwards, and therefore away from the object on the stage; there is therefore little likelihood of damaging the object on the microscope stage if the objective is changed, without racking up the body-tube.

It should be carefully noted when using either the nose-piece or objective-changers that due allowance must be made for these in estimating the tube-length. (See Chap. VII, p. 151.)

A simple objective-changer which is not open to the objections urged against those of the double or triple nose-piece type is 
that made by Messrs. W. Watson \& Sons. The advantages put forward by the makers are that it is very thin and only increases the tube-length by $12.5 \mathrm{~mm}$.; that it is simple and reliable in use, and as it is made of magnalium it is very light, and so adds but little to the weight to be carried by the microscope tube. The turning of the milled head results in the inversion downwards of a pair of jaws having a screw-thread cut upon them. The objective is placed in the aperture, and directly the milled head is released, the jaws are carried back and engage the threads of the objective. It therefore acts as a permanent attachment to the body-tube of the microscope, and each objective may be inserted without the necessity of screwing it in, as is the case when some such arrangement is not in use. Of course it does not admit of exact centration of each objective as does the Zeiss changer, but in use it would certainly be found to be more exact than a multiple nose-piece. With moderate- or low-power objectives this would not be such a great objection, as small errors of centration are not so apparent.

The Ocular or Eye-piece.-An important optical part, and one which in the case of the apochromatic objectives is an essential portion of the magnifying system, is the ocular. Its function is to pick up and transmit to the eye-or, in the case of projection-oculars, to the photographic plate-the image already formed by the objective, always amplifying it to a greater or less extent.

There are many types of oculars, but for microscopic work the two almost universally used are those known as the Huyghenian and the Ramsden. These may again be divided into ordinary and compensating : the latter distinction depending on whether they are for use with achromatic or apochromatic objectives. Both of the first-mentioned types occur in the Zeiss series of compensating oculars - the lower power oculars being of the Huyghenian and the higher ones of the Ramsden type.

In referring to the ordinary oculars, which are suitable for use with achromatic objectives, some makers have adopted an arbitrary method of description-simply designating them by a letter, which gives no indication of their amplifying power, although custom has decided approximately what each letter may mean. They are made in a series of regularly increasing 
power, and are called by the letters A, B, C, D, E and F, the magnification usually being $6,9,12,18,21$, and 24 .

The images formed by achromatic objectives are in them-

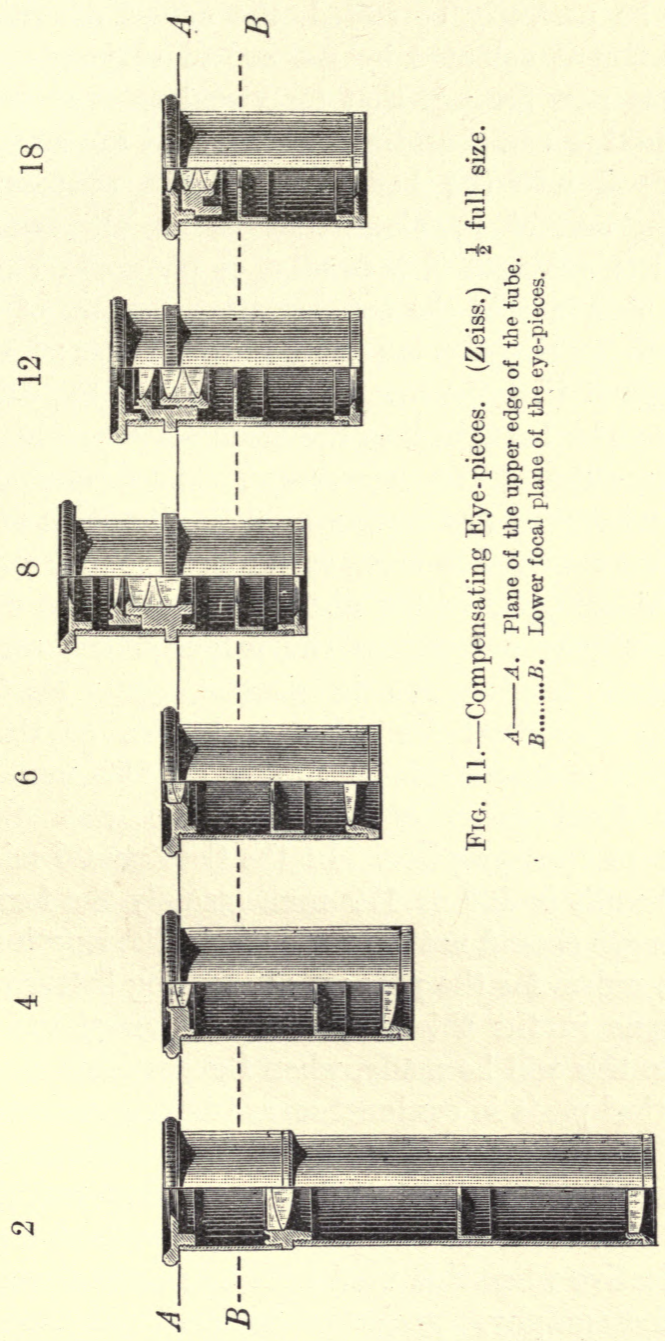

selves complete, and the ocular should cause no subsequent alteration in their optical quality. In the case of the apochromatic objectives this is not so. When these lenses 
were first designed by Professor Abbe, it was found convenient in the low powers to introduce a chromatic error which the ocular could correct. Consequently, if an apochromatic objective is used with an ordinary ocular it does not appear to be perfectly corrected, the actual condition being that the different coloured images are of varying dimensions. Most makers now arrange that for visual work their series of oculars, whether compensating or otherwise, are so made that when dropped into the body-tube of the microscope they are in their correct position-that is, no alteration of the mechanical tube-length is necessary, as shown in Fig. 11. It is for this reason that the compensating oculars of Zeiss are made in both the Huyghenian and Ramsden types ; for it is only by this method that the lower focal planes of the whole series of oculars can be brought into the same position when inserted in the draw-tube of the microscope. Also, when a change of eye-piece becomes necessary very little alteration of the fineadjustment is required to bring each objective into exact focus.

The 'tube-length,' or the distance between the ocular and objective, in part determines the magnifying power of the system. Two lengths are in general use. Most English microscopes are fitted for what is known as the 'long' tube, with a distance of 10 inches or $250 \mathrm{~mm}$. between the upper focal point of the objective and the lower focal point of the eye-piece. In the Continental microscopes this tube-length is 160 or $170 \mathrm{~mm}$. (usually the former), and the tube-length should not be varied from the length stated by the maker, unless for the purpose of securing better correction for variations in the thickness of the cover-glass. Further reference to this will be made, when the methods of using the various optical parts in conjunction are described, in Chap. VII, p. 154. In the case of compensating oculars these are distinguished by numbers, and the number represents the number of times that the eye-piece magnifies the image formed by the objective when it is used with its standard tube-length.

For photography, projection-oculars will be required when apochromatic objectives are used; with achromatic objectives they are also advisable under certain conditions. If an ocular is used at all, they are in fact the only reliable ones. In these the same principle is adopted as in the case of the 
compensating oculars, except that they are of low power, only amplifying the image from two to six times, and are constructed to project an image to a considerable distance. No ordinary ocular is designed to do this, although in fact it often happens that it will do so without any serious loss of definition. Projection-oculars do not differ from the ordinary eye-piece in the way in which they fit into the tube of the microscope, and as already stated in reference to compensating oculars they are designated by a number which indicates their magnifying power. A projection-ocular, No. 2-which to obtain this amplification should be used on a short tube-will become approximately a No. 3 when used on the long tube, and no difference in the quality of the image is appreciable when used under either condition. In each ocular is a diaphragm which is adjustable in position within the ocular itself by a spiral movement (Fig. 12). By turning a milled head at the top of the ocular the image of this diaphragm can be exactly focussed on the groundglass focussing-screen of the camera, and this operation must be carefully performed with each alteration of camera-length.

The writer is of the opinion that where ordinary achromatic objectives are used-at least those of the older types-better results are often obtained by not using an ocular at all for photographic work. The great disadvantage of this method is that a much longer camera may be required to obtain a given magnification; since it is evident that no increase in the magnification occurs, such as would of course result from using an ocular. It becomes necessary, therefore, to have a camera which is capable of extension to as much as four or five feet in length; but where this is not an insuperable objection, and in cases where apochromatic objectives are not available, it is often a satisfactory method.

It must not be overlooked that with any ordinary objective, which has an appreciable outstanding aberration of any description, an ocular only tends to increase the imperfection of the resulting image. It is evident that an ocular magnifying, say, ten times, would render much more apparent any errors in the objective than would a lower power one, and in any case it is therefore advisable in photographing to use oculars of low power, the necessary further amplification of the image being obtained by camera extension. 
With achromatic objectives of recent construction-e.g. with those which are sometimes designated as semi-apochromaticcompensating oculars work extremely well. In many of these lenses an approximation to the correction of the apochromatic lenses is obtained, and they are to some extent dependent on the oculars for obtaining the best results. Two firms have introduced a type of ocular which may be used with either achromatic or apochromatic objectives. These are called by Messrs. J. Swift \& Son the 'Universal,' and by Messrs. W. Watson \& Sons the 'Holoscopic,' eye-pieces. While they are of considerable use for visual work where a mixed series of objectives is in use, they cannot be recommended for photographic work of a critical nature. The alteration of correction is effected by altering the distance between the eye-lens and field-lens of the ocular; but at best they can only claim to be a compromise between the two recognised forms of ocular already described. A rough method of determining at sight whether an ocular is a compensating or an ordinary one, is to hold it up to the light-preferably to the sky: the image of the diaphragm then seen by direct observation will be fringed with a double band of red and orange; or, with the very low-power oculars, a yellowish edge only may be apparent. With ordinary oculars the same observation will usually disclose a well-defined blue fringe. Sometimes, to make this colour-fringe visible, the angle of incidence of the light in relation to the ocular may have to be varied, but usually it is at once apparent.

A point of considerable importance in visual work is the most suitable combination of objective and ocular. In photographic work this question does not arise, perhaps, to such an extent, as the oculars used are usually of lower power; but even then, under certain conditions, it is of importance. It is an obviously simple method for obtaining increased amplification of the image to use a higher power ocular. Such a proceeding, however, if carried to excess, introduces many difficulties. In visual work the beam entering the eye with a high-power ocular is very much narrower than is the case with a low one, and consequently any small particles of dust lying within the ocular itself or the moving specks in the fluid of the eye become painfully visible. In photographing, these specks are most 
unsightly, and, as they usually have a broad diffraction-fringe round them, become exaggerated in appearance. On the other hand, a discreet use of the method of increasing magnification by using a high-power eye-piece may be at times of great service. An example of this will perhaps best indicate the point in view.

If an ordinary one-sixth objective, with an aperture of 0.75 , is being used with a No. 8 ocular, this is probably about the highest eye-piece that such a combination permits without appreciable loss of definition. If for this is substituted an 8-mm. apochromatic objective, which has approximately double the focal length of the other lens, and which has an aperture of at least $0 \cdot 65$, it may be used with an ocular of more than double the power without suffering in any way, and there would not be a substantial difference in resolution between the two arrangements. But there would be a very appreciable advantage when using the longer focus lens ; considerably more depth of focus would be obtained, and the correction of the image in general would be of a very much higher order. It must not be overlooked that nearly all one-sixth-inch ordinary objectives are actually of shorter focus than that-so that their actual aperture is, in reality, lower relatively than it appears to be.

This question of the best combination of objective and ocular is one that must be largely determined by the conditions under which the work is being carried out, and would be influenced by the class of object that is being dealt with.

Mention must be made of an ingenious suggestion of Mr. J. W. Gordon, ${ }^{1}$ by which the narrow beams of light transmitted by high-power eye-pieces are so altered that the beam becomes a wide one. Further, by his method the black specks, due to dust or any imperfection of any description in the apparatus, or those due to imperfections of the fluid of the eye, are not apparent. A description of this method is here included; yet it must be said that the great benefit of it, from the photographic point of view, is not admitted by some good authorities. Many of the appearances which he obtains and which he claims to be improvements in the images, may in fact be due to other causes.

1 Journal of the Royal Microscopical Society, February 1907. 
Mr. Gordion's method consists essentially of a ground-glass screen, which is placed in the image plane and below the eye-piece: the narrow beam of light is thereby dispersed in all
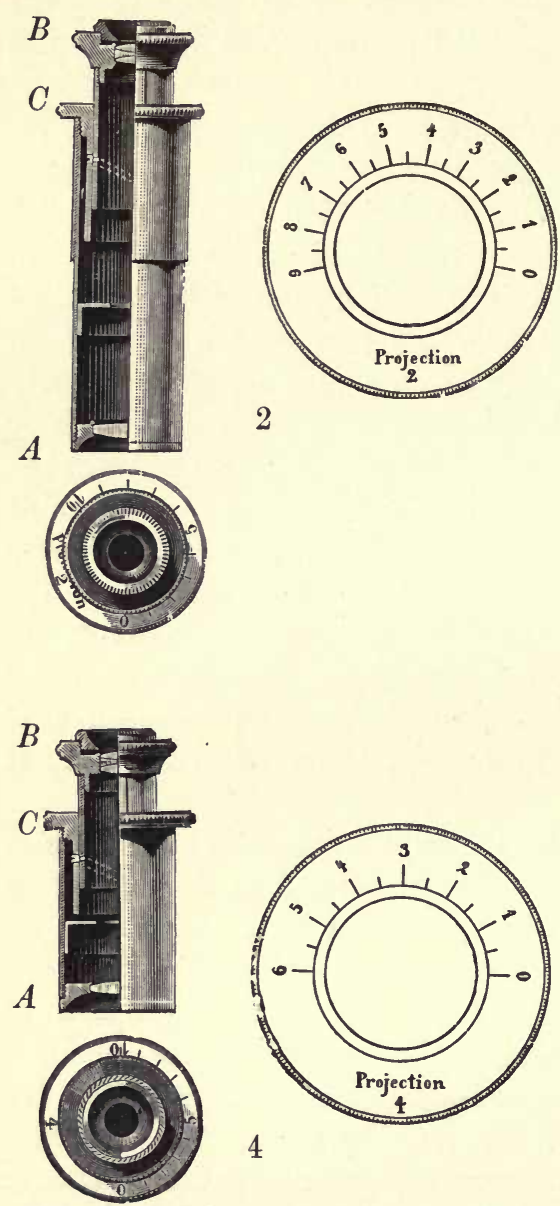

Fia. 12.-Projection Eye-pieces 2 and 4. ( $\frac{1}{2}$ full size.) directions, and is in fact turned into a broad bundle. It is due to the rough surface of the ground-glass that the light is so dispersed, and with a high-power eye-piece this rough surface is painfully evident when observing the image -in fact, any fine detail in the object is practically obliterated. If, however, this screen is given a reciprocating motion, so that the eye cannot appreciate the rough details of the ground-glass, the image is then clearly seen, and none of the disturbing specks which otherwise are evident can be discerned. Mr. Gordon has carried this method to the extent of replacing his eye-piece by another microscope, so that his eye-piece magnifies as much as one hundred diameters-an amplification of the image that is utterly impossible by any ordinary eye-piece method. It would appear that although this arrangement has considerable experimental value, its actual use in practice has yet to be determined.

Fig. 12 show's the projection-oculars of Messrs. Zeiss, Nos. 2 and 4 , in section. Thelens $\mathrm{A}$ is the collective-lens, and when the 
object is in focus produces, in combination with the objective, an image in the plane of the diaphragm in the ocular, this diaphragm being adjustable in position to sut varying cameralengths. The focussing of the image of the diaphragm which is projected on to the ground-glass screen of the camera is effected by rotating the top portion $\mathrm{B}$ of the ocular, and the amount by which this is moved can be read off on the scale which is in the position C. A reproduction of the scale is shown in the illustration in each case by the side of the respective oculars.

Messrs. Zeiss supply a tape-measure which may be conveniently utilised for determining, by measuring the cameralength only, the exact position which this diaphragm should occupy in the ocular, as indicated on the engraved scale. In order to measure the distance from the eye-piece cap to the ground-glass screen it is only necessary to determine the position of the pointer on the graduated scale of the eye-piece. The scale is divided, and reads from 0 to 9 ; each division represents an alteration in distance of $0.5 \mathrm{~mm}$. between the lenses of the ocular.

For the short-tube or Continental microscope they are made in two powers, magnifying respectively two and four diameters; and with the No. 2 ocular the shortest camera that can be used is about seven inches. With No. 4 ocular a camera as short as five inches can be used. At a distance of one metre from the eye-piece cap, the field will be about $20 \mathrm{~cm}$. across with a No. 2 ocular, and about $30 \mathrm{~cm}$. with a No. 4 .

With the ten-inch or English tube, these oculars magnify respectively three and six times, and allowance must be made for this in cases where the longer tube is in use. 


\section{CHAPTER IV}

\section{THE OPTICAL EQUIPMENT (Continued)}

Sub-stage Condensers, and Illuminating Appliances.-It is mainly owing to the efforts of English microscopists that sub-stage condensers, for efficiently illuminating microscopic objects by transmitted light, have been brought to their present state of perfection. It was for long considered unnecessary to have any arrangements for so doing that were an essential part of the optical system, and even those that were used were of a crude description, their only function being to get a larger amount of light on to the object.

It is now recognised that the sub-stage condenser is second only in importance to the objective; and such may be obtained either non-achromatic, achromatic, or apochromatic to suit the particular objective in use. They also vary in focal length, as do objectives, although within narrower limits.

It is necessary that a condenser should be, at least approximately, as accurately corrected as the object-glass, and should for critical work have as large a N.A. as the objective with which it is being used. In ordinary microscopes the non-achromatised condenser is still supplied for purposes of observation. While it fulfils its purpose well enough for visual work, or for students and others who do not undertake work of a highly critical nature, it should certainly, for photographic purposes, be discarded in favour of a more perfectly corrected combination. It is assumed that, whatever type is used, it is supported underneath the object-stage in the manner already dessribed, and has both focussing and centreing adjustments. If the additional expense of these adjustments in conjunction with an achromatic condenser is an objection, then better 
results would in general be obtained by using a reliable form of chromatic condenser with such-adjustments rather than an achromatic without them.

The simplest sub-stage illuminator is the mirror, which is fitted to all microscopes, and which is on one side plane and on the other concave. By its aid, assuming that a large source of light is being used, parallel rays are projected on to the object when the flat mirror is used, and convergent when the concave side is in use. While no further arrangement may be necessary for the examination of simple objects under very low powers, the mirror alone is quite inefficient for illuminating an object of any complexity or where other than the lowest powers are in use. The simplest form of sub-stage condenser is that known as the Abbe illuminating apparatus, introduced so long ago as 1872. This is very easy to use, owing to its having rather considerable aberrations, and to its not being sensitive to moderate variation in its position in relation to the objecteither so far as focussing or centration is concerned; it is, therefore, a convenient piece of apparatus for visual work, and may for medium powers be used satisfactorily for photomicrography. With direct light it has comparatively few limitations; but wed as an oblique illuminator-that is, with its central rays stopped out-it at once breaks down, and fails to fulfil its purpose in the way that better corrected condensers will do.

It is as important in a condenser as in an objective that the numerical aperture should be large. The numerical aperture of the Abbe apparatus does not exceed 1.0; and what is known as its aplanatic cone does not exceed $0 \cdot 45$. It will be advisable here to explain briefly the difference between the total numerical aperture and the size of the aplanatic cone in sub-stage condensers, as it is rather on the latter that the efficiency of the apparatus depends.

Reference to Fig. $13 a$ will show that, with a simple uncorrected lens, the marginal rays do not come to a focus at the same point as the central rays : the lens, in fact, is not well corrected for spherical aberration. The diagram is of course exaggerated, as there is a portion of even a simple lens that will very nearly bring the incident rays exactly to one focal point; but it is obvious that this central portion is comparatively 
small in relation to the whole. The aplanatic cone, therefore, which is the largest cone of light that the lens will bring to one focal point, is in this case a small one.

Fig. $13 b$ shows the lens system corrected for spherical aberration; the size of the aplanatic cone now approaches the total aperture of the condensing system, and the marginal rays are focussed either exactly or very nearly in the same position as the central rays. On the degree to which this is accomplished depends the size of the aplanatic cone.
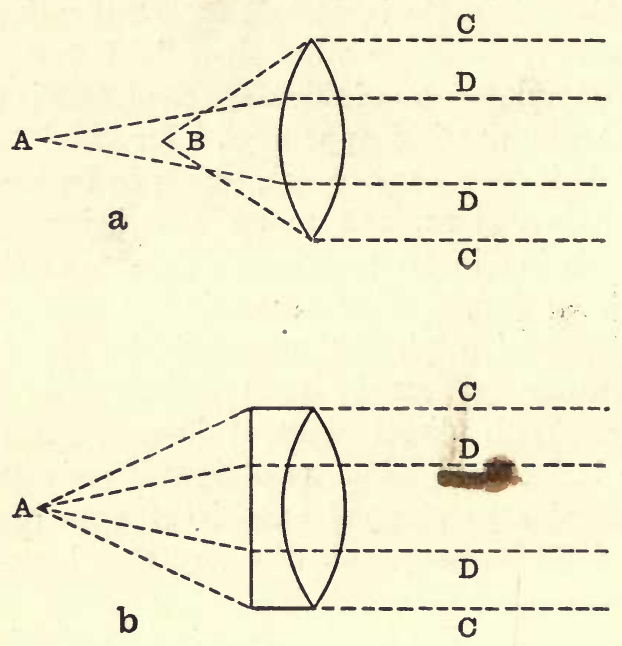

Fig. $13 a, b$.-Diagram showing Correction for Spherical Aberration.

As the primary object of the sub-stage condenser is to collect light from the illuminant and to project as much of it as possible on to the object, it is obvious that it is important for the aplanatic cone to bear as large a relation as possible to the total aperture. With condensers of the very best type the aplanatic cone is little less than the total aperture, and in some cases is claimed to be equal to it. Where the cone is not aplanatic, considerable loss of light occurs; a certain amount of light becomes diffused and is not projected into the object-plane as it should be.

Illumination may be effected by two different methods : either by what is called a solid, or by a hollow, cone of light. A 
solid cone may be of widely varying dimensions; that is, it may have a narrow or broad base, the latter being as wide as the condenser itself is capable of transmitting. The cone is narrowed by closing the iris-diaphragm below the condenser, so that the light entering the condenser is reduced and confined to a greater or less proportion of its area. To obtain what is known as a hollow cone, the central rays are stopped out by introducing below the condenser a central stop varying in size according to the objective being used. This results in the illumination of the object by an annular cone of rays, no direct light, but only that reflected or refracted by the object, entering the objective.

In cases where critical illumination is required, the image of the radiant then being projected on to the object, it is of the first importance that the illuminating cone should be aplanatic. A short table is given later showing the relation between the N.A. and the size of the aplanatic cone in well-known types of condensers.

The deficiencies of the chromatic condenser are not only its want of spherical correction, but also, as its name shows, that it is not chromatically corrected. An achromatic condenser, therefore, gives an image of any radiant that may be in use which is of a very much higher order than the simpler type. Achromatic condensers are to be obtained both dry and oilimmersion. The dry ones have total apertures, usually of $1 \cdot 0$, and an aplanatic aperture varying from 0.9 to 0.95 . They also vary in focal length ; in fact, to obtain such a high aperture it has been found necessary to make them of short focus. The average focal length of a condenser of this type is one-fifth of an inch. The result is that the image of the radiant as seen in the microscope is small, and is not usually sufficiently large to illuminate fully the field with any other than a high-power objective. To meet this difficulty most achromatic condensers are made so that the top combination may be removed, the effect being that the focal length is increased. The image of the radiant is therefore spread over a larger area, and consequently will evenly illuminate an object with a medium-power objective. This is a perfectly satisfactory method with most sub-stage condensers either dry or oil-immersion, and their efficiency is not substantially lowered thereby. 
The removal of the top combination leads to a lowering of the numerical aperture; but, as this expedient is only necessary with lenses of medium power, their aperture is correspondingly lower, so that no loss results from the alteration. Among the best at present to be obtained is an oil-immersion condenser made by Messrs. J. Swift \& Son. It has a total aperture of $1 \cdot 40$, and an aplanatic aperture of 1.30. Its focal length is $\frac{1}{4}$ inch. With the front hemispherical lens removed it may be used as a dry condenser with a N.A. of $0 \cdot 62$. Another worthy of note is a similar one made by E. Leitz, of the same N.A., but the aplanatic cone nearly equals the

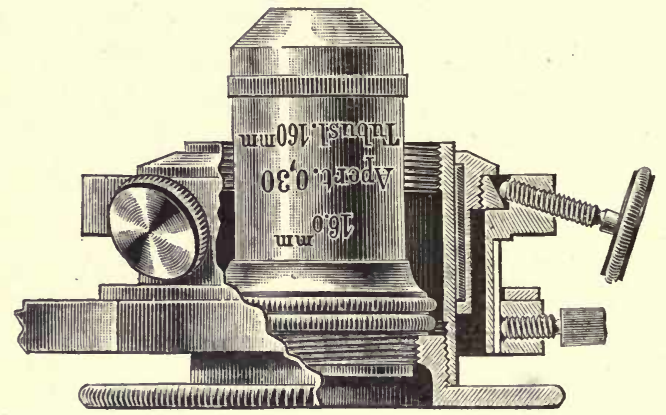

FIG. 14.-Zeiss Centreing-collar for Microscope Objectives used as Condensers. (Full size.)

total aperture. Both these condensers are capable of fulfilling the most exacting requirements.

The acme of perfection is to use as sub-stage condenser an objective exactly similar to that which is on the microscope; and Messrs. Zeiss supply a special arrangement for carrying such an objective below the object-stage (Fig. 14). In this it will be seen that the centreing-collar is the same as that in the centreing sub-stage. In place of the condenser, a short sleeve is screwed into a collar, and the latter carries the objective. The illustration shows a $16-\mathrm{mm}$. apochromat in position. The upper internal screw in the carrier is for short-focus objectives in short mounts, which would otherwise not focus sufficiently closely to the object-stage. While this is an entirely satisfactory method, it is so expensive as to limit very largely its possible use, and the condensers at present to be obtained are so perfect optically that the 
use of objectives as sub-stage condensers is practically unnecessary. There is the further objection that high-power lenses are of too short focus to obtain critical illumination through an ordinary object-slide.

Condensers which have been termed apochromatic have been brought out by one or two makers. The use of this name would not appear to be strictly justifiable, and probably arises from the fact that fluorite, and other glasses similar to that used in the construction of apochromatic lenses, have been utilised. As these condensers do not equal the correction of the apochromatic lenses, for reasons that need not be fully entered into here, they are really not entitled to be so called. At the same time they are usually extremely good, and their performance is equal to that of a high-class achromatic condenser at its best.

It may be taken as an axiom that the illumination of an object should be effected with as large a solid cone of light as possible. No objective - except when used for the observation of preparations such as bacteria or similar objects-will stand illumination with a cone of light equal to its own numerical aperture. The effect of too large a cone is easily seen, as the object appears to be flooded in light and there is a general lack of contrast over the whole field. When this is the case the sub-stage iris-diaphragm should be contracted until the image appears to possess greater contrast and until fine details are clearly defined. If this process of narrowing down the cone of light is carried too far, diffraction-fringes will appear round the components of the structure of the object; and in no case should this point be reached. The result of narrowing the cone of light by reducing the aperture of the condenser is in effect the same as that obtained by reducing the aperture of the objective. If carried to excess, the size of the diffraction discs, or what are described by Mr. J. W. Gordon as antipoints, 1 are so increased as to overlap; serious interference to the definition then results. This will be better appreciated if it is remembered that where broad beams of light are passing through an object, the amount of diffracted light in relation to the whole is not large, and it is not therefore sufficient to interfere with the general definition. Where a very narrow beam is passing ${ }^{1}$ Principles of Microscopy, by Sir A. E. Wright, p. 106. 
through a narrow slit-which in effect is the condition that holds in objects with fine structure illuminated by narrow beams - then the amount of diffraction becomes much larger in relation to the total amount of light actually passing.

It will be found in practice that the numerical aperture of the condenser should not exceed from two-thirds to threequarters of that of the objective ; usually a two-thirds cone will be sufficient. It will be understood that this does not mean that the whole aperture of the objective is not being utilised, for each detail of the object is producing its own diffraction image, and throwing broad cones or fans of light which are picked up and utilised by the objective. Consequently the effective working aperture is, in reality, greater than the aperture of the substage condenser itself.

The proportion of the aperture of the objective which is being filled by the condenser may be approximately determined by removing the ocular from the microscope and looking down the body-tube. If the aperture of the condenser equals or exceeds that of the objective, then the back lens of the latter will appear to be filled with light; on the other hand, if the condenser aperture is less than the objective, there will be a greyish-black marginal zone, and in the centre of this a brightly illuminated area. The relation of the diameter of this bright area to the whole objective aperture indicates what proportion of the total available aperture is being used.

Achromatic condensers are now made either dry or oilimmersion in the same way and for the same purpose that objectives are so made. It will be advisable for the photomicrographer to obtain a condenser of the highest possible aperture, since, as already pointed out, he can always reduce it with the iris-diaphragm; or he can for low-power work remove the top lens of the condenser-combination, and so obtain one which will illuminate a larger field, and which is therefore suitable for use with medium or low powers.

For work of the most critical nature the image of the radiant may be projected and carefully focussed in the plane of the object; but for many purposes, especially when photographing with radiants of small area, this particular method of obtaining critical illumination may be departed from. In focussing the image of the radiant, however, care should be taken that where 
it does not exactly coincide with the plane of the object, the apex of the illuminating cone falls above the object; the object, in fact, then occupies a position within the solid cone of light itself, and not above the apex of the cone.

A fairly representative series of sub-stage condensers is made by Messrs. W. Watson \& Sons of High Holborn, and may be regarded as sufficiently complete for any class of work and for use with objectives of any power. Most makers now have a series which will fulfil all ordinary requirements, and the table here set out may be taken as representative of what can be obtained in this direction. Messrs. Watson have somewhat specialised in sub-stage condensers, and their list (Table III) probably represents as wide a range of productions in this direction as can at present be obtained.

\section{TABLE III}

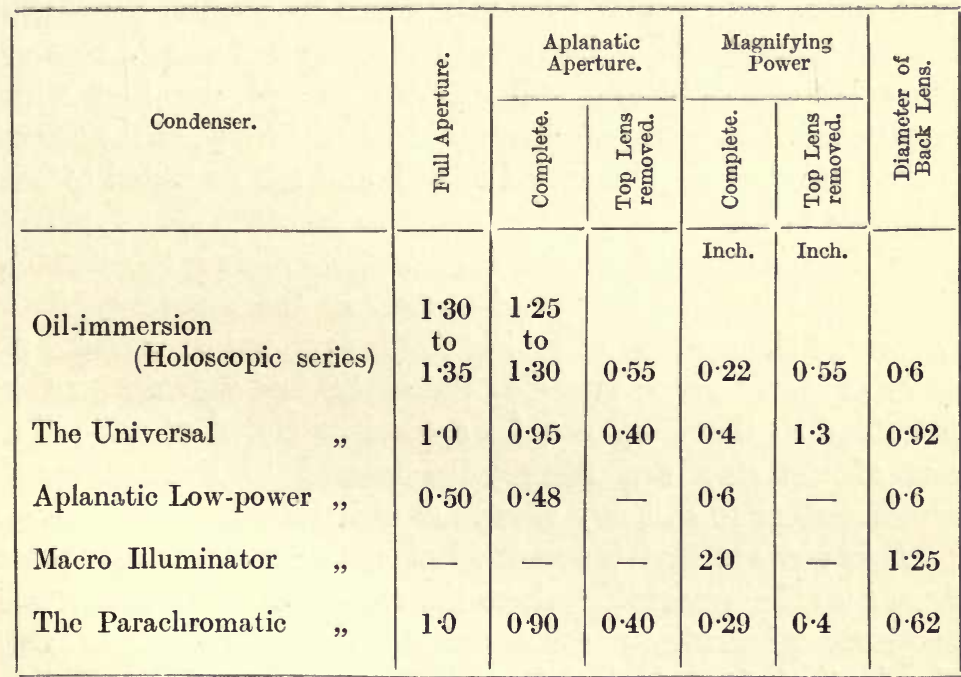

For the illumination of unstained or living objects, some method of dark-ground illumination may be desirable. Such methods depend on the utilisation of a hollow cone of light or annular illumination. To obtain this there are various appliances in use, and some of these date back to the early days of microscopy. The simplest is that known as the 'spot lens,' an arrangement only suitable for low-power work, and having 
no pretensions to be regarded as an accurate method of illumination. It consists merely of a condensing-lens, between which and the mirror of the microscope a black stop is introduced; the result is that any object placed in the position of the focal point of the lens is illuminated only by oblique rays the central direct rays being obstructed. The proportion of the total light condensed on to the object which the central spot cuts out must be greater than the aperture of the objective itself. Hence, on the assumption that the object is mounted in a medium having a refractive index substantially different to its own, any object placed at the apex of this illuminating cone shows up as a bright object on a dark ground.

Two other methods for effecting the same purpose are the use of a paraboloid, either dry or oil-immersion, or the introduction of a black central stop into or behind the ordinary substage condenser. The paraboloid, unless it is one of the modern type which has recently been introduced by Messrs. Zeiss, has become practically obsolete; but the method of using a stop in the sub-stage condenser still remains one of the best with low- or medium-power objectives. The advantage of the latter is that the stop may be varied at will, and can be suited to the objective in use.

A contrivance of recent introduction allows the size of the central stop to be varied-much in the same way that the size of the opening of an iris-diaphragm can be varied. A series of leaves are arranged at the centre and underneath the sub-stage so that they can be opened or closed at will; the size of the stop can thereby be altered to meet varying conditions, or to suit any change of objective.

It is obvious that the method of using the sub-stage condenser is only serviceable where the condenser has a numerical aperture substantially greater than the objective, as the illumination depends largely on the excess aperture of the condenser. Unless the excess is sufficient to transmit light enough to fully illuminate the object, and for it to be easily appreciated visually, then the method is unsatisfactory. This condition does not obtain with paraboloids of the older type, since to get the best results the latter should be suited to the objective. The stop used should in any case be of such a size that it obscures more than the central two-thirds 
of the total aperture of the objective; the illuminating area, therefore, consists of an annular zone amounting to approximately the remaining one-third of the aperture. In addition, any aperture that the condenser may have in excess of the objective aperture may be utilised.

To obtain the maximum illumination and the best result, the size of the central stop must be exactly suited to the objective. Most makers supply a series of these with their sub-stage condensers, but Messrs. Zeiss now have them marked to suit any particular objective. The consequence is that dark-ground illumination may be obtained with a condenser of either 1.20 or 1.40 N.A., and the stop that should be used with any particular lens is clearly marked as such.

In the latter case, the stop is supported in the condenser by a star disc, which is inserted into the diaphragm carrier, and which has a central button pointing upwards. Each stop has a small central hole which drops over the button. It is important to see that the condenser is exactly centred, as on this depends the accuracy of the result. The iris-diaphragm should be fully open except when using low-power objectives, with which a better result is often obtained by partially closing it. This is however only a matter of ordinary observation; the best effect is easily seen and the appliance can be regulated accordingly.

The best condenser for the purpose is an oil-immersion having a N.A. of $1 \cdot 40$, and with this arrangement objectives as high as a one-sixth inch may be satisfactorily used. The source of light must in this case be so projected that if the plain surface of the mirror on the microscope is used, it is illuminated with a broad beam of light; or, in the case where the microscope is in a horizontal position and the mirror therefore thrown out, that the back lens of the condenser should be completely filled with light. This is of the highest importance, as it is obviously on the use of the marginal portions of the condenser that the illumination depends, and this part therefore must be completely and evenly filled. It will be found that it is necessary to bring the condenser close up to the undersurface of the object-slide; and to obtain the best results the thickness of the object-slide must be exactly suited to the condenser. It is important to see that the central stop is strictly at the optical centre of the condenser, since any want of centration will largely 
depreciate the resulting image. It is also important to have an object-slide that is as free as possible from dirt, scratches, and impurities or irregularities in the glass ; any such become equally as visible as the object itself. Even if they are not in the exact object-plane, they result in considerable general diffusion of light which only interferes with the optical perfection of the image. To obtain the best results, the thickness of the objectslide should be determined, and the condenser should be specially made and corrected for its particular thickness.

In setting up the condenser and putting the cedar-wood oil between it and the object-slide, it is important to see that no air-bubbles are included, as these would have an even greater depreciating effect than any foreign substance or defect in the object-slide itself. The same remarks would apply to coverglasses, which should be perfectly free from defects, and their thickness must be that for which the objective is corrected. In the case where the objective has a collar-correction, the correction for thickness must be made to suit the cover-glass in use.

The object itself must be mounted in either water or oil, no result being obtainable if it is mounted in air, or in any turbid or opalescent medium. It should be as thin as circumstances will permit.

It is usual with annular illumination to employ an objective as low in power as possible, the necessary further amplification of the image being effected by the use of higher power oculars.

For photographic purposes a high-power source of light is essential ; and, where the exposures are to be short, then the only practical light is an electric arc, or a high-power lime-light. The correct centration of the light in relation to the optical system will, of course, be carried out with the same care as when photographing any other microscopic object.

It may sometimes happen that the amount of light being transmitted by the marginal portion of the condenser is greater than the object requires, and the result is seen in the image being generally fogged and with a hazy appearance over the whole object. This may be counteracted by closing the sub-stage irisdiaphragm until the illumination is as desired.

Another method of dark-ground illumination is obtained by using a suitable condenser and by stopping out the central 
rays by a fixed stop in the objective itself. As, however, this method is to a large extent superseded by the paraboloidal and similar sub-stage condensers lately introduced, it would not appear to be advisable to adopt it, for it involves a permanent alteration of the objectives. One portion of the optical combination of the objective requires to be ground away, or a permanent dark stop fixed; so that the lens as it stands is useless for ordinary observation. To render it useful for ordinary work, a special front-lens must be provided which
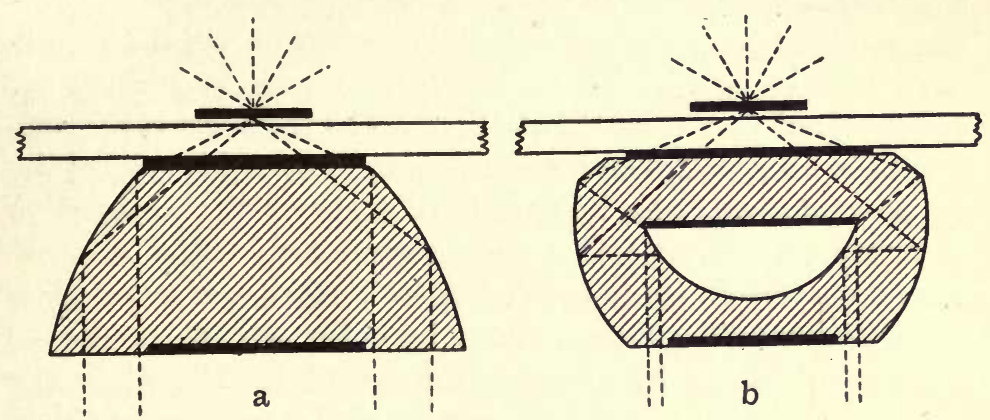

FIG. 15.-Diagram of Path of Rays (a) in a Paraboloidal Condenser; $(b)$ in Dark-ground Illuminator with Spherical Surface.

is interchangeable with that suitable for the dark-ground illumination method.

The best modern appliances which are particularly suited for use with high-power dry lenses, and which may be used for the illumination of living bacteria or similar unstained objects, are the Zeiss paraboloidal condenser, or one by Leitz, similar in purpose but different in design.

The Zeiss arrangement (shown diagrammatically in Fig. 15 a) is an improved oil-immersion paraboloid, practically the same in design as has been in use for many years, but mathematically calculated to yield a perfect image, which the earlier paraboloids certainly did not produce. The apparatus is calculated to eliminate both spherical and chromatic aberration. The illumination is effected by internal reflection of the light within the paraboloid, and relies but to a very small extent on refraction. 
Reference to the figure will clearly indicate the path of the rays, and it will be seen that the object lies at a point where the whole of the light is condensed which the paraboloid transmits. This apparatus is simple in use, providing that certain essential points are carefully attended to. Concentration must be carefully carried out, and Messrs. Ziess recommend that the objective should be supported on a centreing nose-piece in addition to the ordinary sub-stage centreing arrangement of the condenser. If, however, the sub-stage itself is carefully centred, it would not appear that the objective requires any such adjustment; but in some microscopes the centreing substage will not carry the paraboloid. The thickness of the objectslide must be exactly that for which the condenser is designed, and must not vary more than $0.1 \mathrm{~mm}$. The thickness of the slide should be between $1.3 \mathrm{~mm}$. and $1.4 \mathrm{~mm}$., and the thickness of the cover-glass should also be exactly that for which the objective is corrected. As in other dark-ground methods, when used with high powers exact correction for thickness of cover-glass must be carefully effected, either by collar adjustment in the objective itself, or by alteration of optical tube-length. The same precautions as to the elimination of any extraneous object or defects in the object-slide or cover-glass must be attended to here as in other dark-ground methods. This illuminator, having greater efficiency than any yet described, is more sensitive to any disturbing influence than any method in which the ordinary sub-stage condenser is used. A strong source of light is required; but for visual work a Welsbach incandescent gaslight is sufficient. For photographic work a more powerful illuminant is necessary; but unless instantaneous exposures are required, a one-ampere Nernst lamp, or some similar illuminant, will answer most purposes.

A condensing lens must be used between the source of light and the paraboloidal condenser, so that its posterior surface is completely and evenly filled with light. Messrs. Zeiss recommend a large spherical glass flask of some seven or eight inches diameter, which is filled with water and is used as a collectinglens. The advantage of this is that it absorbs any heat-rays that might otherwise be a cause of disturbance; and at the same time a coloured solution may be introduced so that the condensing-lens becomes a light-filter as well. A one-sixth- 
inch or 4-mm. apochromatic objective may be used with this illuminator; but if an oil-immersion objective-such as a one-twelfth-inch or 3- or 4-mm. apochromat-is preferred, then the objective itself must be reduced in numerical aperture. This is most simply effected by putting in one of the small cone fittings which drop inside the objective itself, and which have already been described as a method of efficiently reducing the numerical aperture of an objective.

The dark-ground condenser of Leitz is shown diagrammatically in Fig. $15 b$, where the path of the rays is indicated. It will be observed that the method depends on total reflection within the apparatus itself, the light being ultimately condensed on to the object; the object, in fact, occupies such a position that the whole of the light is focussed on to it. It is as sensitive to all variations of object-slide, cover-glass, \&c., as the Zeiss apparatus, and in use all the points already enumerated should be most carefully attended to. The results to be obtained with it are in every respect most satisfactory, and, owing to its construction, it is possible to obtain an even more exact concentration of the rays and a higher degree of correction than with the paraboloidal form.

The ultra-microscopic method of Siedentopf is not so clearly of use in photo-micrographic work. It is intended for the illumination of ultra-microscopic particles only; and, as these usually exhibit Brownian motion to a great degree, the possibilities of obtaining photographic records are generally slight. Further, as the images obtained are themselves not representations of the structure or detail of the object, but are mere diffraction dises, which only suffice to indicate the presence of an object and not to give a representation of its form, any further description of it here would appear to be unnecessary.

Another ingenious method of obtaining dark-ground illumination is that designed by Mr. J. W. Gordon, which differs essentially from those already described. In this method, illumination of the object is effected by a solid cone of light in the ordinary way, and the central rays are obstructed either at a point between the objective and the eye-piece, or at a position above the eye-piece known as the Ramsden disc. Either arrangement will unquestionably result in obtaining 
a bright image on a dark ground; but it is not so easily applicable to ordinary methods of observation as those previously described, and entails a special arrangement above the ocular for supporting the stop. It is also necessary that the stop itself should be very small and exactly suited in size to the conditions of observation. As these two latter points are of considerable practical difficulty, the method has not reached a stage where it can be regarded as generally applicable.

In the case of diatoms, or similar objects, it is often necessary for the illumination to be in one azimuth only, and for this purpose stops are designed which allow of the utilisation of certain portions only of the cone of light passing into the objective, the remainder being stopped out as required. As this method is of rather special application, further reference to it will be made when dealing with the methods of photographing particular objects.

It must be clearly understood that dark-ground methods do not result in any increase of resolution, although they may render visible isolated particles or the components of irregular structure that are not easily seen in other ways.

The resolving limit may be found by multiplying the N.A. of a given objective by 80,000 when monochromatic green light is used, and illumination is by a solid axial cone of light. With oblique illumination in one azimuth and with a small portion only of the peripheral zone in use, the power of resolving regular structure is increased by about one-third; but this latter method is practically only applicable to the illumination of such objects as diatoms or similar ones in which there are considerable diffraction effects in the objects themselves. The limit of visibility, so far as isolated objects are concerned, is quite a different point, and Lord Rayleigh states that isolated objects, or two bright areas separated by a dark line, may be seen if the dark line is as narrow as one-sixteenth and under certain conditions one thirty-second of the wavelength of the light used, although the resulting image does not of necessity represent the actual appearance of the object. The great factor under such circumstances is the intensity of the illuminant, and there is much reason to conclude that any isolated object may be rendered visible, even if it approaches molecular dimensions, if it is able to reflect sufficient light for 
the eye to appreciate. A keen observer will see in a microscopic image all the structure that the best objectives can show with a magnification of 750 to 800 diameters, although it may facilitate observation to amplify the image beyond this.

The methods of illumination so far described are applicable to transparent objects only ; for opaque objects totally different arrangements must be utilised. Opaque illuminators, of whatever description, are placed above the microscope stage; in the case of low powers the position usually is between the object and the objective, and for high powers as near as possible to the posterior combination of the objective. The specimen may be illuminated either by obliquely reflected or normally reflected light.

In general the principle of illumination of opaque objects is to project on to the surface of the object a reduced image of the illuminant; as nearly as possible securing the same conditions as in the critical illumination of transparent objects, except that the image formed is not the result of light obstruction, but is entirely due to the differences in absorption or reflection by the structure under observation. The appearance obtained, apart from the structure of the object itself, will vary according to the angle of incidence of the light. If low powers are used, the simplest method is to project on to the object, by means of the ordinary bull's-eye, a beam of light of greater or less obliquity to the optical axis. Assuming that the surface of the object has some irregularities of structure and is not a highly polished metallic surface, then the appearance will be one of alternate areas of light and shade. If, however, the beam is projected vertically and in alignment with the optical axis of the microscope, the appearance will be altered and in general a reversal of the light and shade will take place. The result of the alternative methods of illumination-at least, with metallic surfaces-is practically positive and negative. It is almost entirely due to the demand in micro-metallurgy for improved means of illuminating opaque objects that the existing methods and appliances were originated, and therefore the illustrations are mainly those of metal surfaces.

Fig. $16 a$ and $b$, which are photographs of polished metallic surfaces illuminated by oblique and normally reflected light, 
will better illustrate this point, and it will be seen that the appearances are practically reversed so far as light and shade are concerned.

The reason of this is fairly obvious; when the illumination takes place by projecting the light at an angle of approximately $45^{\circ}$ to the surface, the beam is reflected and no direct light enters the objective unless some alteration of its direction is caused by the structure or irregularities of the object itself. A smooth polished surface will under these

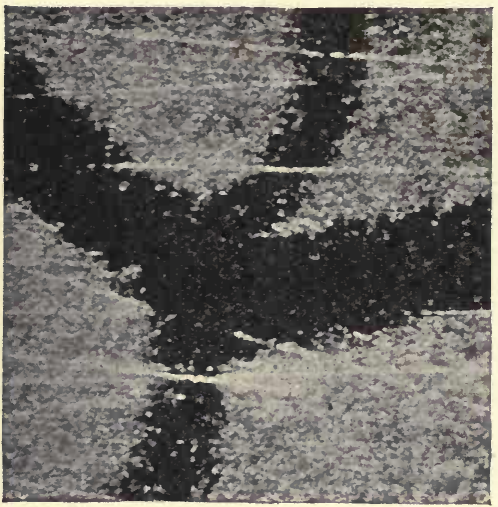

$a$

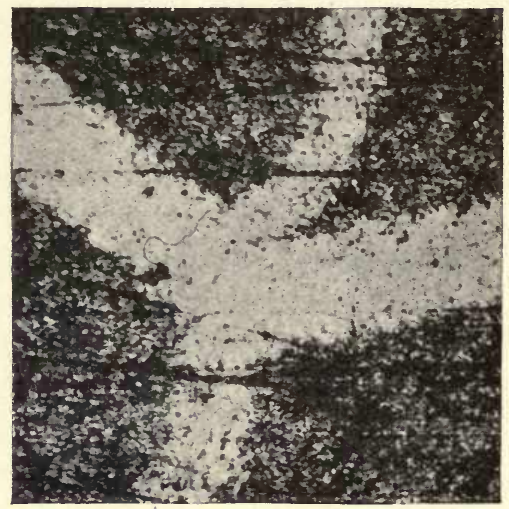

$b$

FIa. 16.-Frosted Silver-leaf Film on Glass; $(a)$ by Oblique Light, $(b)$ by Normal Light. $\times 250$.

circumstances appear practically black. If now the same object is illuminated by a vertical illuminator, the conditions are reversed; the rays are condensed on to the surface of the object by means of the front lens of the objective, and are totally reflected by the polished surface, passing up through the objective again and causing the surface of the object to appear brilliantly illuminated. The specimen selected to illustrate this point is that utilised by Mr. G. T. Beilby in his work on 'The Surface Structure of Solids,' and consists of a frosted silver surface on which a burnisher mark has been made. In both these objects there is a certain amount of light scattered; but where the burnisher has passed over, the irregularities are almost obliterated, with the result that this mark under oblique light appears black, and with vertical illu- 
mination appears as a totally reflecting surface. Even a metallic surface is, however, not perfectly opaque, so that structure becomes visible as the result in part of refraction or absorption. The absorption may be general, giving an effect of light and shade; or it may be selective, with the result that if light of a particular wave-length is absorbed, either over the whole surface or over small portions of it, the image is a coloured one. A complete comprehension of the variations in appearance to be obtained by these methods of above-stage illumination is most essential in order to interpret properly the microscopic appearances of opaque objects.

Where an object is a perfectly plain and highly polished metallic surface, both methods - the oblique and the verticalshould be used for its examination.

With the oblique method the angle of incidence is, to a large extent, determined by the focal length or working distance of the objective ; in fact, the applicability of this method depends entirely on the distance between the front lens of the objective and the object. If this distance is very small, the incident ray becomes so nearly horizontal that little or no reflection of the light into the objective takes place. For high powers, therefore, the vertical method has to be resorted to, and is practically the only one available. When using objectives of low power-certainly not higher than half an inch-the method of projecting the image of the radiant directly on to the object by the bull's-eye condenser is practicable. For opaque objects, such as foraminifera, or similar ones, where well-defined shadows can be obtained, this method works fairly well ; but it is always open to the objection that on one side a hard shadow is obtained from which there is little or no light reflected into the objective. Where no other arrangement than the bull's-eye is applicable, this can only be obviated in part by ensuring that the light is as high above the level of the stage of the microscope as practicable, that is, as high as the object-glass will permit, so that the shadows are reduced to a minimum. The bull's-eye should be in such a position that the smallest possible image of the radiant that will illuminate the field is projected on to the object.

A simple arrangement that gives good results with opaque objects, and is an improvement on the bull's-eye method alone, 
is the parabolic side-silver-reflector. It consists merely of a concave piece of metal silvered on its inner side, and this is so placed above the object that a beam projected by the bull'seye condenser is condensed by the internal reflecting surface of the reflector, and the light thus thrown on to the object. It may be attached either to the microscope body or placed on the stage, and is usually supplied with a ball-and-socket joint. The advantage of this is that the illumination, although still confined to low powers, is not at such an acute angle to the surface of the object as when the bull's-eye alone is used.

A further development of this method is that known as the Sorby parabolic reflector. It consists of a parabolic surface with an auxiliary reflector, the latter arranged so that it can be turned out of action if desired. This reflector is suitable for powers not higher than one inch, and is made to slide on to the mount of the objective. It is an improvement, and gives better illumination than the simple side-reflector already described, and is particularly applicable to metallurgical work. With both these side-reflectors a bull's-eye must be used to throw a parallel beam on to the reflecting surface, and it is essential to see that the projected parallel beam illuminates the whole of the reflector. The position of the radiant should be such that the smallest possible image is thrown on to the surface to be illuminated. The best results are obtained when the reflector is specially made for the particular objective in use-a very brilliant image then resulting. It throws light upon the object over nearly $180^{\circ}$ of arc out of a possible $360^{\circ}$, and thus illuminates a considerable amount of a solid object at one time, and lessens to a great extent the shadows that are obtained by the method in which the bull's-eye condenser alone is used. If it is made for and mounted on the objective, it is simple in use as no adjustment is required; but where only one reflector is used for lenses of varying focal lengths, it is necessary for it to be so arranged that it slides up and down on the objective until correct adjustment is obtained.

With the Sorby parabolic reflector the flat silvered mirror, which swings in and out as described, results in the light being thrown down directly on to the object, and thus a close approximation to vertical illumination is obtained. The mirror 
obscures one-half of the front lens of the object-glass, but by throwing out the auxiliary reflector it admits of a rapid change of illumination from oblique to vertical light; it is therefore very useful for ordinary purposes of observation.

Another arrangement for top-lighting - which, however, is only applicable to small objects - is that known as the Lieberkuhn. This is a concave silver reflector which slides on to the front of the object-glass ; this again must be made for the particular objective in use, as its curve must be varied to suit the focal length of the latter or more exactly to suit its working distance. The illumination is effected from below the stage, the light being projected upwards by the flat surface of the mirror on the microscope as for transparent objects. The object must therefore be so small that it allows a sufficiency of light to pass round it for the Lieberkuhn to be completely filled. The light on striking the silvered surface is reflected from all sides and condensed on to the object, and the best results are obtained when a dark stop is placed below the object, intercepting the central portion of the incident beam which is not required. The performance of this apparatus is only at its best when all the conditions indicated are fulfilled; but it then forms for low-power work a very useful method of illuminating opaque objects in cases where such illumination is required to be incident from all sides. It is obviously only suitable to low or medium powers.

The simplest and most convenient method of obtaining normal illumination with low powers is by interposing between the object and the objective a cover-glass, or piece of thin optically worked glass, which is placed at an angle of $45^{\circ}$ to the optical axis (Fig. 17). The light is thrown in the direction as shown, striking the reflecting glass surface $R$, and is partially thrown down on to the object $M$. It is then reflected from $M$ in the direction of the optical axis, and is received by the objective $\mathrm{O}$. The appliance is clamped on to the front of the object-glass by a metal ring $\mathrm{H}$, and it is only necessary to ensure that the mount of the objective is of such a size that it will allow the reflector to slip over it. This small appliance is perfectly efficient for any power where there is sufficient working distance for it to be placed in position. The illuminant with which it is used must be 
of fairly high power as there is obviously considerable loss of light.

For the illumination of opaque objects, where observation with high powers is required, recourse must be had to either one of the two available types of vertical illuminators

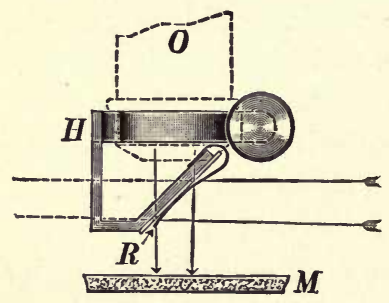

FIG. 17.-Reflecting Illuminator for Low Powers.

(Fig. $18 a$ and $b$ ). It is essential when using these vertical illuminators that the object should be either in optical contact with the cover-glass or should be uncovered-preferably the latter. It is an important adjunct in metallurgical work, and, in fact, as already mentioned, recent improvements in the illumination of opaque objects and in the method of obtaining variations of the beam of light incident on such surfaces, has been almost entirely due to its use in the examination of metal surfaces.

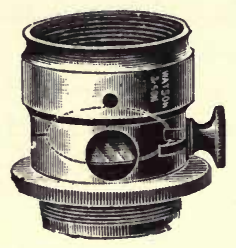

$a$

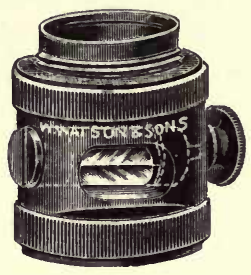

$b$

FIG. 18.-Vertical Illuminator for Opaque Objects. (W. Watson \& Sons.)

The vertical illuminator is attached to the microscope in the position usually occupied by the objective, and the objective is then screwed into its lower end. It therefore occupies an intermediate position between the objective and the ocular, but is situated as near as possible to the former. It consists essentially of either a thin cover-glass, or, in some cases, of an optically worked plane piece of glass; or, as in the Zeiss vertical illuminator, of a small totally reflecting prism. A beam of light is projected at right angles to the optical axis of the microscope so that it falls either on to the reflecting cover-glass or on to the prism and is thrown down through the objective 
itself on to the object, a critical image of the illuminant being formed at the surface of the object. The objective thus performs a double function; it acts in effect as a condenser, while also serving its ordinary purpose of image-formation and magnification of the image. The surface of the specimen may then be viewed through the eye-piece of the microscope in the ordinary way. Whether the cover-glass or the prism is the better form to use is a controversial question; but the prism arrangement certainly has the objection that one-half only of the objective is in use for the purpose of image-formation. The prism is so arranged that it projects over one-half of the back lens of objective, and this half therefore serves as the condenser; the remaining half forms the ordinary microscopic image. In general, it will be found that the arrangement in which a cover-glass is used has some slight advantage, as there is no interference with the aperture of the objective. The carrier containing the reflecting arrangement is made so that it may be rotated, and the angle of incidence of the light may be varied, within limits, at will. The radiant should be approximately eight to ten inches from the microscope, since it is only at a short distance such as this that the objective will efficiently condense the light on to the object. To obtain exactly vertical illumination the illuminator should be in such a position that its reflecting-surface is at an angle of $45^{\circ}$ to the vertical.

A considerably improved vertical illuminator has been recently brought out by Messrs. E. Leitz (Fig. 19). It consists essentially of the same arrangement as has already been described, viz. a prism vertical illuminator. It has, however, inserted at one side and in the direction in which the light is thrown on to the prism, a subsidiary tube carrying a small condensing-lens $\mathrm{L}$, which is adjustable in position in the direction of the length of the tube. To be used in conjunction with this appliance is a separate arrangement consisting of two mirrors ; the lower one $\mathrm{S}^{1}$ is adjustable in any direction, the upper one $\mathrm{S}^{2}$ is arranged at an angle of $45^{\circ}$ to the vertical. The incident light, therefore, on striking the first mirror is reflected on to the second one and thrown into the condensing lens $L$ of the vertical illuminator. The advantage of the arrangement is that the position of the source of light in 
relation to the whole apparatus is not of primary importance. The aperture $J$ has to be centred exactly so that a parallel beam is thrown on to the lens $\mathrm{L}$, but after this has been done the light may be thrown on to the mirror $\mathrm{S}^{1}$ and is variable in direction within considerable limits.

An additional condensing-lens $\mathrm{L}^{2}$ is also arranged, so that it may be thrown into action over the aperture $\mathrm{J}$ if required ; it enables the light to be condensed and a higher degree of illumination to be obtained if required. The whole appliance has been most carefully thought out, and forms at the present time one of the most convenient outfits for opaque illumination that can be obtained. At $J$ an iris-diaphragm is placed so that the width of the incident beam may be varied as desired. The arrangement can, of course, be used at will with either the prism or the cover-glass type of illuminator.

Vertical illuminators require very considerable care when used for photo-micrography, as there is great danger of a considerable diffusion of light occurring at the object or at one of the many reflecting surfaces in the optical system. For this reason the best results are obtained by using these illuminators in conjunction with oil-immersion objectives; the number of reflecting surfaces is thereby greatly reduced, and there is at least no chance of light being diffused between the front of the objective and the object, for the object is in this case directly immersed in the cedar-wood oil. While these are sometimes not of much importance for visual work they become extremely troublesome when a photograph is required, and it is absolutely essential that the exact conditions should be fulfilled under which the appliance works at its best.

A modification of the two forms described has been suggested by Mr. Rosenhain. The general construction is similar to the vertical illuminators described, but his reflectors consist of differently shaped thin silvered surfaces, either circular or annular, and they are mounted in such a manner that they may be shifted either laterally or vertically. Great importance is attached by Mr. Rosenhain to the necessity of having the reflecting surface as close as possible to the posterior combination of the objective, and where particular appearances are required the adjustments suggested by which the reflectors may be swung to one side or the other are extremely useful. 
In photo-micrography too much attention cannot be given to illumination, for on this more than on any other factor depends the perfection of the result. No apology is therefore offered for having given a rather lengthy and detailed description of practically all illuminating appliances available at the present time. In succeeding portions of this book where the method of photographing special objects is described, the method of illumination will in all cases be as fully set out as is practicable.

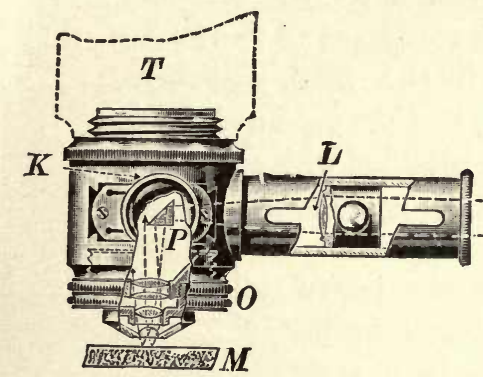

Fig. 19.-Vertical Illuminator for Opaque Objects.

(E. Leitz of Wetzlar.)

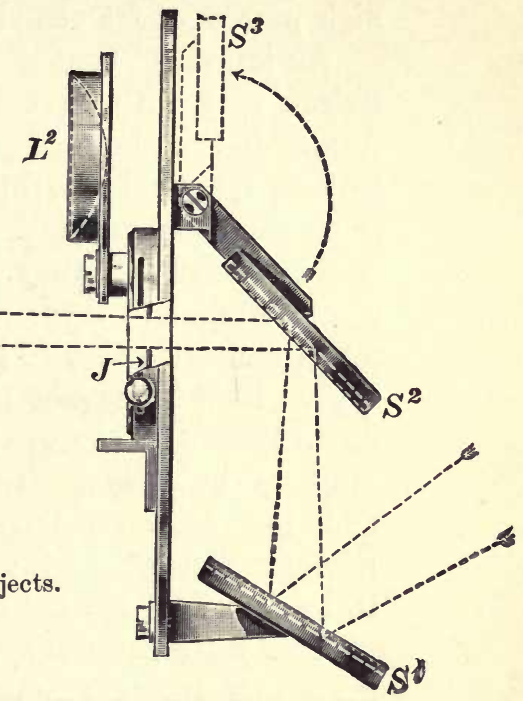

The Collecting-lens System.-The satisfactory illumination of microscopic objects for photographic purposes is not without certain difficulties which are due to the size and shape of the illuminant. The field of view must be perfectly evenly illuminated, and as a rule the illumination must be very much more intense than is the case in visual work. Most illuminants of high power are small in area; consequently where a critical image is desired they do not sufficiently cover the field, and the illumination is therefore not equal. For this reason some auxiliary collecting lenses are necessary, and a description of the principal types of these follows.

The simplest form of collecting-lens to intervene between the source of light and the sub-stage condenser of the microscope is what is known as the bull's-eye. This is a plano-convex 
lens of short focus which is placed so that it projects a practically parallel beam of light into the posterior combination of the condenser. The plane side of the lens should be turned towards the light. It is usually supplied with vertical and horizontal adjustment so that it can be placed in correct alignment with the other portions of the optical system. It is very useful for low powers, and for work not exceeding one hundred diameters it will be found of service, but, owing to the fact that it is uncorrected, it is not suited for use with high powers or for critical work.

An improved type of this appliance designed by Mr. E. M. Nelson may with advantage be substituted for the ordinary form. In this the condensing system is composed of two lenses so that spherical aberration, inherent in the single bull's-eye, is considerably reduced, and the illumination obtained by it is of increased brilliancy. It is usually supplied with centreingscrews similar to those used for sub-stage condensers, as it is much more sensitive to errors of centration than the ordinary type. It is important that the plane or concave side should be towards the source of light, as already indicated for the ordinary bull's-eye. It has proved to be a useful accessory, and will fulfil all the ordinary requirements of a photomicrographer where the more elaborate condensing systems to be described are not available.

The Watson-Conrady collecting-lens system is one that has some interesting characteristics. In this the lenses are purposely kept small, on the ground that with large lenses, especially those uncorrected for spherical and chromatic aberration, a great amount of the light passed cannot possibly be utilised in the microscope. The system consists of a carefully corrected achromatic lens of 21 inches diameter and $3 \frac{1}{4}$-inch focus, by which a sharp and enlarged image of the source of light can be produced and projected in the position of the iris-diaphragm, producing the best effect in the sub-stage condenser. As this lens comes so close to the source of light and accordingly is apt to get very hot, it cannot be cemented with Canada balsam in the usual way, but has to be put together with glycerine. The glycerine will resist the heat for a considerable time, and in the event of its evaporating off can be replaced by the user.

If reference is made to the figure illustrating the parts of 
this apparatus (Fig. 20), it will be seen that the image of the radiant produced by this condenser $\mathrm{A}$ is focussed in the plane of an iris-diaphragm C, by means of which the beam can be reduced to such a diameter as just suffices to cover the object which is being photographed, and consequently any unnecessary light is kept out of the field of the microscope. Another iris-diaphragm B is placed close to the condenser itself, and by closing this the width of the beam of light can be adjusted so that the sub-stage iris of the microscope is filled with light as required; by the combined use of these two iris-diaphragms the light passing through the condensers and reaching the microscope is therefore kept down to that actually in use when taking the photograph. An auxiliary single lens is placed close to the iris-diaphragm on which the image of the radiant is received, and this lens is of such focus that it produces a fairly sharp image of the large aplanatic condenser on the iris of the substage condenser $\mathrm{D}$ of the microscope. It therefore serves a similar purpose to that of the field-lens of an ordinary eye-piece, directing and converging the beam on to the sub-stage condenser. This apparatus has been found to fulfil all that is claimed for it, and where it is convenient to have the radiant at some distance from the microscope it is very useful. Some photographs taken by this arrangement under critical conditions will be seen among the illustrations of this book.

A complete collecting-lens system that may be used with objectives of any power is

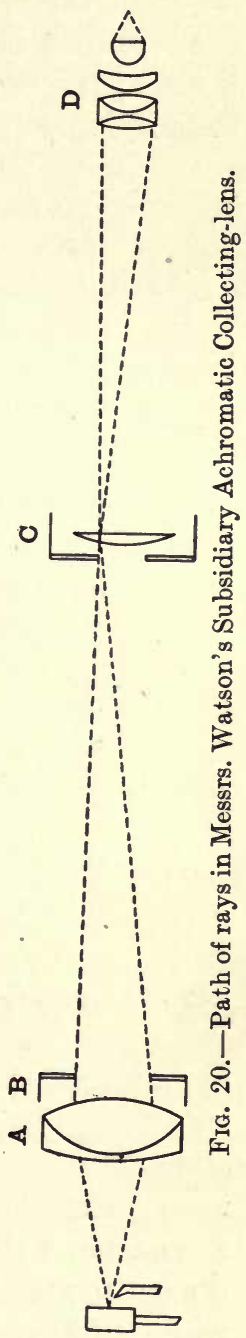
that made by Messrs. Zeiss to the computation of Dr. Kohler. In this arrangement three lenses are used, and as these are supplied by Messrs. Zeiss they are; distinguished by the Roman figures I, II, and III. By arranging these in differenc 
ways they can be used with any illuminant, whether of large or small area. For work with high powers, lens $I$ is used singly as a simple collector. It is arranged to project the image of the radiant (assuming the latter to be an arc light or some similar illuminant of small area and high power) upon the diaphragm of the sub-stage condenser of the microscope, magnifying it about four times. In adjusting this it should
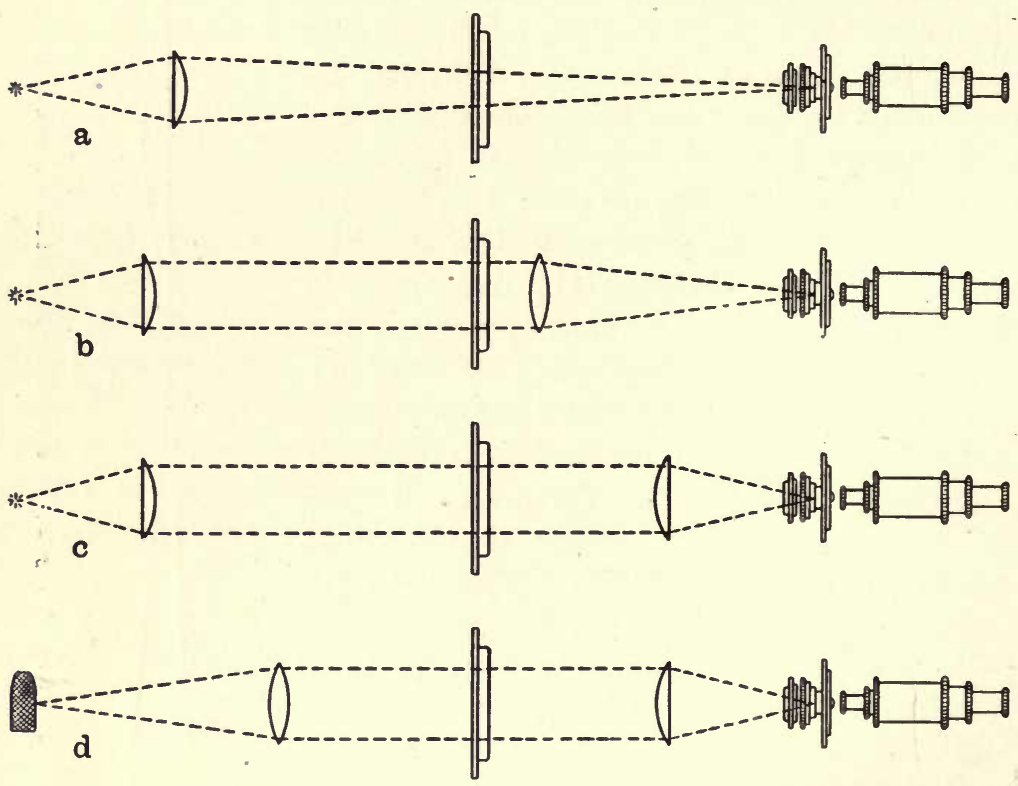

Fia. $21 a, b, c, d$.-Collecting-lens Systems of Great Light.gathering Power.

be so placed that the image of the ring surrounding the collecting-lens is projected by the sub-stage condenser of the microscope into the centre of the field of view. Fig. $21 a$ shows the arrangement of lenses, \&c., set up for high-power work. In this arrangement the image of the radiant should be so large that the entire aperture of the condenser is filled with light, as otherwise its full aperture would not be utilised. But, on the other hand, when using short-focus sub-stage condensers, the image of the radiant projected in the field of view is small, and is therefore only sufficient for illumination when using high powers.

For moderate magnifications lenses I and II are used in 
combination (Fig. $21 \mathrm{~b}$ ). Lens I is brought so close to the source of light that the latter is approximately in the focal point of the lens and projects therefore in the direction of the microscope a parallel beam. Lens II is now placed in such a position as to receive this parallel beam, collecting the light and projecting an image of the radiant on to the diaphragm of the sub-stage condenser of the microscope. In this case the projected image is smaller than where lens I only is used, and consequently the aperture of the sub-stage condenser is somewhat reduced, but as the arrangement is for medium powers only, this is a matter of no moment.

For low magnifications with lenses from $\frac{1}{2}$ to 1 inch, where a relatively large area has to be illuminated, lenses I and III are used in conjunction (Fig. $21 \mathrm{c}$ ). These lenses are of such focal length that the image of the source of light projected on to the iris-diaphragm of the microscope is of the same size as the radiant; consequently it is only useful for the powers indicated, owing to the sub-stage condenser being but partially utilised. It is important in working any of these arrangements to have an iris-diaphragm to cut off extraneous or stray light, and it should always be placed approximately in a position as shown in Fig. 21. Such a diaphragm is advisable in any case, as it can be regulated to allow the field of view of the objective to be just filled and no more; so that extraneous light, from whatever cause it may arise, is excluded, and the effect of any internal reflections is eliminated. It will be found that by closing this iris-diaphragm and projecting an image of it in the field of view of the microscope, the illumination will be quite satisfactory, and will in general give results equal to any critical illumination obtained by the method in which the image of the radiant is projected on to the object.

In practice a rather long distance is required between the radiant and the microscope, and where several pieces of apparatus have to be in perfect alignment it is advisable to carry them on a triangular metal bar, or on some similar arrangement where perfect alignment can be maintained: The saddle stands made by Messrs. Zeiss for this purpose are in every respect satisfactory, but a method of supporting lenses 
and other apparatus on geometric slides will be described in a later portion of the book when dealing with photographic cameras. This method when used for the support of the collecting-lens system and other apparatus required for illuminating purposes will be found equally efficient, and where cost is a consideration, much more economical.

A diagram is also appended (Fig. 21 d) showing the arrangement which is necessary when incandescent gas is used as the radiant, or any similar source of light of large area and low intensity. The illustration is sufficiently descriptive and requires no further explanation.

In cases where the cost of the Kohler system of lenses is an objection, a fairly efficient substitute may be arranged by fitting up three ordinary non-achromatised lenses. These may be purchased for two or three shillings each from most opticians. Two plano-convex lenses of about 10 to 12 inches focal length, and one bi-convex of about 18 inches focus will be found suitable. They may in use be set up in the manner shown (Fig. 21), and will be found more efficient than an ordinary bull's-eye condenser. 


\section{CHAPTER V}

\section{SOURCES OF ILLUMINATION}

THE illuminant that may be adopted is perhaps of primary importance, and at least it can be said that it will to a great extent determine the class of work that may be attempted, as difficulties incidental to the use of the highest powers are considerably increased unless a light source of sufficient intensity is used.

There are at present many sources of illumination available, and these will be dealt with somewhat in the order of their intensity, beginning, that is to say, with the weakest source first. The following may be taken as those which are at present most generally used :-

Petroleum oil light.

Welsbach gas.

Acetylene gas.

Oxy-hydrogen, or limelight.

Electric light, of various forms.

Sunlight.

Magnesium light has been mentioned also by many workers, but while it may be used for short exposures, it has so many practical difficulties, that on the score both of its inconvenience and of its expense and uncertainty, it hardly deserves much consideration.

In selecting the illuminant it should be borne in mind that photographic plates at the present day can be obtained of very much greater sensitiveness to any portion of the spectrum than was formerly possible. At the same time, as will be pointed out in considering the selection of suitable photographic plates, it should also be remembered that a slow plate is desirable, 
so that, broadly speaking, the more powerful the source of light which it is possible to use, the better the result that may be expected. It is also stated by no less an authority than Professor Abbe that the more powerful the source of light, the better the resulting optical image. Powerful illuminants are usually small in area, so that, both on theoretical and practical grounds, they are desirable, since they approach more closely to that great desideratum, a point of

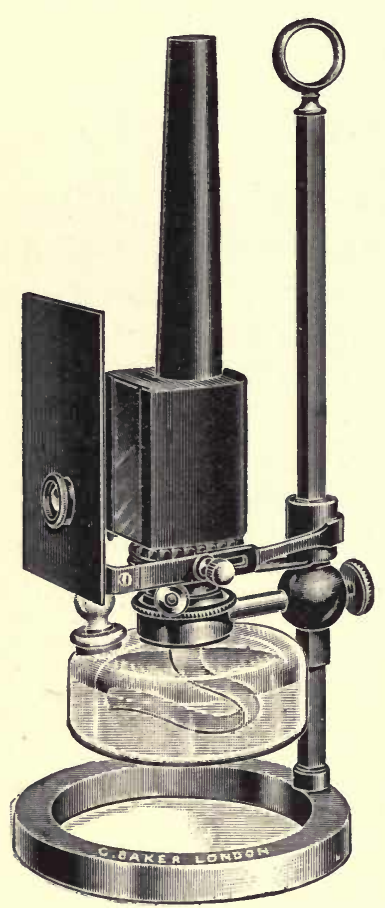

FIG. 22.-Oil lamp. (C. Baker.) light. On the other hand, for lowpower work, and particularly in cases where the objects are thin or transparent and lacking in contrast, a less powerful light will fulfil every requirement and may even give the best result.

Oil Lamps. - Probably most readers will at least commence work with an oil lamp, and if the length of exposure necessary as the result of using this is no particular objection, then a good oil lamp, carefully chosen and well constructed, will answer many purposes. The lamp should be either of the type in which the wick is broad and flat, or with a circular burner of the Argand pattern. If of the former variety it should have a wick at least one inch broad and should be so arranged that the lamp can be turned either with the broad side or the edge of the flame towards the microscope.

The usual type of microscope lamp as sold by opticians is as a rule not the most suitable, for they are generally small in the body, and altogether not solid or substantial enough in construction. One should be chosen with a vertical adjustment for centreing the light. This is usually obtained by carrying the oil reservoir on a bracket which slides up and down on a vertical rod rising from the stand, and which can be clamped in any position (Fig. 22). The writer, 
however, has used an oil lamp of simple construction for many years which has been found in every way satisfactory. The oil reservoir is a tin chamber about six inches square and one and a half inches deep. The burner is a circular one of the very best make procurable, and the light which it gives is sufficient for any work except when the use of oil-immersion objectives is necessary. No provision is made for vertical adjustment, as with such a large source of light it can be placed in position in relation to the microscope once and for all. With all oil lamps it should be remembered that the heat emitted is considerable ; so the lamp should be placed as far from the microscope as possible, and it is always advisable to interpose between the microscope and lamp, but as near the latter as possible, a blackened metal diaphragm of fairly large size with a hole in it about three-quarters of an inch in diameter.

The oil used should be of the very best quality, and should have from $2 \frac{1}{2}$ to 3 per cent., by weight, of camphor added to it, and dissolved in it. The wick itself should be evenly trinmed and thoroughly dried before it is inserted in the wick holder. All parts of the lamp should be kept thoroughly clean, as on this depends entirely the efficiency of the light and its freedom from smell. The glass chimney should preferably be of Jena glass, to lessen the risk of fracture from changes of temperature.

The chief objection to an oil lamp is, of course, that it necessitates prolonged exposures, especially where the work being done is of magnification exceeding 500 diameters. It must not, however, be overlooked that some of the best photomicrographers have obtained excellent results by its use, and some have even gone so far as to recommend it for all classes of work.

The duplex form of burner is to be avoided, as, although a higher degree of incandescence is obtained owing to the close proximity of one flame to the other, yet the inevitable dark space that occurs between the two makes its use very difficult when critical work has to be done with the edge of the flame, or where an image of the radiant in the object-plane is desired.

Welsbach Gas.-The Welsbach gas light, although it has a higher illuminating power than an oil lamp, suffers from a very considerable disadvantage; for in all attempts at using this source of light for critical work, the image of the incandescent 
gas mantle becomes painfully evident. The spectroscopic value of the light, too, is such that when using colour screens, which in most cases are absolutely necessary, the light is so deficient in certain parts of the spectrum, that it becomes in effect a very weak source of light. Where no other more powerful illuminant is available, an oil lamp will be found preferable.

Acetylene.-Of the less powerful sources of light available, acetylene gas has many advantages ; it is brilliant and yet the flame is comparatively cool. Acetylene, or to give it its scientific name, ethine $\left(\mathrm{C}_{2} \mathrm{H}_{2}\right)$, is a gaseous compound of twenty-four parts by weight of carbon with two parts by weight of hydrogen. It is evolved by the action of water upon calcium carbide, which causes a double decomposition to take place, the oxygen of the water combining with the calcium and forming lime, which remains as a residue, while the hydrogen unites with the carbon of the carbide to form acetylene. For many years, although its production was possible, calcium carbide was a comparatively rare substance, or at least so expensive to produce as to make it prohibitive as the source of acetylene. When, however, the electrolytic method of producing this substance was discovered, the whole aspect was, changed, and calcium carbide became a cheap substance, obtainable commercially in large quantities.

Calcium carbide is produced as the result of the fusion of lime and carbon in an electric furnace. It has no explosive qualities, it is non-inflammable, and in ordinary circumstances combustion does not take place. But it is essential that it should be stored under such conditions that moist air or watervapour does not reach it, otherwise deterioration sets in and some acetylene gas is inevitably evolved. The volume of acetylene gas required to obtain a given light is only about one-fifteenth that of coal gas ; there is, therefore, less risk of an admixture of air and gas resulting in an explosion, as any escape would rarely be sufficiently large. The unburnt gas has a strong odour which would at once indicate any escape; but on burning, if the gas is properly purified, there is practically a total absence of smell.

There are at present rather considerable differences in the quality of calcium carbide supplied, and it is advisable to assume that one pound of carbide of the best quality will yield not 
more than four and a half to five cubic feet of acetylene gas, and that only when a well-constructed generator is used. It is essential that the carbide be of good quality : in fact, its purity is of the utmost importance. The chief impurities to be met with in the gas are ammonia, and sulphuretted and phosphoretted hydrogen. In any generating apparatus adopted it should be seen that suitable purifying apparatus is included so that these impurities are removed; otherwise choking of the burners will result and the light will be of lower illuminating power than should be the case. The light given out on burning the gas is very actinic, and spectroscopically the nearest in value to sunlight; so that there is a very equal distribution of light throughout its spectrum - no colour in particular predominating. The consequence is that the light appears-and is, in fact-a very white one. It has an additional advantage for photomicrographic work-in that the amount of radiant heat is not very great; hence expansion of the apparatus, as the result of the heat emitted by the illuminant, is not such a disturbing factor as is the case with an oil lamp or a Welsbach gas lamp. The exposures necessary with it will be found to be about one-seventh to one-tenth of the time required with the oil lamp. The light itself is absolutely steady, and its luminosity is uniform throughout the area of the flame; so that in cases where critical illumination is required, satisfactory illumination may be obtained even with low-power objectives.

Acetylene Generators. - The generator adopted may be either automatic or non-automatic. By automatic generators are meant those which have a storage capacity for gas less than the amount that the charge of carbide is capable of producing, and in which when the consumption of gas is stopped, no more water is allowed to reach the carbide, and so a further evolution of gas does not take place.

Non-automatic generators have a gas-holder of sufficient capacity to receive the whole of the gas from a given charge of carbide.

The automatic generators may be generally divided into two classes ; or at least for our purpose it is not worth while considering any others. In the first type the carbide, which is in a granulated form, is allowed to drop in quantities as required into a chamber of water underneath. These usually have the 
disadvantage that with such an arrangement the moisture that rises from the water-chamber reaches the carbide, and so causes deterioration. Further, there is usually not sufficient depth of water to wash the acetylene gas thoroughly before it passes into the container. From experience the writer is convinced that this type is not the most desirable, and that a generator of the second type is much to be preferred. In the latter the carbide is contained in a receptacle which is subdivided into small sections. The water is allowed to reach each chamber successively; so that a small charge of carbide is only acted on at a time, and the remainder is quite out of the way of the influence of water at all. Further, the gas rises through a considerable body of water, which fulfils the double purpose of cleansing it from impurities, and what is even more important, of cooling it before use. The carbidecontainer is so arranged that the material is thoroughly saturated with water ; consequently complete decomposition takes place, the whole of the gas is extracted, and little or no waste results. The container is also surrounded by water, and, therefore, a minimum of heat is set up in the process of decomposition. A regular pressure upon the gas is essential.

The generator made by the Thorn \& Hoddle Acetylene Co., for lantern and projection work, fulfils these requirements, and will be found to be a good example of the type now described.

A burner consuming about three-quarters of a foot of gas per hour, approximating to fifty-candle power, will be found the most useful. A larger burner only results in a larger flame, and this is not desirable.

Acetylene bicycle lamps have been recommended by some for this purpose, but there appears to be little to recommend them. Excepting in the very best make, they are not entirely free from the danger of explosion, and, owing to there being no cooling arrangement, the gas, which is usually unpurified, passes into the burner in a heated condition, so that rapid carbonising of the burner may result.

A very convenient, although somewhat expensive, method of using acetylene has lately been introduced by the Acetylene Illuminating Company, and is called by them 'dissolved acetylene.' In this arrangement the gas is dissolved in acetone; and is supplied to purchasers in cylinders from which it can be 
obtained at will. It is absolutely safe, and when exhaustion takes place, the cylinder has only to be returned to the Company to be refilled. Further, as the evolution of the gas does not take place in the cylinder at all, it is delivered perfectly cool and dry and at a steady pressure. Its only disadvantage is its expensiveness, as an outfit costs some ten pounds; but in some cases the possessors of motor-cars may perhaps already have the apparatus in use.

Lime-light. - Of the illuminants that are suitable for higher power work what is known as oxy-hydrogen light or lime-light will probably be adopted by a large number of workers. It is suited in its best forms for use with the highest powers, and is only exceeded by the electric-arc light in its intensity, and in the actinic value of the light produced.

Oxy-hydrogen light or lime-light is produced by raising the temperature of the surface of a cylinder of lime to a white heat, by means of a flame produced by the combustion of hydrogen and oxygen. The necessary gases are now universally supplied compressed into steel cylinders. Pure hydrogen is not used, but is nearly always replaced by compressed coal-gas. A substitute for the hydrogen or coal-gas may be a volatile substance such as ether or gasoline, and in cases where it is not easy to procure the cylinders of compressed gas, the latter is perhaps preferable. If coal-gas and oxygen are allowed to mix in certain proportions they form, if ignited, a highly explosive mixture. Neither will explode if unmixed, nor if there is a great excess of either beyond the explosive proportion. It is therefore advisable to take reasonable precautions in working lime-light, although, unless a serious escape of either gas occurs, with modern appliances danger is now practically non-existent.

The mechanical contrivance that is generally used for combining and burning the gases and bringing them into contact with the lime surface is known as a lime-light jet. There are two types of jet most generally used, known respectively as the 'mixed jet,' and the 'blow-through jet.'

In the mixed jet, which is the more powerful, the gases, hydrogen and oxygen, are intimately mixed in proper proportions in a chamber, before they issue from the orifice of the jet and impinge on the lime. 
In the blow-through jet the ordinary house gas is used, directly from any convenient source of supply, and the oxygen alone is under pressure. It has a considerably larger area of incandescence than the mixed jet, and its illuminating power is not so high. The jets in which a volatile substance, such as ether or gasoline, is used instead of coal-gas, are otherwise similar to the blow-through type.

Lime-light as a radiant has many excellent qualities. The source of light is constant, and in the mixed jet is not of large area. It is also now, as a rule, easily obtainable in out-of-theway places, where electric light is not available. It is cheap and easy to work, and quite free from danger when carefully used and its principles understood.

If we refer to the illustration of a mixed jet (Fig. $23 a$ ), it will be seen that it is constructed as follows :-

The hydrogen is conducted in at one tube, usually on the left side, and the oxygen passes through the other; the gases then pass into a chamber which is packed with iron gauze or some similar material, so that they there become thoroughly mixed. Then they pass out through the nozzle and impinge on to the lime which is dropped on to the pin in front of the nozzle in the position as shown. The gases, which are contained in steel cylinders, must be under equal pressure, and on each cylinder, therefore, some form of regulator must be placed, so that neither gas issues forth at a greater pressure than is required, and so that this pressure may be regulated and maintained. The efficiency of the mixed jet depends wholly on the proper mixing of the gases, on the maintenance of this equality, and on regularity of pressure.

In working the jet, the hydrogen, or compressed coal-gas, must be allowed to pass through first, and should then be lighted at the orifice of the jet. The flame should be sufficiently high to rise well above the top of the lime cylinder, and should not be high enough to flare. The oxygen gas should then be carefully turned on so that only a small pressure of gas comes through at the start. The immediate effect will be for the hydrogen flame to go down; some more hydrogen should then be let in and next a further supply of oxygen, each being successively turned on in small quantities until the point is reached when a hissing or roaring sound is heard. The point 
at which the best light is to be got is just short of this condition in which the hissing occurs, so that it is best to let the gases be
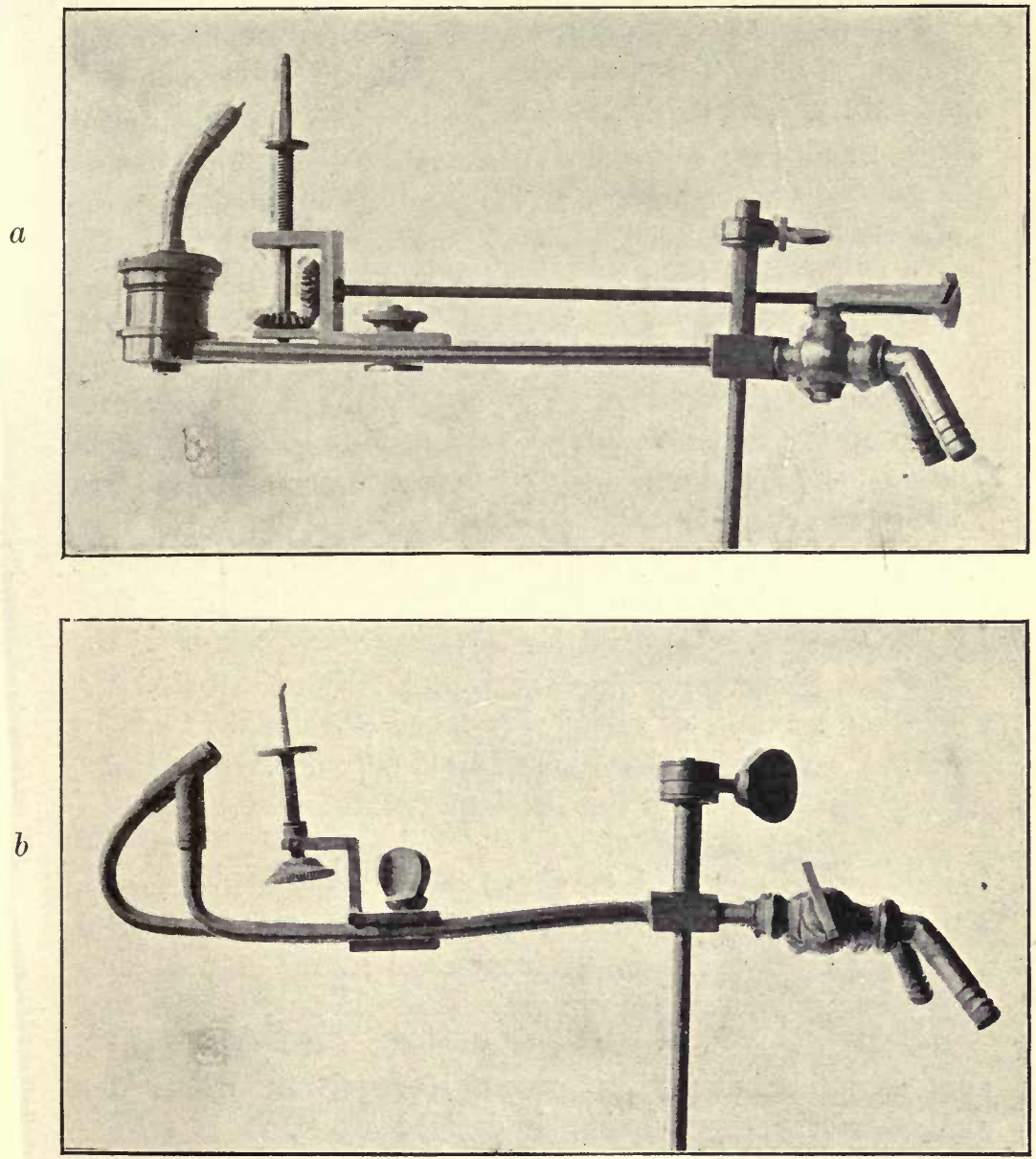

Fig. 23.-Lime-light Jets. (a) Mixed, (b) Blow-through.

somewhat in excess and then turn them both down slightly until little or no sound be heard.

The distance of the lime from the orifice of the jet is of great importance, and it should be carefully moved away from the jet until no black spot on the lime can be seen. This is perhaps 
somewhat difficult to observe directly, but any piece of glass or the surface of a condenser that may be in use at the time will act as a reflector if temporarily placed a short distance in front of the lime.

The blow-through jet is somewhat more simple to use. The tube through which the hydrogen or coal-gas is conducted may be connected up to any tap or tube which is in connection with a house supply of gas. This should be lighted up so that the flame is from an inch to an inch and a half higher than the top of the lime cylinder. The oxygen gas should then be carefully allowed to pass in from the cylinder, and very much the same condition of affairs will be noted as occurs in the mixed jet, excepting that the adjustment of the oxygen gas is the only one that is of importance. The oxygen should be allowed to pass in until it hisses or causes a distinct falling off in the light. If there is then any unnecessary excess of coal-gas it will show itself by the flame extending rather above the top of the lime, and in this case the coal-gas must be gently turned off until only a slight excess is apparent.

The 'saturator' method has advantages over either of the two above mentioned. As ether vapour is substituted for the somewhat impure coal-gas, a very much brighter light can be obtained. The writer has himself tried only one type of these, namely, that known as the Pendant Saturator, and made by Messrs. Willway \& Sons of Bristol. This saturator is arranged to work with any jet made for mixed gases. Pressure is, of course, obtained only from the oxygen cylinder. It is claimed for this method that it is the most economical means of producing oxyhydrogen light, since not only is the hydrogen substitute less costly than compressed coal-gas, but owing to the freedom from impurities, less oxygen is required. Where this method of light production is decided upon, it is recommended that the makers of this apparatus should be referred to, for they issue a very concise and fully illustrated description of it, together with instructions on its method of working.

The selection of the limes for either jet is of some importance. For the mixed jet a hard form of lime is necessary, as both gases work under pressure; and, further, as the temperature is so extremely high, pitting or excavation of the surface of the 
lime quickly takes place. This is to some extent overcome by turning the lime at intervals of five or ten minutes-the time depending largely on the pressure of the gas - so that a fresh surface of the lime is exposed to the action of the jet.

In the case of the blow-through jet, a softer form of lime can be used; as the surface of incandescence is larger and one gas only is under pressure, pitting does not so quickly occur in this type.

It is advisable to use limes that are of good quality and truly cylindrical. In putting them on the pin which supports them on the jet, it should be borne in mind that the hole should be sufficiently large to allow the lime to drop on easily; otherwise, when the jet is started, expansion of the metal results in a fracture of the lime occurring at a very early stage. Lime cylinders can now be obtained in sealed glass tubes, and it is advisable to adopt these although they are somewhat more expensive than those sold in tins. They are usually of better quality, and, further, being absolutely protected from atmospheric influences, there is no chance of their absorbing water-vapour from the air, and consequently they remain in good condition for any length of time. The glass casings need only be broken when they are required for use, and there is consequently no opportunity for moisture to reach them, or for any expansion to take place, as usually happens with limes that are not so encased.

There are substitutes for lime now to be obtained-particularly the Zirconium dises or cylinders of a smaller size than the standard lime. They are sold by Messrs. Zeiss, and have the particular advantage that they offer more resistance to the high temperature, last for a longer time, and, being of smaller size, constitute a smaller and therefore better source of light. They do not deteriorate so rapidly as lime cylinders, nor are they subject to atmospheric influences.

When purchasing either the steel cylinders for the compressed gases, or the gases themselves, it is of the first importance to go to a firm of good repute. The products of the British Oxygen Company, formerly known as Brin's Oxygen Company, may be mentioned as reliable ; their cylinders are thoroughly tested before being sent out, and in addition to this, to prevent the possibility of any accident in filling, they now make them 
with right- and left-handed screws, so that only the gas for which the cylinder is made can be put into it.

The oxygen is always contained in a black cylinder, and the hydrogen in a red one, and these are regarded by the trade as sufficient indication of the contents.

The regulator made by $\mathrm{Mr}$. R. Beard fulfils every

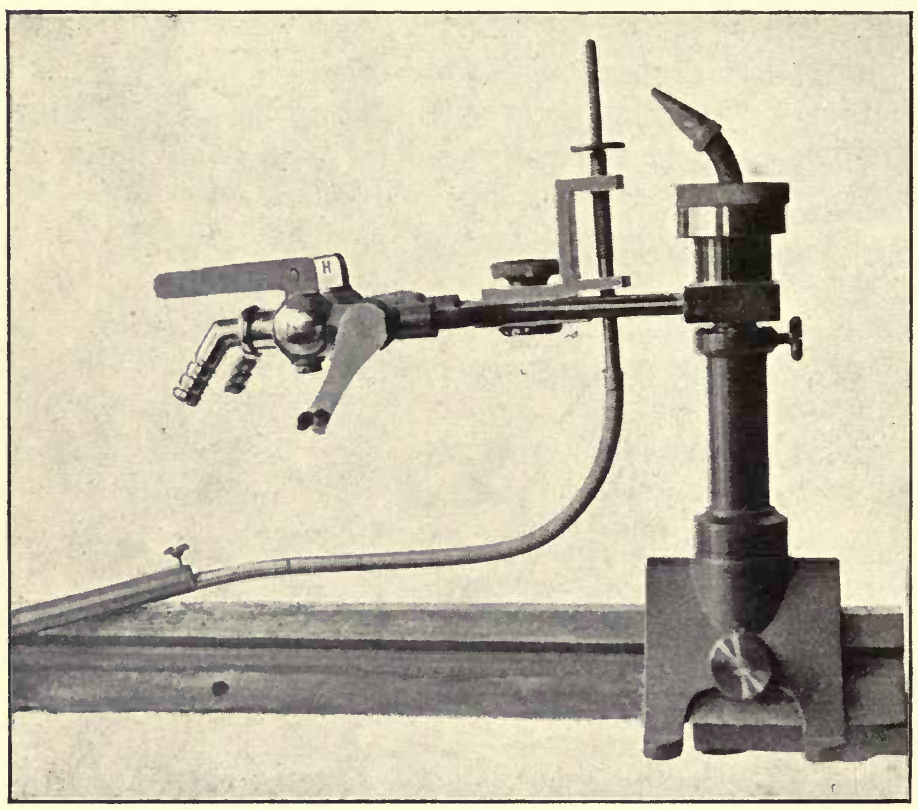

FIG. 24.-Lime-light Jet on Saddle-stand.

requirement. It has been in use for many years, and, so far as the writer is aware, without accident of any kind.

A regulator that has been used on an oxygen cylinder should never be transferred to the hydrogen one; but if two cylinders are used, a regulator for each must be kept. These are now also made with right- and left-handed screws, so that an oxygen regulator will only go on to an oxygen cylinder, and the same applies to the hydrogen.

There are a large number of lime-light jets on the market, and it will suffice to refer to two or three typical ones.

Fig. $23 b$ is a type of blow-through jet, with a movement to 
rotate the lime while the jet is burning-an adjunct which is indispensablo with all forms of jet. Fig. 24 is a type of mixed jet for use with the two gases under pressure. In this one a special cut-off (devised by Mr. Andrew Pringle) is fitted, so that the light can be turned up or down without altering the relative adjustment of the taps. This is a great convenience in photomicrography as, usually, the exposures are short, and the light is only required for short periods.

The Gwyer jet is another type for use with mixed gases, and is the most powerful at present to be obtained. In its smallest size it is stated to give a light of approximately 1500 candlepower, and the larger sizes considerably exceed this. The

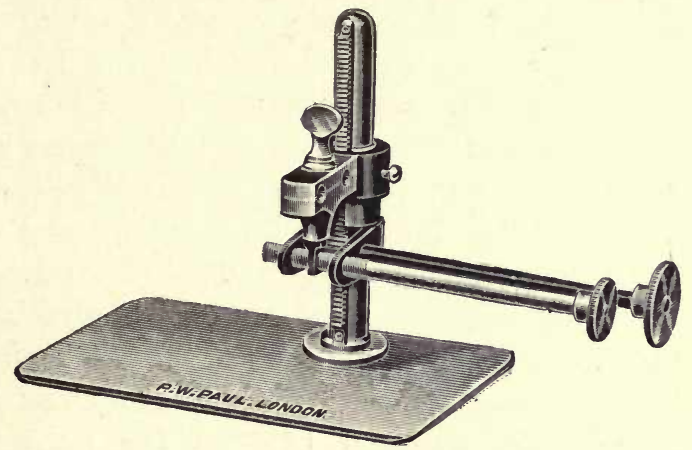

Fı. 25.-Adjustable Lime-light Tray. (Gwyer Jet Co.)

standard patterns are provided with a tap to cut off both the gases, and also with serew valves for exactly regulating the supply of gas to each tube. With the cut-off tap the gases are reduced in succession, and only a small hydrogen by-pass flame is left. The position of the screw valves is not altered, so that the light can be obtained at once in its original condition without re-adjustment of the relative quantities of the gases.

It is convenient to support any jet on a centreing-tray. This is an arrangement by which lateral or vertical adjustment can be obtained by the rotation of a milled head controlled from the back. It is so essential in microscopic work for the light to be accurately centred, that this is almost a necessity. The mechanical stand shown in Fig. 24 is a good type, and the light may be centred without fear of altering its position. Any such appliance should be well and strongly made, as otherwise, 
from the way in which the jet is usually supported, the light is easily thrown out of adjustment.

Whatever form of lime-light apparatus may be adopted, it is advisable to see that it is of the very best make. The price is usually somewhat high, but a reasonable outlay will be found to repay itself, as otherwise unforeseen troubles are almost sure to arise.

Electrical Illuminants.-These have many advantages for the purpose in view. It is now so general for a supply of current to be available in laboratories, and even in private houses, that both on the score of convenience and of economy one of the many forms of electric light is to be preferred.

Among those that are suitable for photo-micrography are the Nernst lamp, the mercury-vapour lamp, and the electric arc. Ordinary incandescent filament lamps, either carbon or their lately introduced substitutes, metallic filament lamps, such as tungsten and tantalum, are totally unsuited for this purpose. In these the filament is far too thin, and owing to its length, the source of light is not sufficiently concentrated. It also becomes very difficult to obtain critical illumination, in which the image of the radiant is projected into the objectplane of the microscope. Even though a diffusing medium, such as ground-glass, be used between the light and the microscope it does not answer well, for when the initial source is of low intensity, as it is in all filament lamps, the loss of light then becomes a really serious factor.

Nernst Lamps.-A convenient form is the Nernst electric lamp, as its current consumption is small, and yet it has a comparatively high initial luminosity. It requires no special arrangements for electrical supply, such as extra thick wires which are necessary to carry the current of an are lamp. Its fundamental principle is that the metal oxides of certain rare earths will not conduct electricity at normal temperatures, but will do so quite easily if their temperature is first raised, the result being a brilliant white light. Its spectrum is such that every requirement is fulfilled when selective colour-screens are used.

The filament, in the form of lamp which is to be recommended, is a small straight rod varying in thickness according to the current used. It will be advisable to consider only the form which takes a current of one ampere, as in this the filament 
is fairly thick and straight, and the light is then of sufficient intensity and of suitable area for work with high powers (Fig. 26).

As the filament will not conduct electricity until its temperature is raised, the preliminary heating may be effected by holding it over a small Bunsen flame, or a separate electrical

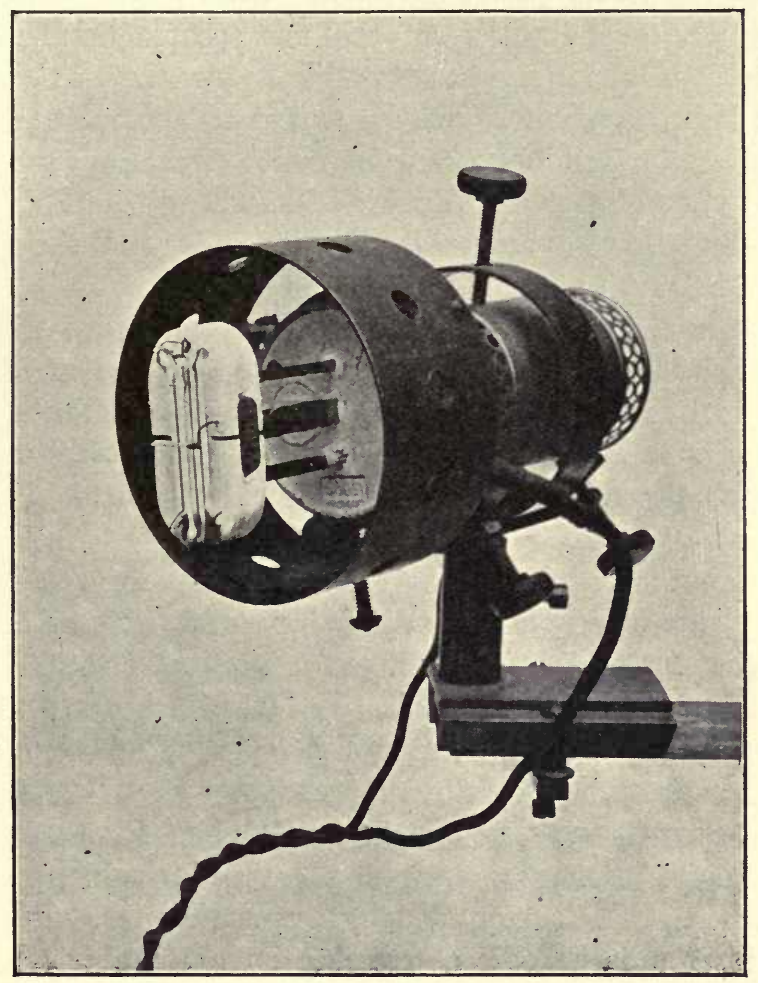

Fia. 26.-Nernst Electric ' Luna' Burner.

heater is mounted close to it. On allowing current to pass, the temperature of this heater is at once raised. It is in close proximity to the illuminating filament, and consequently the temperature of the latter is raised also. When the temperature of the filament reaches from $600^{\circ}$ to $800^{\circ} \mathrm{C}$. its conductivity is increased sufficiently to allow current to pass through it. By an automatic arrangement the current is then cut off from the heater and passes only through the illuminating filament. The lamp does not light immediately the current 
is switched on, owing to the necessity for this preliminary heating, but this does not present any particular disadvantage for microscopic work. The time required for the lamp to attain its full lighting efficiency is only about thirty to forty seconds.

With the one-ampere Iamp, already mentioned, the filament is sufficiently thick to form a source of light that will evenly illuminate a great portion of the microscopic field, and be sufficient at least for moderate or high powers.

The lamp must of course be suited to the voltage of its circuit, but the candle-powers for which the lamps are made and sold vary almost directly with the voltage of the supply. Hence a lamp for 100 volts will only give about half the candlepower of a lamp for a 200 -volt supply.

There is a regulating resistance placed in series with the filament, as it is rather liable to fracture with sudden changes of electrical pressure, and consequently this resistance has a steadying effect. The resistance absorbs about twenty volts, the remainder of the electrical energy being utilised in the production of light.

The lamp is supplied in a very convenient form which can be easily supported on any adjustable stand, such as a retort stand, the filament being then in a vertical position. The adjustable lime-light trav already described with vertical and horizontal movements, may also be easily adapted, and is the most satisfactory form of support.

The type of lamp that should be purchased is that known as the 'Luna' Nernst lamp, and not those in which the filament is bent, or is surrounded by a spiral heater. Its initial cost is low, and the cost of running is comparable with any ordinary carbon filament lamp, its efficiency being very high.

The candle-power measured in the direction of the axis of. the lamp is as follows :-

\begin{tabular}{|c|c|c|c|c|}
\hline Voltage. & & $1 \mathrm{Am}$ & o. ' $\mathrm{L}$ & Lamp. \\
\hline 200 & $=$ & 200 & cand & ower \\
\hline 220 & $=$ & 250 & , & \\
\hline 240 & $=$ & 274 & " & \\
\hline 260 & $=$ & 296 & , , & \\
\hline
\end{tabular}

It is essential in connecting up that the negative pole of the source of supply should be connected to the negative 
pole as marked on the lamp; otherwise the life of the lamp is considerably shortened.

Which of the wires making connection with the lamp is the negative pole may be determined by using what is known as pole-finding paper, to be obtained from any electrical engineer's supply store. Or a simple method is to moisten a piece of filter-paper with a solution of bromide or iodide of potassium, and then quickly touch the paper with the ends of both wires. Great care should be taken in doing this that the ends of the wires are not allowed to touch each other, and they should be so held that they are from an inch to an inch and a half apart when they touch the paper. The negative wire will then, where it touches, cause a small brown spot to appear, and this is the end which should be connected up to the negative pole as marked on the lamp.

The type known as the Projector Nernst lamp should not be selected ; in it there are three parallel filaments, but although the arrangement is very suitable for ordinary optical projection, it offers no advantage for microscopic work.

Mercury Vapour.-One of the more recent forms of electric light likely to prove valuable in photo-micrographic work, and in fact in all microscope illumination, belongs to the type of which the mercury-vapour lamp is an example.

In this method of light-production we have what is really an are light, but with the very important difference, that in the mercury-vapour lamp the gaseous are itself constitutes the source of light, instead of one or both of the solid poles as in the carbon arc. Further, the current necessary to maintain a mercury-vapour lamp is very much smaller than that required to maintain any carbon arc. The mercury vapour which constitutes the source of light is enclosed in a glass, or preferably a quartz tube, and so is not subject to atmospheric influence; it may be exactly described as an hermetically sealed mercury arc.

The production of light in this case depends on the same essential conditions as obtain in the arc light. The mercury, however, constitutes the poles between which the arc itself plays. The mercury is vapourised at the negative pole, and the vapour becomes highly incandescent as the result of its molecular activity and not, as in the arc and other electric 
lights, as the result of rise of temperature. Spectroscopically, the light consists mainly of three bright lines-one each in the yellow, green, and blue-violet regions. It also has a very extended ultra-violet spectrum; but for the purpose under consideration this is not of moment. It is obvious, however, that it is only necessary to screen off the colours which are not required with suitable coloured screens, by a method to be described later, in order to obtain a light which is truly monochromatic, and which is, or may be, of one wave-length. Even without any colour-screen it is optically more efficient than other illuminants, since owing to the absence of red radiations the mean wave-length is less. Consequently, the defining and resolving power of any microscopic objective is appreciably increased.

The type of mercury-vapour lamp supplied commercially for ordinary photographic purposes is not the most suitable for microscopic work, as the containing-tube is far too large. The lamp shown in the illustration (Fig. 27), being very much smaller in size and having a glass tube of much less sectional area, has proved to be entirely satisfactory. The current-consumption is small, and with a glass tube should not exceed one and a half amperes; so that it is a source of light that can be used with absolute safety on any house-lighting circuit, current being obtained from an ordinary wall-plug or lampsocket. It has the additional and by no means unimportant advantage, that there is practically no radiant heat; hence, if required, the lamp can be run in very much closer proximity to the microscope than is possible with any other light-source yet described. No water-cell to absorb heat need be placed between the illuminant and the microscope.

As the mercury is enclosed in a glass tube, exhausted to such a degree that its vacuum is considerably higher than that of an ordinary incandescent electric lamp, no consumption of the mercury takes place. The mercury is volatilised; but, on the current being switched off and the lamp stopped, it is at once recondensed and consequently no loss of mercury occurs. The lamps usually have a very long life, excluding the risk of accidental breakage of the tube, and their efficiency goes down but very little even after running for a thousand or more hours. This is a characteristic by no means common in electric lamps 
other than that of the ordinary are, for with most of them their efficiency tends to decrease rapidly after they have run a few hundred hours.

Fig. 27 indicates clearly the construction of the lamp as now supplied by Messrs. C. Baker of High Holborn. It consists essentially of two parts: the tube, containing the mercury,

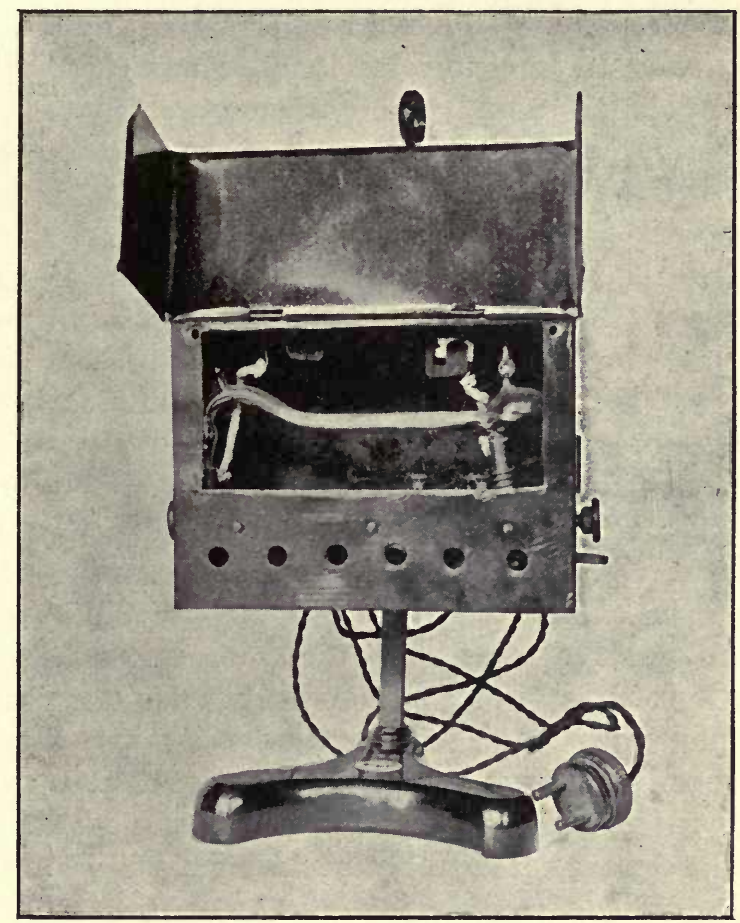

Fig. 27.-Mercury-vapour Lamp. (C. Baker.)

and a resistance, which has to be suited to the voltage of the electric supply. These are enclosed in a metal cover, and the whole is carried on a heavy base, with a square upright, on which it is adjustable for height.

To start the lamp it requires to be tilted and then quickly brought back to a horizontal position, so that the mercury flows from the positive to the negative pole and completes the circuit ; for this purpose the metal cover is pivoted in a suitable manner. It sometimes happens that the lamp does not 
start at the first tilt, and this may have to be repeated two or three times. To do away with this disadvantage Messrs. Baker now arrange that by depressing a knob, on the righthand side, some portion of the resistance is cut out. The lamp is then allowed to run for half a minute or so before the knob is released, and by this means it will be found that there is usually no difficulty in getting it to light even after tilting it once only.

It is essential in connecting it up to any elcetrical supply that its polarity should be strictly observed. The negative wire should be connected to the end of the tube which has the smaller bulb (shown in the illustration on the left-hand side), and the positive pole to the opposite end.

Should the surface of the mercury appear to boil as it retreats along the tube, the connections are not as they should be, and the plug should be reversed.

When the lamp is running, slight ebullition should be apparent at the negative pole.

It will be found that for visual work the intensity of illumination is sufficient for use with the highest powers. But for photographic work where greater luminosity is required, tubes can now be obtained of quartz or fused silica. They are practically of the same shape and dimensions as the glass tubes; it is possible to run a much heavier current through them, and with a higher current-density the illuminating power rapidly increases. With a current of three amperes, the lamp is sufficiently powerful for high-power work, and the exposures compare favourably in duration with, for instance, the blow-through lime-light jet.

The quartz tubes are, unfortunately, much more expensive than the glass ones. Were it otherwise there would be no object in using glass at all, for it would be entirely superseded by quartz. Both types of tube are made by the Bastian Mercury Lamp Company, or by their licensees, and can be obtained from Messrs. Baker.

A further modification of the mercury-vapour lamp has quite recently been brought out by Messrs. Swift \& Son (Fig. 28). This does not differ in principle from the form already described. The mercury-vapour tube itself is carried in a metal box, in the front of which is an iris-diaphragm, so that the available size 
of the source of light may be varied at will. This has in practice been found to be a considerable advantage, as the diaphragm is very close indeed to the source of light, and it is therefore possible to vary exactly the degree of illumination without altering the adjustments of the microscope. The necessary resistances are mounted at the back of the lamp, the resistance-wire being wound on vertical tubes. To facilitate the lighting of the lamp, a portion of the resistance may be cut out by the spring lever (shown on the left-hand side of the figure). In this type the resistances are not carried in the lamp body, but are separated from it. The unavoidable heating that occurs in the resistances does not therefore tend to raise the temperature of the other parts, so that adjustment of the illuminating portion may be more easily effected. The whole appliance is well made and finished, and has all the necessary arrangements for varying height and inclination. It is supplied with a set of the necessary colour-screens.

The Electric Arc.-Of the higher power illuminants

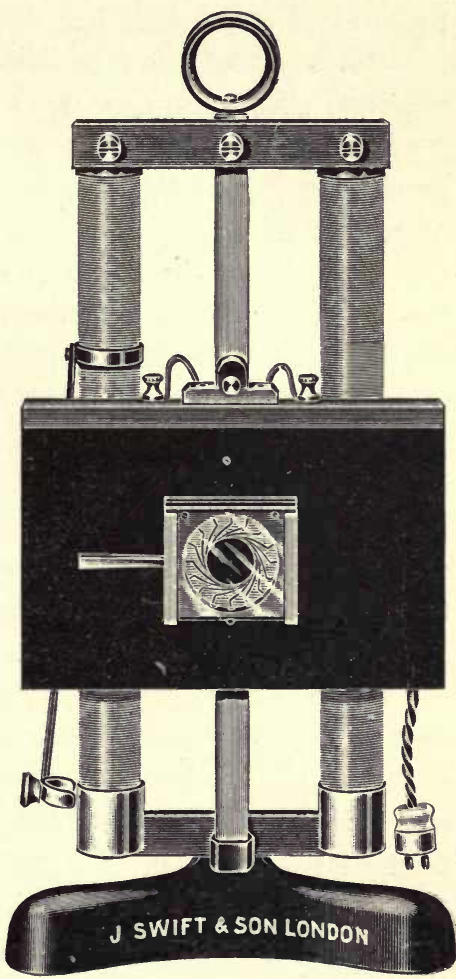

FIG. 28.-Mercury-vapour Lamp. available the electric are is, both theoretically and practically, almost an ideal one. From the point of view of practicability it is at the present day probably the most convenient, as nearly every laboratory and many houses have a supply of electricity; and from the optical point of view it has the advantage that the source of light more nearly approaches the much-desired optical point than any other. 
The production of an electric arc is dependent upon the fact that if an electric current of suitable voltage is allowed to pass between two carbon points which are slightly separated, one or both of these points becomes a highly incandescent source of light.

There are two methods of producing and maintaining an are light now in general use; one, in which some automatic method of regulating the distance between the carbon points is used, and the other which depends on a simple arrangement whereby they are adjusted by hand.

Let us for a moment consider the essential conditions for producing an arc light with continuous current. Most sources of supply of electrical energy are now such that the current is supplied at from 100 to 250 volts. It is, perhaps, unfortunate that an electric are requires only from 40 to 50 volts; so that some method of absorbing the difference between the voltage of the supply and the potential difference required between the carbons must be adopted. This is usually accomplished by putting in series with the lamp, of whatever form it may be, a resistance - that is to say, an arrangement in which some of the electrical energy is expended in producing heat. The term ' in series' means that the lamp and resistance-coil are arranged so that the current passes successively through each.

When it is required to start an arc it is essential that the two carbons should momentarily touch. Be the distance between the carbons ever so small, a current of electricity of low voltage will not pass between them unless this preliminary contact takes place. On making contact, some small portion of the extremity of the carbons is volatilised, so that on parting them again, the volatilised carbon acts as a conductor, and the electricity passes across the gap ; in so doing, it raises both points of the carbons to a condition of incandescence.

It is almost universally the practice in are lamps to place the positive carbon - that is, the one that is connected up to the positive wire coming from the electrical supply -in the uppermost position, the negative carbon being placed in the lower holder of the lamp. When an arc is started, it does not at first burn steadily, nor is the source of light a constant or regular one. This is due to the fact that, until what is known as a 'crater' is formed on the tip of the positive carbon, the 
light does not settle down and become constant. This crater is really the source of light, and in microscopic work it only is used, the negative carbon being so placed that the light from it does not reach the microscope.

The ends of both the carbons are generally tapered off, and in course of burning the tips tend to keep this shape. The crater on the positive carbon should be of such diameter that it is well confined within the limits of the tapered point-that is, there should be no tendency for the white-hot portion of the crater to go over the edge of the carbon. This will never happen if the size of the carbons is correctly chosen, but the larger the current which the lamp takes, the larger must be the size of the carbons used. When the current is too large for the carbons, the are begins to hiss, and the luminous point spreads and extends over the edge of the crater. To decrease the chance of the luminous point altering its proper position on the end of the positive carbon, the latter is always 'cored' - that is, it has a hole through its centre which is filled with softer carbon than the outer portion. The result is that this softer carbon vapourises rather

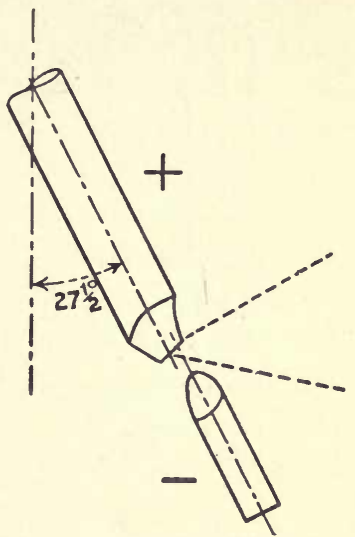

FIG. 29.-Correct Position of Electric-arc Carbons. more rapidly than the surrounding harder carbon, and the crater therefore tends to remain in its proper position.

The position of the carbons with a continuous-current are should be as shown (Fig. 29) - that is, the positive carbon should be set somewhat behind the negative one so that the crater tends to form rather towards the front and facing the direction in which the light is required. The carbons should be placed so that their front surfaces are approximately in a straight line. They should also be inclined at an angle of about $27^{\circ}$ to the vertical; at this angle two essential conditions are secured-viz. the greatest amount of light is thrown forward from the crater of the positive carbon, and practically the whole of the light from the negative is thrown to the back, and 
consequently eliminated as a second and harmful source of light.

With each current that is used-that is, with any given number of amperes - there is a critical length of arc at which the maximum light is obtained, and the greatest efficiency and constancy secured. With a current of, say, ten amperes, the best length of arc will be found to be from three to four millimetres ; but with any hand-feed method of adjustment, this can easily be determined by experiment. The arc should not be so short that hissing occurs, and should not be so long that there is any variation in the

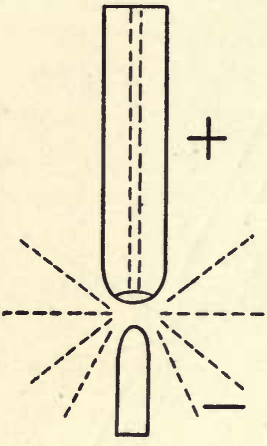

a

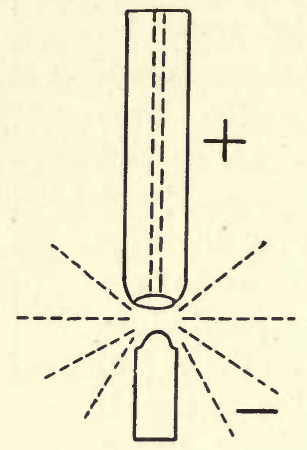

b

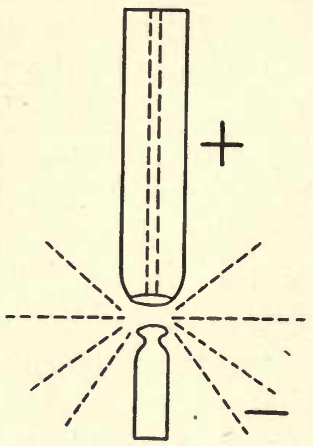

c

Fig. 30.-Appearance of Electric-arc Carbons under Different Conditions.

position of the luminous crater on the extremity of the positive carbon.

The sizes of carbons that should be used may be taken to be approximately as follows :-

$\begin{array}{ccc}\text { Current in } & + \text { (Cored) } & - \text { (Solid) } \\ \text { amperes. } & \text { mm. } & \mathrm{mm} . \\ 5 & 7 & 5 \\ 10 & 12 & 8 \\ 15 & 14 & 9\end{array}$

Observation of the form of the negative carbon is very useful in determining whether the lamp is burning properly and with a normal current supply. Under proper conditions it should have a regular cone-shaped head pointing towards the crater on the positive carbon (Fig. 30 a). If the current is 
too large, the tip of the negative carbon will become blunt and rounded (Fig. $30 \mathrm{~b}$ ); and if too small, a mushroom-shaped excrescence forms on its tip, and the lamp burns unsteadily (Fig. $30 \mathrm{c}$ ). In the former case the resistance in series with the lamp requires increasing, so that the current is decreased in amount, while in the latter case the resistance should be decreased, a corresponding increase of current resulting. The distance between the carbons must also be modified-either lengthened or shortened-to conform to the altered conditions.

The distance between the two carbon points is of primary importance, and on the careful regulation of this distance and its constant maintenance depends the constancy of the source of light. In the case of a continuous current, the positive pole is the light-producer; the negative pole only produces half as much light as the other, and, as already explained, this should not be utilised. The positive carbon is also consumed at a rate that is approximately double the rate of consumption of the negative. To obviate the difficulty due to this difference of consumption, the sectional area of the positive carbon is made twice that of the negative one, so that the reduction of their lengths is practically constant. In the case of an alternating current, which is for many reasons practically useless for microscopic work, the rate of consumption of the two carbons is equal. In this case too, an additional difficulty is that the two carbon poles are equally incandescent and, consequently, only one-half of the light produced can be used. Further, any method of automatic regulation is much less certain than with continuous currents, and even in hand-feed lamps, the are is very liable to acquire a periodic rotation round the edges of the craters, which is fatal to its use for photo-micrography.

Of all electrical illuminants the arc light has the highest efficiency, and it has the advantage that its luminous point is quite regular, so that the image of the light-source in the objectplane of the microscope can be easily obtained, giving an evenly illuminated field. A convenient current consumption is from seven to ten amperes if a light of moderate intensity only is required, but by increasing the current consumption to twenty-five or thirty amperes, a light-source of sufficient intensity for the highest power work is obtained. It should be understood that increase of current consumption does not 


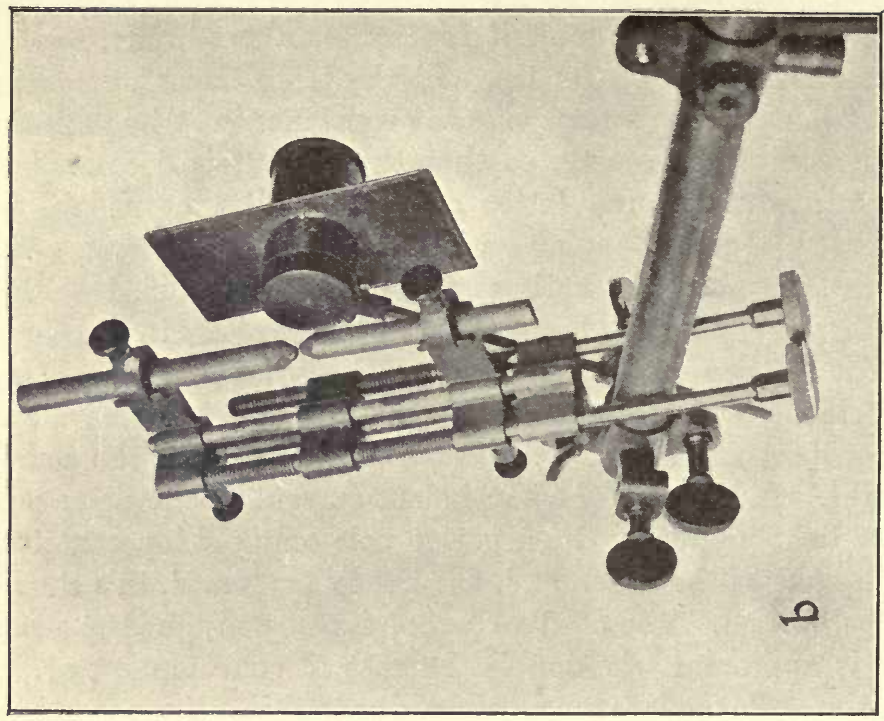

䓪 
result in any higher degree of incandescence of the luminous crater. The condition of the carbon is already such that it is at the point of volatilisation, so that increase of current cannot raise it to a higher state of incandescence. But, the greater the current, the higher the efficiency of the are light from the point of view of total light production, that is, increase of current results in more than a proportionate increase in the area of the incandescent crater, and consequently the amount of light produced is greater.

By far the simplest and most certain method of adjusting the length of the are and keeping it constant both in position and condition is the use of a simple hand-feed arrangement. As the result of experiments carried out in collaboration with Dr. T. A. B. Carver the writer adopted and has since used constantly a simple hand-feed method of arc regulation, and in spite of the improvements made in automatic lamps some such device is to be preferred.

Figs. $31 a$ and $b$ illustrate the method of adjustment. The carbons are held in V-clamps sliding upon two vertical rods, and their relative positions can be varied by turning the right- and left-handed screw which is controlled by a milled head. The position of the whole are, that is of both the carbons, can be varied without altering the length of are by turning the serew $\mathrm{B}$, as this engages in the bracket $\mathrm{E}$, causing it to move up and down the two vertical rods C and D. The lamp may be held by any convenient method, but is shown mounted on a boss sliding on a tube. Its inclination can be altered by loosening the screw $\mathrm{F}$, which allows any required inclination to be obtained. As the figures show, the lamp can be carried either on a vertical or on a horizontal rod, so that it is easily adaptable to any conditions under which it may be required to work. With this arrangement it is quite simple to feed the are so that it maintains its truly central position in relation to the microscope and constant distance between the carbons themselves.

Any other simple and exact method of feeding the carbons may be adopted, and Fig. 32 shows another form of mechanism. Here the two clamps holding the carbons are supported in a centre casting, and are actuated by an ordinary rack-andpinion motion, this latter being controlled by the milled head B. 
The vertical adjustment of the arc is controlled by screw D and lateral adjustment by the milled head $\mathrm{C}$. The milled head A allows of lateral movement of the positive carbon-holder so that the positive crater is caused to form towards the front of the lamp. It is essential. in selecting any of these hand-feed arc lamps, to see that whatever arrangement is decided on, there is a complete absence of shake, and no unsteadiness of movement or displacement other than in the direction desired, when either of the adjustments are brought into play.

The particular means by which the carbons are moved in

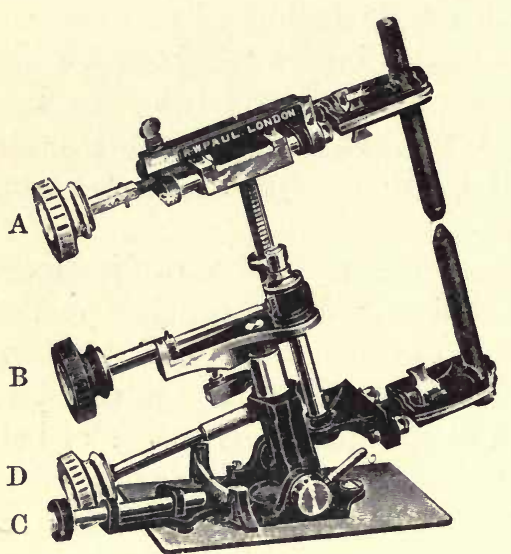

FIG. 32.-Hand-feed Electric-arc Lamp. relation to one another is not of great importance, provided that it is such that both carbons are moving towards each other at an equal rate, and that no lateral movement occurs. It is, however, essential that there should be a means of moving the carbons simultaneously, so that without alteration in their relative position, the arc is itself moved as a whole. This, however, is not an easy matter, unless there be some means of observing the alteration in the length of the arc or in its position. This can be easily provided for by arranging what is in fact a simple pin-hole camera at the side of the are, as shown in Fig. 31. A pin-hole at the end of the tube nearest the light throws an image of the two carbon points on to the small ground-glass screen shown at the other end of the tube. Consequently, any alteration in the length of the arc or in the position of the positive crater can be immediately seen by direct observation on the ground-glass screen. It is only necessary to provide on it suitable reference cross-lines to enable the observer to at once adjust the position of his source of light, and to maintain it in an absolutely constant condition and 
position. This arrangement is perhaps the simplest and most efficient that can be obtained for photo-micrographic work, as it is obviously only necessary, once and for all, to set the pin-hole camera in the position that coincides with the optical axis of the microscope. On starting the are, therefore, no further adjustment is necessary with the microscope itself, the subsequent observations to be made being only those through the pin-hole camera. It will be found in practice that any adjustment of the arc, whether for position or length, is only necessary at intervals of one to two minutes, so that in any ordinary length of exposure an adjustment made before the exposure is commenced will be all that is necessary to ensure the arc remaining in its proper condition for a sufficient length of time. When the lamp is started no attempt should be made to take a photograph with it for a minute or two, as probably owing to the fact that there is a considerable amount of air occluded in the carbons, the light does not become steady until it has been driven off. The pin-hole camera will always clearly indicate when the light is ready for use.

It does not come within the province of this work to discuss the construction of automatic arc-lamps in detail, so that only two well-known types will be referred to.

In the lamp supplied by Messrs. Carl Zeiss, the carbons are automatically fed towards one another, but there is a contrivance by which the positive carbon is kept in an approximately constant position, and by adjusting the screw which projects at the back of the body the position of the positive crater may be varied; the automatic arrangement then ensures it being kept in its correct position. There are also two screws at the side of the body of the lamp by which lateral adjustment is made.

In the automatic lamp supplied by Messrs. Leitz, the method is adopted of placing the carbons at right angles to one another ; the object is to project the light from the positive carbon directly towards the microscope, the negative carbon being sufficiently low down not to intercept any of the required light.

This arrangement requires that the positive carbon should be as small as possible in diameter for the current used, so that 
the crater occupies a large portion of the end of the carbon. The tendency is for the are to take the shortest course across the gap between the two carbons, and consequently it may happen that more light is projected behind than in the direction of the microscope. It has the decided advantage that, after the necessary preliminary adjustment, the positive crater always remains central, since the consumption of the carbons takes place in the direction of the optical axis.

It must be clearly recognised that any form of automatic regulation, unless it is of the very best construction, is worse than useless. Evidently in the hand-feed arrangement there is no mechanism to get out of order, but in the automatic the whole efficiency of the apparatus depends on its at once responding to small changes in the current flowing through the lamp.

Of necessity an automatic lamp is intermittent in action, and its mechanism is only brought into play as the result of some lengthening of the arc and consequent alteration in the amount of current; how much change is required to set the carbons in motion depends entirely on its sensitiveness. It is therefore difficult to ensure the constancy and accuracy of movement which can be obtained by a simple hand-feed apparatus.

An automatic lamp generally takes some ten or fifteen minutes for the carbons to burn themselves into shape, and even that time may be exceeded if the current that is being used is greater or less than the lamp is designed for.

It will be advisable to give some brief instructions as to the method of connecting up an arc lamp to the source of electrical supply. Unless there is already extra heavy wiring sufficiently large to carry the current, it will be absolutely essential to have special wires brought, preferably from the distributing switch-board, to the point where the lamp is to be run. These wires will require to be considerably thicker than those used for ordinary lighting purposes, and should have fuses, or what are known technically as ' cut-outs,' exactly suited to the current proposed to be used.

Fig. 33 indicates the method of connecting up the lamp. The wires are brought from the terminals on the board shown at $\mathrm{A}$, respectively positive and negative, as marked. They then pass successively to the safety fuses, and from thence to 
$\mathrm{B}$, a double pole-switch for making and breaking the circuit. One wire is continued from either terminal-it matters not which-to one end of the resistance coil C. The other terminal of the resistance is then connected up directly on to the proper terminal of the lamp. The other wire from the doublepole switch is then connected directly from the switch through to the other terminal of the lamp in the same way, except that it does not connect to the resistance.

When the lamp is to be started, then if it be automatic,

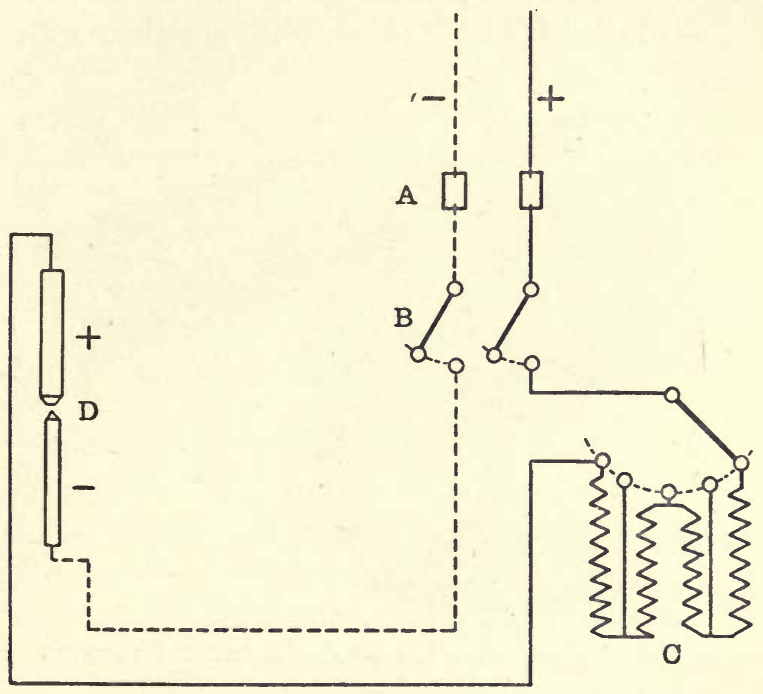

FIG. 33.-Method of connecting up Electric-arc Lamp.

it is only necessary to switch on the current, and the are is at once made. With the hand-feed lamp, after the current is switched on, the two carbons have to be brought together momentarily and drawn apart, as already explained, when the lamp will immediately light.

If desired, an ammeter and voltmeter can be connected up in the circuit. The ammeter is arranged so that the whole of the current passes through it in the same way as through the resistance, i.e. ' in series.' The voltmeter must, however, be connected up as a shunt-circuit, so that it indicates the difference of potential on the terminals of the lamp. These 
electrical instruments are by no means necessary, although at times they are a convenience; but if all the necessary arrangements are made by a reliable and competent electrician, as they most unquestionably should be, there will be little need for their use.

A convenient form of hand-feed arc lamp for use with small currents is that recently introduced by Messrs. Leitz of Wetzlar (Fig. 34). In it the positive and negative carbons are at right angles to one another; and, although this arrangement cannot be entirely justified on theoretical grounds, it must be admitted that in practice, with a lamp

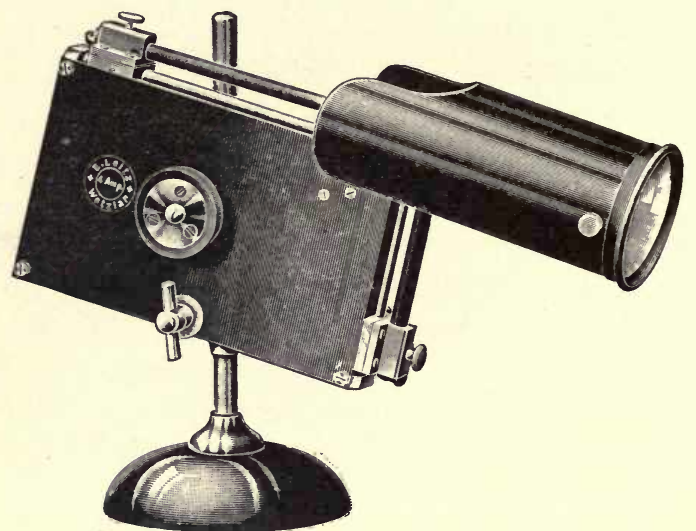

Fic. 34.-Hand-feed Electric-arc Lamp for a Current of 4 amperes. (E. Leitz.)

using small carbons, it works extremely well. The tendency with this type of lamp is for the are to form in a position which does not allow of the maximum light being projected forward; a great amount is then emitted towards the back, and so is, for practical purposes, lost. It may be that on very extreme tests as to efficiency this small lamp of Messrs. Leitz would not be better than one in which the carbons are approximately vertical, but in actual practical use there is little or nothing to be urged against it. The difficulty usually found in the type with carbons at right angles, due to the lower negative carbon coming in the way of and obstructing the light emitted by the positive carbon, is partially overcome by using a positive carbon which is somewhat smaller 
than the negative. To compensate for the more rapid consumption of the positive carbon, which would of course occur on a continuous-current circuit, the positive feed is more rapid than the negative. As the positive carbon is smaller than the negative and is kept as small as possible for the current used, the size of the luminous crater is, in relation to the total sectional area of the carbon, fairly large ; the crater, in fact, occupies nearly the whole of the end of the carbon itself.

The lamp is provided with a condensing-lens arranged so that it projects approximately a parallel beam. It also has an adjustment for altering its inclination, so that for visual work, and particularly for use with the modern dark-ground illuminators, it may be inclined at any suitable angle. The feeding arrangements for the carbons are simple and certain in action, and the feed is sufficiently exact to ensure that the carbons shall move in perfect alignment. The lamp is designed for a current of four amperes, but it will work with a considerably greater or smaller current consumption than this. It must of course be used with a suitable resistance in series to somewhat suit the particular circuit on which it is intended for use.

The difficulty inherent in all illuminants, particularly electrical ones of small area and irregular shape, is so to arrange them that the image of the source of light will cover the object under observation. With either carbon or metallic filament electric lamps, or even with the Nernst electric lamp, the filament is of considerable length, but is very narrow; the consequence is that if a direct image of the source of light is projected it is impossible to fill even a high-power objective with light. A common way of overcoming this is either to enclose the source of light in a ground-glass globe, or to interpose between it and the sub-stage condenser a piece of ground-glass. The objection to this method is that too often the irregular surface of the ground-glass is itself projected into the field of view, and produces an appearance which for visual work is disconcerting, and for photographic purposes is absolutely impossible. Mr. J. W. Gordon has suggested the application of a speculum which overcomes the difficulty and enables a light-source of small area or irregular shape to be used. It consists essentially of a piece of glass rod, one end of which is polished and the other cut to a plain surface and finely ground ; 
this is interposed between the source of light and the sub-stage condenser of the microscope, the ground-glass end being towards the radiant. The rod may conveniently be made of about half an inch or less in diameter, and of a length from four to six inches. It may be placed quite close to the radiant, since from its form it is particularly tolerant of any considerable rise of temperature, and the glass is sufficiently conducting to prevent risk of fracture or of fusion. By varying the distance between the ground-glass end of the rod and the radiant, any desired variation in the intensity of illumination may be obtained. Evidently, the intensity of illumination will vary inversely as the square of the distance between the filament and the ground-glass surface. The light which in this way is emitted from the ground-glass surface is transmitted by total internal reflection almost in its entirety along the length of the glass rod, with the result that the illumination is even and uniform at whatever distance the speculum is from the radiant. In effect therefore the speculum acts as a source of light, which has none of the disadvantages of the original illuminant. The apparatus in a convenient and perfected form may be obtained from Messrs. Beck of Cornhill, E.C.

For visual purposes this appliance is of very considerable use; but for photographic work, where usually some method of broadening the image of the source of light by using collecting lenses between the radiant and the sub-stage condenser is employed, its use is not, perhaps, quite so necessary. But it may be adopted with advantage where carbon or metallic filament electric lamps are the only sources of light available.

Sunlight.-Direct sunlight may be necessary for some classes of work, but with it the use of the heliostat is advisable, if not absolutely necessary. Any type in which the clockwork movement is accurately and carefully made can be adopted. It is necessary for it to be adjusted for the particular latitude in which the work is being carried out. In this country it can hardly be regarded as a satisfactory method of illumination and will probably rarely be adopted. It introduces considerable difficulty in estimating exposures owing to the variability of the actinic value of sunlight. It must be admitted, however, that in the early days of photo-micrography it was necessary, and good results were obtained; but the photographic plates 
then available were not to be compared in sensitiveness to those of to-day and the optical appliances were relatively crude. The lenses were usually of small aperture, not so well polished, and made of glass of less transparency than at present.

A heliostat designed by Dr. G. Johnston Stoney, which is particularly suited to the requirements of the physicist and photo-micrographer, is made by Messrs. W. Watson \& Sons of High Holborn, and is throughout of first-rate construction.

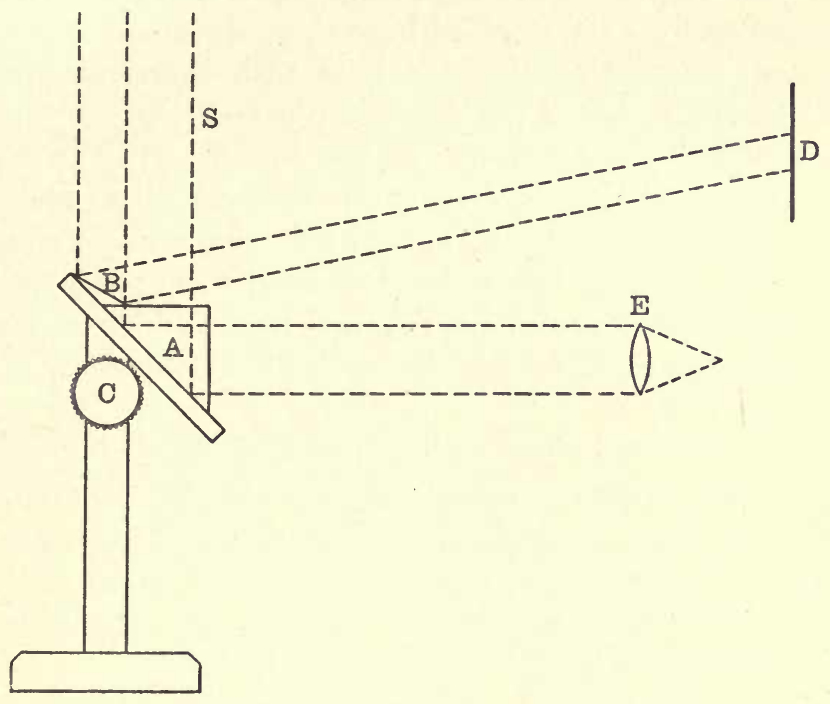

Fir. 35.-Simple Form of Heliostat.

Fine adjustment for setting the instrument in its precise position is afforded by means of rackwork and pinion and tangent screw.

A possible substitute for the heliostat where its cost is prohibitive, or where for other reasons it is unobtainable, is the arrangement suggested in the diagram, Fig. 35. Sunlight is allowed to enter the room in which the work is being done, in the direction as shown by the dotted lines S. The rays will, of course, be parallel. They strike either on a mirror, which should have a silvered surface, or preferably on a reflecting prism $\mathrm{A}$, and are thence reflected in the direction $\mathrm{E}$, the latter representing the sub-stage optical system of the microscope. 
If a small subsidiary mirror $B$ be fastened on one edge of the prism, roughly in the position as shown, but in such a way that it does not materially cut off the incident ray, then another narrow beam of light is reflected in the direction $D$. It is evident that the small mirror is so placed that it does not reflect a beam at right angles to the incident beam. The reflecting system should be supported on any suitable stand, and should be capable of adjustment about an axis situated at the centre of the reflecting surface of the prism. This movement should be controlled by a large milled head $\mathrm{C}$, or the prism should be supported on a ball-and-socket joint with universal motion. It will be found that if the subsidiary reflected ray is thrown on to any white surface, such as the ceiling or the wall of a room, and its position indicated by reference cross-lines when the main beam of light is in the optical axis of the microscope, it is then not difficult, by carefully rotating the milled head $\mathrm{C}$ or by moving the prism supports and by watching the spot $\mathrm{D}$, to keep the rays of light always truly in the axis of the microscope.

Some similar arrangement has been used for photographing stars, and the writer has successfully worked with an arc lamp in which this idea was utilised. It is obviously of the nature of a makeshift arrangement, but yet it is one of those methods that in the hands of a careful worker will give results in no way inferior to the most expensive of heliostats. 


\section{CHAPTER VI}

\section{THE PHOTO-MICROGRAPHIC CAMERA}

Simple Forms of Vertical Camera.-Having considered the optical part of the apparatus in the earlier chapters, reference must now be made to that portion which is more particularly of a photographic character. Essentially this consists only of an ordinary photographic camera, and it will probably happen that the majority of workers who are starting from the beginning will at least make some attempt to obtain photo-micrographs with such a camera used in conjunction with a suitable microscope. Under these conditions, all that is required is that the microscope shall be set on some form of firm table or bench, and that the camera, of course with the ordinary photographic lens removed, shall be so placed that the microscopic image is projected directly on to the ground-glass focussing-screen.

Under such circumstances the only points that require careful attention are, firstly, that the camera should be placed with the centre of the focussing-screen in alignment with the optical centre of the microscope, and, secondly, that the focussing-screen should be exactly at right angles to the optical axis.

It is evident, however, that such an arrangement is open to considerable objections, since, although it may by its aid be quite possible to obtain good photographs when using low powers, it becomes a matter of considerable difficulty if the magnification exceeds some twenty or thirty diameters; for there is no arrangement to ensure that the relative position of the microscope and camera is not altered during the process of focussing and exposing.

Nearly all who take up this work at some time or another 
make an effort to build or to design and have built an apparatus according to their own ideas, and the writer is strongly of the opinion that such an arrangement, crude though it may be, usually gives the best results in the hands of an intelligent worker. Each one will have ideas which as the result of experiment they would like to carry into practice, and an apparatus built up as the result of practical experiment is usually most satisfactory.

The designs for an apparatus may be divided very broadly

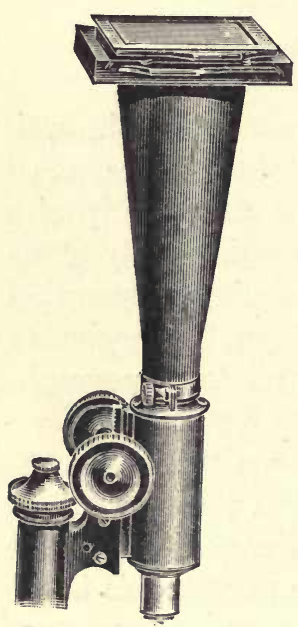

Fig. 36.-Simple Form of Photo-micrographic Camera. (J. Swift \& Son.) into two well-defined types. In one arrangement, and probably the one which is most generally used and has the most to recommend it, the microscope and camera are supported on one very stiff and solid base, so that no alteration of either part in relation to the other can take place. In the other type the microscope and camera are supported and treated as two separate pieces of apparatus, the idea in this design being that where any outside influence tends to disturb the position of either of the components, it would then only affect one of them. For instance, if in the course of focussing the image on the screen of the camera, in inserting the dark-slide or in withdrawing the shutter, some alteration takes place in the position of the camera, the microscope and its adjustments will not be affected. It is claimed for this method that the greatest evil would result from any movement of the microscope-and such is unquestionably the case-therefore that the microscope should as far as possible be isolated and so arranged that it is free from such disturbing influences. One of the greatest troubles usually met with where the work is carried out in an ordinary room or laboratory is vibration or tremor. Well-defined shaking of the apparatus is rarely a factor, as any worker would usually take reasonable precautions against any dislocation of the apparatus, but it is the ordinary tremors that are the most difficult to provide against. It would appear 
that the type of apparatus in which everything is supported on, and, if thought advisable, clamped down to, a solid and heavy base, would be the better type ; in such case any vibration affecting the apparatus would affect the whole of it equally, and no disturbance of the components in relation to each other would result.

Where a simple type of apparatus is for any reason decided on, and where no special bench or supporting-table can be entirely devoted to the work, it is probable that what is known as a 'vertical' camera would be the best type to adopt. In this the microscope is kept in a vertical position and the light therefore has to be reflected through the sub-stage condenser by the mirror, as under ordinary conditions in visual work. The camera is supported over the microscope so that the image is thrown on to the focussing-screen, which therefore lies in a horizontal position. This type of apparatus necessarily has limitations ; the length of camera is usually restricted to from 18 to 24 inches, and consequently, unless high-power projection oculars are used, high magnifcations are not easily obtained.

For the purpose, however, of recording the results of researches where critical definition is not of such importance, this apparatus is extremely useful. One of the simplest forms is that shown in Fig. 36,

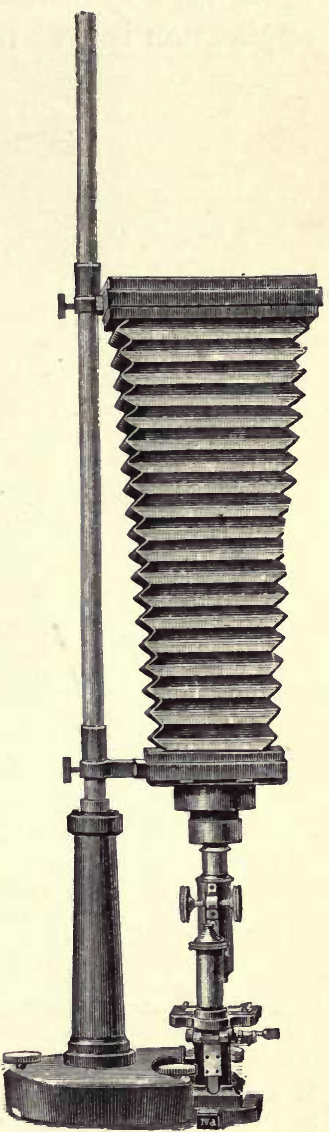

Fro. 37.-Vertical Camera. (Zeiss.) made by Messrs. J. Swift \& Son. It consists simply of an aluminium cone which fits over the upper end of the body-tube of the microscope. It is not more than about 12 inches in length, and supports on its upper end a frame which carries the ground-glass focussing-screen and the plate-holder. For ordinary laboratory work it is a very useful piece of apparatus ; 
but its use requires more than ordinary care, owing to the fact that in inserting the dark-slide, or in withdrawing the shutter of the latter, there is considerable liability of altering the focus of the microscope.

A type of vertical camera which admits of rather wider application is that made by Zeiss, Leitz, and others, in which

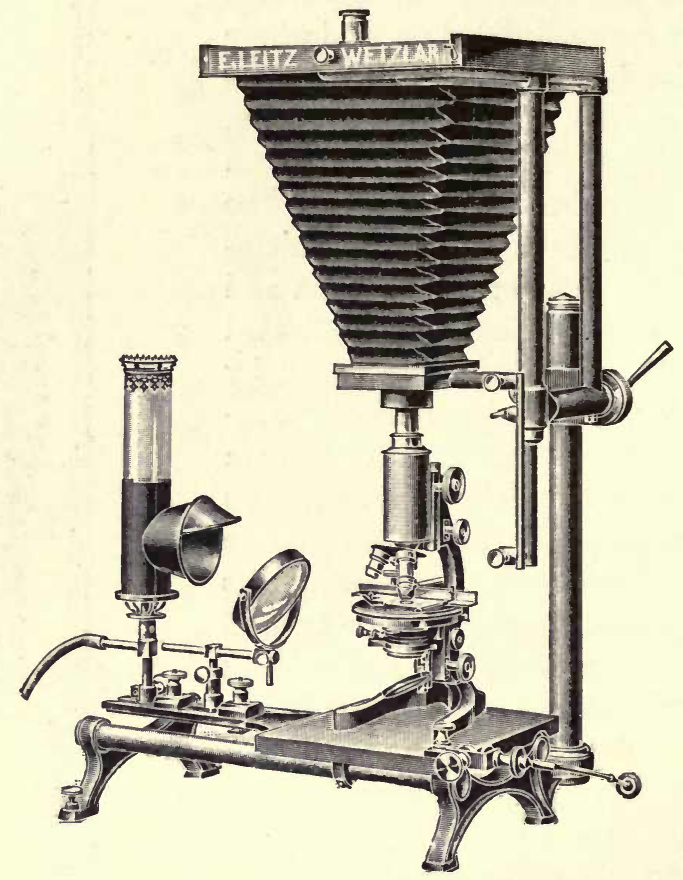

FIG. 38 a.-Combined Horizontal and Vertical Camera. (E. Leitz.)

the camera is supported separately from the microscope (Fig. 37). The arrangement is generally one in which a stiff steel rod is supported on a tripod foot ; the camera, both at the lower end near the microscope and also at the frame which carries the focussing-screen, is fixed by clamps to the rod and is capable of adjustment in a vertical direction. The microscope stands on the table as before, so that there is no rigid connection between the microscope and the camera. This type is a very convenient one, since the camera can be bodily removed, or can be swung round on the supporting-rod when observations have to 
be made, and replaced again over the microscope at will. It is, however, open to the rather serious objection that the support of the focussing-screen or the dark-slide is at the end farthest away from the tripod, and we have thus very much the same conditions which obtain when a metal bar is rigidly supported at one endnamely, that the rod is specially subject to any vibration. Such vibration would result in the greatest movement of the upper end of the camera, and consequently would have the very worst effects on the definition of the photographs.

A further development of the same type is one in which the camera is still carried on a vertical rod, but its base is made so that the microscope can be placed on it (Fig. 38a). There is also often an additional arrange-

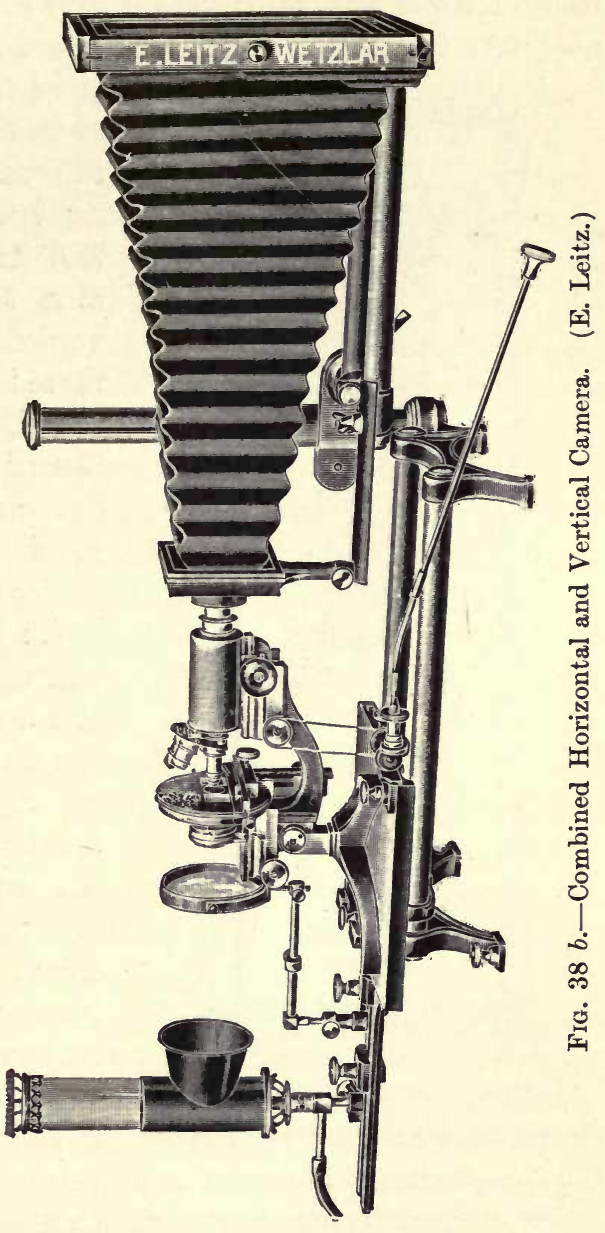
ment by which the

camera can be used in either a vertical or a horizontal position (Fig. 38b) ; further, it is then supported on a pivot that the camera when used in either position can be easily swung out of the way for the purpose of making any observation. Although this type has a firm base, carrying both camera and microscope, and although the component parts of the apparatus 
form one unit, yet it is open to practically the same objections as the previous one, the camera itself being rather liable to vibration owing to its method of support. While it is a very useful form of apparatus for ordinary laboratory work, it should not be used in preference to a more stable horizontal form,

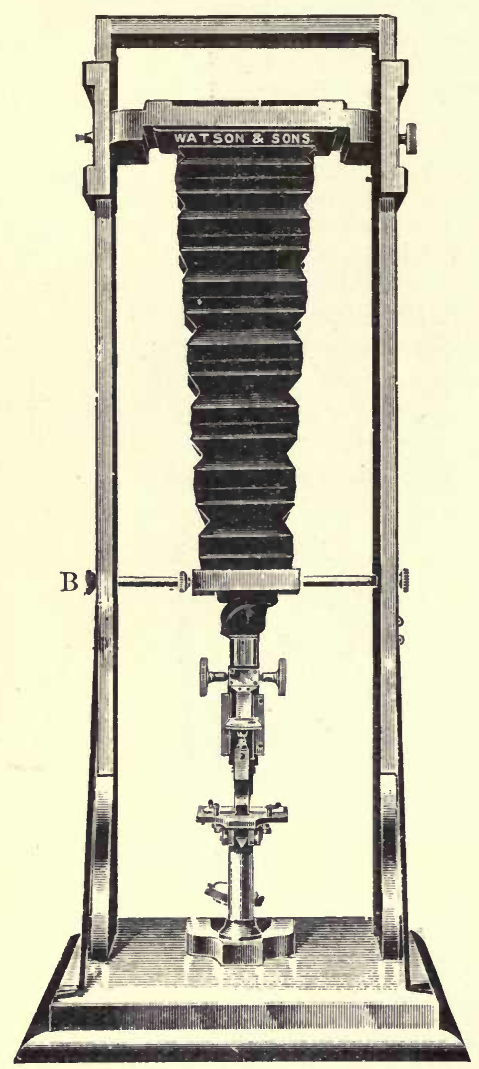

FIG. 39.-Combined Horizontal and Vertical Camera. (W. Watson \& Sons.) since it is designed chiefly for obtaining photographic records of researches.

Where a combined vertical and horizontal camera is for any reason the most convenient, then one in which there is on either side a support for the frame carrying the focussing-screen is preferable; the apparatus is then not so liable to respond to extraneous vibration, and is much less liable to movement while making any adjustment. Fig. 39 illustrates a convenient and efficient form of this arrangement. The baseboard is stout, and the uprights on either side are rendered firmer by two struts that come from the baseboard to a point approximately halfway up the sidestays. The focussing-screen is adjustable in grooves, and can be raised or lowered and clamped in any position within the range of the apparatus. A shutter is provided in the position B, although this addition is for most purposes superfluous. Better results would probably be obtained if this shutter were not included, since any arrangement for cutting off the light should be independent of either camera or microscope. The bellows of the camera give an extension of 20 inches, which is ample for any 
work that would be possible with such an apparatus. As this camera is reasonable in price, and substantially made, it is one that may be recommended where an elaborate outfit is not desired.

Some form of vertical apparatus is, however, an absolute necessity at some time or another to the photo-micrographer, and the type adopted by the writer is that known as Van Heurck's vertical camera, made by Messrs. W. Watson \& Sons of High Holborn (Fig. 40). This apparatus is particularly rigid, and, as the illustration shows, consists essentially of an elongated box supported on either side by strong supports, and carrying the focussing-screen and dark-slide at its upper end. On the side of the box near to the observer is a door which, while it can be opened for making any adjustment to the microscope, is perfectly light-tight when closed and ready for taking a photograph. The chief objection to its use is that there is no method of altering the distance between the microscope and the focussingscreen, so that any variation of magnification has to be entirely effected by a suitable combination of objective and ocular. In practical work, however, this is a comparatively small matter and one that a little ingenuity will overcome. The apparatus is very convenient for photographing objects that may lie in fluid, or in cases where for any other reason the microscope cannot be inclined.

The dark-ground illumination methods that have lately been re-introduced have rendered the photographing of such objects as living bacteria quite within the bounds of ordinary work, and the apparatus is eminently suited for this purpose. The table on which the microscope is supported should be very strong and stiff, and it is essential to see that when the camera is placed over the microscope it is perfectly steady and is standing on a level surface. The apparatus as shown is supported on four points, and mechanically this is unsound. The writer's modified apparatus is made with one strong leg on either side, and a third one on the opposite side to that on which the observation-door opens. The worker is therefore independent of any inequalities of either table or bench, as the apparatus, being supported on three points, will in any case stand quite firmly, while being as stable as when supported on four points. 


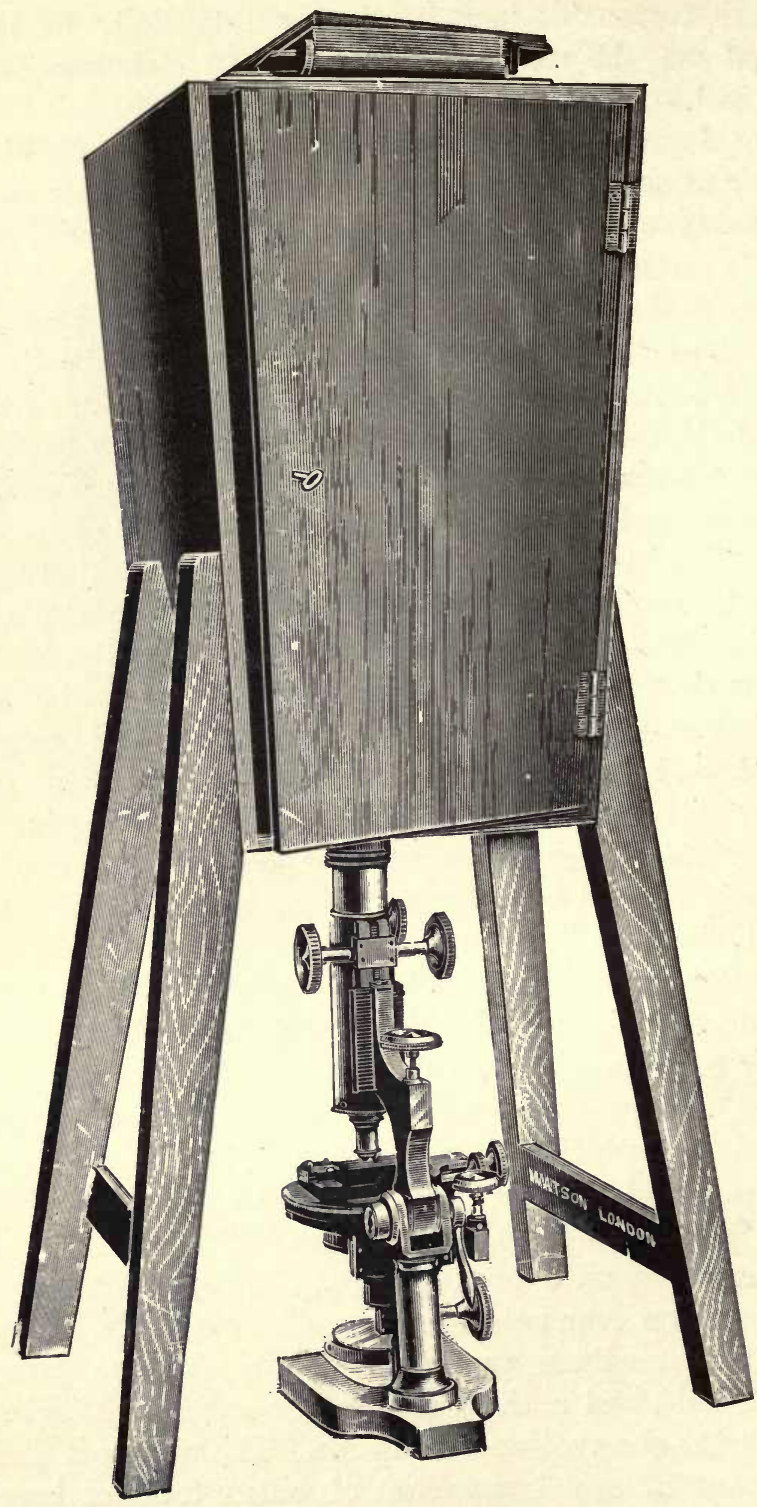

Fig. 40.-Van Heurck's Vertical Camera. (W. Wåtson \& Sons.) 
A vertical apparatus that presents some novel features is that designed by Mr. J. W. Gordon, and made by Messrs. Beck of Cornhill. It is so arranged that the object having once been focussed in the microscope in the ordinary way, the camera only has to be placed over it and the photograph taken-no readjustment of the microscope in fact being necessary to ensure a sharp image on the photographic plate. It consists of a tube, about six inches long, which is placed over the microscope eyepiece when a photograph is required. At the upper end of this tube a small photographic plate $1 \frac{5}{8}$ inches square is held by means of a cap; between this and the eye-piece is a projectionlens which focusses the image upon the plate, and a small shutter for making the exposure is placed in the tube. If the observer's sight is normal, the photograph will be sharp when the microscope is in its proper visual focus; but, as almost everyone has some slight errors of vision, it is found desirable to supply a duplicate tube with a focussing eye-piece of high power which is first placed in position on the instrument and focussed, and is then replaced by the camera. This apparatus therefore becomes an essential part of the microscope, and since it is very small and supported at such a short distance from the optical system, it is less subject to the effect of vibration than some other arrangements; but still, in any case, it should be placed so that it is as free from the influence of tremor as possible.

Where a vertical camera is required simply as an adjunct to a more complete and elaborate horizontal one, an improvised arrangement such as Fig. 41 is as good as many at present on the market. Anyone with a slight knowledge of carpentry can make such an arrangement, or it can be constructed at a very small cost by a mechanic. It consists simply of a baseboard about twelve to fourteen inches square, and one inch thick; underneath this should be screwed two cross bracing pieces of the same thickness in a direction across the grain of the wood, so that the chance of warping is reduced to a.minimum. Two other lengths of wood of the same thickness, each about three inches broad, should be fastened together in T-form, as shown in the illustration, and these should be supported at right angles to the baseboard and firmly screwed down to it. On the flat side of this vertical piece, another thinner piece of wood should be placed in which is a narrow slot about three-eighths of an inch broad; any 


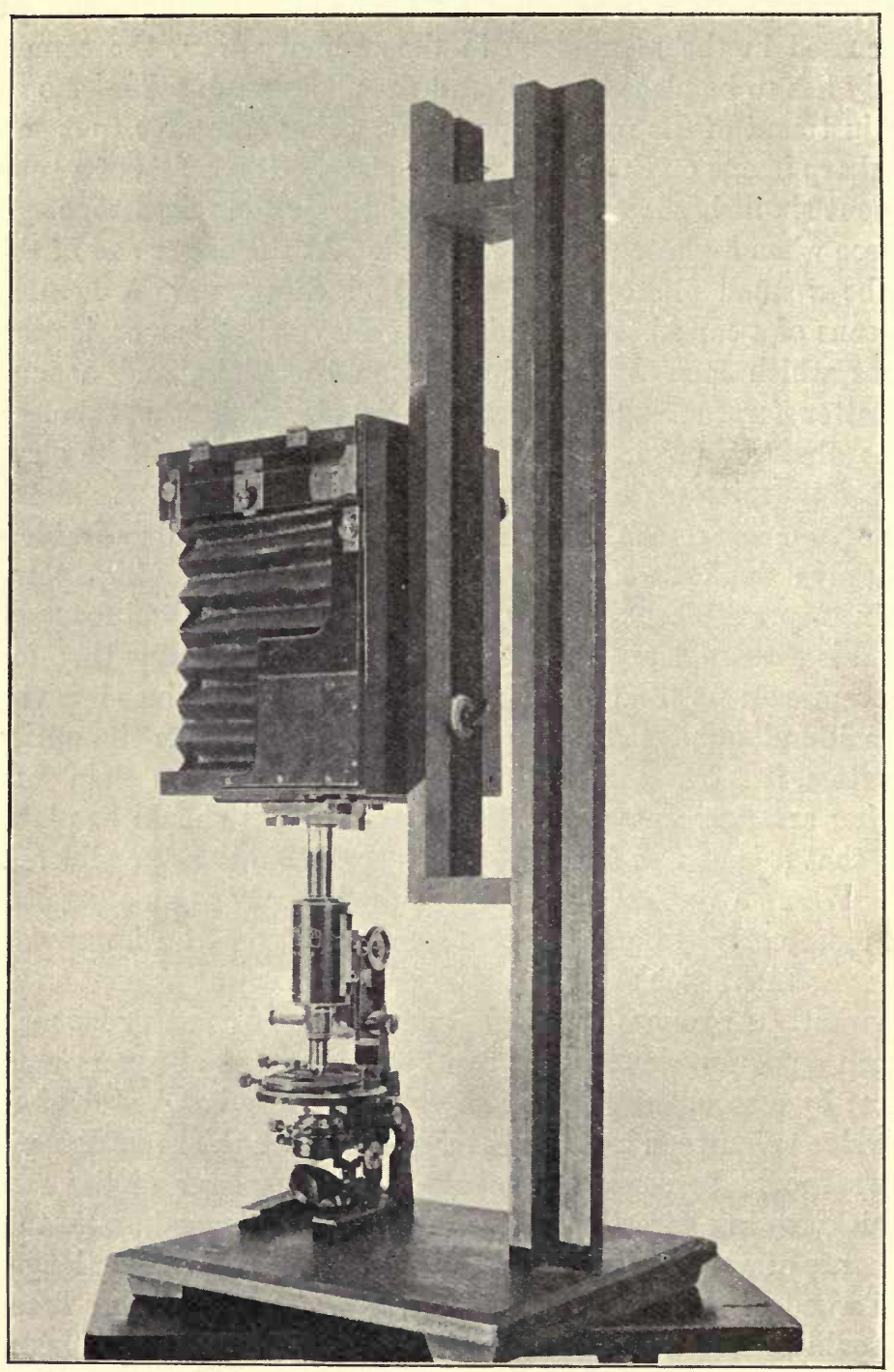

Frg. 41.-Ordinary Camera used as Vertical Camera. 
long-extension ordinary photographic camera can then be clamped on to this with a suitable clamping-screw, and can be raised or lowered into any required position. The microscope is placed underneath the camera, the image being projected upwards on to the ground-glass focussing-screen. Such an arrangement admits too of easily photographing large objects that do not require a microscope at all, and yet that readily come within the purview of a photo-micrographer. There are again many objects, such as bacteriological tubes and plate-cultures, which at times require to be photographed, and which may be much more conveniently dealt with if they are lying flat down than if they have to be supported in front of a camera in a

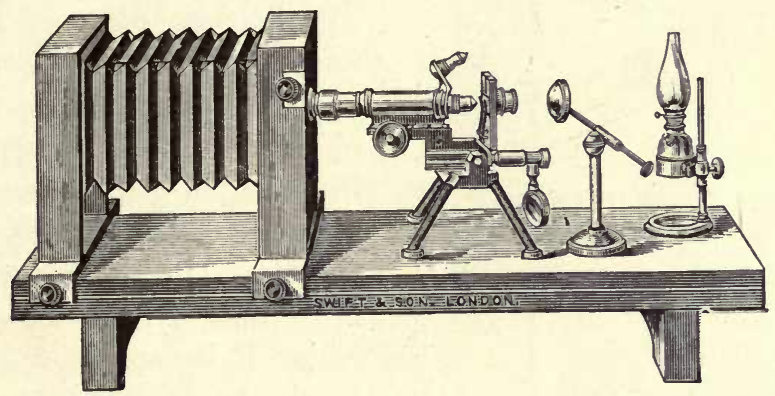

FIG. 42.-Simple form of Horizontal Camera. (J. Swift \& Son.)

horizontal position. This simple support enables most objects of this nature to be easily dealt with, and it is also available for low- or moderate-power photo-micrographic work when required.

Horizontal Cameras, - Of the horizontal types of apparatus, Fig. 42 is an illustration of the simplest possible form. Here an ordinary plano-convex collecting-lens, microscope, and camera, are simply placed on a wood base in their correct relative positions ; no arrangement is made for ensuring that they are in correct alignment or for enabling them to be replaced should either of the parts for any reason be removed. Obviously this arrangement is of a very simple description and has little to recommend it for other than the most elementary work. Such an apparatus would usually be improvised by the worker out of an ordinary long-extension 
photographic camera. It may, however, be improved with little trouble or cost. Guides should be placed on the base-

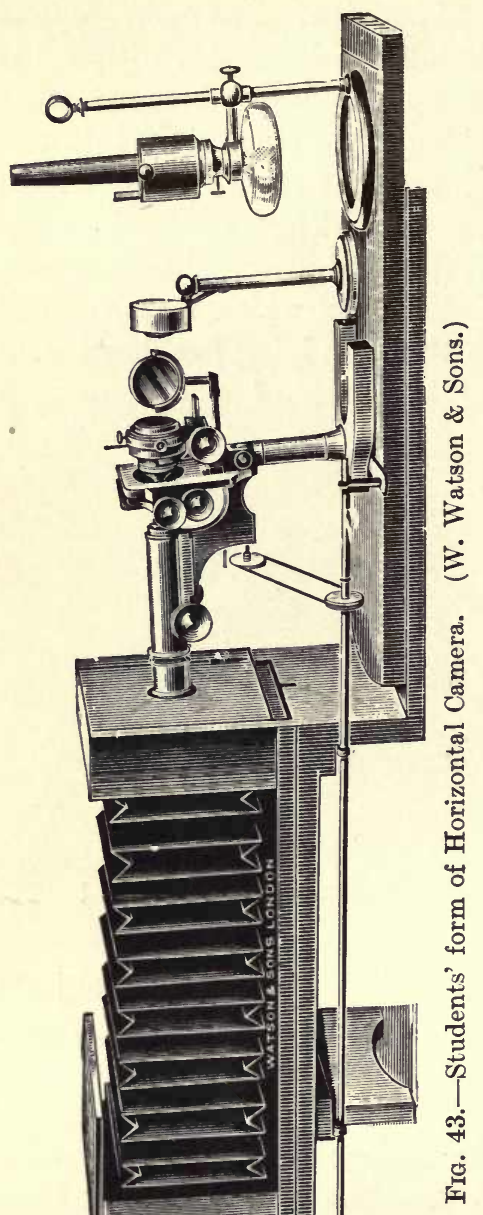
board for the microscope to drop between, so that it could always be replaced in its proper position, and the same might be done with the lamp and the bull's-eye condenser; beyond this, little more can be done with so simple an apparatus. The camera has in this case to be kept very short, so that an observer can reach the fine- or coarse-adjustment of the microscope while observing the image on the ground-glass focussingscreen. The possibilities, therefore, of the apparatus are limited, and the work which it is capable of doing would be confined to that with low-power lenses.

The next step towards greater elaboration should consist of an elongation of the baseboard, rendering it possible to use a camera with greater bellows extension. When this is done jt becomes necessary to have an arrangement for controlling the fine-adjustment of the microscope while the observer is watching the image on the focussing-screen. There are many ways of doing, this, and a simple one is that shown in the illustration (Fig. 43). A long metal rod is supported on the wooden base and has 
a large milled head at its end near the focussing-screen. At the other end is a grooved pulley, and between this and the fine-adjustment of the microscope a connection is made, usually consisting of a light cord which passes from one to the other. This is preferably a silk thread well rubbed with bees'-wax so that it is not liable to slip on either pulley; in any case it should be taken from one to the other so that there is no strain; in fact, it will be found that with waxed silk a very loose connection will quite efficiently turn the fine-adjustment screw, and that it will respond immediately to changes of direction. Some inexpensive additions to this type of apparatus will increase its efficiency very greatly. It is convenient to be able to make an observation in the microscope, or to alter any of the adjustments while observing the image directly through the instrument. This may be effected in two ways: either by carrying the microscope and the illuminant on a separate board or support, as shown, and so arranging it that it can be swung out on a central pivot as an axis; or, as in the types of apparatus devised by Messrs. Zeiss, the microscope itself may be on a sliding base so that it can be moved laterally in or out of the axis of the whole apparatus. The former method is preferable, as there is then no alteration in the relative position of the optical system and the illuminant: when the turntable is swung round it allows an observer easily and conveniently to adjust the light, or any of the sub-stage illuminating apparatus, without having to watch the effect of any such alteration on the focussing-screen of the camern.

The apparatus (Fig. 43) embodies both the arrangement for focussing from a distance, and also a simple form of turntable carrying the microscope, and, as it is reasonably stiffly built, it would answer for moderately high-power work. If, however, an apparatus is required that will enable any class of work to be attempted, then still further elaboration is advisable.

Figs. $44 a$ and $b$ illustrate an apparatus made by Messrs. Ross of New Bond Street, London, which embodies all the requisite adjustments. It is designed for use both for transmitted and incident light, so that it is available for metallurgical or similar work in addition to the photographing 
of any transparent objects. The whole apparatus is supported on a stiff base, the camera having a considerable extension and being sufficient for high-power work. The optical system and the illuminant are carried on a triangular metal bar, so that any part of the apparatus may be adjusted in the direction of the axis of the microscope, or can be removed and replaced at will without alteration of position and without the necessity of re-adjustment. The base of the camera is in two parts, the one telescoping into the other, and it is so arranged that when only a short camera is in use there is practically no extension of the base to interfere with an observer, enabling him the more easily to manipulate the apparatus from the rear. The bar carrying the illuminating system is attached by means of a clamping-screw, and takes a bearing on a plate sliding along the top of the base. The other end is supported on a foot with ball castors, which rests on the table or bench supporting the whole apparatus and is provided with adjustment to regulate the height. The bar can be arranged so that its near end is in close proximity to the plane of the microscope stage, or it may be set back so that it is as much as eight inches distant. Near the position occupied by the stage of the microscope a side carriage is arranged which has a limited travel to and fro, and upon which are fittings to receive the metal bar when illumination of an opaque object is desired. The position of the bar carrying the optical apparatus can be adjusted from the end of the baseboard which supports the focussing-screen of the camera. Although this arrangement seems an unnecessary elaboration, it may under special circumstances prove of value. In any apparatus, however, the microscope and illuminant should be arranged in such a way that no further adjustment is necessary when they are swung into position in relation to the camera and are therefore ready for photographing. The fine-adjustment of the microscope is manipulated from the focussing-screen of the camera by means of a milled head substantially in the same manner as already described with the simpler apparatus. The chief characteristic of this apparatus is clearly the method of swinging out the optical part of it so as to make it available for work with opaque objects.

A more complete apparatus and one embodying arrangements 


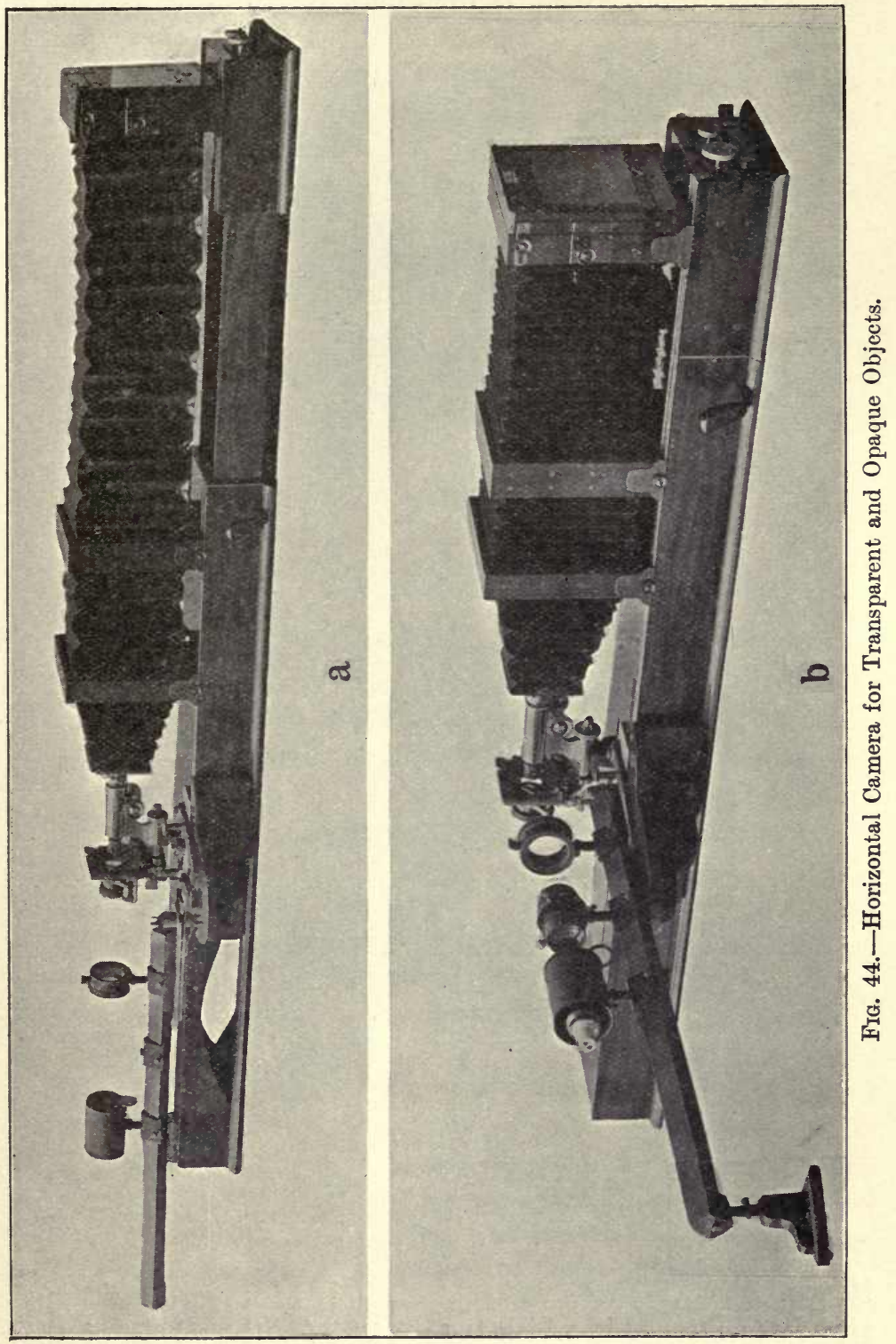


and founded on principles which differ somewhat from those already described, is the photo-micrographic camera of

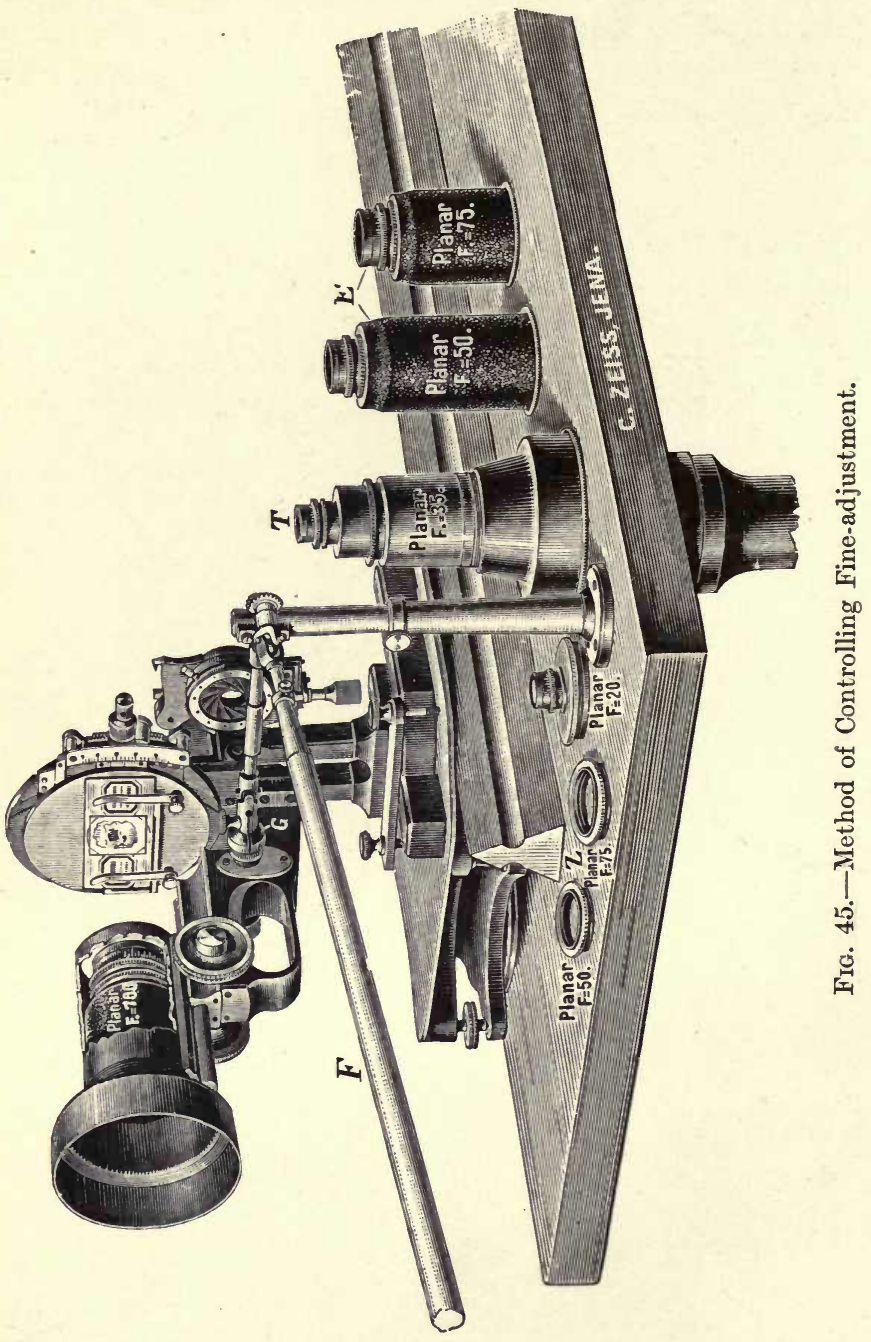

Messrs. Zeiss (Figs. 45 and 46). This is of the second type already mentioned in which the support for the whole of the optical arrangement is separated from the camera and its support; there are, therefore, two separate pieces of apparatus. 
The microscope is carried on an adjustable metal plate, which allows of its sliding at right angles to the optical axis, hence it can be moved out of position for any adjustment to be made without interfering with the remainder of the apparatus. It consists of two tables, both adjustable for height, one carrying the camera and the other a triangular bar upon which all the optical parts as well as the illuminant can be fixed, although in

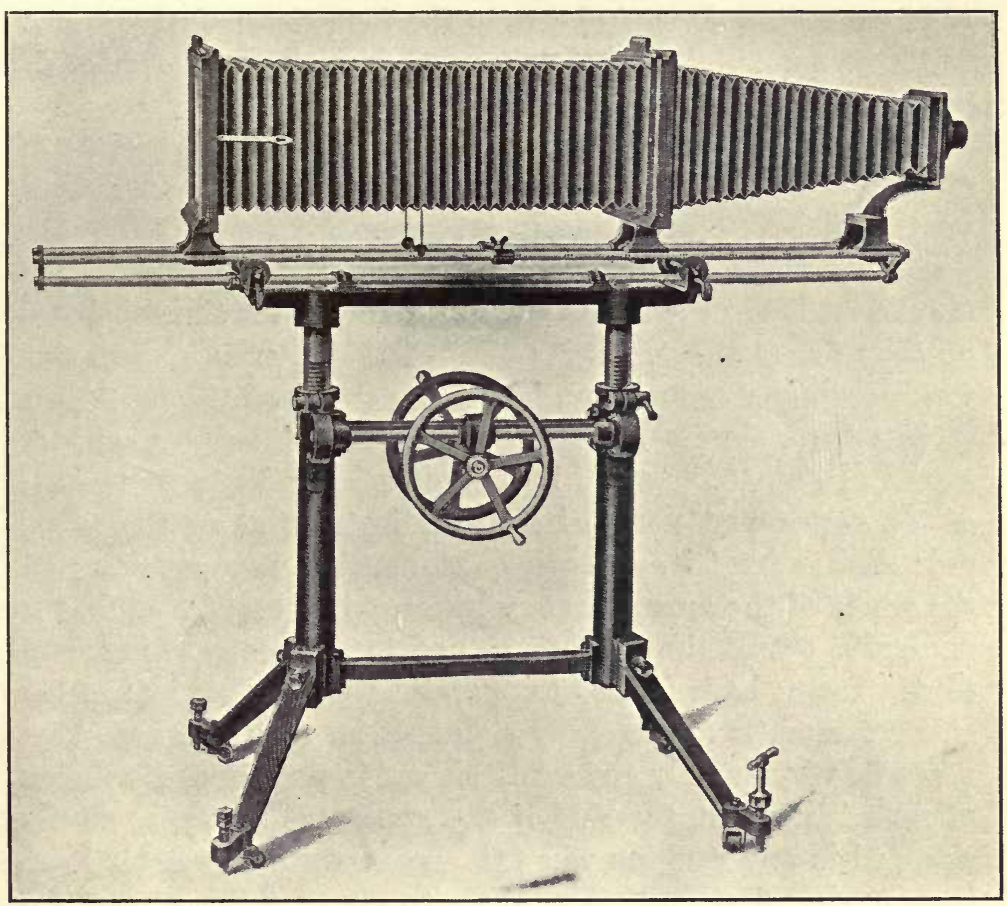

Fig. 46.-Large Micrographic Camera. (Zeiss.)

later forms the latter is supported on the table at the end of the triangular bar. All the optical apparatus is carried on saddles which may be adjusted and clamped in any position on the triangular bar. The sole-plate carrying the microscope also has levelling screws and elevating gear, so that any microscope of less height than that for which the apparatus is designed may also be used on it. The method of focussing from a distance-that is, from the position of the focussing- 
screen of the camera-is by means of rigid rods of wood or metal which are connected together by means of flexible joints (Fig. $45)$; there are no cords or similar flexible connections as in the apparatus previously described. A bell-shaped metal cap slips over the fine-adjustment screw and can be clamped on to it. An arm in connection with this clamp goes to a column, adjustable for height, which is fixed on the table at the side of the triangular bar; by means of a Hooke's joint a flexible yet rigid connection is made between the focussing-rod which goes to the camera, and the rod connected with the fine-adjustment of the microscope.

The illuminant supplied with the apparatus consists of an automatic electric-arc lamp. This is enclosed in a wellventilated box, and there are arrangements for adjusting the position of the crater to the optical axis of the apparatus. The illuminant is not carried on the metal bar, but is simply placed on the table at the end of it; the collecting-lenses, as well as a water-cooling chamber, are, however, carried on the bar. The camera-base, on its support, is constructed substantially as shown in Fig. 46. As will be seen, it is so designed that either of the component parts of it are adjustable along the metal rods that support it, and can be clamped in any position; or the whole of the apparatus can be moved to and fro so that it is easy to get at the microscope and to make any adjustment to it. In the later forms of apparatus the displacement of the entire camera is arranged by sliding it on two rollers, so that it can be very quickly and easily altered in position. By means of milled heads at the side of the apparatus it can be firmly clamped in any position when the necessary adjustments have been made. It is almost needless to say that this apparatus in common with all such produced by this firm, is, in point of construction, of the highest class. It must be admitted, however, that from its design it is rather susceptible to the influence of vibration. The camera is stiffly supported so far as its base is concerned; but as the lateral supports are metal rods, which are not cross-braced in any way, they are specially liable to feel the effect of any tremor imparted to them. At the same time, some of the finest work yet produced has been done with this apparatus; but it is essential to see that the position in which it is set up is as free as possible from any 
influence likely to cause relative movement of any of the parts.

An arrangement of practically the same design as the above is made by the Bausch and Lomb Optical Company of Rochester, U.S.A. It embodies all the main principles of the Zeiss apparatus, but is supported somewhat more strongly, and admits of the microscope and the optical accessories being lowered sufficiently for the instrument to be used in a vertical position. In essential respects this apparatus differs but little from the one by Zeiss, already described.

Nearly all firms supplying microscopes now make an apparatus for photo-micrography which embodies most of the points already enumerated. The large apparatus made by Messrs. Swift is after a model designed by Mr. Andrew Pringle, and therefore, it is needless to say, founded on the result of very wide and long experience. The whole apparatus is carried on one base, and the only point which is not mentioned in some previous type of instrument, is that the front portion of the microscope, when it is thrown into the horizontal position, is supported on a triangular trunnion. Therefore, the weight of the body-tube of the instrument is not taken entirely by its own tripod base; nor is the tendency to vibration or flexure of the apparatus so great when the microscope is of a very heavy and substantial design.

In most modern instruments this addition is not necessary; in fact, it should not be necessary, as the design should be such that the instrument is well balanced in either the horizontal or vertical position. Further, as the tendency of late is to simplify and reduce microscopes in weight and size, any additional means of support for the body-tube is not advisable. In other respects this apparatus is substantially made, and is capable of doing work of the highest order.

Similar types are also made by Messrs. W. Watson \& Sons, Charles Baker, and R. \& J. Beck; but as none of these embody points that are entirely novel or peculiar to themselves it would not appear to be necessary to set out a detailed description of them here, but reference should in each case be made to the makers' catalogues.

The type of apparatus used by the writer differs in many particulars from any of those hitherto described, and as it 
embodies certain ideas founded on mechanical principles that are not very generally appreciated, some description of the arrangement may be of interest.

The apparatus was originally designed by Dr. T. A. B. Carver and the writer for use at the Lister Institute of Preventive Medicine, and the objects aimed at were to combine perfect rigidity with ease of manipulation. The supporting portion of the apparatus consists of two light iron girders which are very strongly braced together (Fig. 47). On the top of these, two steel rods are supported, and the camera slides to and fro along them. Any part of the camera is adjustable along the rods, and can be very firmly clamped in any position. The bellows are made in segments, so that a very short camera or one of a length up to six feet may be used as required. The microscope, illuminant, and other optical parts are supported on a tail-piece which may be swung out on a central pivot, allowing of convenient access to the microscope (Fig. $47 \mathrm{~b}$ ). The end of this tail-piece nearest to the camera is supported on a semicircular arm, which projects out from the side of the cast-iron frame, and which serves as a support for the front end of the tail-piece when it is swung out of alignment with the camera. The illuminant, collecting-lenses, and the microscope are carried on a triangular metal bar, as in the Zeiss apparatus already described, and each unit of the apparatus is on a saddle-piece, which allows of adjustment in a vertical direction and in the line of collimation, and may be clamped in any position.

There are two methods of focussing which may be adopted alternatively. In one a long steel rod runs along the entire length of the base of the instrument between the girders, and is provided with movable brass milled heads at intervals, so that focussing can be effected from any position of the focussingscreen of the camera. At the end of this rod, immediately under the pivot which supports the swinging-out tail-piece carrying the microscope and illuminant, is a grooved pulley. Over this pulley a waxed silk cord is carried, and uniform tension of the cord is maintained by means of a weight at each end. This cord passes through a central hole in the pivot supporting the swinging-out tail-piece, and is led up to a double-grooved pulley which is on the table supporting the microscope, and 


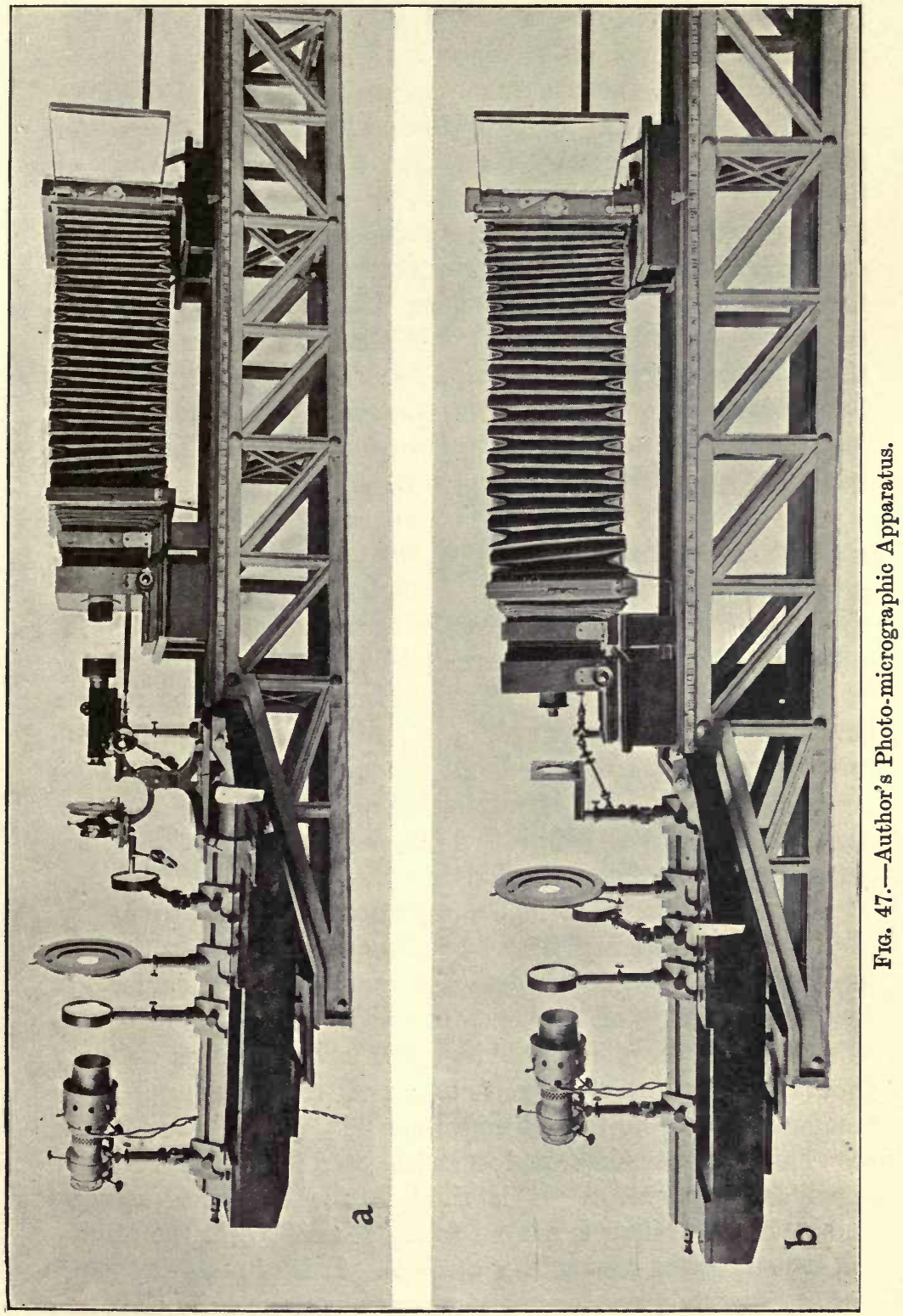


is immediately underneath the position of the fine-adjustment. Between the fine-adjustment and this double-grooved pulley an endless silk cord passes which has very slight tension on it, and is only sufficiently tight to ensure movement of the fineadjustment when any motion is imparted to the pulley. As already explained, a very considerable degree of slackness of the silk cord is permissible and, if it is well waxed, the tension on the fine-adjustment is reduced to a minimum. The arrangement further imparts a very smooth and regular motion to the fine-adjustment on rotating either of the milled heads on the long steel rod, and it has the additional advantage that, owing to the connecting-cord passing up through the hollow centre on which the tail-piece revolves, no part of the focussing apparatus requires to be detached if the microscope has for any purpose to be swung out.

The second and alternative arrangement for focussing is the method already described as attached to the Zeiss photo-micrographic apparatus (Fig. 48). In actual working it does not appear to have any advantage over the method above described, although perhaps the response of the fine-adjustment to any rotation of the focussing-rod is more immediate in the case of the Zeiss method, whereas with any arrangement in which there are cords there is always some slight stretch of the cord itself on reversing the direction of rotation which has to be allowed for, and which happens before the fine-adjustment motion comes into play. The Zeiss method has the distinct disadvantage, that if any adjustment of the microscope becomes necessary, and the tail-piece is revolved to allow this to be effected, the focussing-gear has to be detached, and this is of course not necessary with the method first described. The whole apparatus may be used for photographing with low powers or ordinary photographic lenses. Fig. $47 b$ shows it so arranged, the microscope and its table being removed.

This apparatus in actual work has been found to fulfil every possible requirement. It is rigid in the extreme, and any vibration or tremor conveyed to it does not affect the results, as the apparatus responds as a whole. It is of necessity some. what expensive, and for many purposes unnecessarily elaborate to build up in the way described and illustrated. It is, however, important to recognise that it is designed entirely on the 
geometric slide principle, and does not depend for its accuracy on very fine workmanship. On this principle a very simple and inexpensive apparatus might easily be arranged, and would answer almost as well. The first model apparatus of this type was built up entirely of wood, the girders being made in the form shown, and the results obtained were entirely satisfactory.

The writer cannot too strongly recommend anyone who may contemplate building up an apparatus to his own ideas

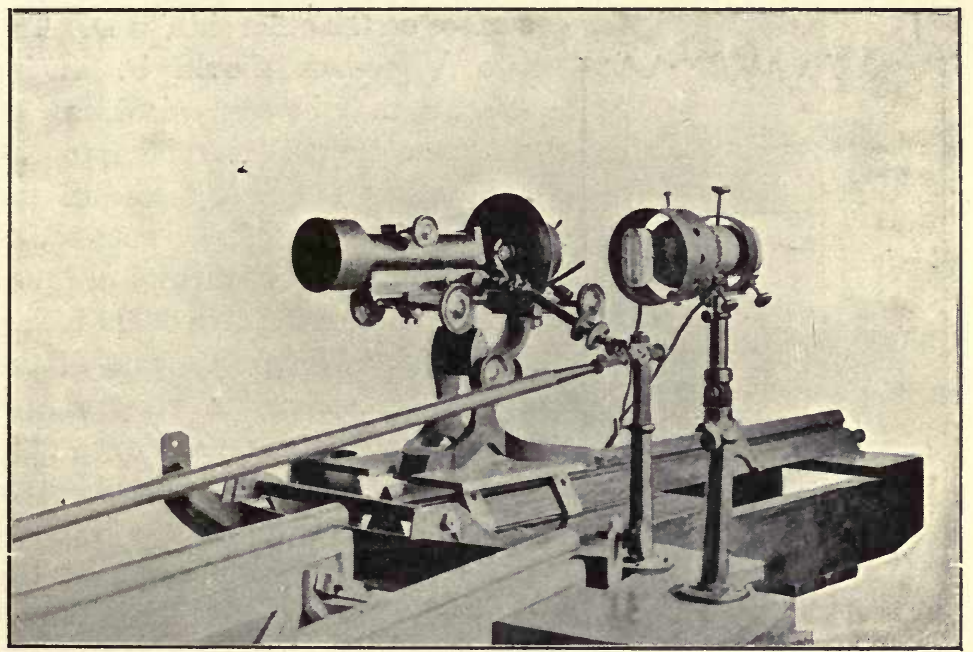

Fia. 48.-Author's Photo-micrographic Apparatus.

to study carefully. the principles of the geometric slide as enunciated by Lord Kelvin. ${ }^{1}$ It is only recently that this method has been appreciated in the construction of scientific instruments, and although it is admitted that for heavy machines it might have disadvantages, for any purpose such as is now being discussed, it far surpasses any other method of design or construction. The accuracy in working of any apparatus so built is far less dependent on perfect workmanship than in any other design.

Fig. 49 illustrates the geometric slide and the method by which it may be made. Let B represent the baseboard of the

1 Thomson and Tate, Treatise on Natural Philosophy, part i, p. 154. 
proposed camera, on which are fixed two metal rods or tubes A, A (these should preferably be brass-cased iron tubes of about three-quarters of an inch in diameter, as they are stiffer and cheaper to purchase than rod or tube of solid brass). Any part of the apparatus that

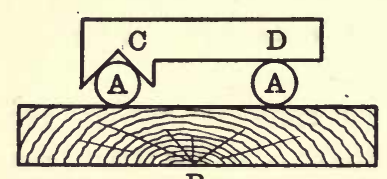

B

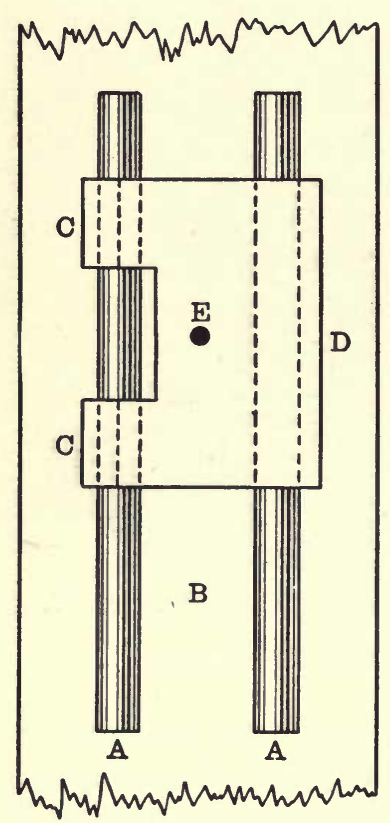

Fig. 49.-The Geometric Slide. requires to slide along these rods should be supported on a piece of wood which has two V-shaped grooves (as C, Fig. 49) in line along one side of it, and a plane surface on the other side, as D. The two $\mathrm{V}$-grooves should be as far apart as may be convenient, so that a longer support is given to the apparatus; but in the case of the focussing-screen of the camera, or any similar part, then a distance from end to end of five or six inches is ample. When any part of the apparatus sliding in this manner requires to be clamped down, only one screw is necessary, and this should pass through the slidingplate as nearly as possible at the centre $\mathrm{E}$ of a triangle which is formed by the ends of the two $\mathbf{V}$-groove slides and the centre of the plane point of support on the other side. No part of this arrangement requires to be made with any particular accuracy. The two faces of the V-grooves are preferably at right angles to one another, so that when clamped down there is no tendency to jam, and also to ensure that the movement is sufficiently free when the clamping-screw is released.

In placing any piece of apparatus on such a geometric slide advantage should be taken of the method-also set out by Lord Kelvin -of three points of support. If any piece of apparatus resting on three points is set on a horizontal plane it then only 
has freedom to rotate on a vertical axis, and when clamped down has no power of motion perpendicularly to that plane. The ends of the three points of support should be rounded, and one of these should be dropped into a shallow conical hole; the apparatus then only has freedom of rotation about this one point. The second point should then be placed in a $\mathbf{V}$-groove set in a position so that the groove runs in a direction away from the centre of the triangle formed by the three feet of the apparatus. The third point of support is on a plane surface. It is clear that any apparatus standing in this manner has no freedom to move, and if it is taken off its base for any purpose, will, on being placed again in its required position, be absolutely in the same place it was in originally. It is very difficult indeed to claim this for many of the methods of supporting apparatus which are now in general use, and although the objections to their designs may to a large extent be overcome by good workmanship, they would never reach the same degree of accuracy that can be obtained by following out this method of the geometric slide and clamp. The various parts of the apparatus made by the writer are shown in Figs. 47 and 48 ; and, although the apparatus itself looks somewhat complex, nearly the whole of it has been constructed in an amateur's workshop, and by one who is by no means an expert mechanic. In a simple form of apparatus all that is really necessary is to have a baseboard to support the whole, and two long stiff metal rods on which all the components of the apparatus can slide.

The camera may be supported at either end on the Vgrooves and plain slide, and clamped down as described. For each piece of subsidiary apparatus, and for the microscope, a wood table can be made in the same way to slide on these rods. In the case of the microscope it should be placed on three points as described, so that it always returns exactly to its proper position. Any other apparatus, such as the illuminant or subsidiary collecting-lenses, may be supported on small sliding tables in the same manner, so that every part of the apparatus is adjustable, and can be clamped down in any position. Such an apparatus could be constructed very simply at small cost.

Fig. $47 b$ shows the adaptability of the apparatus for photographing large objects, either with ordinary photographic lenses or with low-power microscope objectives. The object-carrier 
is shown in position near to the objective on the camera-front, the illuminant and bull's-eye condenser occupying a position

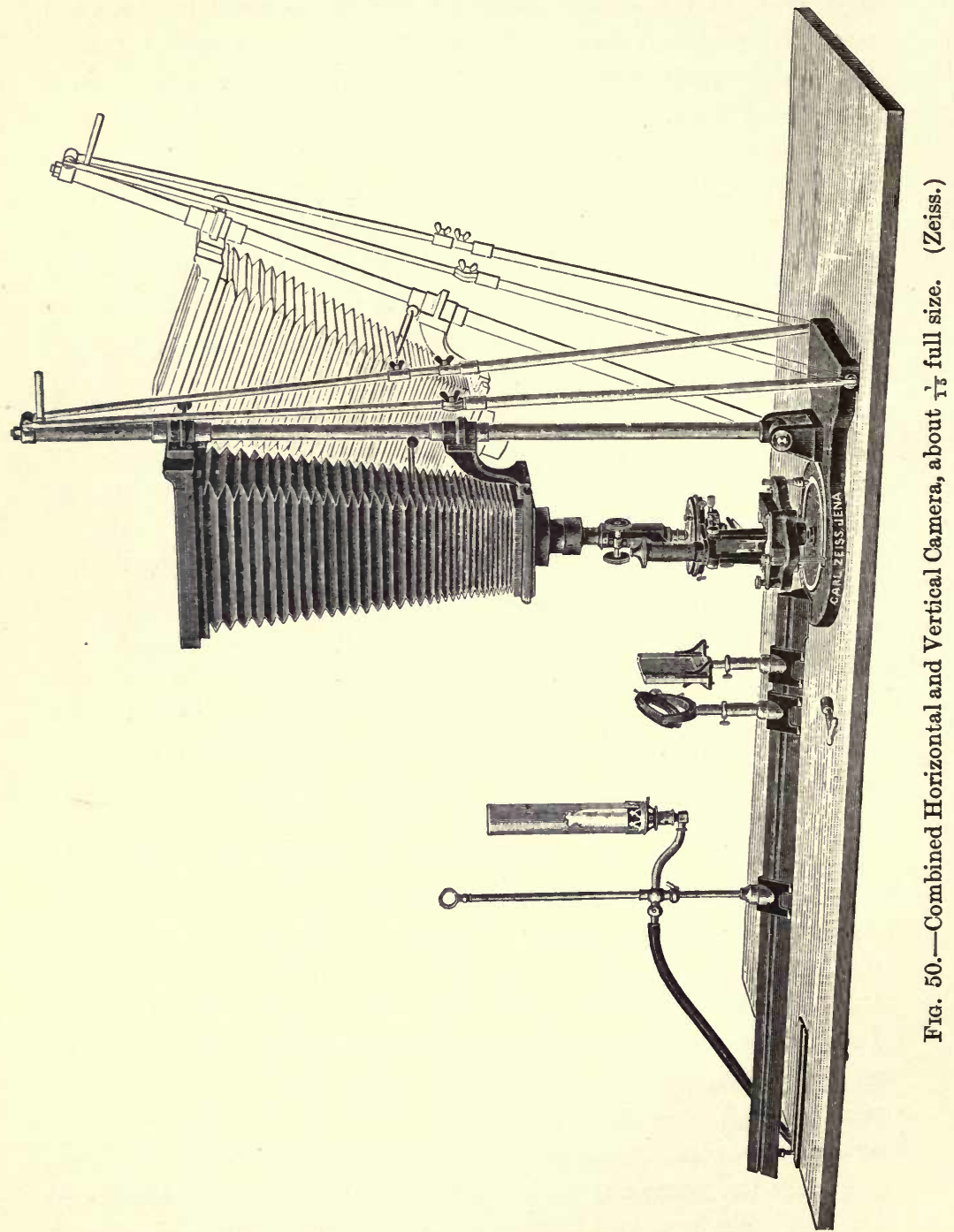

on the triangular metal bar as shown. With the geometricslide principle carried out entirely, this triangular bar is of course not necessary, the whole of the moving parts being carried on the two metal rods as described. 
Combined Vertical and Horizontal Cameras.-Various types of camera have been devised embodying the two designs vertical and horizontal, and arranged so that the camera may be used in conjunction with the microscope in either position. None of them can be said to be entirely satisfactory, except one which has been recently brought out by Messrs. Zeiss. In this new apparatus an efficient arrangement is made for overcoming the great objection to all vertical cameras which are supported on a single metal bar. This is effected by attaching struts to the supportingrod so that it has no freedom to vibrate. The accompanying illustrations (Figs. 50 and 51) show this.

The microscope and camera are supported on one single solid base, and the arrangement is such that with ordinary care the act of removing the shutter in the darkslide of the camera should not result in any displacement of the various components.

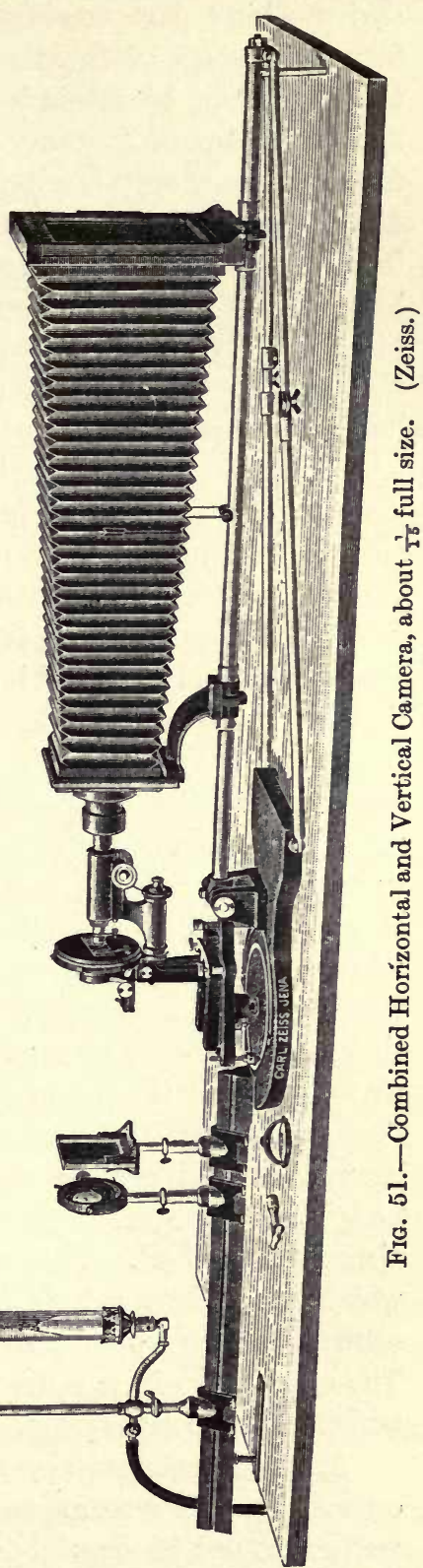
The base consists of a heavy cast-iron plate separated into two 
parts, the front circular portion supporting the microscope, and the hind part serving as a support for the camera. The focussing-screen of the camera as well as the front are adjustable, and can be set in any position on the supporting-rod, and by means of a groove in this rod the parts are prevented from rotating, only having freedom of movement in a vertical direction. The bellows are fixed to the frame carrying the focussing-screen by a hinge and catch, so that if the catch is loosened, the bellows drop down at one side, and the image of the object may be viewed on a dead-white reflecting-screen if desired. Vibration of the supporting-rod is prevented by having two telescopic struts which are supported at their lower end from the corners of the base-plate, and which can be loosened when any alteration of camera-length is necessary. These may then be clamped at any position when the desired length of camera is obtained.

When arranged similarly to Fig. $47 \mathrm{~b}$, but in the vertical position, it is also available for photographing large objects, any suitable lens simply being screwed on to the front of the camera in the ordinary way. An additional camera-front is supplied for carrying the flanges of any such lenses. Two focussingscreens are supplied, one ground-glass and the other plain glass, as with other cameras. The base-plate supporting the microscope rests on three levelling-screws, and can be clamped down when once the microscope has been adjusted in position in relation to the camera. It can also be entirely removed should the camera be required for other purposes. This baseplate, carrying the microscope, can also be rotated so that the camera is available on either side of the operator. The illuminant and optical accessories are carried on the optical bench in the same way as in the larger horizontal camera already described. The optical bench has a pin at one end which drops into a hole in the base-plate, so that it always returns to its proper position in relation to the microscope. The other end of the optical bench is supported on the table on which the apparatus rests.

Arrangements are also made for altering the position of the optical bench according to whether the camera is required in a vertical or in a horizontal position. It may be used in the horizontal position for macrophotography, for the photographing of 
bacteriological culture-tubes, or for large objects which are not so easily dealt with when the camera is in the vertical position.

The procedure when changing the camera from the vertical to the horizontal is quite simple, and is effected by loosening the clamps on the vertical rod, and also on the bracing-stays, which at once allows the camera to be tilted over. The accessory apparatus on the optical bench then has to be brought to the proper height so that it is in alignment with the optical centre of the microscope; the adjustments are the same as in other types of horizontal apparatus.

The extension of camera in this apparatus is not sufficiently great to render it necessary to provide any additional focussing-rod for operating the fine-adjustment, so that to obtain high magnifications it will generally be necessary to use higher power projection-oculars.

\section{Cameras for Special} Purposes. - With the recent introduction of improved arrangements for illuminating living

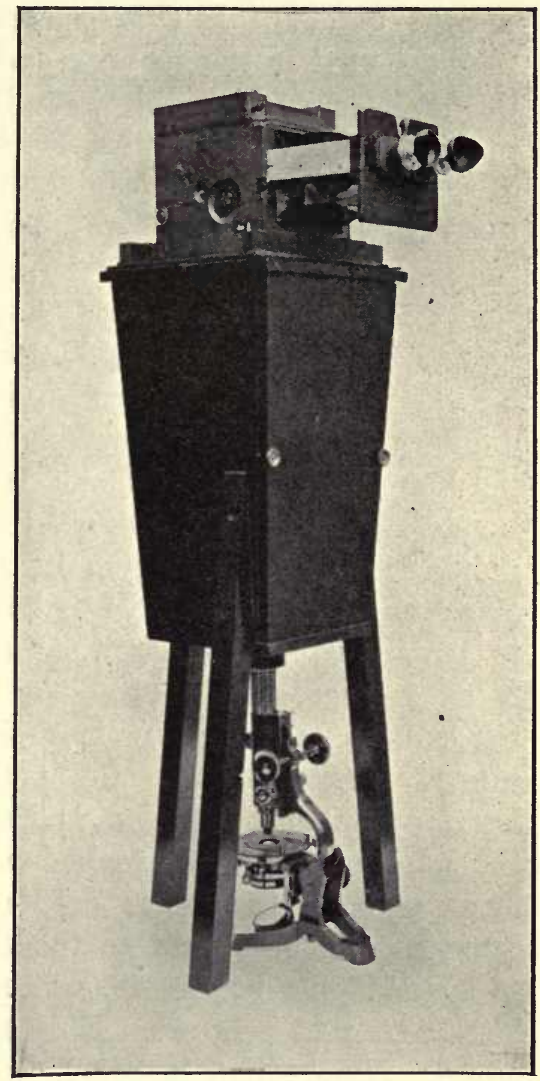

FIG. 52.-Reflex Camera used in combination with Van Heurck Vertical. bacteria and other unstained objects, by means of the parabolic illuminator of Messrs. Zeiss and the one made by Messrs. Leitz to fulfil the same purpose, it has become desirable at times to have an apparatus which shall be capable of use for photographing such living and moving objects. The essential point 
about such an arrangement is that it should be possible to observe the microscopic image, and to focus up that image until the desired appearance is seen; then to be able to take an instantaneous photograph at the exact moment when this appearance is observed and the object is in focus. A simple way of doing this is to utilise an ordinary reflex photographic camera (Fig. 52). In these cameras, it is well known that when they are used in the manner and for the purpose for which they are designed, an object can be seen and focussed right up to the moment of exposure. For photo-micrographic work one of these may be used in conjunction with a vertical camera of the Van Heurck type already described. It is obvious that a camera of the vertical type is the one most useful for this class of work; for it almost invariably happens that living microscopic objects are immersed in fluid, and that therefore it is essential for the microscope to remain in a vertical position. The ordinary ground-glass focussing-screen at the upper end of the Van Heurck camera should be removed, and in its place a wooden frame to carry the reflex camera is fixed. This frame should have an opening in its centre-say, of three inches diameter-which is sufficiently large for any microscopic olject to be projected through it. The photographic lens on the reflex camera, and if possible the whole of the front of the camera carrying the lens, should be removed, and the camera turned over in the position as shown, so that it lies in a horizontal position, and allows the fine-adjustment of the microscope to be reached while observing through the focussing-hood the image on the ground-glass screen of the camera. The only limit to the employment of this method, so far as higher powers are concerned, is the intensity of the illuminant that is in use. It is obvious that with any darkground illumination method, to obtain an instantaneous photograph the light-source would require to be powerful, and it is very doubtful whether anything less than an electric arc would be of service. In any case the camera would require to be used in conjunction with one of the high-power illuminating methods already described. It is essential that the reflex camera utilised for this purpose, although it may be of very simple design, should be such that it does not cause any appreciable amount of vibration on releasing the reflecting 
mirror and effecting the exposure; also that the period elapsing between the release of the mirror and the actual exposure of the plate should be as short as possible, so that if any vibration is started, it will not have time to be transmitted to the rest of the apparatus.

An elaboration of the same idea and an arrangement suitable for use in conjunction with a large horizontal apparatus, such as that made by Zeiss already described, has recently appeared. 1 It consists essentially of a reflex camera of special design which is placed in a position between the microscope and the focussing-screen of the camera, occupying, in fact, the place usually taken by the conical front of the camera. The image is viewed in a position above that where connection is made between the microscope and the camera, so that double reflection of the image takes place between two mirrors placed in a suitable position inside the reflex portion. It was designed for the same object as the vertical apparatus just described, but it appears to be open to the objection that it is not available for the photography of objects in fluid, and in which therefore the microscope must remain in a vertical position. In other respects it would no doubt fulfil its purpose perfectly. Its chief advantage appears to be that the operator is able with ease to adjust both the coarse- and fine-adjustment focussing-screws of the microscope; and also at will to effect any movement of the stage or alteration of the sub-stage illuminating apparatus, while at the same time being able to see the result of any alteration of these adjustments on the ground-glass screen.

For metallurgical work, or the systematic photographing of opaque objects, an arrangement must be made for supporting the source of light at right angles to the optical axis, so that a vertical illuminator or any other arrangement for opaque illumination may be used. This can be simply arranged by having a support on either or both sides of the base carrying the camera, or on any table or bench that is carrying the apparatus. - It is convenient, however, in any case to arrange that the illuminant is so supported that it is possible to alter the distance between it and the illuminating apparatus while keeping it in perfect alignment.

When using the auxiliary illuminating appliances that are

1 British Journal of Photography, March 12, 1909, p. 193. 
carried on an optical bench, it must be arranged that the latter can be either swung out at right angles, or that an additional carrying-bar can be placed in the desired position.

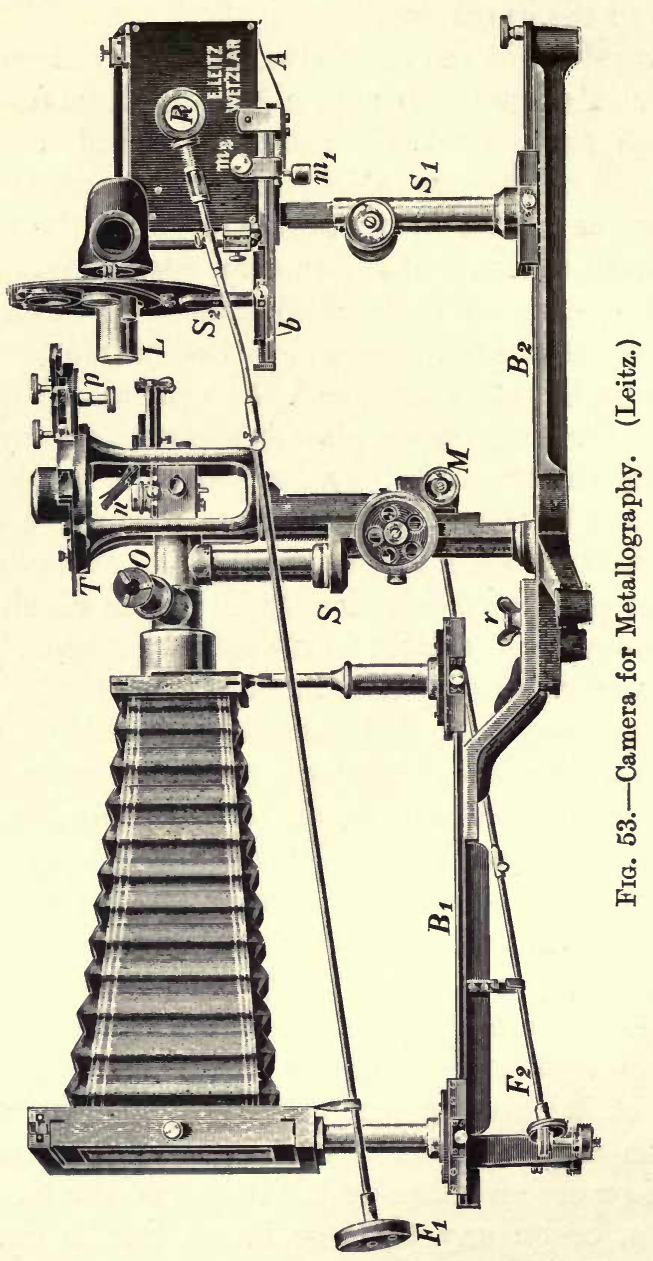

An entirely new type of apparatus for metallurgical work is one recently introduced by Messrs. Leitz, and known as their micro-metallograph (Fig. 53). This apparatus is designed for visual observation, as well as for photo-micrography. Its novelty consists in the manner in which the object is supported in 
relation to the optical system. The stage is situated in the position $\mathrm{T}$, and any object that is to be examined is placed on it with the surface under observation downwards. Focussing is effected by moving the stage, the objective and the rest of the illuminating system remaining stationary, and this constitutes its chief feature; the manner in which this is effected is a distinct departure from existing practice. The apparatus consists essentially of three parts, all of which are mounted on an optical bench, viz. a stand with a tube carrying the objective and illuminating system, and the object-stage ; an electric lamp, usually a small are light with an iris-diaphragm and illuminating lenses; and a photographic camera. The upright $\mathrm{S}$ carries a tube for observation together with the illuminating appliance, and also has on it all arrangements for altering the various adjustments. The stage $\mathrm{T}$ is raised and lowered by means of a rack and pinion, and also has a fineadjustment fitted to it as with an ordinary microscope. The lamp is carried on a supporting upright $\mathrm{S}_{1}$, and is adjustable in a vertical direction by means of a rack and pinion, and adjustment is provided for centration of the are itself, if that be used, by means of the adjustment-screws $m_{1}$ and $m_{2}$. The arrangement is really intended for use in conjunction with the small Leitz are lamp already described, in which the carbons are at right angles to one another. The camera portion itself has no special feature: it is made in three sizes, capable of extension to 28,50 or 100 centimetres, and entails the use of a fairly high-power eye-piece when high magnifications are required. The camera is supported on two uprights which are adjustable in the line of collimation. A mechanical stage is fitted, and two milled heads impart to the stage a compound cross motion, and the amount of this motion may be read to one-tenth of a millimetre by means of two vernier scales. As a means of finding any given spot on a piece of metal the mechanical stage is provided with a sliding pointer, the position of which can be ascertained and registered by a scale. The mechanical stage in the later forms is built into the apparatus to ensure greater rigidity. The opening in the stage may be varied in size so that large or small specimens can be supported on it.

The following directions are those given by Messrs. Leitz for 
guidance in using this apparatus and in making the necessary adjustments ; they sufficiently clearly indicate its purpose, and the objects to which it may be applied (Fig. 54).

Let B mark the position of the source of light, and $\mathrm{J}$ represent the iris-diaphragm. Adjust the position of the lens $\mathrm{L}^{\mathrm{l}}$ within the tube until an image of the iris-diaphragm is formed at E, i.e. about midway above the upper face of prism P. The latter may be turned about an axis at right angles to the plane of the paper and also displaced along $\mathrm{AB}$.

The pencil of rays proceeding from $\mathrm{P}$, after passing through the objective $\mathrm{O}$, illuminates the object, whence that portion

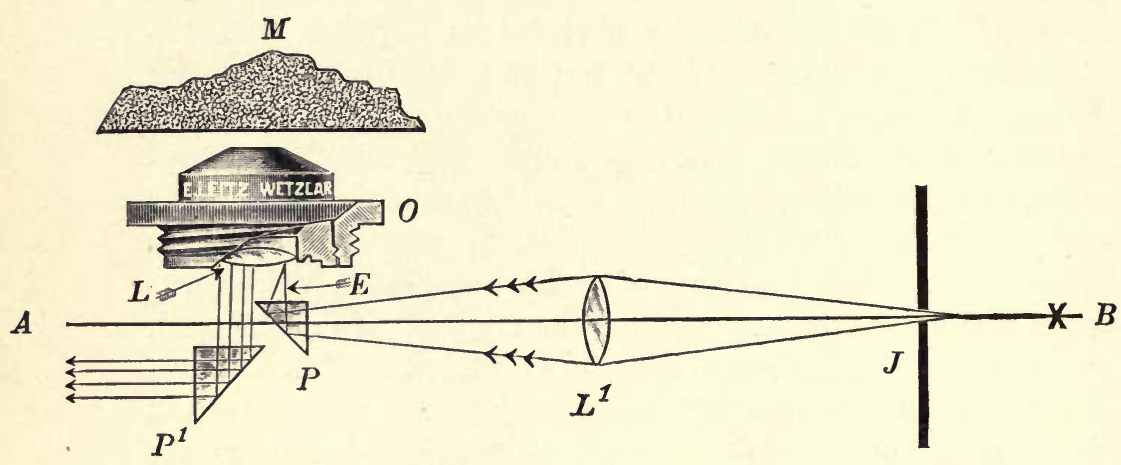

FIG. 54.-Diagram of Path of Rays in Vertical Illuminator. (Leitz.)

which goes to form the final image in the eye-piece or on the ground-glass plate is reflected back through the objective, past the prism $\mathrm{P}$, and through prism $\mathrm{P}$.

The movable mount of prism $\mathrm{P}$ affords a means of so directing the rays through the objective $O$ that any light which is reflected by the lens $L$ may be intercepted by prism $P$. The light does not then pass through the prism $\mathrm{Pl}^{1}$, and no disturbing glare is created. The width of the pencil emerging from the prism $\mathrm{P}^{1}$, which is of considerable importance, may be regulated by displacing lens $\mathrm{Ll}^{\mathrm{l}}$, which to this end is contained in a movable mount, the distance of $\mathrm{L}^{1}$ from $\mathrm{P}$ being greater with high than with low powers. To direct the correctly illuminated portion of the object into the field of view it is only necessary to turn $\mathrm{Pl}^{1}$ about its axis.

Having completed the adjustment of the illumination, the 
operator may make sure of the absence of glare, which is particularly disturbing in photographic work, by removing the object and replacing it by a non-reflecting body, preferably black velvet. The field, as seen through the eye-piece, should then be black.

After a little practice this test will become superfluous, since slight glare will be readily recognised in the course of the manipulations described.

Having secured the proper adjustment, as controlled through the eye-piece tube, put the latter out of action and let the image appear on the ground-glass focussing-screen. Photographs are taken by this apparatus with the aid of an eye-piece as well as the objective, a fitting containing an eye-piece being inserted into the sleeve attached to the camera. The final focussing and the adjustment of the carbons is effected with the aid of the transmission gears $\mathrm{F}^{1}$ and $\mathrm{F}^{2}$ (Fig. 53).

The image, as seen in the observing eye-piece or on the ground-glass screen, should be evenly illuminated, bright, and undistorted. The presence of distortions should be regarded as an indication that prisms $\mathrm{P}$ and $\mathrm{P}^{\mathrm{l}}$ are not properly adjusted. 


\section{CHAPTER VII}

\section{THE USE AND MANIPULATION OF THE MICROSCOPE}

Essential Adjustments. - It is, above all things, necessary that the microscope and its optical parts should be in perfect adjustment, as on this more than on any elaboration of apparatus will depend the excellence of the results. It does not come within the scope of this work to go fully into the theory and practice of microscopy, and reference must be made to one of the standard works-a list of which is given in Appendix VI -if fuller information is required. Sufficient will be said, however, to enable one who already has some knowledge of the microscope and its use, to make the necessary adjustments. The matter will be treated from the standpoint that the optical portion of the microscope consists essentially of the three parts already enumerated :-

The objective, which forms the actual microscope image;

The eye-piece, which more or less amplifies this image;

The sub-stage condenser, to illuminate the object efficiently.

It will be assumed that some very simple object presenting no particular difficulty, preferably without colour and the image therefore only being formed as the result of light obstruction, is first placed upon the microscope stage. A low-power objective-for example, a 1-inch - should be screwed into position on the instrument. The sub-stage condenser had better for the moment be removed and illumination be effected by reflecting light from the plane side of the mirror. The illuminant may be placed in a convenient position-say, some eight to ten inches away from the mirror - and the latter adjusted so that the light is reflected on to the object. On observing the image 
through the eye-piece of the microscope an evenly illuminated field results.

In any case, before taking a photograph this operation with greater or less elaboration will be necessary, so that the part of the object required in the field of view may be brought into position.

In observing the image, if the worker has not already accustomed himself to do so, he should at once endeavour to train himself so that both eyes always remain open. This applies not only when observing the image visually, but also when focussing, the image being projected on the ground-glass screen of the camera and observed with a focussing ocular. At first this may seem a trifle difficult, as extraneous objects become painfully evident. But it will be found with some little practice, especially if the eye not in use is for a time partially shaded with the hand or with a piece of black cardboard, that it soon becomes an easy matter to leave both eyes open. Both eyes should be used alternately, so that there is no greater strain on one than the other. Where long searches have to be conducted it is a great relief to change from one eye to the other at short intervals, thus avoiding the risk of any undue strain and consequent fatigue.

The object having been set up, the most instructive procedure is to turn the microscope into the horizontal position and, instead of viewing the image directly, to project it on to a small white screen, such as a piece of white cardboard some eight or ten inches square, placed, say, at a distance of eighteen or twenty inches from the ocular. The mirror must of course be either swung aside or removed. It is with projected images that in photo-micrography the worker is almost entirely concerned, and some preliminary experiments in simple projection will be far more useful than to begin at once exposing plates.

When the microscope is in the horizontal position, assuming that the object has been approximately focussed and the source of light placed so that it is properly illuminated, the projected image will be seen on the white screen placed as suggested. It may be found that in any case some alteration in the position of the illuminant will be necessary before the field becomes evenly illuminated. It will somewhat facilitate perfect centration if the iris-diaphragm be now closed, so that 
only a small point of light is allowed to pass. Should there be any want of centration in the position of the light, this will now become evident, and a much smaller error is at once seen if the iris is closed than when it is wide open. Having effected the centration the iris must then again be opened, but only suffciently to well illuminate the object. The bull's-eye condenser, or a similar auxiliary condensing-lens, may then be interposed between the source of light and the object. It is important to see that this lens is placed so that it is exactly at right angles both in a vertical and horizontal direction with the optical axis. It will be found that it is in its best position when either an approximately parallel or a convergent beam is projected on to the stage of the microscope, or on the iris-diaphragm underneath the stage. Some movement to and fro of the bull's-eye condenser will no doubt be necessary to obtain this result. The condition, therefore, is that the illuminant is approximately at the principal focus of the bull's-eye condenser, which should present its plane side towards the light, and the object is evenly illuminated with a parallel beam. The iris-diaphragm on the microscope should then be contracted until no more light than is necessary reaches the object, and so that the size of the illuminated area is no larger than is required. It should be noted that to obtain a parallel beam the bull's-eye must be near to the source of light, and must be moved away from the light to obtain a convergent one. It may be useful to alter the position of the light or of the bull's-eye in relation to the microscope and watch the unevenness of illumination that results from any want of centration. It is on this point as much as on any other-the correct centration of the light and auxiliary condensing-lens-that good results depend in any low-power work.

It is assumed that the worker is conversant with the appearances of an ordinary projected photographic image, such as may be seen in any ordinary camera and is projected with an ordinary photographic lens. If he is not, however, then the preliminary work had better consist of the observation of such an image without a microscope at all, but using an ordinary photographic lens on the front of his photo-micrographic camera. He may then with any relatively large microscopic object perform the same operation of illuminating the object and 
getting an evenly illuminated picture as already described with a low-power objective on the microscope.

This experimental method of observation of the projected microscopic image may then be extended farther, and a rather higher power objective put on to the microscope-say, a halfinch-the sub-stage condenser replaced, and the illumination watched as before. The upper lens of the sub-stage condenser should be removed, if its construction will admit of that, so that the image of the illuminant is larger and covers a larger field.

The illumination in this case may be best effected by removing the bull's-eye for the moment, then racking up the sub-stage condenser until an image of the illuminant is seen projected over the image of the object. The illuminant should in this case be about ten inches from the microscope sub-stage. This image of the illuminant should be seen quite in the centre of the field. Then again interpose the bull's-eye condenser, and arrange it as before, and in such a position that the field again becomes evenly illuminated. The effect of altering the position of the bull's-eye between the light and the microscope should be noted; but the change should be effected by moving the bull's-eye from the position in which it gives a parallel beam towards the microscope, so that it gives a converging one. To obtain an evenly illuminated field it is necessary in fact for an image of the aperture of the bull's-eyeitself to be projected into the object-plane. In cases where the bull'seye has an iris-diaphragm or a single metal plate with a small hole in it-an accessory often supplied with these appliancesit is easy to focus the image of this small aperture in the same plane as the image of the object by racking up or down the sub-stage condenser. On removing the stop the object will then appear evenly illuminated. If a parallel beam from the bull's-eye does not give enough light, a convergent beam may be used; but it should be so arranged that a roughly focussed image of the radiant is seen on the iris-diaphragm at the back of the sub-stage condenser. The illumination will then be sufficiently critical for use with an objective not higher than half an inch.

Further reference to this and instructions in detail will be given later when referring to the photography of typical objects; but this preliminary observational work will be found most 
useful in enabling one not conversant with the appearances obtainable to appreciate what may be done by alteration of position of the various parts in use.

The Use of Objectives and Oculars. - In ordinary observational work the use of the low-power objective presents no particular difficulty ; it is only necessary to screw the objective into its proper position on the microscope body-tube, use it in conjunction with a suitable eye-piece, and focus the objective on the object, when an image will appear. As a practical point, it is always advisable with low or medium powers to rack down the objective with the coarse-adjustment of the microscope, so that the front of the objective is in close proximity to the object. To bring the object into focus the motion of the bodytube is therefore away from the object. This is only to avoid the possibility of any injury to the specimen or the objective by racking down too far by means of the coarse-adjustment. With high-power objectives, and particularly oil-immersions, this is hardly practicable, as the working distance is already so small that to bring the objective still lower down is a matter of considerable difficulty. The safest way therefore is, having immersed the objective in oil, to carefully focus down until some particle of dust on the top of the cover-glass is in the field of view, and then by means of the fine-adjustment to work down until the object is seen. Incidentally it may be remarked that it is more satisfactory to place the cedar-wood oil for immersion purposes on the front of the objective itself, and then to lower this down on to the object, than to place the oil on the objectslide, as is so generally done. This is perhaps a small point, but it has a definite bearing on the work in hand, as it ensures the oil being in the proper position on the object, assuming that some preliminary centration has been effected with a lower power objective; and, again, it prevents the possibility of too large a quantity of oil being used. When the microscope is thrown into the horizontal position if there is any considerable excess of oil it tends to flow away, with the result that if it is very fluid the oil may leave theobjective and specimen entirely; and, as this may occur during an exposure, it would mean that the photographic result would be entirely spoilt.

Before inserting an ocular to use in conjunction with the objective it is important to see that the tube-Jength is that for 
which the objective has been designed. With low powers some small variation from the correct tube-length is permissible, but this does not apply to a medium or high power. It is unfortunate that makers have not adopted a uniform standard tube-length. Some compute their objectives to work with a length of 160 or 170 millimetres, usually referred to as the short-tube ; while others use what is known as the English tube - that is, a length of 220 millimetres. As explained later, some variation of these tube-lengths may, under certain conditions, be necessary ; but for low- or medium-power objectives, or for oilimmersions, it will be advisable not to vary the tube-length from that stated by the maker to be the correct one. The majority of objectives are now corrected either for 160 - or 170millimetre tubes; and all microscopes have sufficient length of tube to accommodate themselves to the required condition. It is important to remember that in adjusting this tube-length due allowance must be made for any objective-changer that may be on the microscope. This again varies considerably according to the custom of the maker. The Zeiss sliding changers add 22 millimetres to the tube-length, and in general it may be taken that no changer adds less than this. The only satisfactory way is to ascertain when purchasing any such appliance the actual allowance that it entails.

In practice it is desirable to prevent as far as possible any dust or dirt from reaching the objectives, and when one is removed from its case, both the box portion and the lid of the case should be inverted on the table to prevent the entry of dust. A well-washed piece of linen or silk should be kept specially for the purpose of cleaning an objective. It is advisable to keep a small piece in a stoppered bottle or some such closed receptacle, and only to take it out as required, replacing it immediately after use. To clean immersion objectives a very slight moistening of the rag with zylol or benzole may be recommended. Any excess of either of these solvents is to be carefully guarded against, as it may injure the objective if it reaches the inside of the lens mount. Possibly a better material for cleaning purposes in general, however, is Japanese silk paper ; a small piece of this may be torn off when required, and can then be thrown away after use, so that no dirt can accumulate on it. 
For observational work the selection of the most suitable ocular, and the advisability of using low-or high-power ones, is an important matter; but in photo-micrography any but lowpower oculars may, except under very special circumstances, be excluded. On occasions it may be necessary, where great amplification is required, to use one of fairly high power; but this will only be found to work satisfactorily when the objectives are apochromats, and the oculars therefore of the compensating type. If an ocular other than of the projection type is used, no adjustment of its parts is necessary when a photograph is being taken, but with the projection-oculars it is important to see that the image of the diaphragm inside the ocular itself is quite sharply projected on the focussingscreen of the camera. This procedure has been sufficiently described under the section giving the various types of oculars. It is essential to ensure that, in the case where no ocular is used in conjunction with the objective, the inside of the bodytube of the microscope is completely blackened, as otherwise internal reflections are sure to result, and a central flare-spot will appear on the focussing-screen of the camera. This is most effectively provided against by having a little cardboard tube which is lined with black velvet, and which is of such a size that it slips into the body-tube; it should be of such a length that it comes quite flush with the top of the latter.

Achromatic objectives that may. give an extremely good visual image are often not corrected to give a sharp photograph in the same plane; in fact, generally the better the visual image, the less likely is it that the photograph will come out sharp. The reason of this is that achromatic lenses are corrected for the yellow and green rays only-that is, for the portion of the spectrum of the greatest visual luminosity ; but they are not corrected to utilise in the formation of the image the less luminous blue and violet rays. With apochromatic objectives this does not apply, and it will be found that these give a perfectly defined image whatever the illumination may be. One method of overcoming the difficulty with achromatic objectives is to ascertain the difference between the distances of the visual focus and the actinic focus from the microscope stage. This can only be effected by taking a series of photographs with each lens, and carefully measuring the alteration 
in the focus that has to be effected to secure a sharp photograph. If the fine-adjustment on the microscope is a graduated one, then this is not a matter of such great difficulty; it is only necessary to determine the number of divisions through which the micrometer screw has to be moved to obtain correct photographic focus, and to alter the fine-adjustment by this amount every time when a photograph is to be taken. It is, of course, assumed that the object is visually focussed beforehand on the ground-glass screen of the camera, and then this allowance made. Obviously this is a somewhat laborious matter, and it is by no means certain that under all conditions the focussing will prove to be as good as might be desired.

An alternative and far better method is to use invariably a colour-screen with achromatic objectives. This does not very substantially increase the exposure, as it only requires to be of a pale-yellow or pale-green colour-one that will effectively cut out the blue and violet rays. Or again, if the screen is approximately monochromatic, whatever colour it may be, the object when focussed visually will nearly always be found in correct focus photographically. The best screens to use for this purpose will be given in Chap. IX of this book, which treats of the use of colour-screens in general. It will be obvious that where possible it is better to obtain apochromatic objectives, since these colour differences do not then arise; although it must be admitted that where the necessary trouble has been taken to secure a correct colour-screen the difference between the results obtained by using apochromatic or achromatic objectives can often hardly be appreciated.

It is important to remember that dust on either of the components of the ocular will become painfully evident in the photograph. For this reason it is almost more necessary to ensure that the oculars are kept perfectly free from any dirt than any other optical part. If dust is evident in the field of view, it can at once be determined whether it is on the ocular or in the object, by rotating the ocular itself ; then, if it is on any part of the latter, the image will move round. Steps may then be taken to remove it, but the cleaning should be as carefully performed as in the case of an objective. If it is necessary to unscrew any part, it is essential to see that on replacing it is firmly screwed up again to its correct position. 
In using illuminants of high power, under no circumstances should the attempt be made to observe directly the image in the microscope, unless some dark glass or similar light modifier is placed in any convenient position between the illuminant and the eye. The advantage of the method of carrying out the various adjustments by means of the projected image on an opaque screen becomes further evident in this case, as there is then no possible risk of any harm to the eyes. This is a point that requires careful consideration when working with illuminants of the type of the electric arc, or the quartz mercuryvapour lamp. From both of these a very considerable emission of ultra-violet light takes place which, even with an exposure of a minute or two, will cause very painful and even serious inflammation to the eyes. They should in all cases be efficiently screened off so that no direct light reaches the observer.

Correction for Thickness of Cover-glass and Adjustment of Tube-length.-A point of great importance in using high powers-particularly those other than immersion objectivesand one which affects the quality of the image to a very marked degree, is the thickness of the cover-glass over the object. A flat refracting surface behaves to some extent like a spherical one; in fact, the flat surface may in theory be regarded as a spherical surface of infinite radius of curvature. It therefore suffers, although to a less extent, from the same defect as such a curved surface; oblique rays are refracted to a greater extent than those situated more centrally. If an object is mounted in Canada balsam, it results that light emitted from any one point in the object, when it emerges from the surface of the cover-glass into air, would proceed not as if it were coming from one point, but from a succession of superimposed points, and the effect would therefore be that the rays of greater obliquity appear to emerge from a different point from those that were less oblique. Fig. 55 diagrammatically illustrates this point. Let the horizontal line FG represent the top surface of a coverglass, the object being mounted in Canada balsam beneath it. If light were being emitted from a point in the object $A$, it would appear not as if it were from A only, but also as if it were emitted from the points B, C, or D, according to its obliquity. This example is, of course, hypothetical and exaggerates the actual conditions for purposes of demonstration. The practical 
effect is, however, that a cover-glass introduces an aberration which the objective has to correct. If the cover-glass is thicker or thinner than the particular thickness for which the lens is corrected, it would therefore influence the performance of the lens; the latter would not then give as good an image as when working under proper conditions. In the case of apochromatic lenses, particularly the 4-mm., and usually in the best quality achromatic lenses of about one-sixth inch, provision is made, by means of what is called a correction-collar, for altering the distance between some of the component lenses of the objective to compensate for variation in cover-glass thickness. Lenses

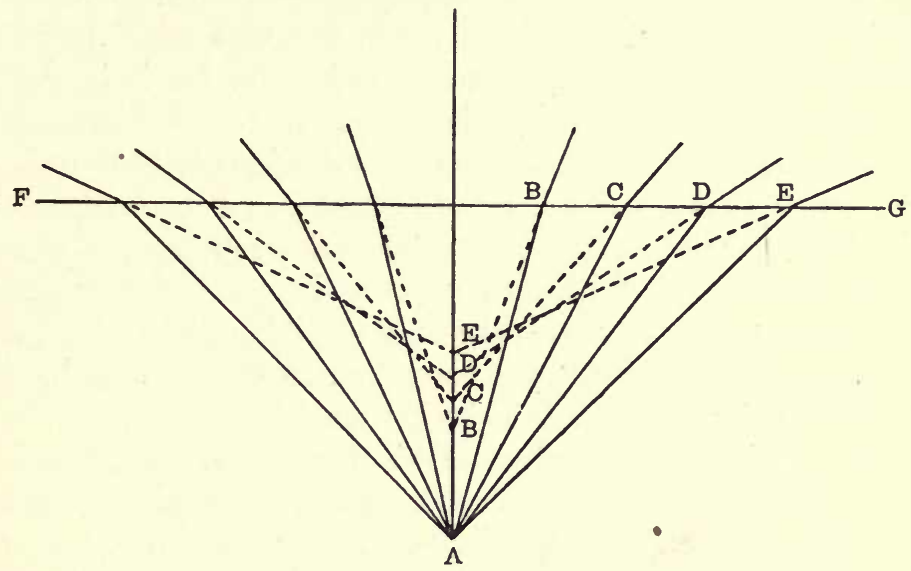

Frg. 55.-Influence of Cover-glass Thickness on Adjustment.

are now usually corrected for a thickness of cover-glass of $0 \cdot 17$ to $0.18 \mathrm{~mm}$., and any variation from this thickness introduces an error which is comparable to spherical aberration. To obtain the best results with any lenses, a cover-glass of the exact thickness for which the lens is made, and which the makers usually indicate in their catalogue or on the lens mount, should be used. This, however, is rarely possible, so that adjustment has to be made by the correction-collar provided on the objective itself. An alternative method when such a correction-collar is not provided, is to alter the tube-length-that is, the distance between the objective and the ocular.

Qil-immersion objectives do not require any adjustment 
for this variation in thickness when the object is mounted in a medium of approximately the same refractive index as glass, for under these conditions no refraction of the light occurs at the surface of the cover-glass. When work of the most critical nature is being carried out, particularly when the resolution of the structure of diatoms mounted in media of high refractive index is attempted, this does not apply. The correction necessary in these cases, however, is usually very small and such that it may be effected by alteration of tube-length; in this class of object great care is exercised and considerable experience is necessary in making a preparation, and it is usual in such cases to calibrate carefully the cover-glass before mounting up. It is partly for this reason that the unskilled microscopist so generally prefers an oil-immersion objective, for it is not so sensitive to any small variation from the required conditions as a dry lens. The advantage of the dry lens is that it is of greater focal length, and if the magnification required can be obtained with it, then the additional working distance is often a great advantage. With some objects where a thick cover-glass has been used a dry lens is the only possible one to adopt, and in such cases the necessary correction must be made by the correction-collar, or the tube-length must be shortened.

The adjustment by observation of a lens with a correctioncollar is not a very easy matter except to a microscopist of considerable skill and experience. It is obviously impossible to measure accurately the thickness of a cover-glass on a mounted object; but where a microscope possesses a graduated milled head to its fine-adjustment, an approximate estimate of its thickness may be obtained without much trouble. If the objective in use also has marked on it the correct position of the index on the correction-collar for a given thickness, as it should have, it is then only necessary to set the collar for the determined thickness. If the specimen should be an ordinary bacteriological cover-glass preparation, all that is required is to focus a bacterium in the preparation, and then to raise the objective by the fine-adjustment until some very small particle of dust on the top surface of the cover-glass comes into view. The difference of the focus, as shown by the graduation of the milled head, will then indicate the thickness of cover-glass in use. To ascertain the thickness of the glass, however, the 
distance so found would require to be multiplied by the refractive index of the glass (approximately $\frac{3}{2}$ ), and this would give the approximate thickness of the cover. It is, however, clearly of the nature of a makeshift method, since no allowance is made for possible variations in the thickness of different parts of the same cover-glass - a factor by no means negligible - or for the cases in which the part of the object in view lies a greater or less distance below the cover-glass. It is not nearly so exact or so satisfactory in any way as direct observation of the effect that collar-adjustment or alteration of tube-length has on the image itself.

Reference to Fig. $56 a$ and $b$ will enable a better idea to be

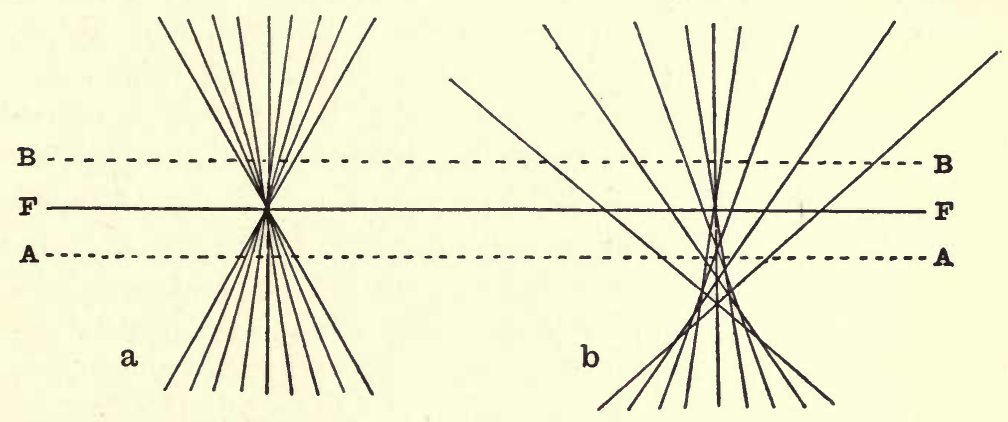

FiG. 56.--Influence of Cover-glass Thickness on Adjustment.

obtained of the manner in which this is to be effected. Fig. $56 a$ shows the path taken by rays of light which come from a perfectly corrected lens system, and which form an image so that all the rays pass through one point. Fig. $56 \mathrm{~b}$ shows the image formed by a lens system that is not in correct adjustment, and in which the rays do not come to one point. It is evident from Fig. $56 a$ that if the rays are examined at the position $A$ or $B$, instead of at the point $F$, although the object will be out of focus the images given will be identical whether we examine them on the one side or the other of the correct focus. In either case a disc of light will be seen, and these dises will be equal in brilliancy; the dise $\mathrm{A}$, in fact, will be exactly the same in every particular as the disc B. Such, however, is not the case with Fig. $56 \mathrm{~b}$. The image in the position $\mathrm{A}$ will here be very much more brilliant than in the 
position $\mathrm{B}$; in fact, at $\mathrm{B}$ there will be nothing but a large and diffused patch of light. The images observed at the points $\mathrm{A}$ and $\mathrm{B}$ in this case will therefore be totally dissimilar. This is the method actually adopted in correcting for a desired tube-length or thickness of cover-glass, and opticians when effecting this correction in the course of manufacture usually have some object with which they are familiar and of which they know the appearance under varying conditions. On focussing such an image first above and then below the focus, and successively performing this operation and comparing the two appearances, it can be determined whether the objective is properly corrected for the conditions under which it is to be used. It must at once be said that it is not by any means easy for a novice to appreciate the differences, and to get correct adjustment; a good deal of practice is necessary, and in fact only the trained microscopist can obtain the best correction in actual practice. When working with an object which has a cover-glass of unknown thickness it will be found best to focus and to effect the correction by means of some small dust-particle in the object. On rapidly focussing this, both above and below its proper focal point, the difference in the images will become apparent. The objective must then be corrected by its correction-collar, if it has one, or by altering the tube-length of the microscope until its image is such that it appears exactly the same whether the focussing is above or below the proper focal point. Alternatively, any fine line or hair in the object may be utilised to perform the operation. If the lens is not properly adjusted, the appearances on opposite sides of the true focus will not be identical.

The best object for demonstrating the effect is a Podura (Lepidocyrtus curvicollis) scale. This is the one most universally employed by opticians to correct their objectives both for thickness of cover-glass and for length of tube. Fig. 57 shows at $a$ the appearance of a portion of the scale that may be expected if it is in correct focus and the lens perfectly adjusted; at $b$, the appearances well outside both sides of the focus when the adjustments are correct, and at $c$ the appearance as it is just going out of focus on either side ; $d$ and $\boldsymbol{e}$ show the two distinct appearances, one on either side of the focus, when the objective is not in adjustment ; $f$ shows the 

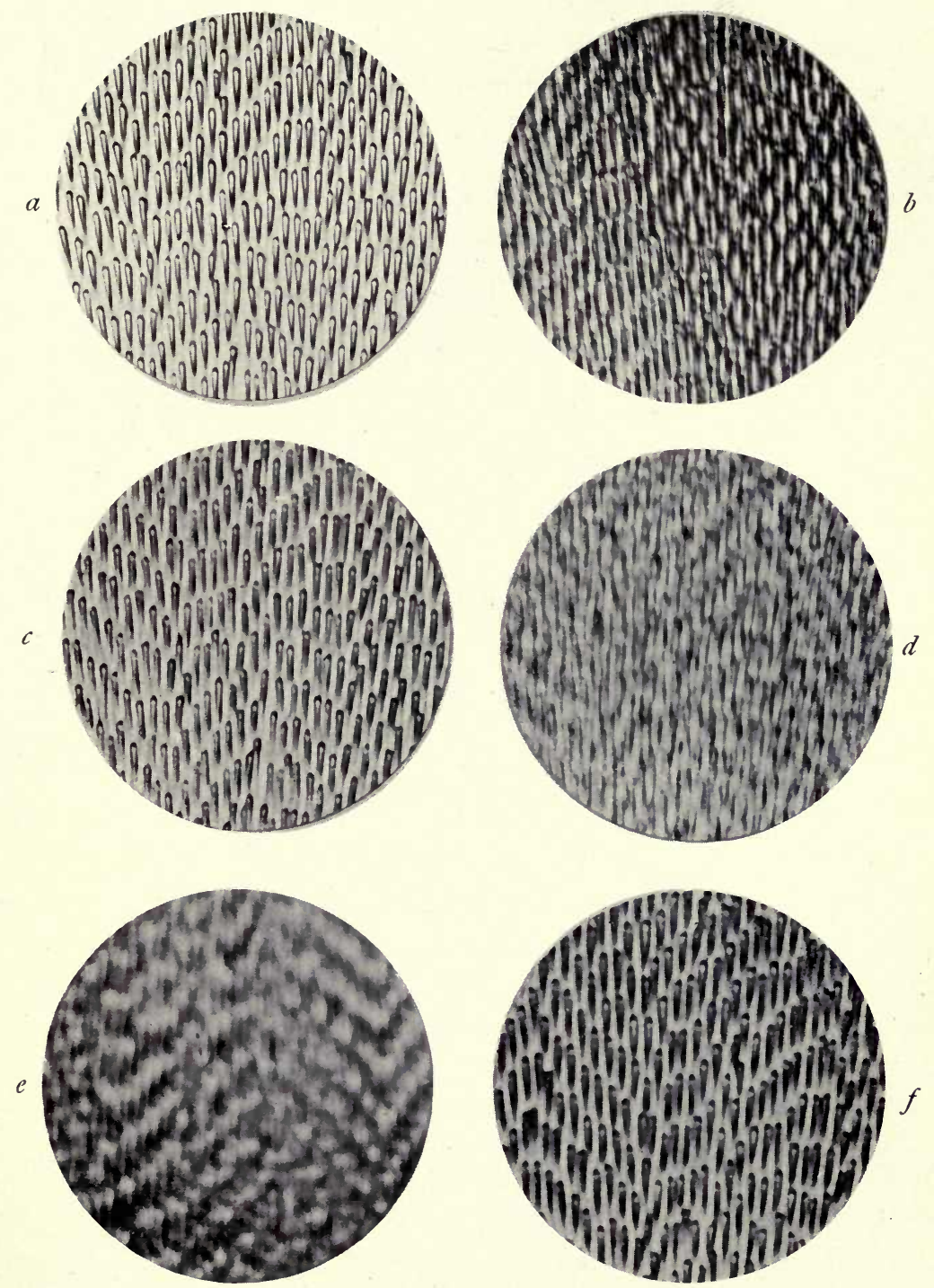

F1G. 57.-Effect of varying Cover-glass Thickness on a Podura Scale. 

nearest approach to a sharp image that can be obtained with a lens not properly corrected. ${ }^{1}$

The reason why the Podura scale is such a good test, is that the appearances on either side of the focus when the lens is not properly corrected are of different patterns ; when the lens is in correction, this is changed into one and the same pattern whether the lens is moved either within or without the focus.

It is unfortunate that at present good mounted Podura scales are difficult to obtain, and even those that can be got are expensive. An alternative is the well-known diatom Pleurosigma angulatum. This object requires a good deal of practice to get the best appearance under even proper conditions, and in some respects it is perhaps somewhat misleading, as the appearances to be obtained are so very varied. Select one mounted dry and with a frustule that is in optical contact with the cover-glass ; if the frustule is itself fractured so that a broken edge is in the field of view it will facilitate correction. Carefully focus this broken edge so that it shows what is usually referred to as the postage-stamp fracture. If the correction for cover-glass thickness and for tube-length is as it should be, then on getting this edge exactly into focus the slightest possible movement of the fine-adjustment will bring into view a black-dot pattern which should be perfectly defined. If either of the conditions named are departed from, this blackdot pattern will only appear if the fine-adjustment is moved to a considerable extent, either above or below the point at which the broken edge is in focus. If it is necessary to focus downwards to get the black-dot image into view, it indicates that the tube-length must be increased; or alternatively that the correction-collar on the objective must be altered in the direction which would be required when the thickness of the cover-glass is less than that for which the objective is corrected. On the contrary, if it is necessary to focus upwards, the opposite conditions are indicated; and in each case the tube-length or the adjustment by the correction-collar must be altered in the contrary direction. Perhaps for a beginner this object is even more useful than a Podura scale, for even in cases where there is but a brief experience of the appearance of microscopic

1 See Cantor Lectures on The Theory of the Microscope, by Conrad Beck. 
images, the position of the image either above or below a particular focal point is quite easy to observe. It will be of educational value to watch the effect on other parts of the image, varying the two conditions referred to, as it will facilitate these adjustments being made on an object that does not lend itself to this particular treatment.

It should be noted that in all cases where such corrections are to be made the highest power eye-piece that the objective will stand should be used. In the case of achromats this will therefore be an eye-piece that does not enlarge the image more than ten times, while with the apochromatic objectives an ocular magnifying eighteen times is the most convenient one to employ.

It is far preferable, where possible, to ensure that the object is covered by a cover-glass of the correct thickness, and to use the lens at the correct tube-length as indicated by the maker. The method of correcting for thickness of cover-glass by alteration of the tube-length is not so satisfactory ; in fact, when using medium or low powers, some variation from the correct tube-length is not so serious under ordinary conditions of work. It has been found by experiment that an alteration of one-thousandth of an inch in the thickness of the cover-glass when using a particular high-power dry lens is equivalent to an alteration of seventeen inches in the length of the draw-tube. It is not advisable to infer from this that the length of the tube has a negligible influence on the correction, although its effect is in general somewhat exaggerated. Its importance becomes much more evident when lenses over one-sixth of an inch are used. The correct length of tube may be determined by observing the image in the same manner as that already described for correcting for thickness of cover-glass. The appearance of the image is exactly the same when there is any substantial variation from the correct tube-length as in the case where the correct thickness of cover-glass is not being used.

The Use and Adjustment of the Sub-stage Condenser.When using objectives of two-thirds or a half inch, as well as higher powers, a sub-stage condenser must be employed. This should preferably be of the achromatic variety, and should be carried in a centreing sub-stage together with an 
iris-diaphragm. It is perhaps unfortunate that the majority of cheap microscopes, particularly of the Continental pattern, are not supplied with any arrangement for centreing the substage condenser. High-power work, therefore, if of even an approximately critical nature, becomes somewhat difficult when such an instrument is used. Various types of sub-stage condensers can be obtained and have already been described, so that it only remains to indicate their methods of use.

The aperture of the condenser adopted should approximate to, if not equal, that of the objective of highest aperture in use. It will usually be necessary to close the iris-diaphragm to some extent to obtain satisfactory illumination; but unless the condenser-aperture nearly equals that of the objective, the full advantage of the latter cannot be obtained when the resolution of difficult diatoms or similar objects is attempted. Most achromatic condensers may be used alternatively with medium or high powers if the top hemispherical lens is removed, as it usually can be ; the remaining portion then forms a very satisfactory condenser, which can be used with medium powers. It is obvious that, to utilise the whole of the aperture of immersion-objectives, the sub-stage condenser must itself be an immersion one; but some of these also work fairly satisfactorily if used dry.

In illuminating an object the first point is to ensure that the optical centre of the condenser coincides exactly with that of the objective. This may be determined by closing the iris-diaphragm as much as possible so that only a very narrow beam of light passes through. The objective should of course be upon the microscope, and some simple object in focus. Rack down the condenser until the image of the aperture in the closed iris-diaphragm comes into view and is sharply focussed ; it will then appear more or less well centred in the field of view. By the aid of the centreing-screws on the sub-stage bring this image of the diaphragm exactly into the centre of the field. It is advisable to slowly open the diaphragm until this image reaches the edge of the field. It should be so adjusted that it disappears from view evenly all round the visible field. It is essential that the illuminant should be approximately centred before the adjustment is made, as otherwise it is not easy to get the whole of the outline of the 
diaphragm-aperture in correct focus ; in fact, if there is any falling off or haziness to one side or the other, it clearly indicates that the illuminant is not in its correct position. This preliminary operation will usually be carried out with the microscope on a table, and suitably inclined for visual work. The centration of the light is therefore carried out by moving the mirror until the desired effect is obtained. This adjustment having been made, the condenser may now be racked up until the image of the radiant comes into view, and this image should be as carefully focussed as possible if critical illumination is desired. The diaphragm may now be opened, and the degree to which this should be done or not is a matter of considerable moment. The merest novice will soon perceive that, with a diaphragm that is closed to a considerable extent relatively to the aperture of the objective, a very great falling off in the quality of the image results. For ordinary purposes, and when observing pathological, botanical, or any similar preparations, the best result is usually to be obtained when the diaphragm is open sufficiently to fill approximately two-thirds of the objective-aperture. The proportion of the objective-aperture that is being utilised may be determined by removing the ocular from the body-tube of the microscope and looking down the tube. If the whole aperture is being employed, the back of the objective will appear to be quite filled with light, whereas when the diaphragm is closed the circle of illumination gradually decreases. The most satisfactory result is usually obtained when the diameter of the circle of illumination is equal to two-thirds of the diameter of the whole visible aperture of the back lens.

There are very few objectives which will, under ordinary circumstances, stand a larger cone of illumination than this; but for bacteriological or similar preparations it will be found that a larger cone may safely be used. In photographing it is, from a pictorial as well as from other points of view, necessary to have an evenly illuminated field. This may often be obtained by racking up or down the condenser, and so departing from the conditions that give critical illumination. This, therefore, is a method of dealing with the matter that should never be adopted if any other means of attaining the same result are available. The object of the auxiliary collecting-lens is to get 
over this difficulty, as in effect they enlarge the image of the source of light. If for any reason the only method of getting even illumination is by altering the position of the condenserthat is, by departing from the conditions necessary to obtain critical illumination-then it would appear to be better for the condenser to be raised nearer the object-slide, so that the apex of the cone of illumination is above the object, rather than for the reverse operation to be performed.

It should be remembered that sub-stage condensers are always of very short focus, and it is therefore advisable that in making these adjustments only medium-power objectives should be on the microscope, so that in the event of the condenser being raised too high and coming into contact with the underside of the object-slide, the latter is not forced up against the front lens of the objective. It may sometimes happen with a very thick slide that it is impossible to focus correctly on the object, and in this case, of course, critical illumination is unobtainable. This condition may be to some slight extent overcome by bringing the illuminant nearer the condenser; but, obviously, such a method is but a makeshift and is a remedy which can only be effectual within narrow limits.

It must not be overlooked that when using any form of objective-changer - such as a multiple nose-piece-the substage condenser must be re-centred for each change of objective. It very rarely happens that these appliances are sufficiently correctly made to ensure the optical axis of a series of objectives always coming in the same position. With the Zeiss objectivechanger or any similar appliance this does not apply, as it is usual for the worker to centre carefully the whole series of objectives in use when the changers are fitted to the objective.

In centreing, where a change of objective becomes necessary, the following method is satisfactory. Place on the microscope the objective with which the photograph is to be taken, presumably a high-power one. The object on the stage may conveniently be a glass slip on which two diamond lines or scratches at right angles have been made, as already described, this being the most convenient object to use for all preliminary centreing purposes. Arrange this object on the stage so that the point of intersection of the diamond scratches appears exactly in the centre of the field. Now remove the high-power objective 
and substitute for it one of medium power, and carry out the necessary operations to obtain correct centration, as already described; but, in this case, the illuminant and condenser must be centrated not to the centre of the visible field of the objective, but to the point where the images of the diamond lines intersect, since this is the position of the optical centre of the objective to be used. The centration will therefore be correct when the high-power objective is replaced with which the photograph is to be taken. Assuming that the illuminant and any auxiliary condensing-lens have been carefully centred for one objective, it is usually not necessary to alter the position of these when effecting a change of objectives; or if any movement is necessary it is very slight. The differences of centration in the latter are usually not so great as to render it necessary to alter the position of the illuminant, re-centration of the sub-stage condenser being sufficient to effect the desired result.

It is assumed that the adjustments so far described have been made, or at least that there has been some preliminary practice of the process with a microscope on a bench and not on the photo-micrographic apparatus. The centration of the object and the sub-stage condenser having been effected, it remains to place the microscope in a horizontal position on the photographic apparatus, and the illuminant in position at a suitable distance from the microscope. If no auxiliary condenser or bull's-eye is used, this distance will be about ten inches. It will then probably be found that the image of the illuminant is not in the centre of the field. To watch this image through the microscope and alter the position of the illuminant so that it is exactly central is usually a somewhat troublesome process, as it is generally difficult to reach the source of light while observing the microscopic image. It is assumed that the microscope is carried on a revolving table, so that it can be thrown out of the optical axis or that the camera itself may be moved back to allow of direct observation of the image. If the apparatus is of the former type, allowing of the movement of the microscope and illuminant as a whole, the great advantage of the small subsidiary opaque screen, set at the side of the focussing-screen of the camera as already described, will now become apparent. It makes it possible 
to project the image on to this opaque screen, and exactly centre the image of the illuminant to the centre of the field. In fact, given a sufficiently powerful source of light, it is by far the best method to make all the adjustments, centreing the condenser and the illuminant, getting the correct position of the object in the field, and closing the iris-diaphragm to the required amount, by observing the projected image. An illuminant of great power is not necessary for this purpose. Among the electrical ones, a one-ampere Nernst lamp on a 240 -volt circuit gives quite sufficient light to make the necessary adjustments even with oil-immersion objectives. A small are lamp-such as the Leitz form-taking four amperes, will enable adjustments to be effected if an object is being magnified as much as two thousand diameters. It is obviously easier to exactly centre the optical components and the illuminant with a large image projected to a distance, and with the operator free to adjust any of them with facility, than to do it while observing the image in the microscope.

To prevent confusion it may be wise to recapitulate here the exact order in which the operations described should be performed :-

1. Set up and centre the object with the objective with which the photograph is to be taken.

2. Remove this objective, if it is a high-power one, and substitute for it a low or medium power.

3. Close the iris-diaphragm in the sub-stage condenser as far as possible.

4. Rack down the sub-stage until a clearly defined image of the opening in the iris-diaphragm is seen in the field.

5. Centre this image of the iris-diaphragm until it is in the centre of the field ; or alternatively, where a change of objective is necessary, until the image is exactly projected over the point of intersection of the diamond lines on the slide already described.

6. Rack up the condenser until an image of the illuminant is exactly focussed in the field of view, the object itself also being in correct focus.

7. Centre the image of the illuminant by moving the source of light so that it falls exactly across the microscopic image.

In cases where the illuminant is of large area, and where 
therefore its image more than fills the field, a good plan is to place a metal plate with a small hole in it as near as possible to the illuminant, so that only the centre of the latter is exposed to view. Then centre the image of the aperture in the plate-this, in fact, being treated as the source of light. This will only be necessary when a large-flame oil lamp is used, as no other illuminant is of sufficiently large area to more than fill the field when using low- or medium-power objectives.

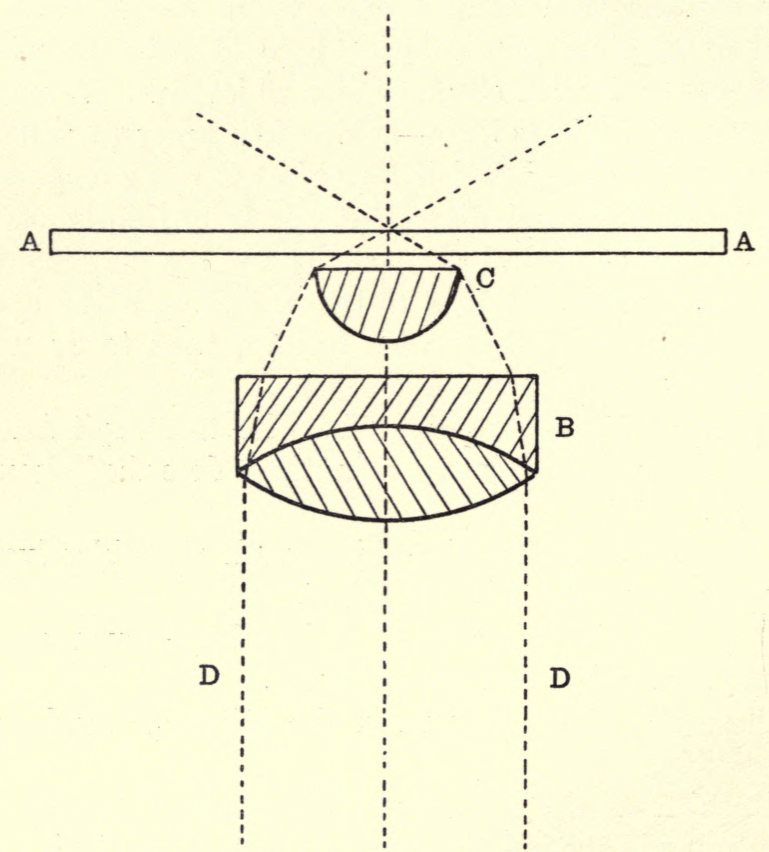

Fra. 58.-Diagram of Path of Rays in Sub-stage Condenser.

All these adjustments are best effected with a low-power ocular. The operations are similar whether the whole of the sub-stage condenser is used-as in the case of high-power objectives-or only the back combination. The advantage of using a well-achromatised condenser and one having a large aplanatic cone will, during these operations, become evident. The image of the radiant should appear bright and well defined in the field, and that portion of the field that is not covered by the radiant should appear relatively dark.

Fig. 58 shows diagrammatically the condition that should 
obtain when the condenser is filled with a parallel beam $\mathrm{DD}$, and is exactly focussed on the object lying on the slip AA. If the condenser is one with a full aplanatic cone-that is, one which is practically free from spherical aberration-the whole, or nearly the whole, of the light will be focussed on the object.

In the case where the condenser is simply an uncorrected lens or is of the ordinary Abbe illuminator type, then the central rays will be focussed in a different plane to the marginal rays (Fig 59); and to get a clearly defined bright image of the radiant it will be necessary to close the irisdiaphragm somewhat, irrespective of what may be necessary to obtain a bright image of the object.

Not only is it impossible to obtain such a bright and clear image of the illuminant, but there is also a general loss of light owing to so much of the aperture of the condenser being used in condensing the light to a point where it is not utilisable by the objective. It must also be remembered that unless critical illumination is accurately obtained, the effective aperture of the condenser itself is reduced; that is, if the condenser is adjusted so that its focal point is either above or below the object, the effective aperture-as may be observed by looking down the microscope tube without the ocular-is less in proportion as the correct conditions are departed from. Fig. 60

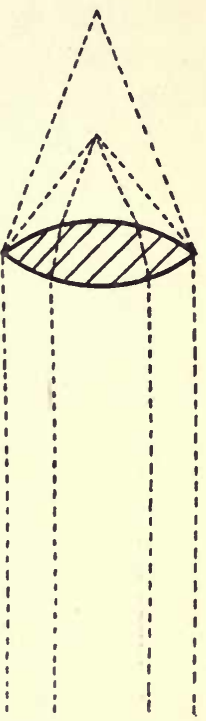

Fia. 59.-Diagram of Path of Rays in Sub - stage Condenser. may help to explain this. The points A, B, and $\mathrm{C}$ may be taken to represent the position of an object in the cone of light formed by the condenser. Only at A does the object receive the benefit of the full cone, and at $\mathrm{B}$ or $\mathrm{C}$ neither the full amount of light nor the full aperture of the condenser is utilised.

A further advantage of the large aplanatic cone becomes evident when test-diatoms or similar objects have to be photographed with only a portion of the solid cone-that is, with annular illumination. In this case only a portion of the marginal rays transmitted by the condenser is utilised. The 
object therefore would not be critically illuminated by a condenser having a small aplanatic cone if a stop with an annular opening were inserted; and assuming that the object had been previously illuminated with a solid cone some further adjustment would be required. In such cases a condenser of the highest possible order is essential : it must be well achromatised, and be

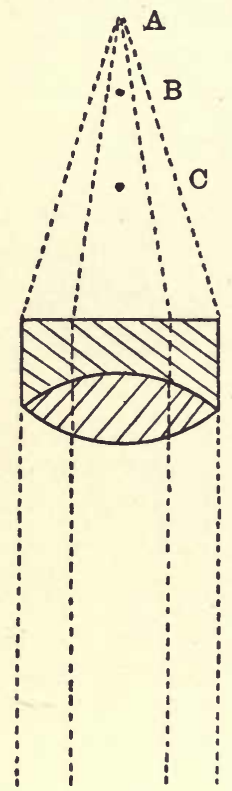

Fra. 60.-Diagram of Path of Rays in Sub - stage Condenser. as free as possible from spherical aberration. The shapes of the various stops that may be used have been already indicated, but in general it will be found that it is more convenient to improvise stops from thin black cardboard, cutting these to the shape and size required to bring out the details of a particular object.

For work of the most critical nature, some workers are of the opinion that any auxiliary condensing-lens between the illuminant and sub-stage condenser is to be avoided if possible. In such cases it may be better to use an illuminant of somewhat greater area, to place it at its proper distance from the sub-stage condenser-usually a distance of approximately ten inches-and to focus the image of the radiant without any intervening optical system. The next best arrangement is to use an achromatic auxiliary collecting-lens and to project a parallel beam from a very small source of light, such as the electric arc, into the back combination of the sub-stage condenser. The writer has yet to find an object that cannot be perfectly illuminated by this method. Among high-power illuminants for highly critical work, lime-light has the advantage that it admits of the direct projection of the image of the radiant in the objectplane without any intervening optical system, since it is both sufficiently powerful and of large enough area. The same may be said of the mercury-vapour lamp, although its intrinsic brilliancy does not approach that of an efficient limelight jet.

The Auxiliary Collecting-lens. - The advisability of the use of such a lens or lenses is a somewhat controversial point, and 
some workers of repute are of the opinion that no optical system should intervene between the source of light and the sub-stage condenser of the microscope. While for observational purposes this may hold, it becomes a matter of extreme difficulty to do without some such appliance in photographic work. If for no other reason, the pictorial effect would be so much interfered with unless the field is evenly illuminated, that some method of obtaining this desired result becomes almost essential. The necessity for this is more particularly in evidence with a source of light of small area-such as the electric arc, or electrical illuminants of the Nernst type. With lime-light, acetylene, or the oil lamp, it is quite possible to work with moderate or high powers without an intervening lens system; but in any case the exposure is lengthened, and it is by no means clear that any deterioration of the image results from its use.

For very low-power work the auxiliary condenser may be replaced by a piece of ground-glass, and an enlarged image of the illuminant may be projected on to this; the ground-glass in effect therefore becomes the source of light. The conditions with higher powers are that either the image of the source of light is enlarged by using an auxiliary lens so that it covers a larger proportion of the field, or the position of the radiant is so adjusted that the aperture of the lens itself is what is actually focussed in the field of view-the image therefore in the field of view appearing as a luminous disc.

Perhaps the most serious practical objection, and one that requires to be carefully guarded against, is that any intervening lens, unless exactly and properly adjusted, may, under certain circumstances, reduce the working aperture of the objective; and it is against this contingency more than any other that the worker is warned.

The simplest lens of this type is of course the bull's-eye, of which a description has already been given, and which is only suitable for use with low powers. A development of the bull'seye system is the more highly corrected lens system devised by Mr. E. M. Nelson, and usually known by his name. This lens has already been described under the section devoted to such apparatus in Chap. IV, p. 70, as well as the WatsonConrady system, which is still better and the corrections of which satisfy the requirements of the most critical worker. 
In actual use all these lens systems are subject to practically the same working conditions, and it is therefore proposed to describe the method of adjusting the complete lens system already described as made by Messrs. Zeiss ; these meet almost every need, are comparatively inexpensive, and are available for use with powers from the lowest to the highest.

In conjunction with these lenses an iris-diaphragm on a separate stand is usually supplied, and in any case, whatever form of collecting-lens is used, such an addition is almost a necessity. A less expensive substitute is a series of metal plates, with holes of various sizes in them, which may be supported in any suitable carrier and which are so arranged that they can be easily changed as required. The first point is to ensure that this iris-diaphragm or its equivalent is accurately centred to the optical axis of the microscope, when the latter is in the horizontal position, and is in alignment with the centre of the camera back. To be quite certain that this is the case, it must be observed in relation to the sub-stage iris on the microscope. In some instruments there are means of de-centreing this iris, the rack-work that enables the de-centration to be effected having a catch which indicates to the worker when centration is correct. With the usual English form centration of the iris would be effected by means of the centreing-screws on the sub-stage. The operation may be performed by placing a low-power objective in the microscope, inserting an ocular, and viewing the image of the diaphragm directly. Any necessary alteration in the adjustment may then be accomplished by means of the screws on the sub-stage already mentioned. The ocular is next removed, and in its place a cap, which is perforated at its centre with a small hole less than one millimetre in diameter, is substituted. On observing the source of light through this eye-piece cap and the closed iris-diaphragm of the microscope sub-stage, it will at once be seen whether the illuminant is in correct alignment, as unless it is, no light will pass through to the eye. The illuminant having been adjusted centrally, the subsidiary diaphragm first mentioned may now be interposed between the sub-stage of the microscope and the illuminant. It facilitates any future adjustment if these extra fittings are carried on a triangular metal bar or geometric slide, as already described; 
but in any case, they should be so arranged that they may be removed and replaced in their proper position at will. The iris-diaphragm should now be closed as far as possible, and if all is in correct alignment on viewing the illuminant through the eye-piece cap, the closed sub-stage iris, and the extra intervening diaphragm, a brilliant point of light should be seen. It is obvious that if there is any want of centration between these three small apertures, no light can pass, and it will be found extremely useful to devote some attention to this point at the start to ensure that such adjustment is exactly carried out.

This having been done, the auxiliary condenser may now be placed in its position, which should be between the illuminant and the auxiliary iris-diaphragm. Assuming that this collecting-lens is the one described by Messrs. Zeiss by the Roman figure I, the iris-diaphragm should then be placed near to it. If reference is now made to Fig. $21 a$, it will be seen that when using this No. I lens a convergent beam is thrown into the back lens of the sub-stage condenser, and this should be a sufficiently broad beam to fill completely the back lens of the sub-stage condenser with light. This lens is of the uncorrected plano-convex type, and the same adjustment may therefore be made when using a bull's-eye or any other similar system.

An alternative method when a parallel or slightly convergent beam is being used, is to place the subsidiary irisdiaphragm as near as possible to the collecting-lens, close the iris and focus its image by means of the sub-stage condenser in the field of view ; the iris of course is opened again sufficiently to fill the field of view when a photograph is to be taken. A critical image of the iris is therefore obtained instead of a critical image of the illuminant. It must be admitted that while this does not exactly conform to the methods advocated by some authorities, it does in practice give extremely good results. For high powers this lens No. I is sufficient to obtain a well and evenly illuminated field, even with an illuminant of the smallest area, such as an electric are of small current-consumption.

For medium powers the arrangement is seen in Fig. $21 b$. In this the No. I lens is taken much closer to the source of 
light, so that a parallel beam is projected. The lens No. II is then placed in such a position that it takes up this parallel beam and focusses a rough image of the source of light on to the iris-diaphragm of the sub-stage condenser of the microscope. In this case the auxiliary iris-diaphragm occupies a position between the two condensing-lenses, and is therefore controlling the diameter of the parallel beam. The image of the radiant as seen at the back of the sub-stage of the microscope is also much smaller; but, as with medium-power objectives the aperture of the sub-stage condenser is of necessity less, or at least its effective working aperture is less, the projected image is sufficiently large to fill the field and does so without any reduction of aperture. In this case again the image of the closed auxiliary iris may be focussed in the field of view.

Reference to Fig. $21 c$ will show the arrangement for low powers. It does not differ in principle from the preceding arrangement, except that the lens No. III, of shorter focus than No. II, is substituted. The lens No. III is in fact similar to No. I, but, instead of having its plane side towards the illuminant, is placed with its concave side in that direction, so that it throws a small image of the source of light on to the sub-stage iris-diaphragm. The same conditions are here again to be complied with as in the preceding example, and the image of the auxiliary iris is once more focussed in the field of view. It is assumed in the latter case that the sub-stage condenser is one of large aperture, and probably will be one that is used for higher power work, but has had its top combination removed. In this case it will be found that the working aperture of the condenser is, of course, again reduced; but this is not a matter of moment, as the objectives to be used in conjunction with it are themselves of proportionately smaller aperture. These arrangements are to some extent influenced by the illuminant in use. For instance, the combination of Nos. I and III condensers, if used in conjunction with a Welsbach inverted incandescent light, will answer perfectly for medium as well as for low powers, so that the conditions may be altered somewhat according to the size of the illuminant that is in use. Again, the arrangement of the auxiliary condensers is to some extent dependent on the sub-stage condenser, as on its focal length depends to a great extent the size of the image of the illuminant 
that is projected into the field of view. Variation within some limits is therefore possible, depending on the particular substage condenser.

Whatever auxiliary condensers are adopted, it is most important to see that they are so adjusted that no reduction in the available working aperture of the sub-stage condenser results from their use. This may be determined by first obtaining a critical image, in the manner already described, without any optical system intervening between the source of light and the microscope. Then remove the ocular and see, by looking at the back lens of the objective in the manner also previously described, what proportion of the objective-aperture is actually being used. Now arrange the auxiliary condensers in the manner required; there should then be no decrease in the working aperture as seen by looking at the back lens of the objective. Whatever auxiliary lens is used, it must also be seen that the back lens of the sub-stage condenser is, at least for high powers, perfectly filled with light.

The proper use of these auxiliary condensers is a matter of considerable moment, and those who are not familiar with them are strongly advised to study, by the method already set out, the effect of altering their position, i.e. by observing a projected image on a white reflecting-surface. More information will be gained by some preliminary experimental work of this character than by reading pages of description. The worker should set up his various parts in the manner described and note the results, and should then make some variations in the relative positions of these parts and see exactly what happens. It is as important to be able to recognise any error that may creep in, and to be able to determine its cause, as it is to set up the apparatus with precision and in proper working adjustment. It is necessary to see that the iris-diaphragm, or any stop used as its substitute forming a part of the auxiliary condensing system, does not affect the working aperture of the sub-stage condenser or the objective, assuming, that is, that the beam is sufficiently large to fill the sub-stage condenser with light. The iris-diaphragm should only control the effective size of the image of the illuminant, and of the field of view. In all cases where this image of the diaphragm is the critical image focussed in the field of view, it is advisable to close it to such an extent 
that its image just encroaches on the field of view. By this means any extraneous stray light or reflections are considerably reduced, and diffraction images from portions of the object not in the field of view do not have a disturbing effect. By attending to this point carefully the purity of the resulting image is appreciably increased.

In the cases already described where one collecting-lens is placed near the source of light, and is allowed to act as a collimator, throwing a parallel beam on to a second condensinglens, it can be observed whether a parallel beam is being projected in the right way by placing a piece of white paper in the path of the rays. The illuminated area should then be quite even, with no darkened areas or bright spots in any part of it. Practically, of course, it is impossible to obtain a perfectly parallel beam of light, but a sufficient approximation to this may easily be secured.

To summarise, when using a single collecting-lens, of whatever type, the image of the lens-aperture must be projected in the plane of the object, or alternatively the aperture of a diaphragm placed close to the lens may be so focussed. With a compound system of collecting-lenses a diaphragm may be placed in a position where a parallel beam is being projected and an image of the diaphragm-aperture thrown on to the object by the sub-stage condenser. In either case steps must be taken to ascertain that the full working aperture of the sub-stage condenser is being utilised.

Any collecting-lens must be placed exactly in line with the optical axis of the microscope and with its face at right angles to the optical axis. The latter adjustment is best secured by observing the faint image that is reflected from each lens surface. The reflected image should return in the same direction as the direct beam, and can usually be seen as a more or less central dise of light on any diaphragm in the path of the rays. 


\section{CHAPTER VIII}

\section{GENERAL PRELIMINARY PREPARATIONS}

Vibration and its Prevention.-Apart from the acquisition of suitable apparatus, due consideration should be given to other points affecting the final results. Possibly the chief among these is the situation of the room or apartment in which the work is to be carried out. It has already been mentioned how greatly vibration of the apparatus is to be feared, especially when taking photographs with high powers or of a highly critical nature. It is obvious that such work is much influenced by the situation of the apartment in which the apparatus is fixed. Assuming that the work is being performed in an ordinary house, the best position for the room is either in the basement, or preferably a sub-basement or underground cellar if such exists. This should have, if possible, either a concrete or similar substantially built floor. At the Lister Institute of Preventive Medicine, to carry the instrument designed by the writer, which has already been described, two brick columns were carried down for about fifteen feet below the floor-level of the basement, and these again rested on a solid bed of concrete. While it is not contended that such is by any means essential, yet it will give an idea of how carefully such points must be attended to, when a building or portion of a building is being designed and erected particularly for this work. An additional advantage of having a basement apartment is that it is usually far less subject to rapid changes and fluctuations of temperature than is a room situated farther up or at the top of the house. In the latter the change of temperature even between night and day at any time of the year is considerable, but in the former situation it will be found that the range is small even between 
the greatest heat of summer and the low temperatures of winter. Certainly a room with a northern aspect would be better suited than any other.

It is likely, however, that the majority of workers will to a large extent have this point determined for them, and may have to devote a room to the work which is not required for other purposes. It therefore becomes necessary to point out such methods as are available for overcoming the troubles due to vibrations, such as more particularly affect the upper part of a house. It is by no means certain that any apparatus is better for being very firmly fastened down to the floor, unless that floor is of particularly solid construction, or as in the writer's case, where in the course of building the house a steel girder was placed in the floor in the position which the apparatus was to occupy. Obviously this is a refinement that can rarely under ordinary circumstances be obtained; and, where an ordinary floor is the only means of support, then some method of reducing the transmission of vibrations may be necessary.

The simplest and most easily applied is to place under the feet of the apparatus three- or four-inch cubes of solid india-rubber. This reduces the smaller tremors, and effectually prevents any but a definite shock from being transmitted. A more drastic although somewhat more troublesome method, is to sling the whole arrangement on stout cords. The illustration (Fig. 61) shows a method of carrying a small apparatus on this principle as suggested by Mr. J. I. Pigg. In this case the camera and microscope are supported on one board, which is slung between two metal uprights, themselves standing on an ordinary table. This arrangement is very efficient indeed where work has to be done in an ordinary room, and it will need to be fairly severe vibration that will affect the resulting photographs.

If the camera is of a larger type, it will hardly be possible to so hang it. In most cases where the weight is considerable it is better, if possible, to let the cords be hung from beams in the ceiling; in this case much better isolation from movements of the floor, due to persons moving about in neighbouring apartments, is obtained.

In extreme cases thick india-rubber cords may be substituted for the ordinary cord. 
Another method which would appear to be even more effective, but is more difficult to carry out, is to carry or hang the apparatus on strong steel springs. In this case it would not merely be necessary to let the weight of the whole appliance hang on the springs, but other springs should also be fixed so that they act in opposite directions to prevent the whole apparatus acquiring a periodic oscillation or vibration of its own. It will therefore be essential to connect it by several springs to one support, so that any movement imparted to it is as far as possible stopped at once whatever the direction it

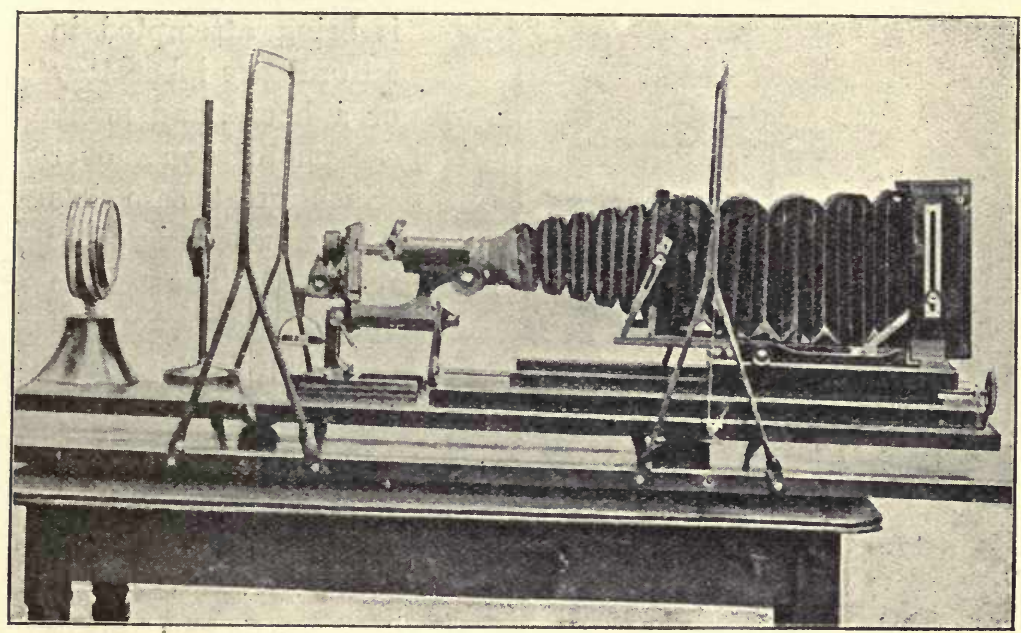

FIG. 61.-Method of supporting Camera to minimise Vibration.

may take. One method of effecting this is to have a baseboard carrying the whole apparatus on two supporting-legs at each end, both of which are cross-braced to the underside of the baseboard. A strong wooden supporting-frame is then so made that from three separate points at each end, and at each side of the apparatus, springs may be carried to the bottom of the supporting-legs. These springs require to be strong steel ones, such as may be purchased ready made for a few pence each from any good ironmongery store. It is evidently only possible to indicate in general terms how this arrangement can be carried out, as it must be to a large extent determined by the apparatus and the condition under which it has to be set up. 
But it will be found that, if properly made with due regard to the mechanical principles involved, some such spring support is perfectly efficient.

A further method, which the writer has not seen in operation but which he understands has been tried, is to float the whole apparatus in a tank of water. This obviously presents many difficulties, and is only to be regarded as a suggestion of a possible experimental method worth trying when everything else has failed.

It is not easy to overrate the effect of vibration when work of a critical nature

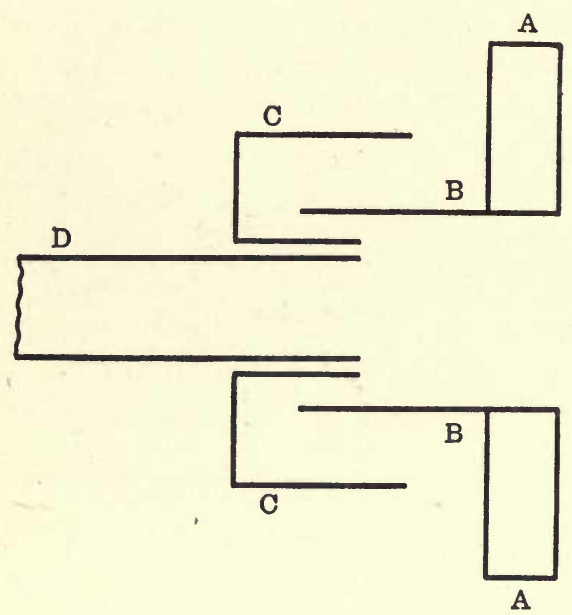

FIa. 62.-Appliance for excluding Light. is being attempted in an ordinary laboratory or a house, and many a photograph over which great care has been expended in other respects, has been spoilt by the want of attention to the elimination of this very troublesome factor.

Light - excluders and Microscope.-The microscope and camera are for all essential purposes two separate pieces of apparatus, but a connection must be made between the eye-piece end of the microscope and the front of the camera by some arrangement that will effectually prevent the entrance of extraneous light. This may be simply effected by having a velvet or cloth tube with an elastic band at either end which slips over the microscope tube and over a short tube protruding from the front of the camera. While this is quite efficient, it has the disadvantage that in the actual connecting up of the microscope to the camera there is considerable danger of the adjustments of the instrument being altered. The almost universal method is now to have a light-excluding device, as Fig. 62. This has the advantage of great simplicity in use, 
is cheap and easy to construct, and works well under all conditions. Further, there is no actual contact between the microscope tube and the front of the camera itself. Let $\mathrm{AA}$ represent the camera front, and $\mathrm{BB}$ an open tube extending from its centre. This tube $\mathrm{BB}$ may be of such a size that it can be conveniently used for putting on a carrier for a microscope objective of low power, or an ordinary photographic lens for the photographing of culture-tubes or similar objects. On the ocular end of the microscope body-tube a double tube $\mathrm{CC}$ is placed ; the inner and smaller tube is of such a size that it just slides over the body-tube, while the outer tube is sufficiently large to have at least one-eighth of an inch clearance outside the tube $\mathrm{BB}$ of the camera. When the camera front is therefore pushed up so that its tube lies between the double tube of the fitting CC, no extraneous light can enter between the microscope and camera. The whole of this appliance should be dead black to prevent the possibility of reflections from any of the exposed surfaces.

Diffused Light. - It is advisable to protect as far as possible the whole of the apparatus from diffused light, whether from the illuminant or from any extraneous source. The illuminant should therefore be enclosed in a suitable casing with an opening, other than any necessary for the purpose of ventilation, only in the direction of the optical system. Some workers also advise that the whole of the optical system should be covered with a black-cloth hood to prevent the possibility of stray or diffused light entering the apparatus. While this may be an excellent arrangement in cases where the work is carried on in a well-lighted room, yet in any special apartment where other bright sources of light are not in use, it would not appear to be necessary.

It is highly important to see that there are no disturbing internal reflections in any part of the apparatus. In well-made microscopes this is not likely to be a source of trouble, but inside the camera itself it is by no means unusual to find that there are some surfaces that are diffusing or reflecting a considerable amount of light. If this arises from the apparatus not being dead black inside, it should be immediately rectified. This can be done by taking some dilute shellac varnish and adding to it sufficient fine lamp-black to form a mixture of a creamy 
consistency. This should be rubbed over any part of the woodwork, and will effectually blacken any reflecting parts. In the case of the inside of the bellows of the camera not being dead black, it is best to make a mixture of lamp-black and ordinary flour paste, and apply this where required.

It will be found that it is a good plan to interpose deadblack metal diaphragms with suitable openings between the camera front and the ground-glass focussing-screen. Many makers supply these thin metal plates with openings of various sizes which fit into the back of the camera, immediately in front of the position taken by the dark-slide. It is advisable to use one with as small an opening as possible when photographing. Only harm is done by allowing useless portions of the image to fall on the photographic plate, since they are then diffusing unnecessary light inside the camera body. If the camera bellows are in segments, a metal diaphragm may be usefully interposed about midway between the camera front and the photographic plate.

Heat from Illuminant. - With most high-power illuminants some method of absorbing the radiant heat is necessary. This is particularly the case with the electric arc and lime-light, and when either of these is in use it is advisable to ensure that no unnecessary heat reaches the microscope. Otherwise expansion of the components is sure to take place, and sufficient dislocation will ensue to alter the focus during an exposure of any considerable length, with the result that the image is not well defined. This may be prevented by placing a water-bath in as near a position as convenient to the source of light. Such an appliance is sold by most opticians ready made, but an improvised one may be constructed in the manner described for making cells to contain coloured fluids, referred to in a later chapter on this subject. The cell should be filled with wellboiled distilled water, to ensure that all the air is driven off; otherwise the heat of the radiant will be sufficient to cause airbubbles to appear on the walls of the containing-vessel. It is sometimes advised that the absorbing fluid should be a saturated solution of alum instead of water only, but this would not appear to increase the heat-absorbing power very greatly. In cases where a yellow screen is used, then a weak solution of perchloride of iron may be substituted for plain water, and will 
much more effectively absorb the heat rays. The greater the heat emitted by the radiant, the larger the water-bath should be, so that there is sufficient water to prevent any considerable rise of temperature and consequent injury to the objectives during a long exposure. In any case the cell should be at least one inch in thickness.

Diaphragms. - It is convenient to interpose between the source of light and the microscope one, and in some cases two, diaphragms, either of the iris pattern or of the type in which a series of metal plates with openings of various sizes can be inserted at will. It is usual to have one of these as close to the illuminant as possible. In the case of illuminants of rather large area-such as the mercury-vapour lamp-one may be placed almost in contact with the tube of the lamp itself. It is well that any part of the illuminant that is not required in actual use should be effectually screened off. In cases where a system of collecting-lenses-such as the Zeiss-Kohler lenses already described-are being used, then a diaphragm may also be interposed between the two lenses. It is, with any system of illumination, advisable for the illuminating beam to be contracted to such a size that no portion of the beam, other than that actually in use, is allowed to reach the microscope. In any case where one or two diaphragms are used the position should be chosen so that any extraneous or unused light is effectually screened off. The iris-pattern diaphragms are obviously the most convenient, but the cheaper metal plates with suitable sized openings are perfectly efficient.

Size of Camera.-This is a point actually of no particular importance, but where a camera is being obtained especially for the purpose in view, it is advisable that its size should not be greater than is really necessary. In the large majority of cases what is known as a quarter-plate camera is sufficient, as in any other than low-power work the area of good definition will rarely exceed that of a circle of three inches ; consequently, the plate of $3 \frac{1}{4}{ }^{\prime \prime} \times 4 \frac{11}{4}$ (quarter-plate) will be sufficient for this purpose.

When using Planar or similar low-power lenses, however, the area of good definition is considerably larger, so that it may in general be more convenient to adopt a half-plate size, $6 \frac{1}{2}{ }^{\prime \prime} \times 4_{4}^{3 \prime \prime}$. This will entail the use of plate-carriers when using the plates of 
small size for high-power work. It is important to see that these plate-carriers do not interfere with the register of the plate in any way ; that is, that a smaller or larger plate when put into the dark-slide should both be in exact register with the focussingscreen of the camera. In general, these conditions are exactly carried out by camera-makers, but it is advisable to make sure that there is no difference in their position before work is actually attempted.

This may be done roughly by measuring with a rule the distance of the focussing-screen, or of the sensitive surface of the photographic plate, from any fixed position in the camera itself. This is not such an efficient method as an optical one. An ordinary photographic lens of wide aperture should be put on to the camera front and some sharply defined object-such as a window frame, or even better a sheet of printed matter-carefully focussed on the ground-glass focussing-screen. The camera back should then be inserted, and, assuming that it is a double back which will allow of one-half of the slide being turned back and the plate in position being exposed to view, a piece of groundglass may be put into the dark-slide in the position usually occupied by the sensitive plate. The image is then observed, and should, under these conditions, be equally well defined when viewed on either the proper focussing-screen or on the ground-glass inserted in the dark-slide carrier.

It must be admitted, however, that in photo-micrography such perfect registration under general conditions does not affect the final result to the same extent as in ordinary photography. The angle of the beam is usually so narrow-that is, the rays approximate so closely to parallel rays-that a small difference in position between the dark-slide and the focussingscreen does not have so great an effect. Since, however, the camera may be used for varying classes of work, it is a point well worth attending to at the start; so that whatever may be attempted no depreciation of the result need be anticipated from such a source of error.

If greater exactness is required, a piece of plain glass may be substituted for the ground-glass, and the image focussed by a good focussing-magnifier. This method is both easier and more efficient than taking a succession of photographs to ascertain the correctness of register of the dark-slide or its carriers, as any 
possible aberration, due to the lens with which the test is made, is then entirely eliminated.

One point of considerable importance, affecting both the dark-slide and the focussing-screen, is that they should drop into their position at the back of the camera easily without jar or dislocation of any part of the apparatus. The method of inserting either the one or the other in a long groove should therefore be avoided, and it is sufficient that they drop in at the back either in a very short groove, or even better, are merely held in position with a simple form of catch. It is also important to see that the shutter of the dark-slide moves very easily and sweetly when it is being withdrawn; nothing is more disconcerting than for any unforeseen jar to disturb any part of the apparatus as the result of removing the focussing-screens, inserting the dark-slide, or withdrawing the shutter, and too much attention can hardly be given to these points to ensure that all the movements are perfectly smooth and can be made with the least possible risk of disturbance. As the room in which the work is being carried out should be either partially or completely darkened, there is very little risk of extraneous light entering the camera at this point; so that it is not nearly so essential to ensure that the junction between camera and dark-slide is absolutely light-tight, as in the case of a camera used in the field.

Focussing-screens.-The camera should be provided with two focussing-screens : one of ground-glass and the other of plain glass - the latter to be patent plate to ensure that there is no inequality of surface. The ground-glass screen should be finely but well ground. It is not recommended that the ground-glass screen or its substitute should be extremely fine, for in the writer's experience this is a point that may be carried too far. If a screen is too translucent, then such a large amount of light is lost by direct transmission that the image itself loses in brilliancy. On the other hand, with too coarse a screen the grain of the screen itself becomes so painfully obvious when focussing that the fine details of the image are lost. It must, however, be pointed out that a great deal of the focussing of ordinary photo-micrographic work is best carried out on the ground-glass screen, so that careful attention to this point is a dvisable. 
There are many substitutes for ground-glass that have been recommended at various times, but they nearly all have the disadvantage that although their grain is fine, the screen itself is too translucent, thus resulting in considerable loss of light. In practice it will be found that ground-glass that is a little coarser than the finest that can be obtained is the most efficient, and, if used in conjunction with suitable focussingglasses to be described, there are many cases where exact focussing can be effected without recourse to any other screen. In cases where fine detail and a particular portion only of an object is to be focussed, then recourse must be had to the plain-glass screen. This should have some scratches made by a diamond in the direction of the diagonals of the plate, and these scratches should be carefully focussed with the focussing eye-piece before any actual focussing of the microscopic image is attempted.

An alternative method, and one which may be adopted where only the ground-glass screen is provided with the camera, is to cement on to the centre of the screen with Canada balsam an ordinary microscopic cover-glass. The Canada balsam has the effect of making this small area perfectly clear, effectually eliminating the ground-surface, and the image can therefore at the same time be observed over the whole area of the screen. For fine focussing purposes this central clear area will often be found sufficient, but it is open to the objection that the clear area is very limited, and consequently, should the part to be focussed lie at any other spot than the centre, it cannot satisfactorily be observed.

A substitute for the ground-glass surface may be made by coating a piece of patent or thin plate-glass with a matt varnish made as follows :-

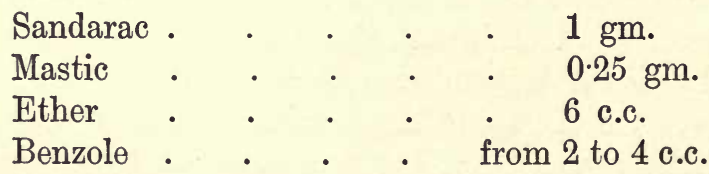

The finer the surface required, the greater the degree of dilution of this solution-that is, more benzole should be added so that a finer film is deposited on the glass. This will give a very fine focussing-screen, but it is open to the objection that the matt surface is very liable to abrasion, and in course of work, 
where a screen is handled a good deal, this becomes a serious disadvantage.

The finest focussing-screens are those made by chemical means. Take an ordinary photographic plate, preferably an extra-rapid one, and without any preliminary exposure to light, immerse it in the dark in any non-staining developer free from potassic or ammonic bromide. The plate may be left in this for from five to twenty minutes. The longer periods result -in a fine and translucent screen, and the shorter periods in a screen that is equally fine and free from granularity, but less translucent. At the end of this period of development, the plate has to be washed and fixed in the ordinary way. It should then be immersed in a solution of iodine in potassic iodide; the exact strength of this solution is not important. It may be left in for some few minutes until the plate is completely iodised, then again washed and immersed in dilute ammonia, and finally washed and put to dry. This screen has, in the hands of the writer, proved extremely useful when work of a critical nature is being carried out and when the plain-glass screen does not fulfil its purpose. If used in conjunction with a focussing-ocular, the grain is so fine that there is no evidence of it, even if the ocular is a fairly high-power one. The difficulty of accommodation that occurs in some cases when using a plain-glass screen, is entirely absent when some such fine-grain screens, giving a concrete image, are used.

The ground-glass screen may be rubbed over with a small quantity of oil in cases where it is too coarse, or where a feeble source of light is used and it is not sufficiently translucent. It need hardly be mentioned that the ground-surface of the groundglass screen, or in the case of the plain screen, the surface which has the diamond scratches on it, should be towards the light.

An extremely useful accessory is a white opaque screen, placed in a suitable position so that the microscopic image can be projected on to it, and this image on the screen observed to see if the centration or other adjustments of the illuminant or optical system are correct. With an apparatus of the type in which the microscope and accessories swing out of the optical centre, the image can easily be projected on to such a white screen if placed in a position at either side of the camera back. 
If reference is made to Fig. 47 one method of arranging such an opaque screen is seen. It consists essentially of a piece of plain board which is hinged on to the side of the frame carrying the focussing-screen of the camera, and on the side towards the microscope is fastened a piece of plain white paper or cardboard. The white surface is in alignment with the focussing-screen of the camera, but as it is hinged the position may be altered when the microscope is swung out. Obviously this screen must be at a different inclination to the optical axis according to the length of camera in use, so that the hinges allow of variation of the inclination. The image may be approximately projected on to the opaque screen in the position in

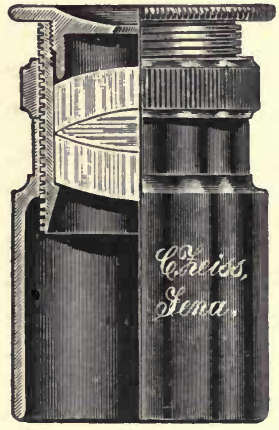

FIG. 63.-Focussingglass. (Zeiss.) relation to the microscope that it would occupy on the ground-glass focussing-screen. With this arrangement it is possible to make all the adjustments - centreing of the sub-stage of the microscope, centreing of the object, focussing of the projectionocular diaphragm, approximate focussing of the objectitself-and to observe finally if all the adjustments are correct, while standing in a position near the microscope. It is obvious that this renders the work of adjustment very much easier, and any correction can be made without having to move backwards and forwards from the ground-glass screen to the microscope, while the effect of any small alteration that may be desirable is immediately seen. On this, as well as on the ground-glass screen in the camera, diagonal lines should be drawn so that their point of intersection indicates the position that should be occupied by the centre of the object. It is also useful to make a circular mark that indicates the largest image that may be photographed on the sensitive plate to be used. In the case of a quarter-plate this circle could not be more than three inches in diameter.

Focussing Eye-pieces.-When using the plain-glass focussing-screen an achromatic eye-piece, usually of the Ramsden type, must be employed. There are many types of these on the market, and the one illustrated (Fig. 63) is typical. It must 
be so adjusted that when placed on the plain focussing-screen the diamond lines on the far side of the screen are exactly in focus. This is an adjustment that must be made for each individual user; but, having once been carried out, it remains always in the correct position ready for use. The adjustment is effected by placing the eye-piece on the focussing-screen until the image of the diamond line or of the ground-glass surface is sharply focussed; this is done by screwing the cell carrying the optical portion in or out by means of the milled head shown. When this operation has been carried out, any alteration of the position of the lens system is prevented by firmly screwing down a clamping-ring, which prevents any alteration during use.

In general, these focussing eye-pieces are made of too high power for photo-micrographic work, as the image-at least in high-power work-is not one that will stand any great amplification, especially when the focussing-glass is not well achromatised. The writer has had made by Messrs. J. Swift \& Son a focusser which he has found answer better than any of those on the market. It is a well-achromatised system, but of very low power, so that the image is magnified less than two diameters. In effect it only takes up the image at the focal plane, and transmits it to the eye with but slight alteration. The only objection to it is that the field is small, but in the large majority of cases this is of no moment.

In actual practice such a focussing system is fitted into a long mount, and owing to this it can only be used at or near the centre of the focussing-screen. If it is away from the centre to any considerable extent, the image is not transmitted, but is cut off by the sides of the mount. The great advantage of having such a low-power eye-piece for focussing is that the image is transmitted practically unaltered, and it is much easier therefore to focus images formed by high powers.

Some workers have advised the use of ordinary spectacle lenses for focussing, and this would appear to be quite practicable where a ground-glass screen only is used. Better still is it to use a pair of spectacle lenses mounted to form a binocular focussing-glass. This type is made in a convenient form by Messrs. Newman \& Guardia, and is supplied by them for use in their reflex cameras. The binocular, as supplied, should be 
mounted on a simple tube or box, or in some similar way, so that the distance of the eye-pieces from the focussing-screen is equal to the distance at which they are placed in the reflex camera for which they are originally intended.

Position of Microscope in Relation to Camera.-A point of primary importance, although it is not often referred to, is to ensure that the optical system is in perfect alignment, both vertically and horizontally, with the camera. This is not always the case, even though the apparatus is made especially for the purpose. If the microscope and other optical parts swing out of centre, as already described, it may happen that they do not return exactly to a position in which the optical axis is directly in alignment with the centre of the camera; or, again, the microscope itself when brought into a supposed horizontal position may not be truly horizontal. Any want of truth in either of these directions may be determined with exactness by observing an image on the focussing-screen of the camera. As a test-object for this purpose nothing is better than an ordinary glass microscopic slip, on which two fine lines have been ruled with a diamond at the centre of the slip exactly at right angles to one another. These are focussed in the microscope with a medium-power objective, and the image is projected on to the ground-glass screen so that the point of intersection of the diamond lines is truly in the centre of the screen of the camera. On moving the back of the camera, carrying the focussing-screen, backwards and forwards from its greatest to its shortest length, no alteration whatever in the position of the image should be observed. This condition is in practice very rarely exactly complied with, and more often than not it will be found that some alteration, either laterally or vertically, occurs in the position of the image. Careful observation will at once show in which direction there is any deviation from correct alignment of the apparatus, and steps must be taken to rectify this. It is evident that the direction of the movement of the object on the screen will be in the opposite direction to the direction of error of alignment. Any such error will often be traced to the microscope itself, since in placing it in position on the baseboard, reliance is usually placed on the tripod foot itself being exactly true. Vertical deviation may also generally be traced 
to the same source, as in even the best microscopes it is not unusual to find that the optical axis of the instrument is not truly horizontal. These errors are most easily detected by watching the projected image in the manner described, since this forms a far more exact test than any method of direct measurement.

Methods of Exposing. - In many ready-made cameras an exposing-shutter is fixed at the front, which is actuated by mechanical or other means. The great disadvantage of all such arrangements is that there is the danger of some vibration being imparted to the apparatus; and as such vibration obviously occurs at the moment when an exposure is being started, the result may be most harmful.

It will be found far more satisfactory to have some very simple arrangement for effecting the exposure. The simplest of all is merely to interpose between the microscope and the nearest auxiliary condensing-lens a piece of blackened cardboard. This may be supported in position as near to the microscope as possible, but yet not touching any part of the instrument, and may be removed at the commencement of the exposure and replaced in its position at the end. It will not be found, at least when working in a room which is even partially darkened, that a sufficient amount of reflected light enters the camera to have any effect on the photographic plate. In cases where the work is carried on in a well-lighted room, some arrangement inside the camera may become necessary. The simplest way is then to have a thin metal plate, which should be as light as possible, carried inside the camera front on a hinge or thin spindle, with freedom to rotate. The plate should be rigidly connected to the spindle, and the latter may project through the front of the camera on either side, with a milled head outside the camera front which may be actuated at either or both ends. On rotating the milled head the plate should be so arranged that it either completely closes the aperture in the camera front, or moves downwards and lies in the bottom of the camera, leaving therefore a clear aperture where the microscope and camera join.

Alternatively any of the mechanical photographic shutters may be used, and may be fixed in the front of the camera. But unless one of the modern between-lens shutters of good 
construction is fitted-and this is really an unnecessary and expensive luxury-it will be found that some vibration is bound to take place.

It should be mentioned that when using very low powers the blackened card or its substitute may be interposed between the microscope objective and the object, thereby minimising to a considerable extent the chance of reflected light entering the apparatus. It is obvious that such reflections are more likely to occur with low-power lenses than with high-power ones, since the former transmit so much more light.

Various methods for effecting the exposure will suggest themselves to the worker, and any one may be adopted that does not result in disturbance of any part of the apparatus, and yet ensures that the sensitive plate is protected against stray light.

Focussing.-The various appliances for properly focussing the image have been already described, but there are one or two further points that may arise in the course of their use. It is of course necessary that any worker should first become conversant with the use of the fine-adjustment on the microscope under the ordinary conditions of visual work, and it is assumed that this has been already mastered.

In photo-micrographic work the fine-adjustment is usually actuated by means of some long focussing-rod, which the observer can rotate at one end, and which is connected at the other end to the fine-adjustment milled head on the microscope itself. The various types of these appliances have already been fully detailed. In actual practice, whichever focussing arrangement is adopted, it should act on the fine-adjustment with precision. It will be seen in general that one of the arrangements, in which thin cords are taken over the fineadjusting head very lightly, are in practice preferable. The arrangement should be such that the image only moves in and out of focus when the focussing-rod is rotated, and so that no lateral displacement of the image occurs. The rigid method of focussing adopted in the Zeiss model, in which the motion is imparted to the fine-adjustment by means of a Hooke's joint, usually causes some slight lateral movement of the image when used with high powers, although it must be admitted that the actual focussing can be done with great exactitude. 
The movement of the image is perhaps only what might be anticipated; for the weight of the moving parts is such that there is considerable thrust, and it is only natural that this should become evident when the mechanism is actuated and the direction of movement reversed.

In the greater part of high-power work it will be found that the plain-glass screen, used in conjunction with a focussing eye-piece, will be the most satisfactory ; but it often happens in bacteriological or similar preparations that it is easier to do the fine focussing on the ground-glass screen. It must be remembered that these minute bodies are themselves either cylindrical or spherical, so that there is no absolutely sharp edge on which to focus. It therefore often follows that a better idea of the actual image is conveyed when it is seen on a screen that gives a concrete image.

It may happen with high-power illuminants that the image is too brilliant to focus with safety on the plain-glass screen by means of a focussing-ocular. In this case on no account must the sub-stage condenser-diaphragm be contracted; apart from the fact that the optical conditions are being interfered with, there is always the risk of some slight de-centration of the sub-stage condenser, if the diaphragm is interfered with at such a late stage of the operation. If there is too much glare, then the light must be modified by placing some bluish-grey glass, or a piece of the colour supplied by some opticians for this purpose-a dark blue-greenbetween the microscope and the source of light. An effective glass is to be obtained from Messrs. Hetley of Soho Square, which entirely conforms to the required conditions, as it appears simply to reduce the quantity of light transmitted without in any way altering its colour-value. This is a matter of considerable importance when colour-screens are in use; for, should a glass which has a power of selective absorption be used, it would disturb the arrangements already made and affect the resulting image. It is, of course, understood that any light-modifier of this description is removed before the actual exposure takes place.

It is perhaps almost unnecessary to point out that the focussing should as far as possible be carried out at the centre of the field-that is, with the centre of the field in the 
microscope projected exactly on the centre of the ground-glass focussing-screen. It may happen that the particular point in an object that is required to be shown up, even at the expense of other parts, does not lie exactly at the centre of the field, and in this case of course the above rule will have to be departed from. But in general it will be found more satisfactory to focus one central point in the object and allow the remainder to take its chance. This applies particularly when using apochromatic objectives, since unfortunately their field is small; it therefore becomes more important that the essential part of the image should be central rather than to attempt to focus any zone that lies away from the centre.

The apparatus described on p. 119, as devised by Mr. J. W. Gordon, provides that when the object is visually in focus, the camera has only to be placed in position and the photograph taken, when a perfectly defined photograph results ; in other words, no additional focussing is required between the observation of the image and the actual process of obtaining the photograph. While this is unquestionably extremely convenient where only records of researches are desired, and not highly critical photo-micrographs, it is, however, a method that does not lend itself to very general use. In Mr. Gordon's arrangement the photographs are quite small; in fact, the sensitive plate occupies a position very close to the top of the microscope tube, so that magnification is obtained entirely by combination of objective and ocular.

The application of this method to general photo-micrographic work was described as long ago as 1902.1 It is effected by substituting for the ground-glass screen a minus spherical lens, and using this for focussing purposes. Such lenses may be obtained from an optician, and they are usually supplied in a series from $-1 \mathrm{D}$ to $-12 \mathrm{D}$. The lens that should be used for a particular magnification depends upon the eyesight of the operator, and the selection of the particular lens for a given magnification must be determined by taking a photograph. Instead of focussing on the ground-glass screen, the spherical lens is placed over the ocular, and the image is focussed through this lens. The latter is removed before exposure-the

1 Journal of Applied Microscopy, pp. 2082-84. 
photograph therefore not being taken through the lens itself. The use of the lens is simply an arrangement to enable the eye to see the plane of the preparation that is projected on the ground-glass. The authors of this method recommend that trial photographs should be taken with an object such as a stage micrometer, and that this should be photographed with different combinations of lenses and length of camera, so that the lens to use for a given magnification may be determined.

Obviously this method has its limits, since it only enables the worker to obtain certain magnifications for which he has a focussing-lens. Further, the accommodation of the eye, which in some cases is very considerable, is not allowed for; hence it often happens that an object which seemed visually to be in focus, is only so in consequence of some strain on the eye, and when the image is recorded on the photographic plate the result is by no means sharp. It would appear, therefore, that for general work, where widely different magnifications are required and preparations of varying character have to be photographed, the only practicable method is that originally described, viz. to focus the image in the plane that will ultimately be occupied by the photographic plate.

Magnification.-The magnification of microscopic objects is usually expressed in diameters, and not in terms describing superficial area. An object is therefore said to be magnified a certain number of 'times' - this referring simply to the number of diameters that it is enlarged. The same general conditions apply as to any enlarged image projected by an ordinary lens-such as a photographic or a projection one. The magnification therefore of a projected image if the superficial area is considered varies to exactly the same degree as the intensity of illumination, i.e. as the square of the distance. In visual work magnification is estimated at a distance of ten inches from the eye-lens of the eye-piece, as this is the proper distance for normal vision. As a rule, achromatic objectives are described as of somewhat longer focus than they actually are, so that a one-inch objective is often something less than that, and may be as short as threequarters of an inch. An objective of exactly one inch focal length would magnify ten times at a distance of ten inches 
from its upper principal focal plane. It is sufficiently accurate for all ordinary purposes to measure this distance from the upper lens of the objective, so that an image projected into the camera at a distance of ten inches from that point would give a magnification of ten times. If the camera-length is increased to double the above dimension, the magnification expressed in diameters is then doubled, and therefore becomes twenty times, and so on, for any increase of distance. We therefore get for this distance of ten inches the following as the initial magnifications to be obtained with lenses of the indicated focal lengths :-

$$
\begin{aligned}
& \text { A lens of } 1 \text { inch focus or } 24 \mathrm{~mm} .=\times 10
\end{aligned}
$$

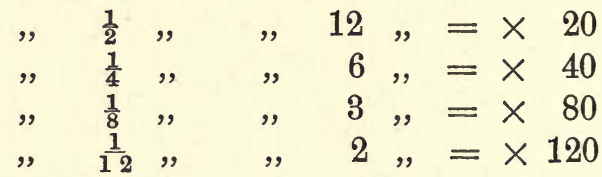

These are all on the assumption that the focal length of the lens is exactly that indicated; but it rarely happens that on actual test this is found to be the case.

In photo-micrographic work there are three factors that can influence magnification: the power of the objective, the power of the ocular, and the length of the camera. It is therefore possible to obtain a certain magnification with different objectives-that is, within limits-and the method that may be adopted to obtain any given magnification must always be determined with reference to the object itself.

It has already been pointed out that the quality of penetration, for instance, depends on the N.A. of the objective, and in general this N.A. is dependent on the focal length of the lens. In cases, therefore, where an object is of considerable thickness, and where it is necessary to obtain the best compromise possible of varying planes in it, it would be advisable to use a lens of the greatest focal length, and to obtain the necessary magnification either by using a higher power ocular, or by increasing the camera-length. No definite rules can be laid down in this matter, and it is only by careful consideration in relation to the object itself and after perhaps a fair amount of experience, that the best combination can be exactly determined. 
Apochromatic objectives are in general much more accurately described than achromatic ones, so that the margin of error in estimating magnification solely in reference to the indicated focal length of the objective is likely to be quite small. Their focal length is described in millimetres, and the chief ones are indicated in the foregoing table. The magnifications set out are their initial ones, and on placing an eye-piece on the microscope an image projected at ten inches' distance from it will be as many times larger as the initial power of the eye-piece ; that is, assuming that we take a No. 2 projectionocular, the magnification at ten inches' distance from the front lens of the projection-ocular will be twice that to be obtained without the latter. When the image is projected, e.g. with apochromatic objectives and no ocular, the initial magnification indicated is obtained at a distance of $160 \mathrm{~mm}$., or in the case of long-tube objectives $250 \mathrm{~mm}$. from the objective end of the microscope body-tube. When a projection-ocular is added, the image, magnified 2, 4, or more times according to the ocular, is then obtained at a distance of $160 \mathrm{~mm}$., or 250 $\mathrm{mm}$. measured from the ocular itself and not from the objective.

With projection-oculars it is fairly safe to assume that their magnification is in accordance with the number engraved on them, so that a $\times 2$ or $\times 4$ projection-ocular will magnify the image two or four times as indicated. But should the ordinary eye-piece be used, indicated by the letters A, B, C, and so on, or by some arbitrary number, then the only way is to determine their magnification by the projection of a micrometer ruling.

The influence of camera extension is very similar in effect to a change of ocular. If the ground-glass screen is ten inches from the front lens of the ocular, we then obtain magnification in proportion to the focal length of the objective, multiplied by the number of times that the ocular magnifies. When the camera is extended beyond the ten inches, the amplification is then directly proportional to the increase of camera extension. For instance, if we place an object on the stage with a one-inch objective and, say, a No. 8 eye-piece, we should find the object to be magnified approximately 80 times at ten inches' distance. But if we double the camera-length we should now get an object twice that size, equal to a magnification of 160 times.

It is most convenient on any photo-micrographic apparatus 
to have a scale placed at the side of the camera showing the extension from the front of the ocular. If an exact scale in inches or centimetres is not adopted, a series of marks showing each ten inches of extension will be found very useful. It is then only necessary to observe the length of camera that is being employed, taking into consideration the ocular and the objective, when by a very simple arithmetical calculation the magnification that is being obtained on the ground-glass screen can be approximately estimated. For most objects such an approximation is sufficient, and it is only in cases where an exact estimate of the magnification employed has to be obtained that other methods need be resorted to.

If the estimation of magnification is not sufficiently accurate when arrived at by simply considering the focal length of the lens, the power of the ocular, and the camera extension, then recourse must be had to the projection of the image of a micrometer ruling. This is simply a $3^{\prime \prime} \times 1^{\prime \prime}$ glass slip, on which is mounted a cover-glass ruled by a diamond at fixed distances apart. The rulings are generally one-tenth and one-hundredth of a millimetre. It will be best perhaps to carry out the first estimate of magnification by means of a medium-power objective-say, one inch. Turn the microscope in the horizontal position, having got the ruling on such a micrometer in focus, and project the image on to the ground-glass screen of the camera. The screen should preferably be at a considerable distance, if the camera will admit of it-say, five feet from the front lens of the objective. If the camera extension will not admit of an image being projected to this distance, then it will be sufficient, if the source of light is fairly powerful, to throw the image on to a white screen or card at the required distance. We thus have a projected image at sixty inches' distance, and on reference to this image if we find, say, that the rulings, each of one-tenth of a millimetre apart, when projected on the ground-glass screen, have a distance between them of $6 \mathrm{~mm}$., we shall know that the initial power of the objective is ten, and that the lens therefore is correctly a one-inch objective. Similarly, if we take any other lens and divide the magnifying power obtained by six, the result again will give the initial power of the lens at a distance of ten inches. With higher powers it will be necessary to use the one-hundredth 
millimetre scale. The reason why it is advisable to take a long camera-extension is that the distance should be reckoned from the posterior principal focus of the lens, and this is not a point easy to determine. Over a long distance the error becomes so small that it is not really of great moment, and the distance may be measured from the front lens of the objective.

Another small error is introduced owing to the fact that the farther the image is from the objective, the nearer the lens has to be focussed to the object to get the projected image into correct focus, but this again is not sufficient to disturb the result for ordinary work. It must be carefully noted in making any estimation of magnification that in cases where an ocular is used it only holds good for the particular tube-length with which the determination is made. It is not advisable in determining magnification by the aid of a micrometer to rely upon the distance between any two rulings, but rather to take a number and then to take the mean of these; there is usually some error in individual rulings, and this is reduced to a minimum if the mean is taken of several. Where ordinary oculars are used the determination must be made with each objective in combination with each ocular.

As examples of magnification to be obtained with varying types and makes of objectives, the following are the results with some in the hands of the writer. Table IV is really an abbreviation of one kept at hand for reference when any given magnification is required.

TABLE IV

\begin{tabular}{|c|c|c|c|c|c|}
\hline Objective. & Ocular. & Tube-length. & $\begin{array}{c}\text { Magnification } \\
\text { with Camera } \\
\text { at 2 ft. }\end{array}$ & $\begin{array}{c}\text { Magnification } \\
\text { with Camera } \\
\text { at 7 ft. }\end{array}$ & $\begin{array}{c}\text { Increase of } \\
\text { Magnification for } \\
\text { each inch of in- } \\
\text { crease in Camera } \\
\text { Extension. }\end{array}$ \\
\hline $35 \mathrm{~mm}$. & None. & - & 15 & -62 & - \\
$1 \mathrm{inch}$. & None. & - & 26 & 100 & 1.26 \\
$16 \mathrm{~mm}$. & 2 & $160 \mathrm{~mm}$. & 44 & 230 & $3 \cdot 1$ \\
$\frac{1}{5} \mathrm{inch}$. & None. & - & 150 & 470 & 5.3 \\
$3 \mathrm{~mm}$. & 2 & $160 \mathrm{~mm}$. & 270 & 1500 & 20.5 \\
\hline
\end{tabular}


To ascertain the dimensions of a microscopic object, the photographic method is probably the most accurate. If the magnification is first exactly determined it is only necessary to carefully measure the size of the object as seen in the photograph, and then to divide the measurement so obtained by the magnification. The result gives the actual size. With regular recurring structure, such as in diatoms, it is best to measure the distance covered by a definite number of markings-for example, ten-and to divide the result again by this number. Any error that there might otherwise be in taking a measurement between the exact centre of any two lines or spaces is by this means considerably reduced.

The Dark-room : Its Illumination and Appointments.The proper equipment of the dark-room is a matter of some importance, although it will generally happen that some provision in this direction has been previously made; it will therefore be a question of utilising what already exists rather than installing an entirely new equipment.

The main questions in fitting up a dark-room are its illumination, water-supply, ventilation, and regulation of temperature.

The illumination is the chief point, as the method of effecting this will greatly influence the photographic results, and it must be largely determined by the class of work to be carried out. The usual source of light is what is known as a ruby lamp, this being some ordinary white light of low intensity - such as an oil lamp, gas, or electric light-which is enclosed in a light-tight box, the latter having in one or more sides of it a piece of ruby glass. As in photo-micrographic work plates are used which are sensitive to a considerable range of the spectrum, an ordinary ruby glass is not sufficient; in fact, most ruby glasses that are sold for photographic purposes are not by any means safe even with ordinary plates, especially if these be highly sensitive. No plate is entirely insensitive to any portion of the visible spectrum ; it only requires to be exposed to any source of light for a sufficient time for an effect to be produced. It will be advisable to have one thickness of rather deep ruby glass and to supplement this with either another piece of a deep orange colour or preferably a piece of ruby with one suriace ground. It is usually better to have some 
diffusing medium, such as the latter, rather than plain glass, as the light is thereby better distributed over the working area. The amount of light that is permissible depends entirely on the plates to be used, but in any case it is not advisable to reduce the illumination of the room to too great an extent; not only does this entail some strain on the worker, but it is also much more difficult to observe the progress of the plate during development. Anyhow, whatever plate is used, means should be taken to shield it as much as possible from the darkroom light during all the operations. For bromide-printing processes or lantern-slide making, one thickness of orange glass will be sufficient, as in neither of these processes are the emulsions so sensitive. In working with panchromatic plates, which are sensitive to practically the whole spectrum, recourse must be had to one of the new green safe lights. Nearly all photographic plates are less sensitive to a narrow band in the spectrum in the region of the green, and it has been possible, by making a dark-room light of such a colour that it only transmits green in this particular region, to work in comfort with plates that cannot be safely exposed to any red light. Such green colour-screens ean now be obtained commercially either from Messrs. Wratten \& Wainwright or from Messrs. Sanger, Shepherd \& Co., and these are in practice by far the best illuminants for the dark-room. They may be obtained in practically any size to suit the particular form of lamp in use. It is advisable to provide against their being subjected to any considerable amount of heat, so that the lamp should be one in which the illuminant is not too close to the screen. In some forms of lamp it is possible to place a glass cell in position, and to fill this cell with a solution composed of dyes which transmit such light as is required. The advantage of such a light is that there is less general absorption and the quality of the light can be exactly varied at will. One form of these is a cylindrical glass vessel in which an electric lamp is suspended, so that it is only necessary to fill the vessel with a coloured solution to obtain an efficient means of illumination. Alternatively, gelatin or collodion films may be dyed in the manner to be described for making coloured filters, but these require using with care since they are rather subject to change as the result of either overheating or prolonged exposure to light. 
To make a dark-room lamp of the tank form suitable for work with colour sensitive plates, a solution such as the following may be used. To 100 c.c. of water add one gramme of aurantia, and 0.25 gramme of methylene-blue. The exact strength of solution should be determined by means of a hand spectroscope, as the light transmitted will, of course, depend on the thickness as well as on the strength of the solution; so that the strength should be such that it transmits a band of the spectrum limited between 5600 to $5800 \lambda$.

Another dark-room safe light, suitable for a lamp of the liquid-cell type, may be made up with a solution as follows :-

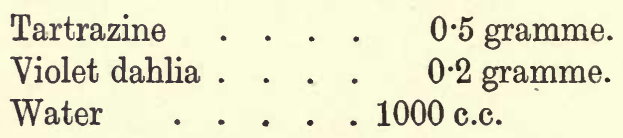

The solution should be filtered. This screen is suitable for all plates that are sensitive to yellow and green, and would therefore be suitable for most of those of the orthochromatic or isochromatic type. For very rapid or panchromatic plates, the quantities of the above dyes should be increased, and may in extreme cases be even doubled. It is stated that with a ten candle-power incandescent electric lamp used behind such a screen no fog is caused on panchromatic plates even when development is prolonged. The thickness of the cell should be approximately one inch, but may be less than that where an illuminant of lower intensity is used.

The ordinary photographic worker who is accustomed to red light in the dark-room at first gets the impression that green light cannot possibly be safe for his plates; but this is entirely owing to the fact that the eye is much more sensitive to green than it is to red, so that a smaller quantity of green light can be safely used than is possible when using red light. After having once become thoroughly used to this method, it will be found that it is easier for all classes of work, and probably less harmfal to the eyes of the worker, than any other source of light.

The dark-room illumination should never be effected by daylight, as the source of light is too variable, and, further, the effect of daylight on either ruby or orange glass or on the green glass filters, is very marked. In any case, every few months the dark-room light should be tested to ensure that no alteration 
has taken place in the quality of the illumination. This may best be done by putting a plate into an ordinary dark-slide, withdrawing the shutter half-way, and exposing the plate to the dark-room light for a minute or two; on development, no difference should be noticed between the part of the plate which has been exposed to the light and that shaded by the dark-slide shutter.

It is desirable that the dark-room should be kept at as regular a temperature as possible. The variation between summer and winter is so great that unless some means of regulating the temperature is adopted the time taken to develop plates will vary within very considerable limits ; further, the sensitiveness of the plates themselves would appear to be affected to a marked degree, so that one and the same plate would require somewhat more exposure during winter than during summer. The situation of the dark-room, therefore, should if possible be such that it is not subjected to great differences of temperature, and the necessary conditions can best be obtained if the room is in the basement of a house, and has a northern aspect.

A good water-supply is essential, and the tap should give an easy flow, and be provided with a rose to give a fine spray of water if so desired.

It is convenient to have an arrangement for rocking the dish during the process of development, and if a slow developer is being used, it is then quite practicable to place the plate in the developer, cover the dish so that it is protected from light from the dark-room lamp, and start the rocker, leaving it for a few minutes for development to commence. There are many rockers, simple and otherwise, on the market; but a perfectly efficient one may be improvised by anyone with but a small knowledge of carpentry.

Take a piece of any hard wood about $8 \mathrm{in}$. by $6 \mathrm{in}$. by $1 \mathrm{in}$. and on one of its longer edges screw firmly in a direction at right angles to its surface a strip $3 \mathrm{in}$. by $1 \mathrm{in}$., and about $3 \mathrm{ft}$. long. At the middle-line of the first-mentioned piece of wood which supports the dark-room dish, put in two round-headed screws, one on either side, so that if the arrangement is now placed on the dark-room bench it is free to rock on the heads of these screws, allowing the long strip of wood to hang over 
the side of the bench. On the lower end of the long strip fasten a fairly heavy weight-either a piece of iron or leadweighing at least seven pounds, and let this be in such a position that it hangs on the inside away from the worker, and as nearly as possible under the centre of gravity of the piece of wood supported on the screw-heads. The weight will now keep the dish-support horizontal, and a swinging motion imparted to it will continue to rock any dish which is placed on it for a considerable period. The motion being that of a pendulum, the rocking period may be varied as required by altering the distance between the table carrying the dish and the weight at its lower end. A modification of this appliance is now on the market, and may be obtained from any photographic dealer for a few shillings.

The size of a dark-room is not a matter of great moment, although it should be as airy and lofty as possible, and efficient means should be provided for keeping it ventilated. It should be fitted with a deep sink, preferably of porcelain or lead-lined, and at its right-hand side a bench may be erected on which development or printing processes may be carried out. This bench should be covered with thick glass or lead, or alternatively may be painted so that it can be washed down into the sink and thus kept perfectly clean. Shelves should be placed for the various bottles containing developing and other solutions, and these should be quite close at hand. A position should be apportioned for each bottle, so that the worker who is conversant with the arrangements in the room can find any solution he wants in the dark with absolute certainty. It is a good plan also to have the bottles for solutions of varying sizes and shapes, so that after a time the worker becomes intimate with each one without reference to the label. There is thus less liability of using the wrong solution when the conditions are such that the labels on the bottles cannot be clearly seen.

It is all but useless to affix ordinary gum labels to bottles containing photographic solutions, as the constant use causes them to become quickly detached; it is perhaps better with developing solutions to paint on the bottle a number with some enamel paint, and to have affixed to the wall of the room a sheet of paper on which is set out the various solutions in their order, with their corresponding numbers. 
A supply of porcelain dishes will be required, and these should be deep and of the best quality. The enamel on cheap dishes very soon cracks, and the chemicals that are in use cannot, therefore, be entirely eliminated from the dish as they tend to lodge in these cracks. The dishes should from time to time be thoroughly cleansed with dilute sulphuric acid, or where they have become silver-stained they should be washed with a solution of permanganate of potash. It is above all things essential to keep the dishes thoroughly clean, and at the end of the development of each plate the dish should be washed out, never being left with any solution in it. Nearly all photographic solutions tend to oxidise rapidly, and it is often very difficult to eliminate them entirely from dishes in which they have been allowed to remain for long periods. A supply consisting of three quarter-plate dishes, and two or three about $8^{\prime \prime} \times 10^{\prime \prime}$ for printing operations, will be found sufficient. In addition a fixing-bath is advisable. This consists of a porcelain trough which has a rack in it to enable a good many plates to be placed in it at one time. A fixing solution may be kept in this and used for a number of plates in succession. The plates are put in the trough on edge, and in this position they are fixed much more rapidly and efficiently. The solution should not, however, be used after it has acquired a distinctly yellow tinge.

Two or three graduated glass measures will be needed; one containing two drachms, for measuring small quantities of solution, and two others of four and eight ounces respectively. These should always be thoroughly washed out directly the solution contained in them has been poured out, as these measures are often used for widely varying solutions, an admixture of which would have disastrous results.

As already mentioned, some arrangement must be made to cover the dishes while development is taking place. Nothing is much better than a cheap vulcanite or papier-mâché dish, of a size larger than the one actually in use; that is, a $5^{\prime \prime} \times 4^{\prime \prime}$ vulcanite dish inverted over one of a quarter-plate size will effectually screen the plate from the light of the ruby lamp, or from any stray light which might enter the dark-room.

Draining- and drying-racks may be usefully provided, in which the plates after development and fixation may be placed 
to dry. In placing the plates in such racks it should be noted that they do not dry properly if put too close together; hence a rack holding one dozen plates should not have more than three or four plates placel in,it, to dry at once, and these should be so placed that the greateste air-space possible is allowed between the sensitive films. If dry-plates are stored in the dark-room, care should be taken to ensure that they are kept thoroughly dry, and so far as is possible, in a position in which they are not subjected to any rapid changes of temperature. 


\section{CHAPTER IX}

\section{COLOUR-SCREENS AND PHOTOGRAPHIC PLATES- EXPOSURE}

Colour-screens.-In photographing microscopic objects it will almost invariably happen that a colour-screen is necessary. It is either useful when using achromatic objectives to eliminate that portion of the spectrum for which the objectives are not corrected, and so to obtain a photograph which does not suffer owing to the want of colour-correction of the objective, or it may be necessary to increase or reduce colour-contrast in the object itself. It will very soon be found that although an object may have sufficient contrast visually, it by no means follows that in photographing this object the same contrast can be obtained on the photographic plate.

The elimination of the harmful portion of the spectrum, so far as the objective is concerned, is a comparatively simple matter, and can usually be secured by using a yellow screen which cuts out the blue and violet portions of the spectrum. The part so cut out is, of course, the photographically active portion; but, as in conjunction with such a screen a yellow sensitive plate would be used, this does not result in such great lengthening of the exposure as would otherwise be the case. To increase the colour-contrast of a preparation is not such a simple matter, and the only effective way is to adapt the screen to the particular dye, or combination of dyes, with which the preparation is stained. Colour-screens may now be purchased with almost any absorption spectrum, cutting out, therefore, almost any part of the spectrum which it is wished to eliminate. To purchase such a set of screens is a somewhat costly matter, and many workers may hardly feel justified in incurring such an expense. Fortunately they may be made up 
with comparatively little trouble. The matter may be dealt with in either of two ways. Permanent screens may be made by dyeing either gelatin or collodion films; or, what is much better, liquid screens may be employed, and these may be adapted either in their general or selective absorptive powers to the requirements of the case.

The method of making permanent screens is fairly simple, although perhaps the term 'permanent' is rather misleading, as nearly all aniline dyes are subject to change on exposure to light for any long periods. Whether the screen is made of collodion or gelatin will depend on the dye, which may be soluble either in water or alcohol. If soluble in water, the gelatin film may be made in either of two ways. Some of the best hard gelatin, such for instance as Nelson's No. 1, may be made up into a $7 \frac{1}{2} \%$ solution in water. If the screen is being made in very hot weather, then it is advisable to make the gelatin solution as strong as $10 \%$ to ensure its setting easily. Take a sufficient quantity of the gelatin, and add water to the strength stated: allow the gelatin to soak in the water for some hours, and then place it over a water-bath until it is completely dissolved. The gelatin may now be filtered through glass-wool or cotton-wool and coated on to small pieces of glass, conveniently about three inches square. This is usually done by pouring on to the centre of the glass a pool of the hot gelatin solution, so that about 2 or 3 c.c. of solution are allowed to each piece of the size stated; with a piece of glass rod at once cause the gelatin solution to flow evenly over the glass, and place it to set in a position free from dust and on a perfectly level surface. As soon as it is set, it may be immersed in a solution of the required dye, until it is stained sufficiently deep for the purpose in view. The exact strength of the dyeingsolution is immaterial; the stronger it is, the shorter the time necessary to effect the desired staining. On the other hand, too strong a solution of the dye will often stain too rapidly, so that it is difficult to stop exactly at the right moment. In general with aniline dyes, a strength of one in one thousand will be sufficient. On the completion of the dyeing process, they should be taken out and quickly rinsed in distilled water, then set on edge or in a photographic drying-rack to dry.

An alternative method is to add the dye-solution directly to 
the gelatin, and pour this at once on the plate; the disadvantage of this method is that it sometimes happens on drying that the screen will be found to have a certain amount of the dry dye, which has separated out, on its surface, and it is consequently less transparent than those made by the first-mentioned

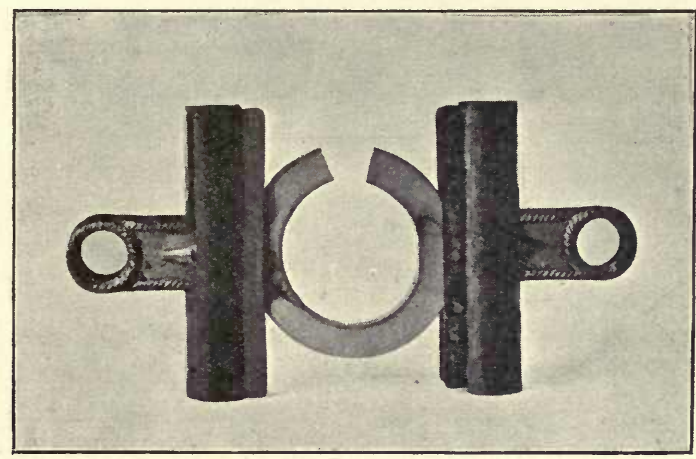

$a$

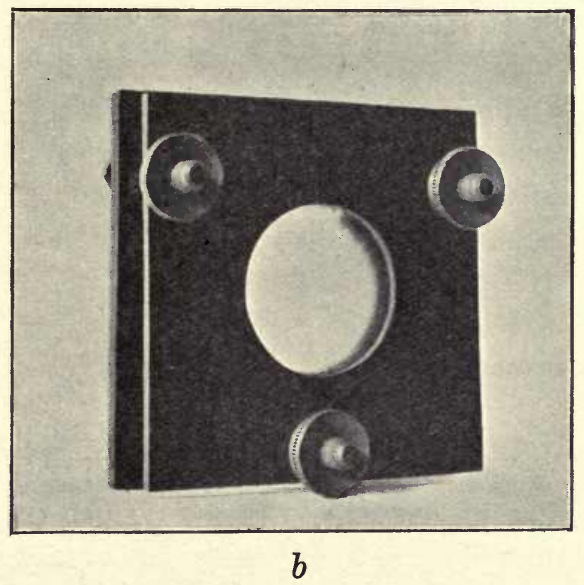

Fig. 64.-Improvised Glass Cell for Screen.

process. This may often be remedied by rinsing the screen under the tap for a few moments and then allowing it to dry again with the same precautions as before.

If a collodion film is required, the dye must be dissolved in absolute alcohol, and this added to perfectly transparent enamel collodion. No ordinary collodion dissolved in alcohol 
and ether will be sufficiently transparent, and it is necessary to procure the collodion described as 'enamel collodion.' A strong or even saturated solution of the dye in absolute alcohol should be used - say, one in a hundred - and a few drops of this added to a small quantity of the collodion and thoroughly mixed. It may then be coated direct on to the glass plates as described for the gelatin films, and allowed to dry. To ensure that the collodion film adheres firmly to the glass an edging of a solution of pure india-rubber in benzole

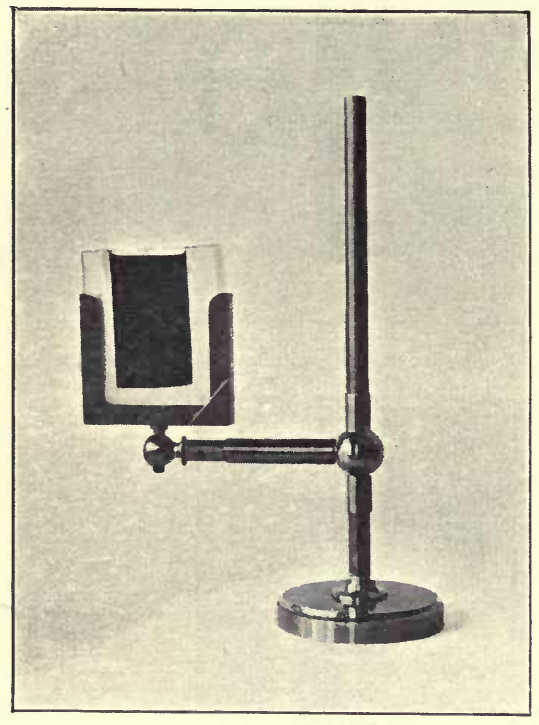

Fia. 65.-Monochromatic Trough. may be applied as in the wet collodion process if need be. None of these dry-film screens transmit quite so much light as a fluid screen, and for this reason the latter is to be recommended.

A cell to contain the solution of the dye, or other liquid colour-screen, may be improvised very simply by taking two pieces of stout glass, preferably thin patent plate, placing between these a piece of india-rubber tube bent into horseshoe form, or a flat india-rubber ring, and clipping the whole together on either side by strong paper clips (Fig. 64a). To prevent bending of the glass, the clpis must engage well over the edge so that the pressure is taken up evenly by the rubber tube between. Such an improvised cell is perfectly efficient, may be taken apart in a moment for cleansing purposes, and its cost is but a few pence, so that a number may be kept at hand ready for use.

Some excellent cells for holding solutions may be obtained from Messrs. Zeiss which are made entirely of glass (Fig. 65), and are actually cemented and fused together, by what is known as Leybold's process. The disadvantage with all cemented 
cells is the difficulty of cleansing them. Where a solution is used which is liable to crystallise out - such as bichromate of potash-the writer has had the side of such a cell forced completely out by the mere force of crystallisation when the solution has managed to get into the cemented joints, even to a very slight extent.

There are many other forms of trough on the market. The one made by Messrs. Watson, and known as Kingsford's trough (Fig. 66), is very convenient. Its construction is
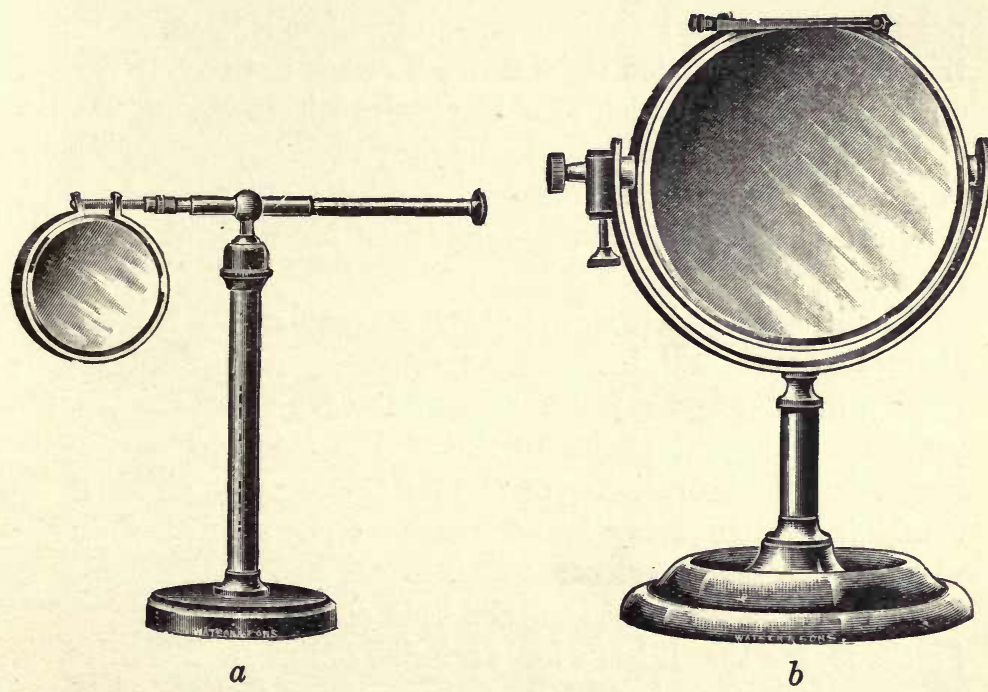

Fra. 66.-Kingsford's Trough for Fluid Screens. (W. Watson \& Son.)

simple, consisting essentially of an outer circle of brass drawn together at the top by means of a screw or screws. This brass circle is lined with india-rubber, and has blocks of india-rubber so placed as to keep in position the circular glass dises or faces. The latter are gripped by the india-rubber when tension on the outer metal band is increased by turning the screws, and an absolutely water-tight fitting is the result. It also has the advantage that by releasing the screws it can be taken to pieces, either to be cleaned or to replace either of the broken glasses, should this be necessary at any time.

Any simple trough could be made by taking two plates of 
vulcanite - or even hard wood is quite efficient-between which are clamped by means of three screws two slips of glass separated by an ordinary india-rubber ring (Fig. 64b). By releasing the screws the glass can be removed and replaced quickly and easily.

The simplest of ready-made filters are pieces of ordinary coloured glass. These may be obtained in a fairly large variety of tints, but their absorption-spectrum is often widely different from what might be expected, having regard only to their visual colour. For ordinary purposes a rather deep yellow, green, blue-green, and blue glass should be chosen. These should if possible be what is known as pot-glass - that is, glass in which the colouring is carried right through and is not merely flashed on to the surface. Such may be obtained from Messrs. Hetley of Soho Square, London. While they may answer their purpose when the very best obtainable results are not necessary, they are open to considerable objection for work of a critical nature.

The illustrations (Fig. 67) are photographs of a few typical absorption-spectra, taken with a spectroscope with a diffractiongrating mounted on a prism, so that the projected spectrum is practically at right angles to the optical axis of the spectroscope. Such absorption-spectra may therefore be obtained by combining an ordinary long-extension camera with a directvision diffraction spectroscope.

The arc-spectrum of iron has been chosen as the standard, for the reason that it has a large number of lines well distributed throughout the whole of the visible spectrum (Fig. $67 a$ ).

Fig. $67 b$ is the same spectrum taken with a piece of green glass intervening. It will be seen that very great general absorption of light has taken place, although the exposure given was about three times that given to the bare iron arc-spectrum, and in addition the light transmitted is not confined to the green portion of the spectrum, but extends without any very well-defined boundary in each direction.

Fig. $67 c$ is taken with the glass known as signal-green. This absorbs nearly the whole of the red end of the spectrum, but it has no particular selective absorption effect on the remainder, and results in considerable loss of light. It is therefore clear that both from the point of view of general loss of light, and from the difficulty of getting glass with a well-defined 
Wave-Length Scale.
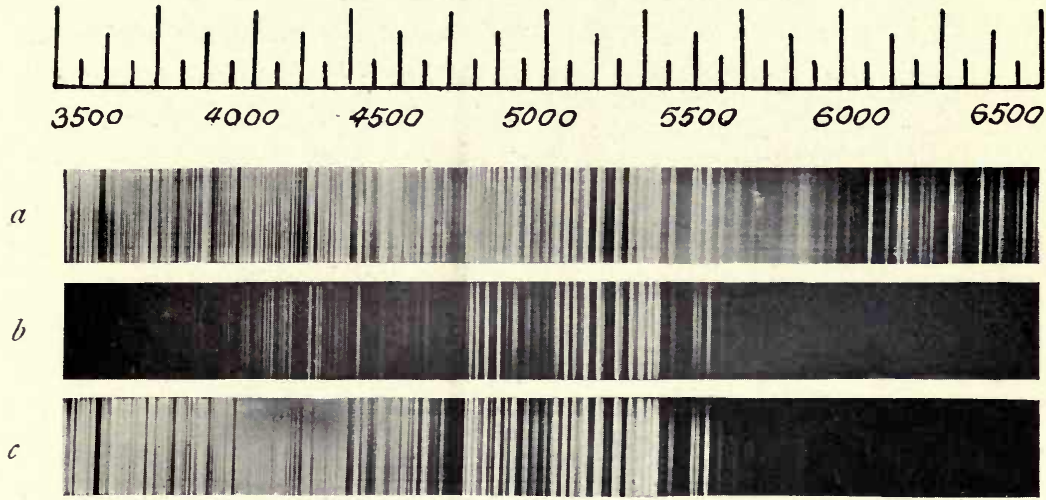

$d$

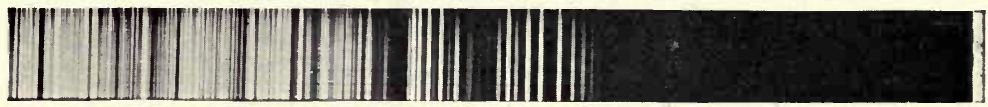

$e$
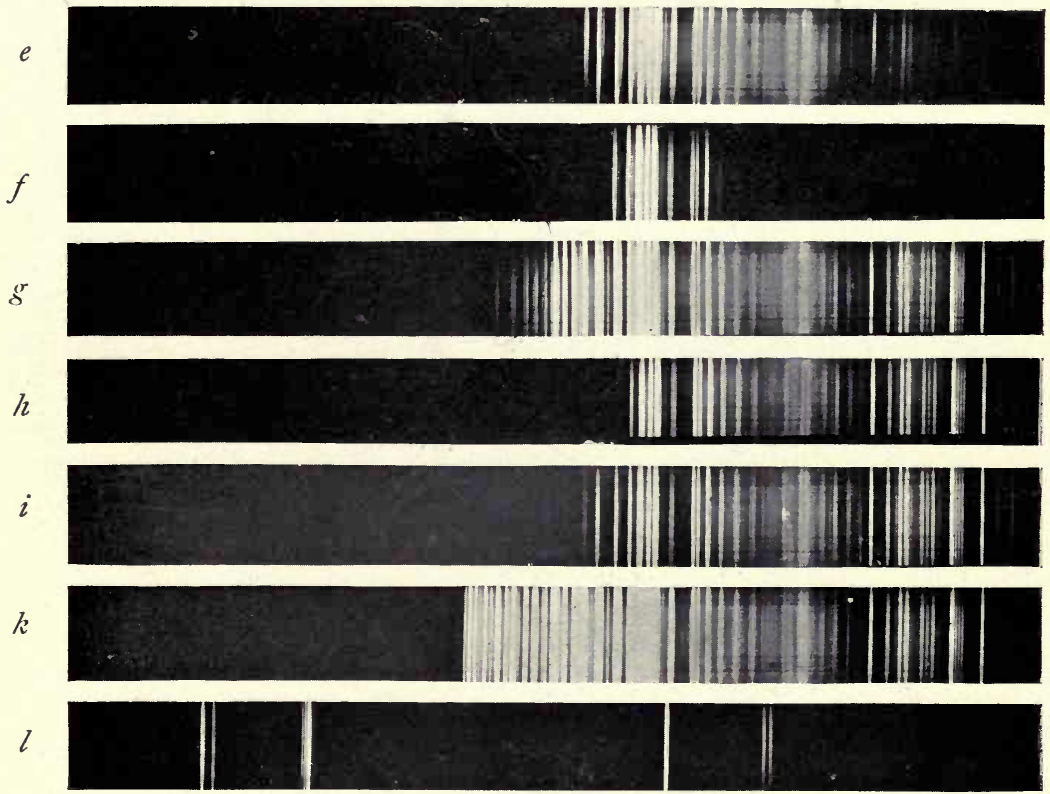

FIG. 67.--Spectra of Various Dyes and Colour Screens. 
absorption-spectrum in any particular region, glass screens are not to be recommended. Recourse must then be had to some of the fluid or stained film-screens made with aniline dyes, or suitable chemical solutions.

The ideal filter would absorb completely all the region of the spectrum that is not required, and would transmit the remainder with no loss of light. It must be admitted that no known filter conforms entirely to these requirements, and even among the best of them general loss of light, amounting to 80 or $85 \%$, is not excessive.

For visual work the best known of all fluid filters is the Gifford F-line screen (Fig. $67 d$ ). This can be made by dissolving malachite-green in pure glycerin, and the strength of the solution must be such that it absorbs all the red end except one narrow band. Immersed in the solution is a thin piece of optically worked signal-green glass, and this must not be thicker than is necessary to absorb the already-mentioned narrow red band; otherwise there is too great general absorption and consequent loss of light. For visual purposes this screen is not to be excelled, but owing to its not having a very sharply defined absorption-spectrum, it is not so generally useful in photo-micrography, except when dealing with diatoms.

The figure shows the light transmitted, and it will be seen that it extends well into the violet end of the spectrum.

In cases where achromatic lenses are being used, a series of yellow filters will be found of use; and, provided that they are not capable of transmitting any of the ultra-violet part of the spectrum, they will ensure a sharp image even if the objective is not photographically well corrected.

A saturated solution of picric acid is one of the best of these. It has a very well-defined absorption-spectrum (Fig. $67 \mathrm{k}$ ), and it will be seen that the whole of the blue-violet and ultra-violet is completely stopped out. It transmits the yellow and green with a minimum of light-absorption.

Of the yellow aniline dyes, the most suitable are filteryellow K, tartrazine, and aurantia (Fig. $67 \mathrm{~g}, h$, and $i$ ). The strength of solution of any of these, assuming that they are used in solution, must depend on the thickness of the cell ; but rather greater efficiency will be secured by using a very thin filter -say, two millimetres thick-and adopting a proportionately 
stronger solution. Such a filter is very conveniently made in the manner described by holding an ordinary rubber elastic band with paper clips between two pieces of glass. A thin rubber band will give approximately the thickness mentioned. When using such a cell the dyes may be kept as saturated solutions in water, and with the above thickness will efficiently absorb the whole of the blue and violet end of the spectrum.

Of green filters there is a very wide selection. The most useful is what is known as Zettnow's filter, in which a saturated solution of copper nitrate is mixed with chromic acid in water. This filter completely cuts out both the blue-violet and the red end of the spectrum. The proportions of the two solutions may be varied so that a greater or less absorption of either end may be obtained at will. The most satisfactory way is to adjust the solution by means of a hand spectroscope until the part of the spectrum not required is eliminated.

Another convenient way of making green and yellow-green filters is to keep at hand a saturated solution of acetate of copper and bichromate of potash; both the solutions should be strongly acidulated with glacial acetic acid. They may be combined in any suitable proportion, and will give a strong visual green or yellow-green filter, the predominance of either colour depending on whether there is an excess of the green solution (acetate of copper), or of the orange solution (bichromate of potash). If on combining these two solutions any precipitate is thrown down, it shows that they are not sufficiently acid, and more acetic acid should be added to re-dissolve the precipitate. Fig. $67 e$ is a photograph of the absorption-spectrum of these solutions combined to give the greatest absorption at the violet end of the spectrum, while transmitting as small a proportion of the red as possible.

Of green aniline dyes, malachite-green, methyl-green, naphthol-green, acid-green, and iodine-green will be found useful. Naphthol-green is visually somewhat yellow-green or emerald, whereas the other four are blue-green. Recently a new dye known as rapid-filter green has been introduced, which results in well-marked absorption in the region of the spectrum between wave-lengths 4500 and 5700. It also has the advantage that its general absorption is not so great as in some other green 
dyes. The strength of solution of these dyes may conveniently be in a proportion of one in one thousand, and the width of the cell in which they are contained should not in this case be less than five millimetres. The absorption-spectra of all aniline dyes vary very largely with the strength of solution; or, what is much the same in effect, the width of cell in which they are contained. A green dye which transmits a specially narrow band, between wave-lengths 4500 and 5000 , is Victoria green, and a solution of the strength mentioned makes a very effcient filter. Tartrazine and acid-green, in separate solutions, make an efficient and nearly monochromatic-green screen (Fig. $67 \mathrm{f}$ ).

For blue filters there is not such a large demand as there is for green or yellow. The most useful chemical solution is ammoniosulphate of copper, and this efficiently cuts off the red end of the spectrum. Most blue aniline dyes transmit a good deal of red, hence they are not generally used except in conjunction with a screen that will cut off the red portion. A solution of night-blue, one in one thousand, transmits a fairly narrow band between wave-lengths 4000 and 5000 , and may be found useful under suitable conditions. Messrs. Zeiss have recently introduced a blue.glass which very efficiently absorbs the whole of the red end of the spectrum; in order to obtain the best result, it should be ordered for and adapted by them to the source of light with which it is to be used.

Methylene-blue transmits a definite band in the red, and must be used in conjunction with a piece of signal-green glass, or some similar absorptive substance which does not allow red to pass.

It is obvious that in selecting colour-screens due regard must be had to the source of light in use. Very weak sources, such as an oil lamp, would only require weak solutions and thin layers to effect the necessary absorption, whereas with an illuminant of high intensity, such as an electric are, or lime-light, a much stronger solution and a thicker layer must be used.

It is almost impossible to give any absolute directions as to the strength of the solutions in view of these variable factors, and also owing to the fact that aniline dyes, even when described by the same names, are themselves variable. A test should always be made with a small direct-vision 
spectroscope to ascertain whether the screen in use is absorbing the particular portion that is not required.

When using a mercury-vapour lamp the conditions are somewhat different to those occurring with any other illuminant. As already stated, the spectrum of mercury vapour is confined principally to three bright lines : one in the yellow-or more correctly, a double line in the yellow-a brilliant green line, and one in the blue (Fig. $67 \mathrm{l}$ ). There is also a violet line which is visually not very evident although photographically very active. It is therefore only necessary to screen off the lines not required, when the remaining one becomes a truly monochromatic source of light. The dyes that will be required to make a series of filters for this light are :-

Filter-yellow $\mathrm{K}$;

Tartrazine ;

Acid-green ;

Methyl-violet, and

Eosin, soluble in water.

To transmit only the double yellow line, two solutions should be made : filter-yellow $\mathrm{K}$ and eosin, each of a strength of one in one thousand, for use in a cell five millimetres thick. To transmit only the green line a solution of acid-green, tartrazine, and filter-yellow $\mathrm{K}$ should be used. In this case the three dyes may be made up into one solution, or rather separate solutions made, and then equal quantities taken of each. This, as a general rule, is an unsafe procedure with aniline dyes, since they are very liable to interact. The absorption-spectrum of the solutions becomes altered in many cases on mixing, or a precipitate may be thrown down.

To transmit only the blue and violet bands a solution of methyl-violet, one in five thousand, is sufficient. Owing to the ease and certainty with which monochromatic illumination can be obtained by means of the mercury-vapour lamp, it is for difficult objects, or faintly stained preparations, of the greatest possible service.

Another method of obtaining monochromatic light is a spectroscopic method in which the light is split up, either by a prism or by a diffraction-grating mounted on a prism, into a continuous spectrum. A convenient apparatus for doing 
this has been suggested by Dr. E. J. Spitta, and is made by Messrs. C. Baker of Holborn. It consists, firstly, of a Nernst electric lamp, or some similar illuminant; a very useful source of light for this purpose is the 4-ampere arc lamp made by Leitz, described on p. 106. The image of the source of light is projected on to a slit by an achromatic lens; the beam is then collimated and passes through a prism with a grating mounted on it, the resulting spectrum being projected on to the sub-stage of the microscope by means of another achromatic lens (Fig. 68).

By moving the microscope from side to side, any portion of

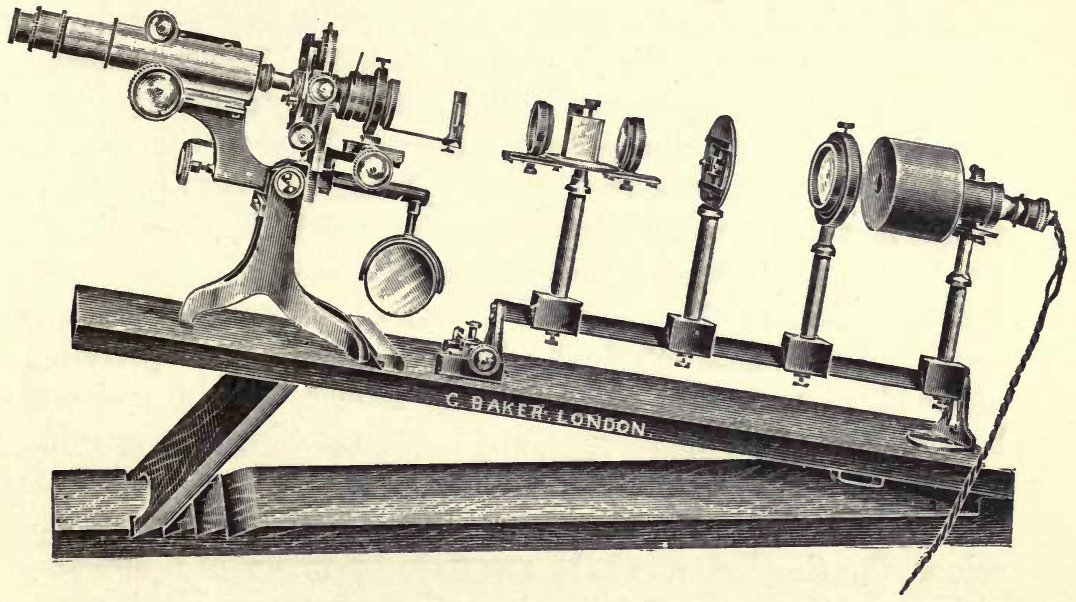

Fra. 68.-Monochromatio Light Apparatus. (C. Baker.)

the spectrum may be utilised as required. Provided that there is sufficient dispersion to ensure that the whole of the field is filled with light of one colour, this method is good enough. But it must be admitted that the loss of light is very great indeed, and in taking photographs the exposure is unduly lengthened. Further, although the light may be visually monochromatic, it is not even approximately of one wave-length, as in the case of the mercury-vapour lamp.

The use of deep-blue or violet filters is of importance not so much for securing colour contrast, as for increasing the resolution of the microscope. Abbe's law for ascertaining resolution 
with a given objective is to multiply the N.A. by twice the number of waves to the inch of the illuminant. It therefore follows that something approximating to twice the resolution may be obtained if light of short wave-length-such as violet-be used instead of, say, the red at the opposite end of the spectrum.

This method is practically only applicable when attempting the resolution of difficult diatoms, or objects with similar recurring structure. In practice, the increased resolution rarely works out at such greater efficiency as theory would indicate, and in general the use of a monochromatic green, or blue-green, light will secure nearly all that is required.

The main object of colour-screens in photographic work is to increase contrast. For example, if we have a faintly stained methylene-blue preparation, and we attempt to photograph it on an ordinary plate without a screen, although the visual contrast is considerable, the resulting photograph, owing to the actinic power of the blue light transmitted, will be disappointing. To obtain a photograph of such a preparation therefore, recourse would be had to the use of an orange-colour or deep-yellow screen. This would effectually block out the greater portion of the blue transmitted, and the image formed would therefore be almost black in appearance, and in the resulting photograph would give the maximum obtainable contrast.

To obtain the maximum contrast effect with any particular colour, the simplest way is to ascertain by means of a directvision spectroscope what its absorption-spectrum actually is, and to use a screen which as far as possible only transmits light in the particular part that the object absorbs. The example mentioned of a methylene-blue specimen with which an orange screen is used, fully carries out these conditions, except that methylene-blue transmits a narrow band in the red; but unless a plate sensitive to the whole spectrum is being employed, this is not a matter of moment.

An example in the opposite direction might be the case of a section or specimen stained with eosin, which would visually be of a pale red or pinkish colour. If this preparation were actually only transmitting red, then a blue screen would be of use; but on observation it will be found that it transmits a great deal of blue as well, so that there are two colours 
transmitted at opposite ends of the spectrum. To obtain the best results in this case, a screen as nearly as possible monochromatic green should be used.

A general rule to obtain the utmost contrast may be formulated for either double-stained specimens, or those stained with dyes which transmit light from widely separated portions of the spectrum. In such cases it is a safe rule to use a screen which transmits light that is intermediate in its position in the spectrum between the two colours it is desired to photograph. Since the spectrum colours occur in the order violet, blue, green, orange, yellow, red, it follows that any two of them may be photographed to secure the greatest contrast in each case by using a colour intermediate between them. Should the two colours transmitted lie together in the spectrum, then the screen used should be as far on either side as possible.

It may be that the object is not to secure the greatest possible contrast, but to reduce it; although the occasions on which this happens are not so numerous. In such a case, instead of selecting a screen which is intermediate in position in the spectrum between the two colours, one should be chosen which transmits the colour in the object which is the most active photographically. If therefore it were desired to photograph a specimen transmitting red and violet, and to reduce the contrast between them as much as possible, a blue or violet filter might be used ; the resulting photograph would then be a reproduction with a minimum of contrast. The latter condition sometimes arises in preparations that are very deeply stained, or which do not transmit very much light.

A very efficient series of monochromatic filters can be obtained from Messrs. Wratten \& Wainwright. These filters are designed to pass special regions of the spectrum ; the regions are as narrow as possible, just overlapping each other, and the transmission in other regions is reduced to a minimum. The absorption in the region of maximum transmission is designed to be as small as possible, but is still considerable; and the makers state that the complete transmission is only about onethirtieth of the incident light even in the region of maximum transmission. These filters do all that is claimed for them, the only point being that in securing such high efficiency a 
considerable general loss of light is unavoidable. It is possible to improvise liquid filters which have somewhat less general absorption, although they may not so efficiently cut out that portion of the light that is not required. The series, as sold by Messrs. Wratten \& Wainwright, consist of seven filters, and with each one particulars are given of the wave-length it transmits. 1

Messrs. Wratten \& Wainwright also issue another series for use with a mercury-vapour lamp. The most important filter of this series is made of didymium glass from the Schott glass-works in Jena. It is somewhat expensive, but it has the highest efficiency of any that are at present known for isolating the green line of mercury vapour.

Sensitive Plates. - There are at present practically only two types of plate that are available for the ordinary worker : gelatin dry-plates, or wet collodion. The former are obtainable commercially ready for use, but the latter have to be coated as required. Wet collodion was formerly the only available process, and it must be admitted that in the hands of experts it gave results that have never been excelled. But the inconvenience of having to coat a plate before each exposure gives an advantage to the gelatin dry plate that to the ordinary worker is irresistible.

In photo-micrographic work the requirements of a plate are that it should give a bright image with as fine a grain as possible; that is, that however fine the detail in the plate, the grain of the silver particles in the emulsion should not be sufficiently large to become evident. In this respect collodion has very much the advantage, as the grain of a collodion plate is much finer than any gelatin one; in fact it partakes more of the nature of a stain than of a definite deposit in the film.

A compromise is possible by using a dry collodion emulsion, and such an emulsion ready prepared is now to be obtained commercially, leaving only the coating of the plates to be

1 An Atlas of absorption-spectra, by Dr. C. E. Kenneth Mees, has recently been published by Messrs. Wratten \& Wainwright. In it the absorptionspectra of nearly all dyes used in staining microscopic preparations are given, together with photographs of the spectra transmitted by a large series of screens that they now make. This work is the most complete on the subject that has yet been issued, and the reader is referred to it if further exact information on the subject is desired. 
performed. After coating they keep for perhaps a week or so, and give extremely good results if the exposure is correctly timed.

For negative-making, however, it will almost invariably happen that gelatin plates will be chosen, and the description of collodion processes will therefore be left until the making of lantern-slides is considered, since it is particularly for the latter that collodion is now best suited and most largely employed commercially.

Gelatin plates, as sent out by the makers, usually have some name attached to them, which is supposed to indicate their speed, although such names rarely convey any definite information to a purchaser. Formerly their speeds were estimated in reference to the speed of a wet collodion plate; but latterly a more exact method of determining the speed has been employed, and although it is still open to discussion whether the description is strictly accurate, it is at least sufficiently so for the purpose in view.

For photo-micrography a plate of medium or slow speed will be found the most satisfactory, since if properly developed, the grain of the image will be as fine as it is possible to get it in a gelatin plate.

Gelatin plates are sometimes described as 'ordinary,' 'special rapid,' or 'extra rapid.' Such plates are usually most sensitive to the blue and violet end of the spectrum - that is, the visually brightest part of the spectrum does not have the greatest photographic action on them. Therefore, in pbotographing preparations in which yellow, green, or red predominate, or in cases where a yellow or green colour-screen is used, an ordinary plate would require abnormally long exposure, and even then the gradation of tone obtained would not faithfully represent the object. To overcome this inequality of sensitiveness, plates are now bathed in a suitable aniline dye, or the dye is incorporated with the emulsion in the process of the manufacture of the plate. The result is that plates may be obtained more highly sensitive to the yellow and green region, in which case they are generally referred to as 'isochromatic.' They may be obtained still further sensitised for practically the whole of the visible spectrum, and are then described as 'panchromatic,' 
Of the suitable isochromatic plates the following may be taken as typical :-

Barnet orthochromatic plate;

Edward's isochromatic, medium rapidity;

Ilford orthochromatic plates ;

The Isolar plate ;

Lumière, both red and yellow, sensitive plates.

Wellington's orthochromatic process plate;

Wratten \& Wainwright's Panchromatic plates.

The above are merely examples of what may be regarded as suitable, and there are doubtless others which will fulfil the purpose equally well. The plate that the writer has found as good as any is the Wellington orthochromatic process plate; it is slow, its orthochromatic properties are all that may be required, and on development the grain is exceedingly fine.

Whichever plate is chosen, it is important that it should be backed - that is, on the side of the glass other than that on which the emulsion is coated, a layer of some black material is applied to ensure that there is no reflection from the back surface of the glass. On any unbacked plates, where there are violent contrasts of light and shade, the effect known as ' halation' is liable to occur. This consists essentially of a spreading action of the light beyond the margin of what is really the properly defined image, and results therefore in a certain indefiniteness at what would otherwise be an edge of perfectly sharp definition. It arises from the light which passes through the emulsion being reflected from the back surface of the plate. This light is scattered over the back of the emulsion, resulting in some photographic action where it is not wanted. If the reflection of the light from the back of the plate is prevented, the spreading action is very much minimised. Plates may be commercially procured ready backed, so that it entails no extra work except that the backing generally has to be removed either before development or at an early stage in the process.

In photographing objects with very fine detail in them, unless the plate is efficiently backed, there is considerable loss of detail; and this, in some cases where the contrasts are very great, makes it almost impossible to obtain certain results except on backed plates. 

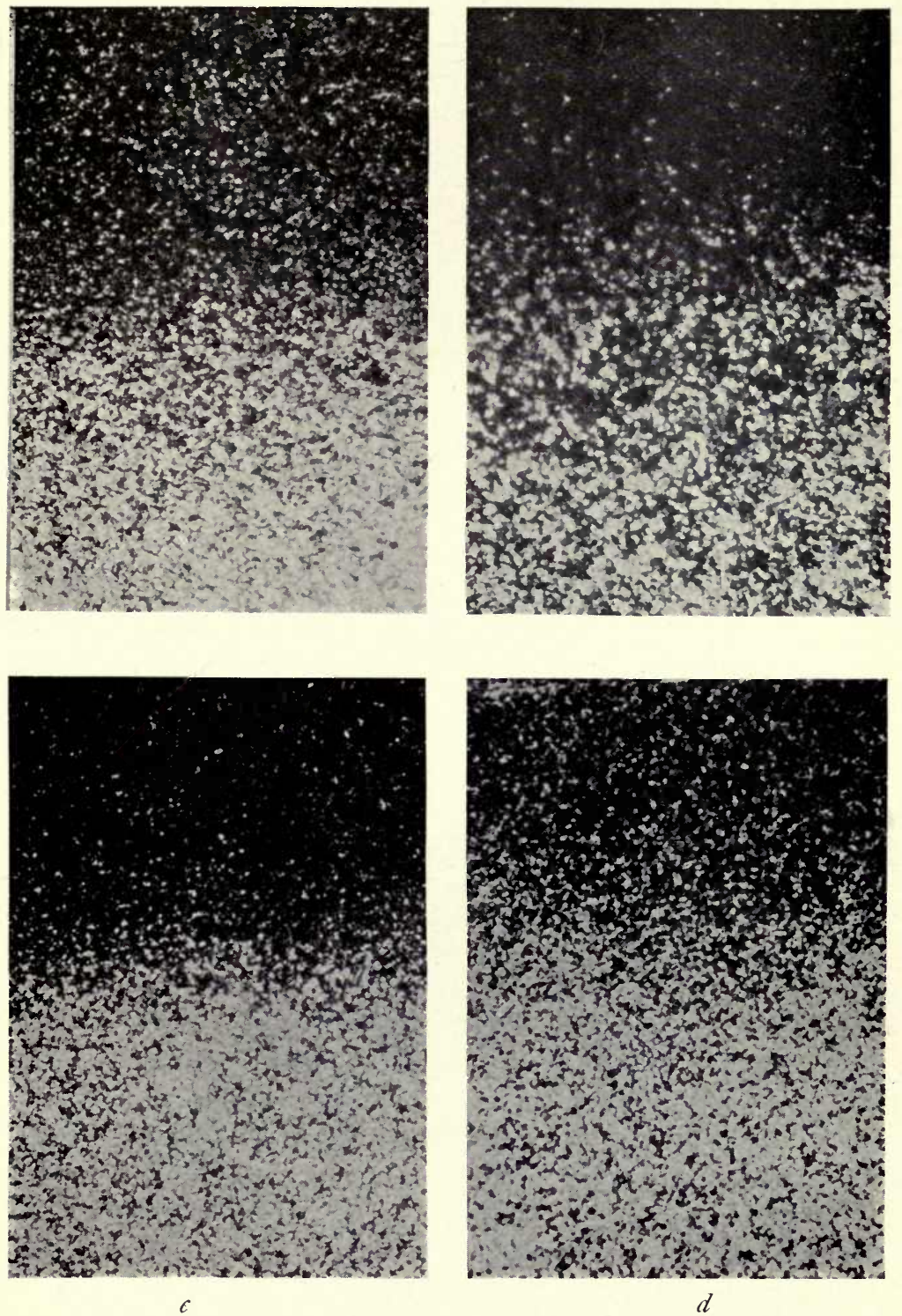

FIG. 69. - Photographs of Grain of Exposed Photographic Plates. $\quad \times 240$.

$a$, Slow backed Plate.

$b$, Fast Plate unbacked.

$c$, Slow Plate Farmer reduced.

$d$, Fast Plate persulphite reduced. 

An alternative method of preventing halation is provided in the Isolar plate. In this a red-coloured film is interposed between the emulsion and the surface of the plate, so that no actinic light passes beyond the lower side of the emulsion-film. The red colour has to be discharged during the process of development, and the makers of the plates give full instructions how to do this. While it would appear in theory that this method is superior to any method of ordinary backing, yet in practice it sometimes happens that it is difficult to discharge the colour thoroughly.

Fig. 69 shows photo-micrographs of a slow backed plate $(a)$ and a fast unbacked plate (b). The magnification is 240 diameters, and it shows quite clearly the difference between the size of the grain in a fast and a slow plate. The object photographed is one of the lines in an Abbe test-plate, which visually appear in the negative to be perfectly defined. The dark and light portions in the illustration show that even what appears to be a well-defined line is not actually perfectly sharp; but in the case of the slow plate the result is much better than in the fast one. The difference in the grain is at once obvious; but in the case of the fast plate this was also unbacked, so that the spreading action of the light as well as the larger grain is shown up.

In actual practice, although it has been recognised that the results to be obtained on very slow plates are superior to those on fast ones, yet for some hitherto unexplained reason it generally happens that a plate of medium rapidity gives the best result. In a paper recently read before the Royal Society by Dr. Kenneth Mees, ${ }^{1}$ on the resolving power of photographic plates, it was shown that for rendering of fine detail, more particularly in the applications of photography to spectroscopy, a plate of medium rapidity might be expected to be the most suitable. This result is dependent not entirely on fineness of grain, but on light-diffraction or reflection within the film and between the particles of silver themselves in the emulsion. It is recommended that those who purpose doing work of a highly critical nature-especially with objects in which there is fine recurring structure-should refer to, and carefully read, this paper.

1 British Journal of Photography, December 24, 1909. 
It was also shown that diffraction and scattering of the light becomes less as the wave-length of the light used increases. It therefore becomes even more necessary to use a suitable plate when violet or ultra-violet light is being used as an illuminant.

Exposure.-Having set up the microscope and properly adjusted the optical system, it is then necessary to determine what exposure shall be given. It must be admitted that this is not an easy matter, and that it is one the determination of which with varying objects can only be arrived at as the result of experience. No definite rules can be laid down, but in general, exposure depends on three chief factors :-

1. The actinic value of the source of light;

2. The speed of the plate;

3. Magnification.

Other factors may have to be taken into consideration in determining the exposure-particularly the character, colour, and density of the object and also the colour-screen used. The latter is, however, usually a known constant factor, so that the necessary allowance can always be made for any given screen.

All so-called methods of determining exposure are based on the assumption that the exposure is correct when a sufficient light-action has taken place in parts of the projected image in which there is no light-obstruction. This assumes that an uncoloured object is in the microscope which is delineated entirely by perfectly transparent and perfectly opaque areas. The exposure in such a case would, of course, be correct if sufficient light-action had taken place in the transparent parts; but, unfortunately, the number of such objects is extremely limited, and, in actual practice, the conditions are rarely exactly complied with.

A very simple appliance for estimating the relative intensity of illumination at the position of the focussing-screen is the Warnerke or Chapman-Jones sensitometer, the use of which was first suggested by Dr. Bousfield. The appliance as sold commercially is really intended for determining the speed of sensitive plates, and consists of a glass plate divided up into a number of squares which differ in transparency to light. Each one is numbered and bears a definite relationship in its light-transmitting power to the square preceding it; the numbers run from one to twenty-five with regularly 
decreasing transparency. It follows that if the sensitometer is placed against the ground-glass focussing-screen of the camera, the image being set up and illuminated, it will be possible to read the numbers on the squares up to a certain point. A number will be reached after which it becomes difficult to decipher further. The number that can be read without difficulty may then be taken to indicate relatively the value of the light under those conditions.

To obtain more exact indications from the sensitometer when used for exposure purposes, it will be necessary first to make some trial exposures. It should at once be said that it is hardly a suitable method for use with high-power illuminants, as with these the range of densities on the screen is not sufficiently great, and it will probably happen that the densest square of the sensitometer can be read with ease. This may be partly overcome by interposing one or two thicknesses of grey glass between the sensitometer and focussing-screen, so that the power of the light is lessened by a definite amount ; the same glass must of course be used on every occasion when it is desired to estimate approximately the power of the transmitted light. In practice it will be necessary to make a number of trial exposures. Having determined the correct exposure for any one number it is then easy to determine it for any other visible number. As an approximation the following short table may be regarded as an indication of the method as it would apply in actual work with a source of light of moderate power, such as acetylene:-

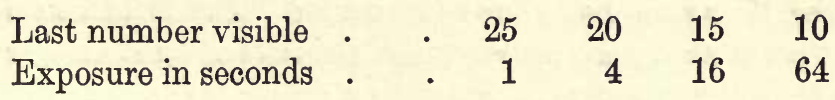

and so on, in regularly progressive increase.

It must clearly be understood that the use of the sensitometer is but an approximation, and it must only be used, and what it indicates must only be acted on, after due consideration of all the other factors that require to be taken into account.

It is obvious that the working N.A. of the objective and of the sub-stage condenser would greatly influence the question of exposure, as on these factors the light-transmitting power of the optical combination depends. It has been suggested that exposure might be determined solely in relation to this 
actual working N.A., and with this object in view a complete table, basing exposures solely on this factor, was laid before the Royal Microscopical Society on October 16, 1907, by Mr. Alfred Latherby. A full report of his paper is to be found in the Journal for that year. While this method would appear to work very well with a range of objects that did not present any considerable colour or contrast difficulty, it would be of little assistance in cases where widely varying objects had to be dealt with. The difficulties of exposure are most in evidence, of course, to the beginner; to such the method may be commended as enabling him in the course of his work to obtain a series of records that would form a basis for approximate estimations. The method works out in practice exactly on the lines for determining the illumination of any area by an illuminant at varying distances-that is, that the intensity of illumination varies as the square of the distance. Hence with a N.A.- say of 1 -and a magnification of 100 , an object would require one-fourth the exposure that it would with a magnification of 200 , and one-sixteenth with a magnification of 400 , assuming that all other factors remain the same. It is not a difficult matter, where the working N.A. is known, to formulate a table from a few trial exposures which will suffciently indicate what is needed with varying magnifications.

The weak point about the method is that it is not always easy to estimate exactly the working N.A. To remedy this and supplement the method, Dr. Duncan J. Reid has suggested that this factor may be determined by measuring the diameter of the Ramsden disc above the ocular. The Ramsden disc is that point above the ocular where the rays of light after emerging from the eye-piece converge to a point; and the diameter of this circle varies with the aperture of the objective and the power of the ocular. The greater the magnifying power of the latter, the smaller does this disc become. If this small disc is projected on to a transparent millimetre measure, and by means of an ordinary hand-magnifier its size is read off - say in millimetres and tenths-it is possible to determine its diameter directly. Half this diameter should be multiplied by the magnifying power of the ocular (which is usually known), and the total divided by the focal length of the objective; the result gives the N.A. of the objective in use. As an example, 
assume that a 4-mm. objective, with an ocular magnifying four diameters, is being employed, and that the diameter of the Ramsden disc of the ocular, obtained by direct measurement, is $1 \mathrm{~mm}$. This, multiplied by the power of the ocular (4), gives $4 \mathrm{~mm}$. as the diameter of the objective beam ; half of this, $2 \mathrm{~mm}$., is the semi-diameter of the beam, which, divided by 4 , the focal length of the objective, gives 0.50 as the N.A. in use at the time. It is, of course, necessary in this case again to make some trial exposures, and to determine with a given illuminant what is the correct exposure for a colourless object; from one or more trials a complete table may then be worked out which would give the exposure under varying magnifications.

This method appears to be the most scientific that has yet been suggested for estimating exposure; but here, again, the same objection applies - that it is left to the discretion of the worker to determine what allowance shall be made for colour or density differences in his object. With the same illuminant, however, and with one that can always be worked at the same intensity, it would appear to be of considerable service. It is based on the assumption that critical illumination is being used, and is not applicable if this condition is departed from.

If any determination of the actinic value of the projected image is to be made, then the most practical method is to employ either a Wynne or Watkins exposure-meter. This is the method employed in ordinary pictorial photography, and under proper conditions may be used with considerable accuracy even in photo-micrography. It depends on observing the actual effect of the light on paper coated with a sensitive emulsion. The paper is allowed to darken until its tint exactly matches that of a tinted standard supplied with the appliance. For photo-micrography the type that is usually made for estimating the exposure when doing ordinary photographic enlarging, is the one to employ. Using this in conjunction with a source of light of moderate or high powersuch as lime-light or the electric arc-the actinic value of the light may be determined with accuracy. In this case also some trial exposures would need to be made, and from such trial exposures a table may be drawn up for future reference. The 
actinometer must be exposed to the light in exactly the same position that the image will occupy on the screen, the groundglass screen in this case being removed so that the light is projected directly on to the exposure-meter. In the watchform of meter, such as Wynne's, there are two standard tints, to either of which the sensitive paper may be allowed to darken until it matches. The lighter one is the more convenient to use with artificial light as the time occupied is shorter, being in fact one quarter of that necessary to acquire the darker tint. The exposure necessary will vary directly as the time indicated by the meter; so that one or two correct exposures having been made experimentally, a record may be kept and future exposures varied in accordance with the meter indications. It will nearly always happen that one source of light will be adopted, and variation from this will rarely occur. Should, however, a change of illuminant become advisable for any reason, then the following may be an indication of the relative actinic value of some well-known illuminants. Taking an efficient type of oil lamp as a standard - that is, one which has a round wick and gives a fairly white flame-its power in relation to the electric arc is roughly represented as follows :-

An arc lamp taking 7 amperes equals approximately 39 oil lamps.

"

It will, therefore, be seen that the electric arc increases in actinic efficiency as the current rises, which is entirely in accordance with its increase of visual luminosity.

An acetylene lamp, in relation to an oil lamp, is area for area of flame of about five times its intrinsic brilliancy. Limelight, using a blow-through jet, would be from seventeen to twenty times as actinic as the oil lamp; and, using a mixed jet, from twenty-seven to thirty-five times. The comparisons in these cases are area for area of flame, and do not of necessity represent the relative total illuminating power. The Nernst electric lamp varies according to voltage; but a 100-volt lamp may be estimated at approximately ten times the actinic value of an oil lamp. With higher voltages the Nernst lamp increases somewhat in efficiency, so that at 240 volts the increase will approximate to thirty times. These estimations are but 
indications of the relative value, and would depend on the manner in which the illuminants are worked to ensure getting the greatest efficiency.

The speed of plate will, within wide limits, also influence the time of exposure; but here again it will be wise for one make and variety of plate to be adopted and adhered to. The speed-value of the plate is usually indicated by the makers on the box; in fact some makers, particularly Messrs. Wratten \& Wainwright, now determine the speed-value for each batch of plates sent out, so that marked speeds may be taken a, indicating very exactly what is the actual speed of the plate. Formerly, exposure had to be effected with a considerable degree of accuracy to ensure that a negative with proper gradation should be obtained; but latterly, plates have been made which admit of considerable latitude in this respect. In microscopic work, as in ordinary photography, it may be regarded as a safe rule to expose for the deepest part of the subject-that is, expose for the shadows. But in developing, regard must generally be had for the high lights ; extreme density must not be obtained in the high lights and detail in those parts must not be obliterated before that in the shadows is brought out. Exposure should be full in any case; it is better to over-expose than to under-expose. An under-exposed plate is useless, and may be regarded as such at once without wasting any time in endeavouring to save it; but an over-exposed plate, in view of the latitude that now exists, may be suitably developed and result in a good negative.

No indication can be given as to the increase of exposure necessary when using colour-screens. For ordinary photography the yellow screen which is supplied commercially increases exposure by daylight from four to ten times, depending on its depth. Should such a screen be used, the same factor will to some extent apply, but will vary according to the illuminant. Where screens of varying colours and varying absorbing power are used, the only practicable way is to determine by trial exposures what the actual increase really is. A record may then be made of what this amounts to, and allowance made for such increase when exposing. With some screens the increase is not very great. Take for instance the Gifford F-line screen, which is visually not very 
bright; owing to the fact that it transmits a good deal of the actinic portion of the spectrum it may only increase exposure from four to five times. On the other hand, with deep screens of a yellow-green variety, an increase of forty to fifty times is by no means unusual.

In general it may be said that a systematic series of exposures made on objects presenting no colour difficulties, with the source of light generally in use and with a given plate, will afford more information-especially if records are kept of the conditions under which the photographs are taken-than any amount of instruction. 


\section{CHAPTER X}

\section{PHOTOGRAPHIC PROCESSES}

Negative Development.-The method of developing photo. micrographic negatives does not differ from that adopted in ordinary photography, and the reader is recommended to refer to one of the many text-books on pure photography that exist if more detailed instructions are required. Particularly if this is the first attempt at any photographic process, recourse must be had to a simple text-book.

The process in general is as follows. The plate, after exposure, is removed from the dark-slide, and is at once without any preliminary washing put into what is known as the developer. This acts on the plate, and the result of the exposure becomes evident in a shorter or longer time. When development is completed, the salts of silver unacted on and remaining in the film have to be removed; this process is known as fixing the plate. After fixation is complete, the plate has to be washed for a shorter or longer period, according to the thickness of the film of emulsion on it, until the fixing solution is completely eliminated from the film. The plate is then put on edge in a position free from dust, to dry, and after drying is ready for any printing operation.

The number of developers is practically unlimited, and it is best at once to realise that there is very little virtue in any particular developer, but that it is rather a question of the manner in which the developer is used. Assuming that exposure has been correct, development may be conducted with comparative ease, and, provided a sufficiently long time is allowed for the developer to act, a satisfactory negative is likely to result. There are three methods now used for ascertaining 
when development has proceeded for a sufficient and proper time :-

1. By direct observation of the plate during the development;

2. By time;

3. By that known as the 'Factorial' method.

In the first case the developer is allowed to act until all the detail that is required to appear in the resulting photograph can be well seen on the plate, the method therefore being one in which a certain amount of experience and careful observation are essential. The time-method eliminates the personal factor to a large extent, the plate simply being put in a given solution at a certain temperature for a given length of time, and when such time has elapsed the plate is removed from the developer, washed, and at once fixed.

In the 'Factorial' method the point to determine is the time that elapses between putting the plate into the developer and the first appearance of the image. This time is multiplied by a certain factor-this latter depending on the developer being used-and the total time to be allowed is then the time that the image takes to appear, multiplied by the development factor. In this case again, therefore, the personal element does not come in, the method being more or less mechanical. The majority of workers, however, will in all probability at least at first confine themselves to the first method-in which the image is directly observed-to determine when development has proceeded far enough ; and for photo-micrographic work it is more than probable that this is the most suitable method. It usually happens that it is not so much a question of bringing out a picture with perfect gradation, as of obtaining one in which there is considerable differentiation between the parts of an object, and for this purpose the observational method is unquestionably the most satisfactory one.

It must not be overlooked in all photographic operations, that the temperature of the room in which the work is being carried out, and of the solutions, is one affecting the result very largely; particularly does it affect the time that the various operations take to perform.

The developers that will be found useful for negativemaking are the following :- -

Pyrogallic acid, hydrokinone, metol, amidol, ortol. 
There are a considerable number of other substances used as reducing agents; but these, or one or two of them, will be found to perform all that is required. Every maker places in each box of plates sent out some formulæ with which the particular plates may be developed, and it is obvious that if these are followed they may be expected to give perfectly good results. On the other hand, nearly all plates now have a certain amount of latitude, and may be expected to give quite as good results with standard solutions, in which there are suitable amounts of developer.

To reduce the number of solutions required as far as possible, the following four may be made up :-

Solution No. 1.

Pyrogallic acid . . . 8 grammes.

Soda metabisulphite . . 4 grammes.

Water . . to make up to 1000 c.c.

Solution No. 2.

Sodium carbonate . . . 50 grammes.

Sodium sulphite . . . 50 grammes.

Water . . to make up to 1000 c.c.

Solution No. 3.

Metol

Hydrokinone

4 grammes.

Potash metabisulphite . . 4 grammes.

Water . . to make up to 1000 c.c.

Solution No. 4.

Potassium bromide . . . 10 grammes.

Water . . to make up to 100 c.c.

The advantage of adopting these solutions is that they may be employed with any plate, with which the writer at least has had any experience. There is the additional advantage that by using them in suitable combination with one another, the same solutions may be used for developing either negatives, lantern-slides, or for printing on bromide paper. The pyrogallic acid and sodium carbonate solution will be found in many respects most suitable for negative-making, and to compound a solution, equal parts may be taken of No. 1 and No. 2 .

Solution No. 4 is a restraining solution only, and in cases where the negative is known to be somewhat over-exposed, or 
where particularly hard contrast is desired, then from five to ten drops of this solution may be added to each ounce of developer.

For lantern-slides or bromide-printing, solution No. 3 may be substituted, and in this case equal parts should be taken of solutions Nos. 2 and 3. But this will be referred to again later when treating these particular subjects. All these solutions keep well for at least some months; but they should in any case be kept in well-stoppered or well-corked bottles. It is better not to make up a larger amount of solution than is likely to be used in the course of a month or two.

In cases where a negative is apparently over-exposed, the amount of solution No. 1 may be increased, and that of solution No. 2 decreased somewhat; and the reverse applies in cases of under-exposure, No. 2 then being in excess, and No. 1 somewhat reduced. In cold weather these solutions will be found to act considerably more slowly than in hot; in fact, when the temperature is low it will generally be found advisable to have a somewhat more energetic developer, as the developing action, apart from its speed, appears to be somewhat retarded. The metol-hydrokinone formula may also be used for negative development, and in cases where softness of gradation and full detail is required, it will be found quite suitable.

When development is completed, the plate should be washed for a few minutes under the tap, and then immersed in a fixing solution composed of 200 grammes hyposulphite of soda, and water to 1000 c.c. The exact strength of this solution is immaterial; but the plate should be allowed to remain in it until all whiteness, as seen from the back of the plate, completely disappears. A fresh solution should be used for each batch of plates, and it is better to leave the plate in the solution for several minutes after apparent complete fixation to ensure that no unaltered silver remains in the film. Incomplete fixation is sure to result in the appearance of markings on the plates at a later stage, or to cause some general deterioration.

After fixation is completed, the plate should be washed thoroughly in running water for about an hour, and if washing is carried out efficiently this time will suffice. The plate may then be placed on edge or in a suitable rack to dry, and this should be done in a place as free from dust as possible. 
Particularly during the operation of developing, and also, if possible, during fixation, the plate should be frequently rocked to ensure even action of the solutions, or one of the mechanical rockers, already described, can conveniently be used.

To develop a quarter-plate, fully one ounce of developer should be used. It is important to see that the plate is well covered by the solution, otherwise air-bubbles may attach themselves to the plate, and cause clear transparent spots to appear in the negative.

However safe the dark-room light may be, it is advisable to cover the plate during development, and in all operations to shield it as much as possible from light. For this purpose it is convenient to have either a dish of a size larger than the one in use to invert over the one containing the plate, or a cardboard plate-box may be conveniently used to cover the dish.

When pyrogallic acid is used, it is as well to have fresh developer for each plate. Rather rapid oxidation occurs with this developer, and if it is used more than once, considerable staining of the plate occurs. With the other developers this is not the case, and they may be used two or three times, the only effect being that they become gradually slower in action.

In the case of a plate which is obviously not as it should be, it is not always easy for a novice to judge whether it is overor under-exposed. In general, the over-exposed plates come up very quickly when placed in the developer, and there is a lack of contrast in the resulting negative. With under-exposure, although there may be ample density in parts, there will usually be some areas in which there is a total lack of detail. Further than this, it is very difficult to indicate the differences between under- and over-exposed plates, since the effect will vary so largely with different objects. The over-exposed negative is often thin, although it possesses ample detail in every part, and in this case it may be efficiently dealt with by the subsequent operation of intensification. The under-exposed plate can rarely be dealt with at all, and unless the negative is one that cannot be repeated, it is far better to expose another plate at once.

The appearance of a number of small spots in the negative 
indicates the presence of dust. This is now rarely on the plate when supplied by the makers, and is probably the result of dust settling from the dark-slide or camera itself. Both before exposure and before development the plate should be sharply tapped on its edge on the dark-room bench, to dislodge any dust particles attached to it. This is a better method than that of using the dusting-brush, or any such appliance, as these often only add to, rather than remove, the adherent dust.

With developers, as with plates, it is far better to adopt one standard formula, and keep to it, mastering its peculiarities and possibilities thoroughly.

In cases of under-exposure, where development has to be forced by using an excess of alkali, care should be taken to stop the process before general fog appears. In any case the amount of the alkali solution permissible is usually not greatly in excess of the normal amount, and an increase of about ten per cent. in the quantity is probably the limit. Where intensification has to be effected, and the negative is somewhat under-exposed and forced in developing so that fog results, the latter should be removed by a weak reducing-solution before intensification is attempted. Many workers, who only conduct photographic operations at irregular intervals, are troubled by the deterioration of their solutions. This may be dealt with in one of two ways. The solutions may be made up in the quantities mentioned; but instead of storing them in large bottles, they may be stored in several small ones, which should be tightly corked or sealed down to prevent the access of air. One may then be opened and as far as possible used up as required. In any case the keeping quality of solutions is considerably increased by well boiling the water before making up, so ensuring that there is the least possible amount of air remaining in solution. An alternative method, and one that is now easily available, is to use the tabloid developers made by Messrs. Burroughs Wellcome \& Co. Almost any developer can be obtained in tabloid form, and there is the advantage that only a sufficient quantity is made up at a time to deal with the plates to be developed. The solutions are therefore fresh, do not suffer from deterioration, and work under their best possible conditions. The only point to be urged against them is the time taken in dissolving the tabloids of each kind when a plate 
has to be developed, and the slight additional cost of the tabloids as compared with chemicals bought in bulk.

In some cases of under-exposure, when it is impossible to secure another negative and forced development is therefore absolutely necessary, a small quantity from 10 to 20 drops per ounce of developer of a $10 \%$ aqueous solution of metol may be added to a pyrogallic-acid developer. This method is preferred by some workers to that of adding more than the normal quantity of alkali solution, as there is less liability of the plates being fogged when development is greatly prolonged.

Intensification.-Some negatives, through insufficient development, may not be dense enough to yield a bright print. An example of this is seen in Plate VII, Fig. 2. This specimen was very faintly stained, and in spite of the use of colourscreens sufficient contrast was not obtainable to yield a bright print. The illustration is taken from the untreated negative; but had intensification been resorted to, a more brilliant result would easily have been secured. This is a case in which such after-treatment is clearly indicated, and it will often be found when dealing with insufficiently stained bacteriological or similar preparations that it is difficult to get a negative with sufficient contrast, unless afterintensification of the negative is resorted to.

In some cases where a negative is considerably overexposed, and is thin, slight reduction followed by intensification will very much improve the result.

There are many methods of intensification available; the following will, however, generally be found sufficient:-

Uranium Intensification.

Solution 1.-Uranium nitrate Water

23 grammes. to 1000 c.c.

Solution 2.-Ferricyanide of potassium 23 grammes. Water . . . to 1000 c.c.

The intensifier is prepared by taking equal parts of these two solutions and adding 2 c.c. of acetic acid to each 50 c.c. of the resulting solution. The plate must be thoroughly well washed after fixing, as it is essential that it shall be perfectly free from hyposulphite of soda, and it is then immersed in the above.

After sufficient intensification has been effected, the plate must be placed in several changes of distilled water until the 
yellow stain has disappeared. It must not be washed in running water, or the image is again reduced. If any stain remains after several successive washings, a weak solution of ammonium sulphocyanide may be used to remove it. This intensifier, under suitable conditions, is perhaps one of the most useful, as the possible increase of density is very great; but unfortunately the results are not entirely stable.

Another method of intensification, and the one most often used, is as follows. The negative is bleached in a saturated solution of bichloride of mercury to which about $\frac{1}{2}$ c.c. of hydrochloric acid has been added to each 100 c.c. of solution. The negative is allowed to remain in this until it is bleached right through to the back, and is then taken out and thoroughly washed. It may be blackened by immersion either in weak ammonia, $10 \%$ solution of sodium sulphite, or any alkaline developersuch as pyro-soda or metol-hydrokinone, already mentioned. The blackening by ammonia gives the greatest amount of intensification, but the results are not so stable as with either of the two last mentioned. Intensification with mercury is in some cases very useful, but it results in an increased granularity of the film; in some cases this is so marked that it shows definitely in the print. A more permanent method, and one that gives a finer deposit, is after bleaching with the bichloride of mercury to immerse the negative in the following solution :-

Saturated solution of potassium oxalate, 6 parts ; Saturated solution of ferrous sulphate, 1 part.

The ferrous sulphate must be added to the ferrous oxalate, otherwise a yellow precipitate is thrown down; but the latter may be re-dissolved by the addition of a small quantity of the ferrous oxalate solution. The negative is immersed in this until blackened throughout, and is then well washed and dried. If the increase of density is not sufficient, the operation may be repeated again and again; but between each a thorough washing of the plate must be carried out.

Reducing.- - In cases where excessive density has been obtained, usually by over-development, the process of reducing may be resorted to. In the class of work under consideration, that known as Howard Farmer's Reducer is perhaps the most useful. It has the particular property of reducing the thin 
parts of a negative without proportionately reducing the denser parts. The result is that in negatives in which great contrast is required, a better result may often be obtained by very full development and by then applying the reducer, the effect being that the dense parts remain practically unaltered, while the thin parts may be completely dissolved out. The solution is made by taking some fresh fixing-solution and adding to it from ten to twenty drops per ounce of a saturated solution of ferricyanide of potassium. It is sufficiently near to estimate the necessary strength by the colour of the resulting solution, as it should be of a pale-yellow colour. It is rapid in action, and should therefore be used rather weak.

In cases where reduction of the dense parts rather than of the thin parts is required, the following solution should be used :-

Ammonium persulphate . . 25 to 50 grammes. Water. . . . . to 1000 c.c.

A fresh solution must be made up at the time of use, preferably in distilled water, and a drop or two of sulphuric acid added to each 100 c.c. The negative must be thoroughly washed before the reducer is applied, and rather before the reducing action is complete must be immersed in a $5 \%$ solution of sodium sulphite. The latter is necessary to ensure the stoppage of the action at the right moment, otherwise the process continues after removal from the persulphate solution.

If very considerable reduction has been effected, it is advisable to put the plate into a fresh fixing-bath before washing, and finally drying.

These two reducers will probably be found sufficient for any contingency that may arise, and it is important to realise the great difference which exists between them in their mode of action : the one acts in a manner that increases contrast, and so is useful where black-and-white results only are aimed at; the other tends to reduce contrast, and may be used in cases where there are wide tone-differences in the negative which renders it difficult to print out detail in the densest parts.

Printing Processes. - A satisfactory negative having been obtained, the next process is to obtain a print on paper from it. To the ordinary photographer there are a large number of processes available; but in photo-micrographic work, where 
the artistic side need not be considered, there are only two in general use :-

1. Printing by daylight on gelatino- or collodio-chloride printing-out paper.

2. Printing by artificial light on some type of bromide paper.

Either of these methods gives a print in which the image is on the surface of the paper and not in its depth, so that the maximum amount of detail is brought out.

The first process is that usually referred to as printing on P.O.P. The paper may be bought of the required size, and is placed in a printing-frame with its sensitive surface in contact with the film-side of the negative. The paper should be placed in position in the frame in a dull light and then placed in bright daylight to print. The time occupied in printing depends on the state of the light, and the time of year, and may vary from half an hour to a day, or even more in winter.

In cases where printing is greatly prolonged, care should be taken to see that the negative and paper are well protected from damp, as otherwise staining of the negative, which it is practically impossible to remove, may result. The resulting print can be modified to some extent by the brightness of the light in which it is printed. In dealing with a very dense negative it is sometimes permissible to print in direct sunlight ; whereas a very thin negative may be somewhat increased in brilliancy by printing in as weak a light as possible. The printing process should be carried somewhat farther than appears necessary, as considerable reduction of the image occurs in subsequent operations.

The above operation having been completed, the next thing is to tone the print, thereby altering its colour, which may be ultimately almost any shade from brown-red to blue-black. The toning process is best carried out by the solutions recommended by the makers, and the formulæ for these are always enclosed in each packet of printing-paper. Practically, however, all papers of this type may be toned by a solution made up of a $10 \%$ solution of sulphocyanide of ammonium and $1 \%$ solution of chloride of gold. The print is first well washed in several changes of running water until the water ceases to acquire a milky appearance. To 1000 c.c. of water then add 1 c.c. of the sulphocyanide solution, and 
1 c.c. of the gold-chloride solution. The print, after washing, is placed in this gold-toning bath, and toning is allowed to proceed until the stage at which the desired colour occurs has been passed; that is, the process is allowed to go on farther than appears necessary, as some of the acquired colour is lost in fixing. The print is washed in several changes of water, and is then fixed in a solution of hyposulphite of soda -half the strength recommended for the fixation of negatives. The print is again washed for an hour or more in running water, and hung up by a corner to dry. It is important to note that the solution for toning must be used in a perfectly clean dish and measure, so that no contamination from developing-solutions result. The hyposulphite solution must also be quite fresh and not one that has been used for any other fixing purpose.

The artificial-light printing method is that usually referred to as bromide-printing; and possibly from most points of view it is to be preferred. Bromide papers are much more sensitive to light, and must be dealt with in the dark-room as in dealing with sensitive plates, except that their sensitiveness is much less, and the developing process may be carried out in yellow or orange light. A white paper, and one having a highly glazed surface, should be chosen. Such papers are made in varying degrees of rapidity, and may be described as rapid, slow, and very slow. The rapid papers are those usually sold for enlargement purposes, whereas the very slow ones are usually known as 'gaslight' papers. The latter may be dealt with in any weak artificial light other than actual non-actinic light. To obtain the most brilliant results it is, however, better with any of these papers to work in yellow light, as even the gaslight ones are still sensitive enough to become somewhat veiled if exposed to white light for any length of time.

The choice of a rapid or slow paper will depend largely on the negative; and it is possible by selecting a slow bromide paper to obtain a much brighter and harder print from a weak negative than can be obtained on a more rapid paper. This point is one of considerable importance, and where negatives varying considerably in density are being dealt with, the results may be modified within wide limits by selecting a paper suited to any particular class of negative. As the image 
on these papers is produced by development, the result also may be controlled by varying the constituents of the developer, or by stopping the action at an earlier or later stage.

The printing process is carried out by placing a piece of the paper, as before, in contact with the negative in a printing-frame, and then exposing it to a suitable artificial source of light. Almost any light-source may be utilised, except that it is generally not advisable to have it of too high actinic value. An ordinary gas-flame, an incandescent electric lamp, or an oil lamp, may be used at will.

To ensure fairly uniform results, some arrangement should be made for making the exposures at definite distances from the light-source. With ordinary illumination the light-action will vary as the square of the distance; so that if the distance of the light-source from the printing-frame is doubled, the exposure will require to be quadrupled to obtain the same result. In practice, it is found that increasing the distance of the source of light tends to decrease its penetrating power, hence a somewhat harder result is obtained by thus increasing the distance even if the proper proportionate exposure is given. No definite rule can be laid down as to exposure, for it varies with the source of light, and the rapidity of the paper itself. Two or three trial exposures, in conjunction with the instructions given by the makers of the paper, will indicate the time to be given. The results should be recorded so that under the same conditions they may always be repeated.

Development may be effected by almost any developer except pyrogallic acid. Metol-hydrokinone, already mentioned in dealing with negatives, may be utilised, and is in general quite satisfactory. Possibly the best developer for bromide work is amidol, as it gives a very pure black image, and is entirely free from any tendency to stain. A $5 \%$ stock solution of sodium sulphite may be made up, and when it is required for use, one grain of amidol per ounce of solution may be added. Amidol does not keep well in solution, so that enough should only be made up to be used at the time, or at the utmost, within a day or two. The exact proportion of amidol is not of importance, and it is generally sufficient, after having weighed out the required quantity once or twice, to make an approximate 
estimate of the amount by taking it up on the point of a knife or on a small spoon.

Neither does sodium sulphite in solution keep well; so that no more of this should be dissolved than may be required for use in the course of a week or two. No bromide is necessary with this developer, since, given the correct exposure, the results are easily controlled. Several prints may be successively developed in the same solution, but it should not be used after the action is evidently getting slow. When the developer becomes too weak, discoloration or staining may result.

The time taken for development is usually short, but varies somewhat with different makes of paper : as a rule it is complete in from three to four minutes. With some papers, although bromide is not an essential, it is still permissible to use it to restrain the developing action. It should, however, only be used in small quantity, since otherwise it tends to cause deterioration of the black image-a muddy brown-black colour resulting.

Whichever type of printing-paper has been used, it is advisable for the purpose in view to glaze the print, so that the surface is as flat and even as possible. This is best done by allowing the print to dry after its final washing, and then to soak again for about five minutes in cold water. Take a piece of perfectly scratchless plate-glass, or a ferrotype plate, and thoroughly clean it, avoiding hard rubbing or scratching of the surface. Next rub over the surface of the plate a thin film of a solution made by dissolving white wax in benzol or zylol. Polish the plate, leaving just sufficient of the wax on the surface to prevent adhesion of the print. Lay the wet print, face downwards, on the plate and well rub it on the back with clean blotting-paper to ensure that no water remains between it and the print. To do this most effectually an india-rubber rollersqueegee may be used. Place the whole on edge to dry, and if the process has been properly carried out, the print will when thoroughly dry leave the plate of its own accord, or it may be loosened at one corner and then drawn off. The result should be a highly glazed surface, which usually adds considerably to its brilliancy as a print. A still more highly glazed surface may be obtained by coating the print with a thin layer of collodion. 
The plate should be cleaned and polished as before and then coated with what is known as enamel collodion. When the collodion has set, the print, which has been soaked in water, is placed in contact with the collodion surface and squeegeed as before; this produces a still more highly glazed surface. The latter process is, however, rarely necessary, as most P.O.P., or bromide papers made with a glazed surface, can be rendered sufficiently glossy without collodion.

Lantern-slides.-For projection purposes a positive on glass is required, and these are generally referred to as lantern-slides. Their production is quite simple, and is essentially the same as the bromide-printing process on paper. As with ordinary negative-making, dry-plates may be used ; or the wet-collodion process may be adopted. If the negative from which the slide is to be made is of the required size, then that known as the contact-printing method is suitable, when dry-plates are used; but where the negative is larger than the size of the lanternslide, the process known as reduction must be resorted to. Lantern-slides are always made $3 \frac{1}{4}$ inches square; and, allowing for a margin for binding and masking, it follows that no negative in which the part to be used is more than 3 inches square, can be employed for contact purposes. Most makers of plates suitable for negative-making also manufacture those for lantern-slides. Some recently introduced by Messrs. Wratten \& Wainwright are amongst the best of these; the grain of the ones supplied by them is extremely fine, and they are capable of development by almost any process.

The process consists of putting a lantern-plate in a printingframe in contact with the negative to be printed from, so that they are film to film. This must be done in the dark-room by orange or yellow light. The exposure may be made by any artificial light-source, and its length will depend on the negative, source of light, and the sensitiveness of the plate in use. Makers generally give in each box of plates a sufficiently complete set of instructions both as to exposure and development.

In photo-micrographic work the slides are usually required to be bright, and as a rule are preferably black in colour. The metol-hydrokinone developer, already described, gives this 
satisfactorily with any plate; or an amidol developer may be substituted-a suitable one is as follows:-

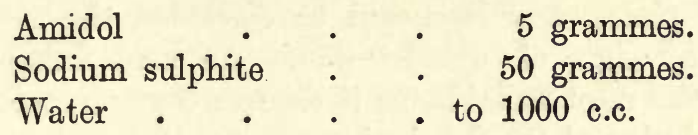

This solution should be made up as required.

Make a stock solution as follows:-

Sodium sulphite 200 grammes.
Potassium metabisulphite. 25 grammes.
Water . $\quad$ to 1000 c.c.

When required for use, take dry amidol and add two to three grains to each ounce of the above.

The first developer will only keep for two to three days at the outside, and a stock solution should therefore be made up and the amidol added dry as described in the second case.

An alternative, and almost universal developer, is as follows :-

Solution 1.

Potassium metabisulphite . 2 grammes.

Ortol . . . . 1 gramme.

Hydroquinone . . . 1 gramme.

Distilled water . $\quad 600$ c.c.

Solution 2.

Potassium carbonate . . 60 grammes.

Distilled water . . . 600 c.c.

For use, take equal parts of solutions Nos. 1 and 2.

Development is carried out in exactly the same manner as negative-making; but the process must be carried as a rule only far enough to give a bright-looking positive. No part must be so dense that when it is projected on to the sereen it will not allow light from the projection apparatus to penetrate. Some little experience is necessary before a good lantern-slide can be produced; but once this experience is acquired, there is no particular difficulty. In any case, a good slide should have at least some small part in which there is, on fixing, absolutely clear glass. Development is usually much more rapid than when making a negative, and the beginner will probably err on the side of over-development. 
Fixing is effected in exactly the same way as with the negative, except that fresh solution should be used for each batch of plates, and it should be discarded the moment it shows any sign of discoloration. An acid fixing-bath is perhaps the most suitable, as it ensures that no stain results from oxidation of the developing agent. It may be made by adding about 20 grammes of metabisulphite of soda to each 500 c.c. of fixing-solution.

To obtain a lantern-slide from a plate which is too large to print by contact, recourse must be had to the reduction process. The method consists essentially of re-photographing the negative by means of an ordinary camera. The latter is placed in such a position in relation to the negative that a reduced image of it is thrown on to the ground-glass screen. The negative is placed in front of the camera, and the latter extended for such a distance that an image of suitable size is seen. The negative may be illuminated by either daylight or artificial light. The image is focussed as in ordinary photography, and the lantern-plate put in the dark-slide and the exposure made. There are many appliances to be obtained commercially for this purpose. They may be had of fixed focus, in which case they are only suitable for reduction from one size of negative, or they may be variable for any size. The important points to bear in mind are that the negative is evenly illuminated, and that the lens used is such that it does not impair the definition of the final result.

Exposure depends on the intensity of the light used; in the case of daylight, from one to three minutes will usually suffice. Development is carried out exactly as when making a slide by contact. This method is rarely necessary in photo-micrography, so that reference should be made to one of the manuals on pure photography if further instructions are required.

The positive having been fixed, washed, and dried, as in negative work, it requires covering with a mask of black paper to cut off any unsightly part of the edge of the image. It is then placed in contact with another plain piece of glass of the same size, so that the film is between the two. Lastly, the edges are bound round with adhesive black paper, and the slide is ready for projection. Lantern-slide making presents no particular difficulty over any other photographic process except 
that absolute cleanliness is perhaps even more essential : any small speck of dust or any imperfection in the negative becomes so painfully evident when a slide is projected on the screen, that it is of the utmost importance to ensure that no such blemish shall be present. Probably all dry-plate lantern-slides can be improved by a short immersion in the Howard Farmer reducingsolution, already mentioned. This is not with a view to general reduction of density, but to brighten up the image and to remove any slight general fog that may be present. It is also at times most useful to apply the solutions by means of a tuft of cotton-wool. By this means local reduction may, if necessary, be effected, and it is sometimes of the greatest value in enabling a good slide to be produced from an indifferent negative.

The most perfect method of making lantern-slides is the wet-collodion process, and, as this process lends itself to negative-making, it will be briefly described.

As already stated, the grain of the image in a collodion process is finer than in a dry-plate; consequently, for lanternslide work, where fine detail has to be shown, or for the highest class of negative-making, it is still unrivalled. For a busy worker there are considerable objections to it: the plate must be prepared and coated before an exposure can be made, and this entails a considerable amount of additional work as compared with the dry-plate process. Lantern-slides thus made are, however, particularly transparent and brilliant; and the process has the added advantage that where a slide has been made from a thin negative and lacks in brilliancy it can be intensified to an extent that is impossible with a dry-plate. The necessary operations are briefly as follows. An ordinary 31 -inch-square glass plate is thoroughly cleansed and coated with collodion. The cleansing process must be carried out very efficiently, and, if the glass has not been previously used, it must be well washed in hydrochloric acid and water, followed by a thorough rinsing in clean water. Before the plate is coated with the collodion, it must be edged to prevent the film slipping off in the subsequent developing operations. This is done by running round the outer edge of the plate, by means of a camel-hair brush, a narrow edging, about one-sixteenth inch wide, of a one-per-cent. solution of india-rubber in benzol, and this must be allowed to dry 
before the plate is coated. A number of plates may be so prepared and kept ready for use. The collodion may be purchased ready iodised, and it is better to have some that has been kept for about a month before it is taken into use; in any case it should have been kept long enough to have changed to a sherry colour. The coating of the plate is not difficult when once the knack has been acquired. Take one of the clean and rubberedged glasses, hold it by the thumb and finger at one corner, and pour on it sufficient collodion to cover about one-third of the plate. Gently incline it to each corner in succession, so that the collodion is spread evenly over the surface. When the last corner has been covered, tilt the plate and pour any surplus collodion back into the bottle. While the plate is draining, it should be rocked backwards and forwards in order to avoid the formation of ridges as the film sets. The coating operation can be performed in daylight, since up to this point it is not sensitive to light; but the next, the sensitizing process, must be carried out in yellow or orange light in the dark-room. The plate is now immersed in a silver-bath, made by dissolving thirty grains of nitrate of silver in one ounce of distilled water. The solution should be distinctly acid to litmus, and if it is not so, must be made acid by the addition of nitric acid drop by drop. The solution must be filtered before use. After coating the plate with the collodion film it must be allowed to set for from one to three minutes, according to the temperature, but not sufficiently long for it to become dry in any part. The nitrate of silver solution should be placed in a porcelain dipping-bath, such as may be obtained from any dealer. The collodionised plate having just set is immersed in the solution, and allowed to rest there for about a minute. The plate should next be raised and, if necessary, re-immersed until all appearance of greasiness disappears. The plate may be allowed to remain about a minute after this appearance has been reached, and it will then be ready for exposure. The time it takes to sensitize will usually vary from three to five minutes. The plate is put into a dark-slide, which should have silver-wire corners fitted to it to avoid contact with the film more than is absolutely necessary, and the exposure made.

As in dry-plate work, no definite instructions can be given as to the length of time necessary for the exposure, for this 
will depend upon the light and density of the negative. But, in general, it may be taken that it will be approximately twice as long as for a dry lantern-plate of average speed. A full exposure is advisable, as intensification of an over-exposed plate is quite practicable.

Exposure having been effected, the plate is removed from the slide in the dark-room, and development carried out. A suitable developing-solution is as follows:-

Ferrous sulphate

Glacial acetic acid

Alcohol

Water
1 gramme.

0.5 c.c.

2 c.c.

100 c.c.

The solution should be kept for a few days before use. It tends to improve with age, but should be discarded when turbidity becomes evident.

About 10 c.c. of this solution is poured on to the centre of the plate, the latter being held between the thumb and finger, and the developer caused to flow from corner to corner. It is important that it should flow over the plate as quickly and evenly as possible, since the image comes up almost at once. Sufficient solution should be used to secure this, but not enough to cause any of the developer to flow over the sides. The plate should be slowly rocked from side to side until development is complete, when it should be gently rinsed under the tap and fixed in a solution of hyposulphite of soda of the usual strength. The image will appear much sooner than in a gelatin plate, and will more quickly gain the required density - the whole operation being completed in from one to two minutes. It is important to see that the developer is not allowed to flow off the plate, as the action depends to a considerable extent upon the free nitrate of silver which remains in the film helping to bring up the image. The loss of density in fixing is slight. Exposure and development should be carried through as quickly as possible after the preparation of the plate to avoid partial and uneven drying.

The advantage of the wet-plate now becomes evident. It is possible to intensify a thin slide, or one from a flat negative, and to make it almost, if not quite, as good as a slide from 
a technically good negative. In gelatin plates this is also possible up to a certain point, but the slide is likely to suffer much more from stain and loss of gradation than in wet collodion. Intensification is effected by applying to the slide, after fixation and washing, the following solution :-

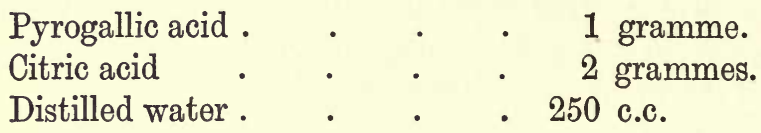

Just before applying this solution to the plate, a few drops of a five-per-cent. solution of silver nitrate in distilled water may be added; but this should only be done immediately before use, and to such a quantity of the solution as will be used at once. The solution is poured over the plate exactly in the same manner as in development, and the treatment is continued until sufficient density is obtained. Should the solution become turbid before completion, then it must be washed off and a fresh portion applied. The plate is finally washed under a gentle stream of water, and dried.

In pictorial photography it is usual to tone collodion slides, as the colour is often not all that might be desired. For the purpose under discussion, however, there would not generally appear to be any necessity to go to this additional trouble, since, providing the slide is of a suitable black colour, such after-treatment is unnecessary.

In case the slide may not have acquired such a black colour by development, it may be altered by toning in a solution of chloride of gold in distilled water. The strength may conveniently be one grain of gold chloride to ten ounces of water. As the film of a collodion slide is much more liable to injury than gelatin, it is advisable to varnish it. Any clear colourless negative-varnish may be used.

In the formulæ given in this section, the decimal system has as far as possible been adhered to ; but in one or two cases, as a matter of convenience, this has been departed from. For instance, it is universally the custom to take one ounce of developing-solution as the normal quantity for a quarter-plate; a departure in such a case, therefore, would not appear to be without justification.

A few words may be useful as to the method of projecting 
lantern-slides. The essentials are a good source of light, and this should preferably be one of small area-such as oxyhydrogen or electric arc-a projection-lens which is aplanatic, and a screen which has a perfectly dead-white surface, and is opaque. The latter point is perhaps in some respects the most important, and it is certainly the one to which least attention is usually given. A small, brightly illuminated picture is much to be preferred to a large, badly lighted one, and it therefore follows that, except for demonstrations to large audiences, a picture from six to eight feet in diameter will show in any ordinary lecture-theatre much better than one of larger size. 


\section{CHAPTER XI}

\section{SOME SPECIAL PROCESSES}

Photo-micrography by means of Ultra-violet Light.-It has already been set out that the resolving-power of objectives increases directly in proportion to any decrease in the wavelength of the light used. To take full advantage of this, Messrs. Zeiss have recently produced an apparatus, designed by Dr. Kohler, in which the source of light lies in the region of the ultra-violet; and the wave-length of that light is such that the resolving-power of the optical system is double that of any objective used for visual work.

It is well known that many organic substances are opaque, or partially opaque, to ultra-violet light, although under ordinary conditions of illumination no difference in transparency of the component parts is to be discerned. It therefore follows that by using such ultra-violet light for illuminating purposes structure may be brought out, which by any other method of illumination, or by any attempt at differential staining, is invisible; the effect being that although an object may appear colourless by white light, it would in fact appear to have colour differences if illuminated by ultra-violet light. It is obvious that no direct observation of the image can in this case take place, and that photography is the only means by which a record of the structure of such objects can be obtained.

The only method by which visible effects may be observed is when the ultra-violet light exerts a very definite physiological action upon living or similar objects which are under the microscope. It is well known that uitra-violet light has a very marked bactericidal effect, and that its physiological effect on various living organisms and tissues is often well marked. 
It is therefore possible to allow the light to act on such organisms or tissues, and to observe what happens with an ordinary microscopic objective corrected only for white light. In this case the ultra-violet light plays no part in the formation of the image, but is only instrumental in producing the effect which is to be observed.

The objectives made for direct use with ultra-violet light are called 'monochromats,' and the wave-length for which these are corrected is $275 \mu \mu$. The N.A. of the highestpower lens of this series is $1 \cdot 25$; but by virtue of the shortness of the wave-length of the light, the resolving-power is equal to an objective used with white light of 2.5 N.A.-an objective which of course at the present time it has not been possible to produce.

The lenses of these monochromatic objectives are made exclusively of fused quartz, so that they are transparent to the whole range of the ultra-violet spectrum; that is, they transmit all radiations until the point is reached where light is of such short wave-length that it is absorbed by the atmosphere.

The two highest-power objectives are immersion-lenses, the immersion-fluid being a mixture of pure glycerin and distilled water used in such proportions as to be of the required refractive index. The cover-glasses and the slides also consist of fused quartz; or, alternatively, the slides must be made of uviol glass, which is sufficiently transparent to ultra-violet for most purposes.

A series of oculars, the lenses of which are also made of quartz, serve to project the image on to the photographic plate, and the numbers by which these oculars are known indicate their magnifying power, exactly in the same manner as in the case of compensating oculars.

It is convenient to use a photographic apparatus of the vertical type; in fact, the Zeiss vertical camera is the one usually recommended for this purpose, and is made in a suitable form with certain modifications from the ordinary type.

For direct observation - that is, for finding the object, centreing the image, and for preliminary observation-a searcher eye-piece is employed. It is so arranged above the microscope that it can be used for observation and easily swung out of position when the use of the camera is required ; either appliance 
may therefore be used alternatively. In the searcher eye-piece is a small fluorescent screen, and a high-power magnifier is arranged so that the image formed on this screen may be directly observed. After the image has been adjusted exactly on the fluorescent screen, it will then be in focus when the camera is placed in position and the photographic plate substituted. Variations in the length of the camera, not exceeding a few centimetres, will not have any considerable effect upon the sharpness of the image.

The source of light is supplied by passing a high-tension discharge from a coil and a Leyden jar between cadmium electrodes. The actual production of the necessary high-tension current may be by means of an induction coil giving a spark, say, about three inches in length. The light emitted by the electrodes is split up, by special illuminating apparatus consisting of lenses and prisms of quartz; the light of $275 \mu \mu$ which is emitted from the cadmium electrodes, or of $280 \mu \mu$ which may be used alternatively from magnesium electrodes, is spread out into a spectrum, and the portion not required is cut off by means of an iris-diaphragm. This diaphragm allows a beam to pass which is collected by a condenser of quartz taking the place of the ordinary sub-stage condenser of the microscope; the rays are thus focussed with a narrow or wide cone, as required, on to the object and pass into the objective.

Table $\mathrm{V}$ gives the range of magnification that may be obtained, and it will be seen that there is almost as much range as would be obtained with a battery of ordinary objectives. The optical camera-lengths are given in centimetres, as with ordinary objectives. To obtain exactly a given magnification, it is advisable to use a stage-micrometer, altering the cameralength until the exact magnification required is obtained.

It will be appreciated that in addition to the increased resolving-power, this method is in effect another means of differential staining. All bodies to be photographed by this means must be unstained, and any detail of structure will be brought out as the result of differences in the absorptive-power of the tissue to ultra-violet light. The preparations must not be fixed, dried, or otherwise treated in the way that microscopical preparations are usually dealt with. It is also important to remember that the medium in which the preparation 


\section{Table V}

\section{MAGNIFICATIONS AND OPTICAL CAMERA-LENGTHS}

For the Monochromats and the Quartz Eye-pieces at $160 \mathrm{~mm}$. Tube-length and $\lambda=275 \mu \mu$.

\begin{tabular}{|c|c|c|c|c|c|c|}
\hline Objectives. & Eye-pieces. & 5 & 7 & 10 & 14 & 20 \\
\hline \multirow{4}{*}{$\begin{array}{c}6 \mathrm{~mm} . \\
\text { N.A. } \\
0.35\end{array}$} & Magnifications & 200 & 300 & 450 & 600 & 900 \\
\hline & $\begin{array}{c}\text { Optical } \\
\text { camera-lengths }\end{array}$ & $24 \mathrm{~cm}$. & $25.5 \mathrm{~cm}$. & $27 \mathrm{~cm}$. & $25.5 \mathrm{~cm}$. & $27 \mathrm{~cm}$. \\
\hline & Magnifications & 250 & 400 & 500 & 800 & 1000 \\
\hline & $\begin{array}{c}\text { Optical } \\
\text { camera-lengths }\end{array}$ & $30 \mathrm{~cm}$. & $34 \mathrm{~cm}$. & $: 30 \mathrm{~cm}$. & $34 \mathrm{~cm}$. & $30 \mathrm{~cm}$. \\
\hline \multirow{3}{*}{$\begin{array}{c}2.5 \mathrm{~mm} . \\
\text { N.A. }\end{array}$} & Magnifications & 500 & 700 & 1000 & 1400 & 2000 \\
\hline & $\begin{array}{c}\text { Optical } \\
\text { camera-lengths }\end{array}$ & $26.5 \mathrm{~cm}$. & $26.5 \mathrm{~cm}$. & $26.5 \mathrm{~cm}$. & $26.5 \mathrm{~cm}$ & $26.5 \mathrm{~cm}$. \\
\hline & Magnifications & 600 & 800 & 1200 & 1600 & 2400 \\
\hline 0.85 & $\begin{array}{c}\text { Optical } \\
\text { camera-lengths }\end{array}$ & $31.5 \mathrm{~cm}$. & $30 \mathrm{~cm}$. & $31.5 \mathrm{~cm}$ & $30 \mathrm{~cm}$. & $31.5 \mathrm{~cm}$. \\
\hline \multirow{3}{*}{$\begin{array}{c}1.7 \mathrm{~mm} . \\
\text { N.A. }\end{array}$} & Magnifications & 700 & 1000 & 1500 & 2000 & 3000 \\
\hline & $\begin{array}{c}\text { Optical } \\
\text { camera-lengths }\end{array}$ & $24 \mathrm{~cm}$. & $24.5 \mathrm{~cm}$. & $26 \mathrm{~cm}$. & $24.5 \mathrm{~cm}$ & $26 \mathrm{~cm}$. \\
\hline & Magnifications & 900 & 1300 & 1800 & 2500 . & 3600 \\
\hline 1.25 & $\begin{array}{c}\text { Optical } \\
\text { camera-lengths }\end{array}$ & $31 \mathrm{~cm}$. & $32 \mathrm{~cm}$. & $31 \mathrm{~cm}$. & $31 \mathrm{~cm}$. & $31 \mathrm{~cm}$. \\
\hline
\end{tabular}

The effective N.A. is doubled in each case owing to the wave-length of the light used.

is immersed must be one that does not offer any obstruction to the passage of ultra-violet light. The whole process is not a simple one, and it must be admitted that at first the results may not prove so satisfactory as might be anticipated. It is essentially one that calls for the exercise of considerable patience and not a little skill. But once the manipulation has been 
mastered it is possible to produce photographs of living tissues and organisms, or in an unaltered condition immediately after death, which cannot be accomplished in any other way.

It is in some respects unfortunate that the apparatus is so expensive, as it of necessity limits its application, and except only in scientific institutions few will be able to take advantage of it. The further difficulty of obtaining fresh preparations and having them mounted between the necessary quartz slip and cover-glass presents an obstacle in many cases. Preparations have to be prepared and dealt with immediately, there being little possibility of leaving them over for any lengthened period.

In actual work the length of the spark between the cadmium or magnesium electrodes should be kept as short as possible; what is usually referred to as a 'fat' spark will be the most satisfactory. It is permissible to use an induction coil giving a larger spark than that mentioned; but if so, the spark-gap must be short, so that the spark itself becomes broader. The spark spectrum of the electrodes projected into the apparatus will then have greater intensity. The disintegration of the electrodes takes place, of course, at their surface, so that the shorter the spark-gap, having due regard to the necessity of having an illuminant of sufficiently large area to fill the condenser, the better the result.

The greatest practical difficulty is in focussing the objective and the sub-stage condenser, whether dry or immersion. In either case, the image as seen with a searcher eye-piece may not give the best result in the photograph. As in photographing spectra, the best result is not always obtained when the best visual image is seen on the focussing-screen; hence it is largely a question of trial and error. Alternatively, the searcher eye-piece may be dispensed with and a series of exposures made, with the fine-adjustment of the microscope so arranged that successive planes at short distances apart are brought into focus. A graduated milled head on the fine-adjustment is in this case essential, and a succession of photographs would be taken, the milled head being moved backwards or forwards by one graduation only in each case. If this method is adopted it is advisable to get an approximate image with the searcher eye-piece and then to begin the series of trial exposures. The 
method is in practice somewhat laborious; but probably the results obtained, at least with suitable preparations, will ultimately prove to be the best.

The amount of contraction permissible with the sub-stage iris-diaphragm must be determined by experiment. As in visual work, this must not be carried to excess ; but possibly, since contrast is only secured as the result of differences in lightabsorption, a rather narrower cone may be used than when using ordinary objectives with white light.

Another method of getting a sharp image is to do exactly what was formerly done when using achromatic objectives not corrected for photography. This is to determine the distance between the actinic and the visual focus, and to make definite allowance by means of the fine-adjustment before making the exposure. In the case of the ultra-violet apparatus, an image may be focussed with white light, and then a succession of photographs taken with the ultra-violet apparatus, the fineadjustment of the microscope being moved a definite amount each time. On developing, the image that gives the best and sharpest result will be an indication of the amount that the fine-adjustment must always be moved if the object is first illuminated by white light and then the photograph taken by means of the ultra-violet. A quartz micrometer-slide may be obtained from Messrs. Zeiss, by means of which these determinations may conveniently be made.

As the process deals entirely with light of highly actinic value, the particular plate used is not of great importance; except that, to ensure fineness of grain in the resulting photograph, a slow one is preferable. No advantage is to be gained by using orthochromatic plates; in fact, any slow process or lantern-plate will give a good result. It is also not necessary to use a backed plate; the light will not pass through the glass and is in great part stopped by a gelatin film, so that backing a plate becomes superfluous.

As already stated, the preparations must be specially prepared. Canada balsam and zylol are both opaque to ultraviolet, and sections which have been passed through zylol must have the latter completely removed. They may be finally mounted in glycerin or in agar-salt solution.

The method of photographing by ultra-violet light is 
essentially one that should not be attempted by a novice, but only by those who have already had a fairly wide experience in other branches of the work. It presents all the usual difficulties of dealing with transparent preparations, with the additional one that in no case can the direct image be dealt with; in fact, it is only when the photograph is actually developed that a definite idea as to whether success has been attained or not can be arrived at.

The Photography of Metal Surfaces.-To obtain photographs of such objects certain special conditions have to be complied with. The main point of difference between these and transparent objects is in the method of illumination. In the section dealing with the various appliances for efficiently illuminating objects, some description has already been given of what are known as opaque illuminators, and it is in the correct use of these appliances, and so obtaining proper illumination of the object, that the chief difficulty exists. It is also necessary to have in the microscope itself certain modifications of construction to enable the object and the additional optical appliances to be dealt with more easily.

A further difficulty lies in the method of focussing. It is obviously a great disadvantage, where an ordinary stand is employed, if the position of the object cannot be altered without disturbance to some of the illuminating arrangements. Fig. 70 shows the general arrangement when a metal surface is illuminated by means of the vertical illuminator. From this it will be seen that the illuminant $\mathrm{A}$ is a fixed point, and that the position of the reflecting-mirror $B$ at the back of the objective should be constant. To enable this to be done it is therefore advisable for the stage of the microscope itself to have a coarse adjustment, and that an accurately made one, so that the object may be brought up to its position in correct focus, leaving only the final exact focussing to the fine-adjustment, if such is necessary at all.

A typical instrument of this class is the Zeiss large photomicrographic stand arranged for either transmitted or reflected light. This instrument is the usual standard type for photomicrographic work already figured and described; but it has in addition a coarse-adjustment, which moves the whole of the stage and sub-stage apparatus. It also has attached to one 
side of the body-tube a mirror which has universal motion and is adjustable in the direction of the length of the tube, so that for low-power work with metals illumination may be carried out by obliquely reflected light. The mirror is in the latter

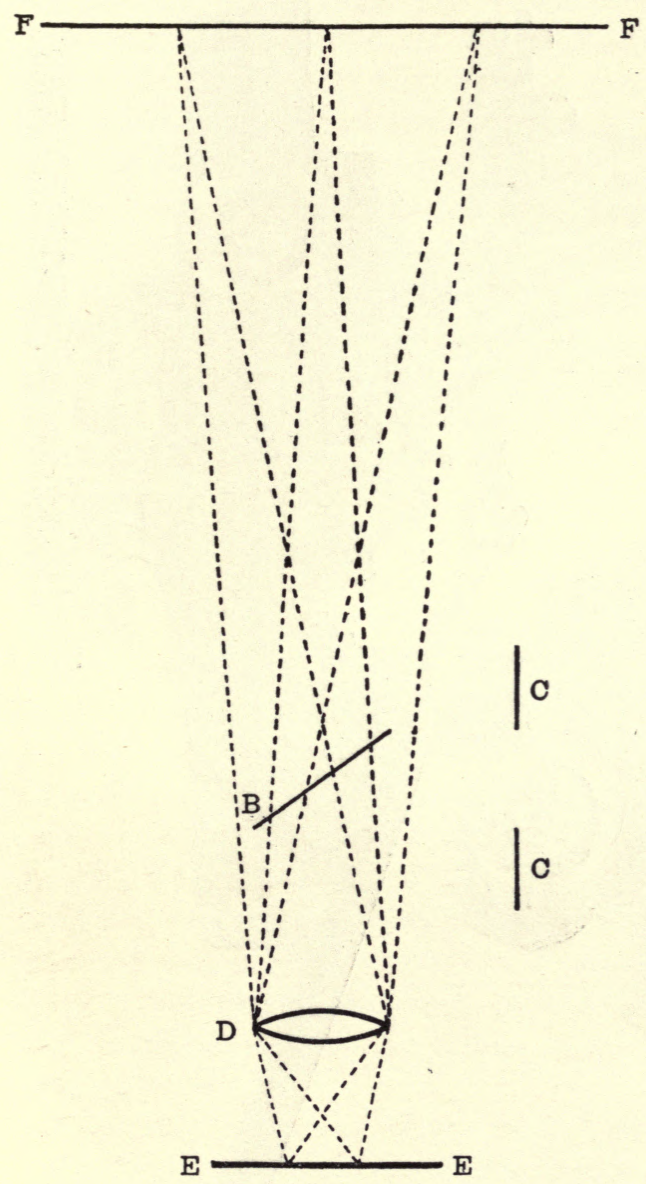

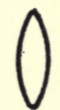

Fic. 70.-Diagram of Path of Rays in Vertical Illuminator.

case first adjusted approximately in position in relation to the surface of the object, and then a parallel or convergent beam is projected on it and reflected on to the metal surface. This instrument is unfortunately not so stable in the horizontal position as could be wished, but in every other respect it 
exactly fulfils the requirements for the most advanced and critical work. The body-tube is of large size, as in the ordinary photo-micrographic stand, to minimise internal reflection and

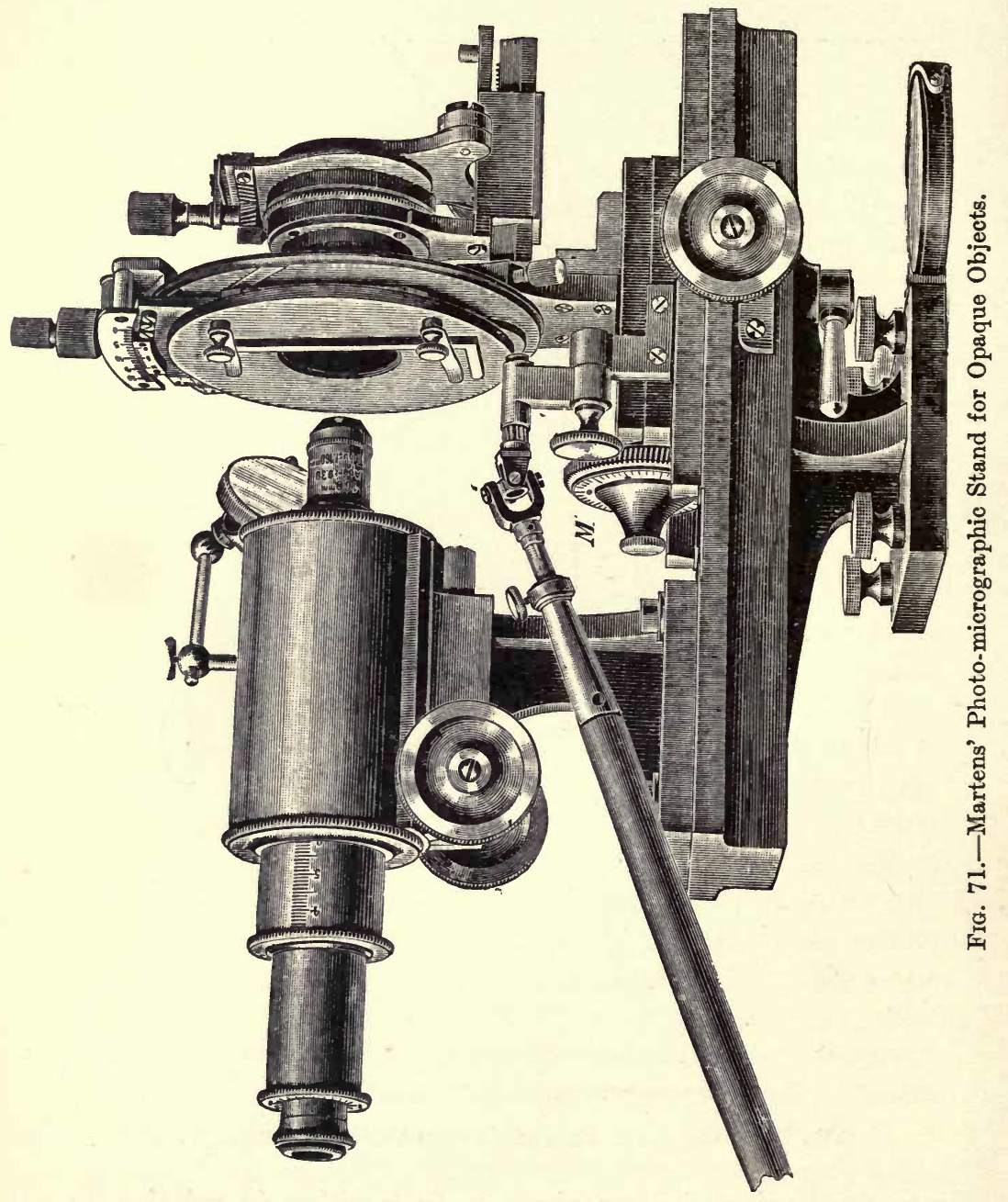

to enable Planar and similar long-focus lenses to be used. The stage has mechanical motion, and may be rotated. In metal-work the latter is a point of great importance, as often the direction of incidence of the light in relation to the 
specimen requires to be varied to bring out some particular structure.

To overcome the want of stability in the horizontal position Messrs. Zeiss have recently constructed a stand.which is known as Martens' stand (Fig. 71). This instrument may be used only when illumination by reflected light is required, and it differs in design from any other at present in use. The fineadjustment motion $M$ controls movement of the stage and not of the body-tube at all, so that in using the vertical illuminator there is no alteration whatever in the incidence of the light. Some modifications can be introduced to enable it to be used for transparent objects; but owing to the fact that it is only available in the horizontal position it is not so convenient for purposes other than photo-micrography or direct projection. It must be admitted that as a stand for photo-micrographic work, pure and simple-even excluding its applicability for metal-work-it is almost ideal, as the position of maximum ability is attained when horizontal; and, owing to the fact that all the adjustable parts are supported in long slides, it is very difficult for any flexure to take place even during a prolonged exposure. For very high-power work of the most critical nature, it is exceptionally stiff and rigid.

The general arrangements for metal-work are the same as in the previously described stand. The method of controlling the fine-adjustment is a direct one. It is connected by a Hooke's joint to a long arm, so that no intermediate cords or pulleys or any gear-wheel arrangement for connecting up the fine-adjustment is necessary. Rotation of the stage and mechanical motion are both provided for, and verniers are. also supplied, so that any precise position of the stage may be arranged for.

Another metallurgical microscope of special design is that made to the specification of Mr. Walter Rosenhain by Messrs. R. \& J. Beck (Fig. 72). This instrument is one of great interest, as in the working out of the model no regard has been paid to any previous designs of stands. The point to which great attention has been paid is stability; in this respect the instrument has certainly no superior, and, for one that allows of use in the vertical or horizontal position, has probably no equal. The limb has been designed on engineering principles, 
so that it really constitutes a properly proportioned truss, being in fact similar in design to a strutted girder. It is made

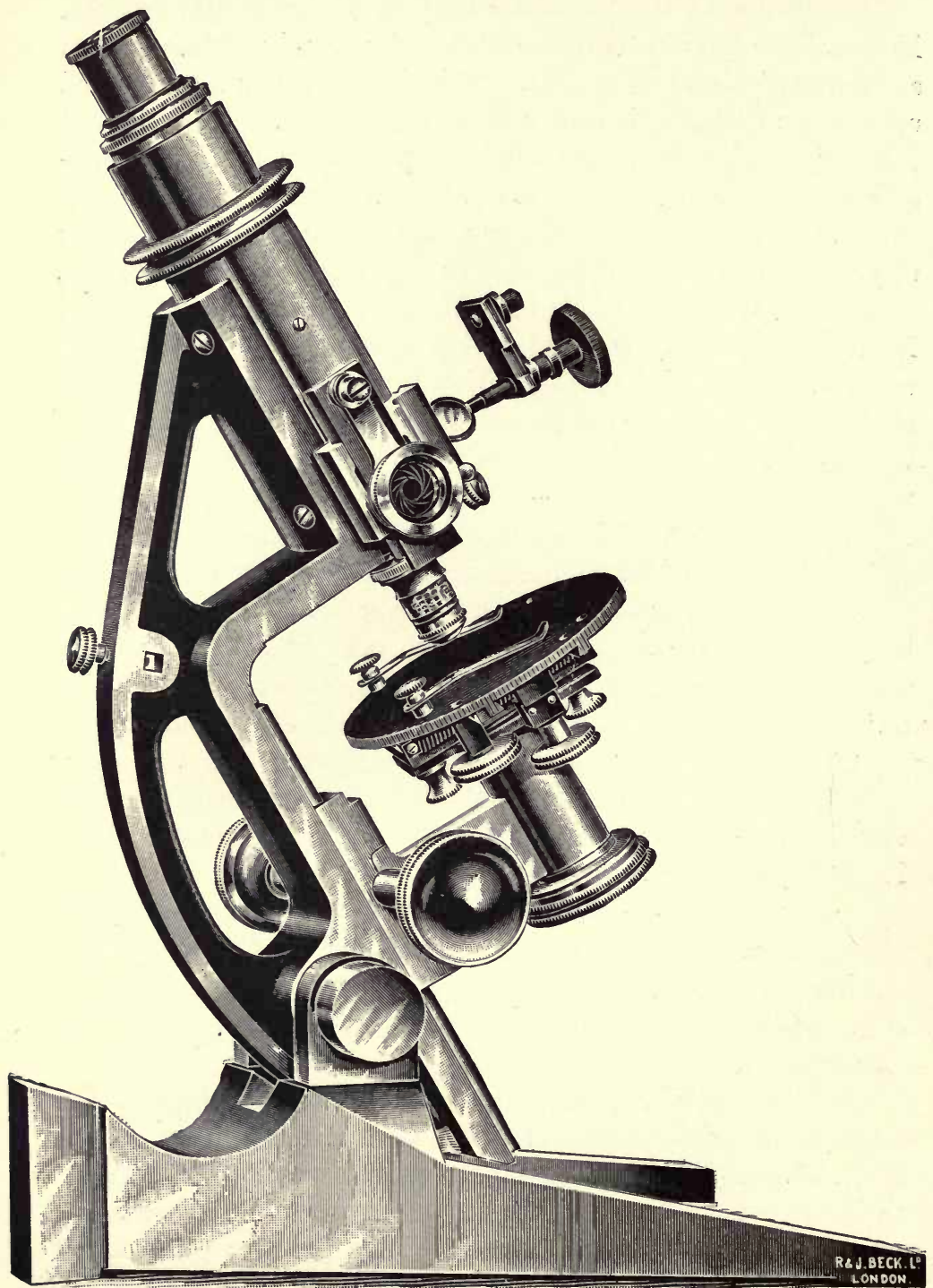

FIG. 72.-Rosenhain's Metallurgical Microscope. (R. \& J. Beck.) in girder-section throughout; the upper portion in front having the body rigidly attached, and the lower front portion having. 
a wide dovetail-fitting upon which the stage racks up and down. The limb is jointed on a centre so arranged that the instrument is almost perfectly balanced when either vertical or inclined at any angle. In the horizontal position it rests on a cradle which forms part of the supporting-base, so that it is then perfectly rigid. The design is such that any tendency to vibrate is much reduced; in fact, any vibration transmitted to the instrument would have the minimum effect in disturbing any of its parts. The base has a very wide spread, so that in this particular also great stability is obtained. The coarseadjustment has a range of $3 \frac{3}{4}$ inches when the instrument is vertical, and a further 1 inch when the instrument is at a slight angle or horizontal. The fine-focussing is situated in the optio axis immediately under the object. The stage is carried on a triangular stem in a long triangular fitting in the stagesupport, and owing to the large surface of contact over all these fitting parts, is also very rigid. The body-tube is made of extra thickness; and at the lower end is a nosepiece for carrying the objective, with centreing-adjustments. It follows that different objectives can be exactly adjusted to the optic axis of the microscope, ensuring that on rotation of the stage the object remains central. Three fittings are supplied at the lower end of the tube, into which all illuminating appliances and their diaphragms may be attached to the instrument. These include any type of vertical illuminator for throwing direct light on to the object : the parabolic or Sorby illuminator, already described, or a set of coloured-glass screens, suggested by Mr. Rosenhain, for simultaneously illuminating with light of different colours in different directions. A supplementary draw-tube is provided for lengthening the body-tube when this is required, and to allow of the use of very low-power microscope objectives or photographic lenses. The stage admits of complete rotation, and may be clamped in any required position, allowing illumination to be effected at any angle. The centre of rotation of the stage is as exactly as possible at the optic axis of the instrument, and, as already mentioned, centration is effected by adjusting the position of the objective and not by altering the position of the stage itself. Mechanical motion of the stage is provided in both directions. The fittings for carrying the vertical illuminator are so arranged 
that the illuminator may be used in any direction-that is, in front or at either side. By sliding it up and down in a dovetailfitting the illuminator can be placed at any given distance from the back lens of the objective, and may also be moved at right

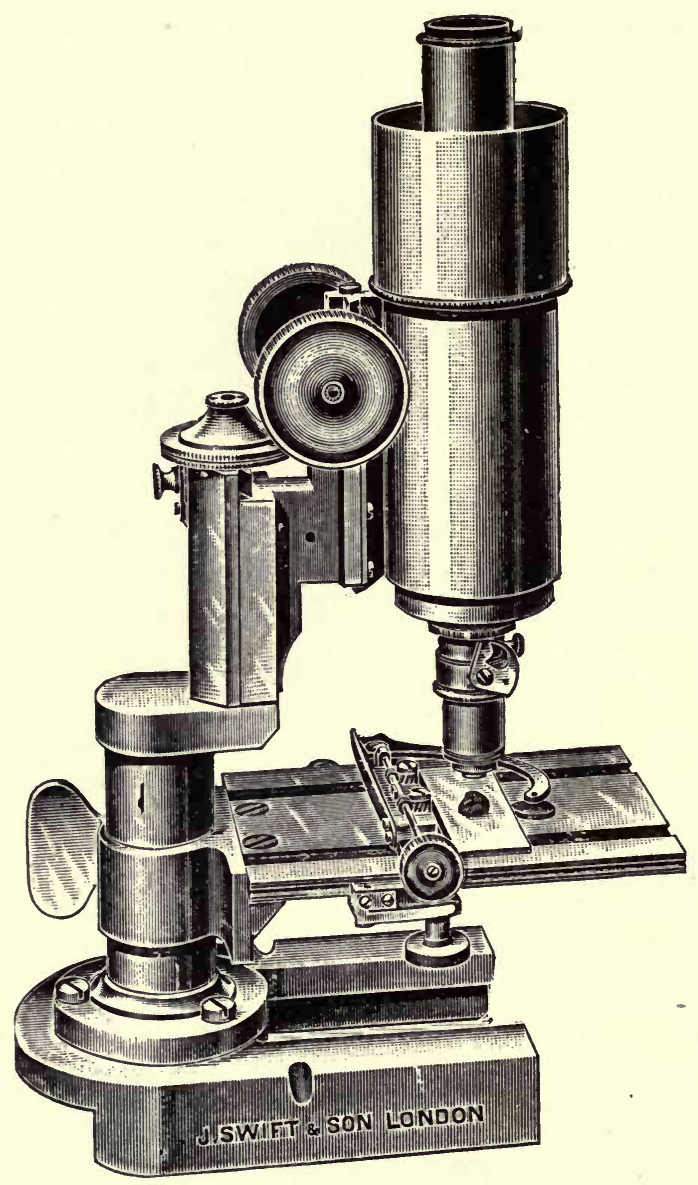

Fig. 73.-Engineers' Metallurgical Microscope. (J. Swift \& Son.)

angles to the optic axis. An iris-diaphragm is also fitted for use in conjunction with the vertical illuminator, and this constitutes a most valuable accessory in actual work, as it is often necessary to be able to reduce the angle of the illuminating beam. 
Another instrument of special design is that suggested by Mr. J. E. Stead, F.R.S., and made by Messrs. J. Swift \& Son (Fig. 73). This one differs again, as it is only available for use in a vertical direction; but it is made very stiff, so that in cases where a photograph of a fractured surface of a large forging, or similar large mass of metal, has to be obtained in situ, the microscope may be clamped direct on to the specimen. It is of massive construction, and has a solid stage made to swing out of position, so that the objective may be brought into focus on any large object upon which the foot is supported. To effect this an inside-tube carrying an object-glass slides within the outer barrel, and can be lowered to a sufficient distance. For photo-micrographic work a small conical camera is placed on top of this barrel, and a photograph may then be taken directly. This instrument is hardly intended for use with high powers or for work of a critical nature; but it fulfils the purpose for which it was designed with very great efficiency, and it enables objects to be dealt with that under any ordinary circumstances could not be examined with a microscope at all.

It has been necessary to give a detailed account of these instruments, as they differ in important respects from the ordinary microscope, as already pointed out; and for this reason, therefore, they were not dealt with in the chapter devoted to microscope design and construction. As to the objectives for metal-work, they differ only in one particular, and that is that-except in the case of oil-immersion lenses-they must be corrected to work correctly on objects that are not mounted beneath a cover-glass. Metals usually have polished or etched surfaces, and when vertical illuminators are used it is not admissible to interpose a cover-glass between the objective and the object. It is, therefore, absolutely necessary to ensure that all dry lenses are specially corrected, and it is not possible to use them at their best, except with very low powers, unless this is the case.

Illumination must be effected on the same general principles as in work by transmitted light ; that is, with low-power work a beam must be projected on to the specimen so that the field is evenly illuminated, and in high-power work a critical image of the illuminant must be exactly projected on the surface of the object. This is in general a matter of no difficulty. 
The illuminant is placed at a distance of about nine or ten inches from the body-tube, and it will then generally be found that the image of the illuminant is practically in focus when the surface of the object itself is in focus. Any alteration in this respect to ensure a more exact focussing of the illuminant must be effected by altering the distance of the illuminant from the microscope itself. Where a bull's-eye or other condenser is interposed between the source of light and the microscope, it is advisable to use a parallel or only slightly convergent beam. The size of this beam should be cut down by an irisdiaphragm CC (Fig. 70), so that no stray light is allowed to enter the objective. The exact cutting down of the dimensions of the beam is, perhaps, one of the most essential points to attend to in metal-work, as it is obvious that with the highly reflecting surfaces of metals, considerable diffusion of light may easily occur. In the later forms of vertical illuminators this is provided for so that not only the beam impinging on the illuminator, but the actual size of the beam entering the back combination of the objective may be regulated at will.

The range of magnification required is not so great as in work with ordinary transparent objects; in fact, observation of published results shows that a great deal of it is done at magnifications of either 100 or 1000 diameters. In the early days of metallography it was thought that only low powers were of use in this class of study, investigation by higher powers having failed at that time to lead to any interesting results: It was only when Mr. J. E. Stead took up the subject of highpower work that it was found that a new order of structure could be revealed. It has therefore become almost customary either to treat the object from a low-power standpoint or at once to go to much higher magnifications. The result is that a large range of objectives will not in general be required. Achromatic or apochromatic objectives are available, and the same general conditions apply as in ordinary work. The necessary working N.A. should be as large as possible, since the loss of light is very much greater than in work with transparent objects, with the consequence that unless a high-power illuminant is used the exposures become unduly lengthened.

The time of exposure is not so difficult to estimate as 
when dealing with transparent objects; in fact, so much so is this the case that if an object is, for instance, photographed at 100 diameters with an 8-mm. objective and is then photographed at 1000 diameters with a 3 -mm. oil-immersion, using a vertical illuminator in each case, the exposure, assuming that all other conditions are the same, will be almost exactly the same length of time in each case. The reason for this is that the objective itself being the condenser, its working N.A. increases exactly as its own N.A. increases; the result being that with the high-power objective the image of the source of light is formed by a much wider beam and is of very much smaller dimensions when projected on to the object. It follows that the necessary exposure in each case would be almost exactly the same, except that there is some slight difference in the light-absorbing power of the objective itself which must be allowed for. In actual practice it is found that even with widely varying magnifications, when using the same class of objective, the exposures differ but little.

It does not come within the scope of this work to describe the methods of dealing with metal surfaces to make them suitable for microscopic observation. In general, however, it may be stated that first the object requires to be ground or cut so that it has a plain surface. This surface, then, has to be polished so that any scratches or abrasions are obliterated. The polished surface then requires either etching by suitable solvents, or heating, and cooling quickly or slowly, so that some differentiation, in cases where an alloy is being observed, can be seen between the constituents. For further information on this subject the reader is referred to the many books which now deal with the micro-structure of metals, particularly the works on 'Alloys,' by Mr. E. F. Law, and 'Metallography,' by Dr. C. H. Desch.

The object having been prepared, the first point in endeavouring to photograph it is to ensure that the polished surface is at right angles to the optic axis of the microscope. It will usually happen that metal preparations are irregular in shape, and that only one surface-and that the one under observation-is flat. This therefore must be set up exactly in the position stated. The simplest arrangement for so doing is to lay the specimen with its polished side downwards on to 
an ordinary $3^{\prime \prime} \times 1^{\prime \prime}$ microscope slip. Around this put a metal ring with exactly parallel ends (such metal rings may be obtained commercially in varying sizes and depths for this particular purpose from Messrs. R. \& J. Beck of Cornhill, E.C.), the depth of the ring being greater than the height of the specimen as it lies on the slip. Now place over the specimen some plasticine, putty, or moulders' clay, or some similar easily moulded material. Press down another slip on to the top of this clay so that the object is enclosed between the two slips, these being exactly parallel, and then remove the bottom slip and the metal ring. The object will now be supported on one slip with the polished surface in such a position that it can be placed on the stage of the microscope. This method is simple and inexpensive, and so far as the experience of the writer is concerned, there are few objects that cannot be satisfactorily mounted in this way.

- Various appliances are on the market for holding the specimen and adjusting it exactly at right angles to the optic axis of the microscope without the necessity of embedding it in the manner described. Where fractured unpolished surfaces have to be dealt with, or where the incidence of the light is required to be varied to suit a particular spot in a specimen, these are of considerable value. In general, however, the method of embedding in some soft material is far more useful, as it admits of variation to suit the class of specimen.

If it is desired to preserve a polished surface for future use, then the best preservative is a weak solution of celluloid in amyl acetate. When this is very gently brushed or poured over the surface, it quickly dries off, leaving an extremely thin film which efficiently protects without materially interfering with the corrections of an objective, should it be desired to observe the object again without cleaning the surface. The only advantage in using some of the devices which are made for holding specimens is that they then do not require any mounting; they can in fact be removed from the device or re-introduced at will. There is the disadvantage, however, that on each occasion they have to be re-levelled, and in practice this is often a much longer operation than it would be to embed it again as first described.

For very low-power work, where illumination by directly 
incident light-that is, by light impinging in a direction at right angles to the surface of the specimen-is required, a simple appliance, consisting of a piece of optically worked glass held in front of the objective at an angle of $45^{\circ}$, may be used. In such a case it is better to use objectives corrected to work with covered objects. The image is in this case formed through the reflector, and where an objective is corrected for use without a cover-glass it may possibly result in some deterioration of the image. Since, however, this method is only applicable for use with very low powers it may happen that no serious disturbance results. As to the illuminant, any of the ones already described may be used; but unquestionably the electric arc lends itself best to this class of work. Unless the illuminant is of a high power, it will result in very lengthened exposures. Incidentally also, the difficulty of focussing when the image is projected on the ground-glass screen of the camera, and of ensuring even and efficient illumination, is very much increased unless the illuminant is one of high power. Colour-screens may be used exactly as in work with transmitted light, except that some observation will be necessary to determine the exact effect of the colour-screen in use. When dealing with reflecting surfaces the effect on the image of a given screen is often very different to what it is by transmitted light, and due regard must be paid to this point when setting up the object.

The Photography of Tube- and Plate-cultures of Bacteria.Although this process does not directly come within the scope of photo-micrography, yet it so often becomes necessary to obtain representations of such objects that no apology is offered for giving a description of the methods employed.

The apparatus required consists of an ordinary photographic camera with a suitable lens. The former may be any good camera of the stand or field pattern, and the lens should preferably be of fairly long focus-say from six to eight inches - and will not require to work at a large aperture. It need not be of an expensive type, so that a modern anastigmat is unnecessary, although of course it may be utilised if availabl . All the photographs illustrating this section have been taken with a rapid rectilinear lens of six inches focal length, working at an aperture of $F 8$. 
I The only point that requires special consideration in the photographing of such objects is the means of lighting. In general, tube- or plate-cultures of bacteria may be regarded as either opaque or transparent objects, so that illumination would be conducted as in microscopic objects, either by transmitted or by reflected light. As a compromise a method of oblique lighting is often most useful, and where small semitransparent colonies are lying on a gelatin or agar surface it is generally the only way to obtain any differentiation between the object and the medium.

It is also to be remembered that the two methods of growing bacteria in tubes, usually referred to as stab or streak cultures in which the growth is either in the depth of the medium or on its surface, will require illuminating in different ways. The point to guard against particularly is that, while the surfacegrowth or the growth in depth has to be properly illuminated, it must be done without any light being reflected from the internal or external walls of the glass tube itself, for such reflections interfere seriously with the proper delineation of the object. One method of avoiding reflections from the tube is to immerse it in a rectangular glass vessel which is filled with water, or water and glycerine. With gelatincultures, water will usually be found sufficient, as its refractive index sufficiently nearly approximates to that of the gelatin; but with agar or more highly refractive media, then the water and glycerin combination is better. The exact proportions of the mixture are best determined by observing the effect on the appearance of the tube when more or less glycerin is added to the water; but in general two parts of water to one part of glycerin will be found suitable. With the tubes placed in a glass cell in this manner, it is only necessary to throw an oblique beam from a fairly powerful source of light from a point either in front or behind the tube. The camera is placed in a suitable position in front of the culture, and is so extended that it gives an image which is approximately the same size as the culture-tube itself. The lens will then require to be stopped down to No. F 11 or F 16 to obtain sufficient depth of focus. It may be necessary in order to obtain the best illumination to vary the angle of incidence of the light in a vertical direction so that the light is projected from above the point of view 
or from below, or from one side, as required. Fig. 74 illustrates the relative position of the various parts.

The water-bath will require to be of a fairly large size, so that there is a considerable thickness of fluid all round the tube. One may be easily improvised in the manner already described for making up glass cells to contain the fluids for colourfilters (p. 207).

Between the source of light and the tube a condensing-lens may be placed, but it is advisable to so arrange it that a parallel, or approximately parallel, beam is projected on to the object. In photographing such objects in which there is considerable variation both of colour and general appearance, it is very difficult to give clearly defined instructions, and it is always necessary to do a certain amount of preliminary shifting of the illuminant before a satisfactory result is obtained.

The writer has set up a simple arrangement which may be used for photographing plate- and tube-cultures, and also many other objects of a similar character that may occur from time to time; in fact, any ordinary object-such as a museum specimen-may be photographed with ease by its use (Fig. 74).

It consists essentially of a baseboard, say from three to four feet long and about two feet wide, on one end of which the illuminant $\mathrm{A}$ is fixed. The latter is supported in any suitable manner so that it has considerable range of movement in a vertical direction. At a distance of three or four inches in front of the illuminant an opaque black screen B is placed, and this screen must be varied in width to suit the particular object which is being photographed; in the case of tubes it may be, say, two inches broad, effectively blocking out any direct light that might pass from the illuminant $\mathrm{A}$ to the tube $\mathrm{C}$ placed in the position as shown in the diagram. On each side of the baseboard, and at about six to nine inches' distance from its centre, brass rods EE are fixed, and both these have freedom to rotate about the centre $\mathrm{F}$. On these rods a plane mirror, about eight by six inches-actually a piece of ordinary thin looking-glass - is fixed. Each mirror is supported on a boss which is made to slide up and down the tube, and which also allows the mirror to be inclined at any required angle. It is evident therefore that by altering the position of the mirror, 
or by varying the position of the supporting-rods, the light coming from the illuminant $\mathrm{A}$ may be reflected at any required angle on to either or both sides of the object; or the illumination may be by reflected or transmitted light, as required. No direct light reaches the object from the illuminant owing to the opaque screen which is interposed. A ground-glass screen may be substituted for the opaque screen should directly transmitted light be wanted. This arrangement has the great
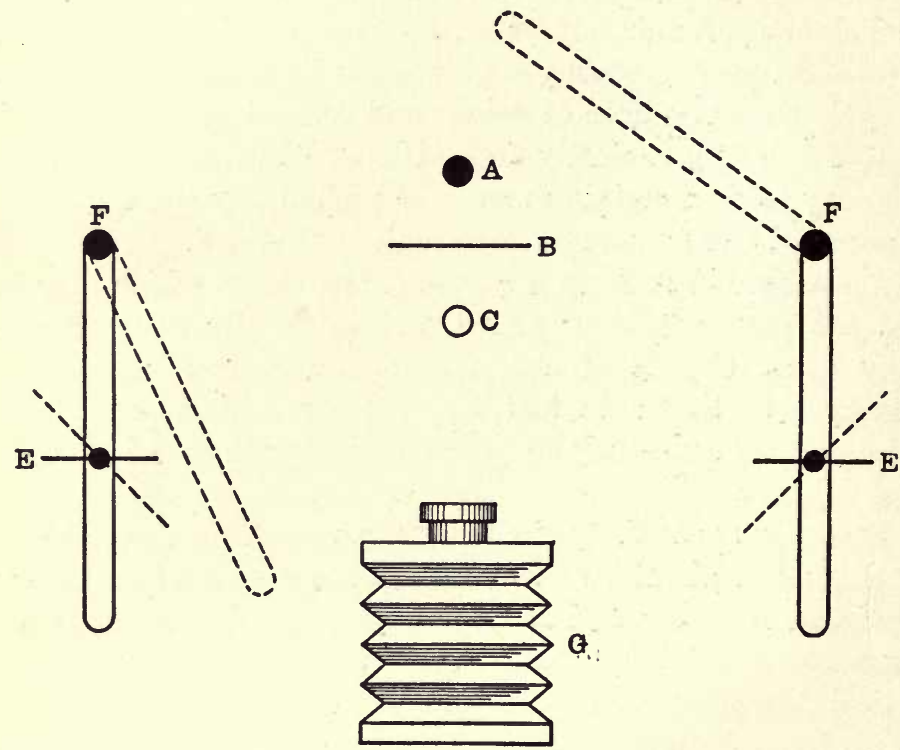

Fic. 74.-Arrangement of Camera and Illuminant for Photographing Tuleand Plate-cultures of Bacteria.

advantage that while the angle of the light may be varied at will, the illumination in certain cases may if required be perfectly even from both sides; that is, if the position of the mirror in relation to the source of light is exactly the same on either side, perfectly even illumination will be obtained. The appliance may therefore be used even for copying diagrams, which are simply placed on the opaque screen and the light reflected on to it. Exactly the same conditions apply in photographing plate-cultures, as these again have either opaque or transparent colonies on them. The opaque ones, however, 


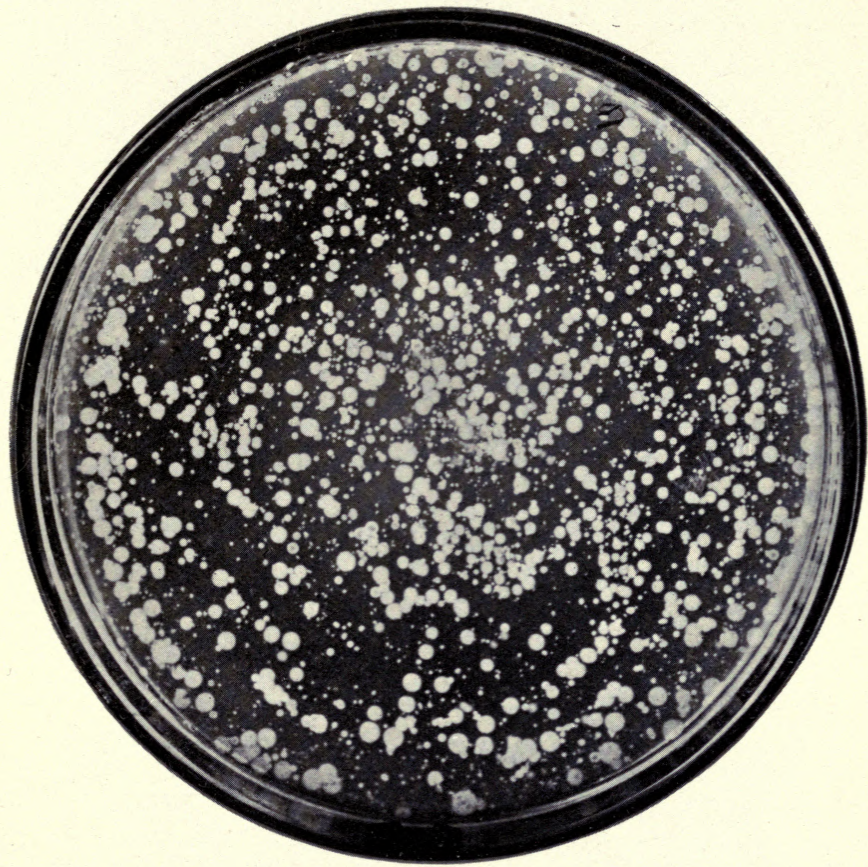

FIG. 75.-Plate Culture of Bacteria (Opaque Colonies). 



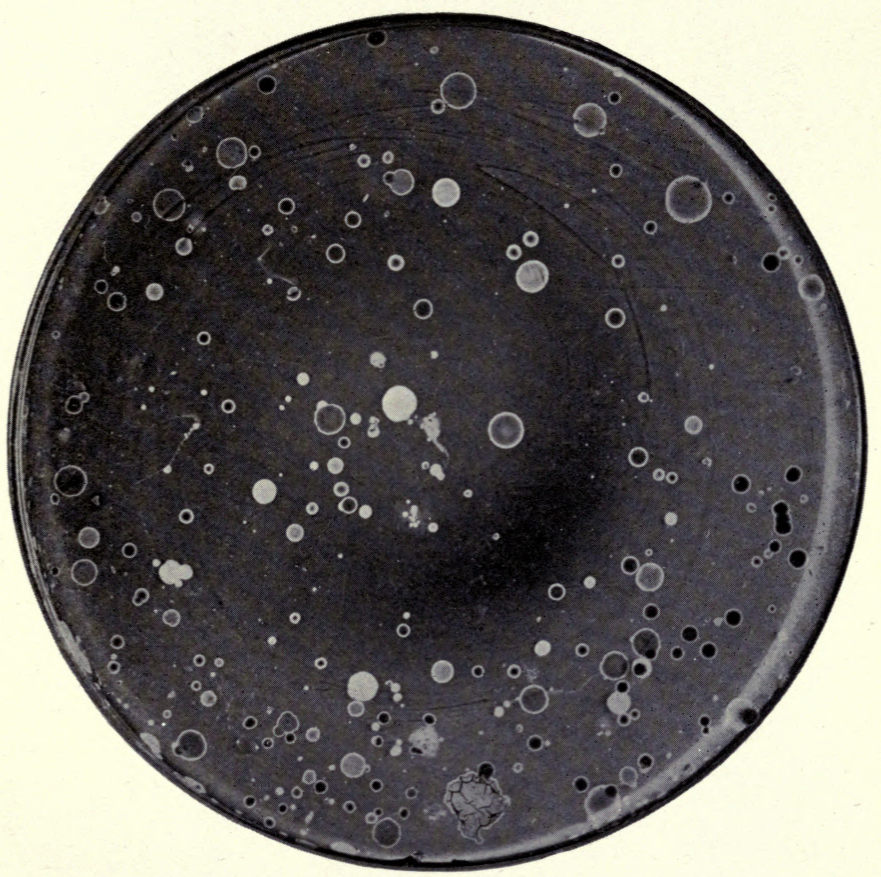

FIG. 76.-Plate Culture of Bacteria (Transparent Colonies). 



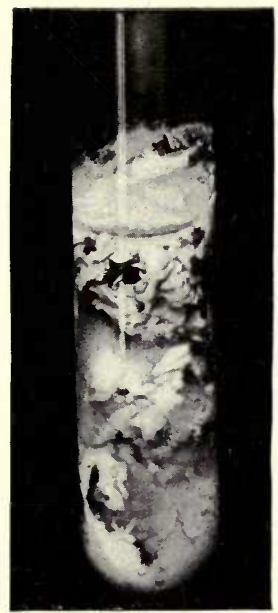

$a$

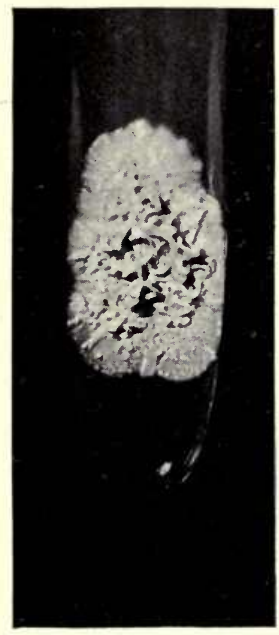

$d$

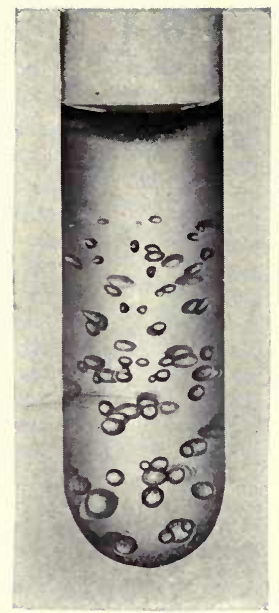

b)

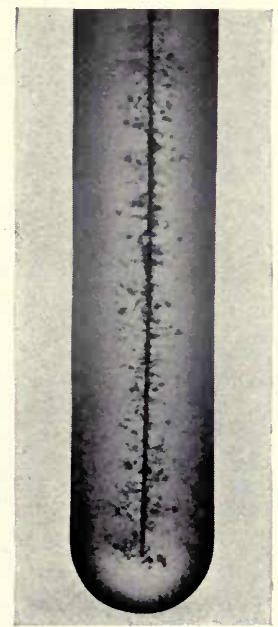

$e$

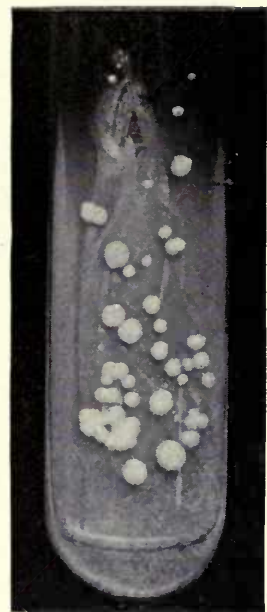

c

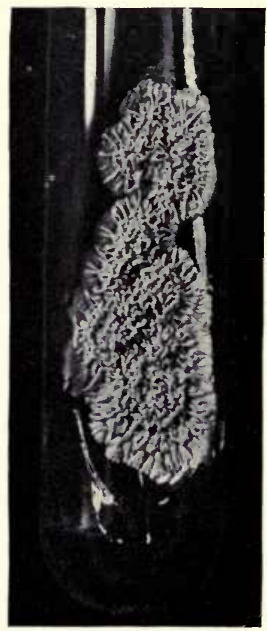

f

Fig. 77.-Tube Cultures of Bacteria. 

should be illuminated by reflecting the light on to their surface (Fig. 75); whereas with those having transparent colonies (Fig. 76) the beam is projected from behind them, but at an oblique angle so that the colonies themselves show up light on a dark background.

Fig. $77 a, b, c, d, e$ and $f$ are examples of tube-cultivations.

a. Coagulation of Milk by Bacillus coli communis. Photographed by reflected light, and with the illuminant placed well below the level of the tube so that reflection from the tube itself was thrown upwards. There is, however, some slight evidence of tube-reflection, a streak showing just near the centre, but it is hardly sufficient to interfere with the result. The culture-tube was not immersed in any tank and shows that it is possible to diminish tube-reflections greatly by suitable adjustment of the light.

b. B. coli communis, Gelatin Shake-culture showing gas-production. Photographed by directly transmitted light. Instead of the opaque screen the ground-glass screen was interposed between the source of light and the tube.

c. Klebs-Loffler or Diphtheria Bacillus on blood serum. Oblique culture with surface colonies, showing very little differentiation so far as the colour is concerned. The colonies, however, have a glistening highly reflecting surface, and by careful arrangement of the light it is possible to bring them out showing brightly on the less bright ground. No water-bath was used in this case, but the result was obtained by reflecting the light from a point well above the level of the tube.

d. Bacillus tuberculosis on agar-agar. Photograph obtained by immersing tube in a water-glycerin bath ; illumination, by reflected light.

e. Bacillus of Tetanus, Stab-culture in sugar-agar. A rather dense agar-tube with growth entirely in depth and at centre of tube. A white screen was placed in the position usually occupied by the opaque screen, and light was reflected from the mirror on to the surface of the tube. The illumination was well above the level of the tube, so that reflections were thrown downwards.

f. Bacillus tuberculosis on agar-agar. Very oblique illumination of a surface-growth on agar. The light was placed at a very acute angle to the surface of the agar in the tube, so 
that the greatest possible contrast was obtained to show up the convolutions in the growth itself.

The exposure required in such cases must of course be dependent on the illuminant; but using a source of lightsuch as a one-ampere Nernst lamp-under the conditions described, none of the exposures for the examples here given exceeded three minutes. A plate of medium rapidity should be used, so that the greatest possible contrast is obtained.

It should be remembered, when using any source of light of high power, that gelatin-cultures particularly will melt down with a relatively small rise of temperature; hence the tubes should be well shielded from any direct light, and if they are contained in a water-bath should not be left long enough for any rise of temperature of the water to take place. In summer even a rise of four or five degrees will be quite sufficient to melt the contents of the tube.

It is also important to remember that if the tubes are contained in a water-bath, the bath itself as well as the outside of the tube should be perfectly clean, as any impurities will show up only too clearly in the photographs.

A condition that sometimes does arise and gives a great deal of trouble is that due to the condensation of water-vapour on the inside of the culture-tube itself. In cases where preparations are permanently sealed the difficulty is a very considerable one. If such condensation appears, it is generally due to the tube being taken for photographing into a room, the temperature of which is lower than that of the incubator in which the growth has been allowed to develop. In certain cases it may be possible to remove the wool plug and by means of a small bit of cotton-wool held in long forceps to wipe the condensed vapour off the inside of the tube. This must be done with the utmost care to avoid disturbance of surface-growth, and in dealing with pathogenic organisms the usual laboratory precautions should be taken. A better way, in cases where the cultures cannot be interfered with, is to place the tube for a short time in a box containing some calcium chloride. In the course of from two to three hours this will usually absorb sufficient moisture to cause evaporation of any free water-vapour in the tube which might otherwise be sufficient to prevent a photograph being taken. 
To reproduce an object in its natural size, the distance of the ground-glass screen of the camera from the lens itself must be double the focal length of the lens (see Appendix IV). This may be determined with sufficient accuracy by taking the focal length of the lens-which is usually marked on the mount-measuring the distance from the iris-diaphragm of the lens to the ground-glass focussing-screen, and making this double the focal length. Obviously, a camera will be required that has sufficient extension, but most half-plate field cameras are long enough. The object must then be placed at approximately the same distance from the lens-diaphragm. In cases where the image is required to be larger than the original, unless the lens is of the symmetrical type, it should be reversed in the camera-front; that is, the back combination of the lens should be placed towards the object, and the image projected with the front of the lens facing the ground-glass screen. In this case no definite instructions can be given as to distance of object or extension of camera, and these must be determined by experiment to suit the particular case. Where symmetrical lenses are used-such as a Zeiss double Prolar-then moderate magnification, up to ten or fifteen diameters, may be obtained with the lens in its normal position in the camera-front.

The appliances already described for dealing with culturetubes and plates may be also used for pathological or similar specimens. In this case, the object may be supported in the position $\mathrm{C}$, and illumination effected as with culture-tubes in which the growth is on the surface. It is sometimes difficult, however, with such objects to support them conveniently in a vertical position, and it is therefore an advantage to have all the appliances described fastened to the baseboard, so that the camera may be supported in the vertical position. The object may then be laid on a glass plate, and illumination effected from above or below as required. With pathological specimens a source of trouble is that they are often in a wet condition, and therefore some parts have a glistening appearance. In such cases they should, if possible, be surface-dried by immersing them in alcohol for a short period. Where this cannot be done, it is often permissible to dust lightly over their surface some fine granular powder, such as powdered 
starch. This does away with the reflecting surface, and may be washed off again immediately the photograph has been taken, without any harm, as a rule, being done to the specimen.

Stereoscopic Photo-micrography. - The production of stereoscopic photo-micrographs is almost entirely confined to low-power work, and the best results are to be obtained with objectives lower than one inch. The process must not be confused with ordinary binocular microscopic vision. It consists essentially of obtaining two photographs side by side on one plate from different points of view in relation to the object, or by means of different zones of the objective. It is also dependent on getting more than one plane of the object in focus ; in fact, as it depends essentially on viewing the object in three dimensions, the focal length of the objective must be considerable; in addition, means are generally taken to restrict the aperture of the objective as far as is optically practicable, to obtain the utmost penetration.

The apparatus in general need not differ from any one already described, except that the dark-slide may be sufficiently large to enable two photographs to be taken on the same plate. There are three methods at present in general use. The first and simplest is to cover up one half of the back combination of the objective by means of a stop; an appliance to do this consists of a short fitting which screws in between the objective and the nose-piece of the microscope. A slot is cut in this fitting, into which is dropped a blackened brass slip, so that exactly one half of the back combination of the objective is covered. One negative is taken through the uncovered half, and then the stop is transferred to the opposite side, and another negative is again taken on the same plate. Prints from these negatives, when suitably mounted, yield a satisfactory stereoscopic effect.

Instead of covering and uncovering the half of the objective, a better method is to place in the same position stops with circular openings. The advantage of this method over the one just described is, as pointed out by Mr. J. Rheinberg, ${ }^{1}$ that owing to the shape of the diffusion-dises in those parts of the object which are not in exact focus being dependent on the shape of the stop at the back of the objective, the diffusiondiscs are at their best with circular openings. There is the

1 Journal of the Queckett Microscopical Club, 1906, pp. 371-396, 
further advantage that both the size and separation of the openings may be varied to suit the particular objects, or to increase or decrease the amount of desirable penetration. The procedure is precisely the same as before, the stop being placed at one side of the objective and then removed to exactly the opposite side to take the succeeding photograph. It is obviously not a matter of moment whether, in relation to the position of the microscope, the stops are side by side or above and below; this will depend simply on the position of the plate in relation to the apparatus.

Mr. H. Taverner ${ }^{1}$ mentions that the size of the circular openings may vary between $2 \frac{1}{2} \mathrm{~mm}$. and $4 \mathrm{~mm}$., and that the inner edge of the aperture will not generally require to be more than $1 \mathrm{~mm}$. from the optic axis of the objective. The size of stop should be the largest possible to give the necessary penetration and to bring out detail sharply in the picture. The inner edge of the aperture should be as near the centre of the objective as will give the necessary stereoscopic effect, while at the same time giving the greatest impression of depth. These circular stops are carried in a suitable fitting at the back of the objective, and may be obtained from Messrs. Baker of High Holborn. Mr. Taverner also recommends the use of a repeating back instead of an ordinary dark-slide. The advantage of this is that when prints are taken from the pictures they are in their proper position relatively to one another for use in a stereoscope-that is, they do not require cutting and remounting. It is important, whichever method is adopted, to see that the apertures are in exact alignment when changed from side to side; otherwise the resulting pictures will not superimpose properly to give the necessary stereoscopic effect.

A third method of obtaining the same result is by shifting the objective or the object by a definite amount from side to side. This is the method advocated by Mr. A. C. Banfield.2 The principle involved is that the inter-ocular distance, normally $62 \mathrm{~mm}$., must be divided by the required magnification to give the angular separation through which the objective or the object must be moved. Thus for 32 diameters the separation

1 Journal of the Royal Microscopical Society, 1906, p. 260.

2 Journal of the Queckett Microscopical Club, 1909, pp. 459-464; Journal of the Royal Microscopical Society, 1910, p. 233. 
required would be nearly $2 \mathrm{~mm}$. In practice it is found advisable to shift the object by the required amount rather than to shift the objective. The degree of necessary separation may be determined by ruling two lines on the focussing-screen at a suitable distance apart, and then moving the object from side to side so that the centre of the field of view coincides with the lines that are ruled on the screen. The exposures are made in succession as in taking ordinary photographs. The objectives most suitable are those of the Zeiss planar type, as their field is flat and their aperture is such that the maximum penetration is attained.

The majority of suitable objects are best illuminated by reflected, rather than by transmitted, light. Any source of light may be used, and exposure will vary within exactly the same limits as in ordinary photo-micrographic work. The illuminating beam may conveniently be a convergent one. There are no unusual practical difficulties to be encountered in this class of work. It is essential to see that in exposing, the two pictures receive exactly the same effective exposure; and that in development the same treatment is given exactly to each. If the two pictures are on the same plate the latter requirement would be complied with in the ordinary course. Where succeeding photographs are taken, it is perhaps best not to employ a high-power source of light, like the electric arc, as there is often considerable variation in the constitution of the carbons, and the actinic power is therefore not always exactly the same for a given period. It is also more difficult with high-power illuminants to give precisely the same exposure, as a very slight variation would cause an appreciable difference in the two negatives. Practically, therefore, it is better to use a source of light such as the Nernst lamp, the mercury-vapour lamp, or acetylene, which is constant, and which entails an exposure of moderate length.

The Production of Lantern-slides in Colour.-The production of lantern-slides in colour has now become such a recognised method, and is, under certain conditions, of such utility, that a short description of the principal available methods should be given. It is, however, only a few objects to which the process is applicable or becomes necessary. With the majority of stained objects, although it increases their appear- 
ance from a pictorial point of view to represent them in colour, yet ordinary photography shows them as well, provided that the detail and differentiation of structure is brought out. Staining methods are themselves arbitrary, and are only employed in order to differentiate structure; therefore, when the structures are photographed, their differences are usually as well shown in monochrome as by any colour method. Coloured lantern-slides of necessity involve a great deal more work in their production ; the results to be obtained are not, from a scientific point of view, usually so exact, and they are therefore not to be recommended except in very special cases. Perhaps in metallography their use is appreciated more than in any other branch of work, as the surface iridescence, or difference in colour to be observed in such specimens, is inherent in the specimen and does not depend on any artificial coloration. In that branch of work, therefore, some of the very best results have been obtained.

The chief methods available are four. The Sanger Shepherd three-colour process (in which three separate negatives have to be taken), the Lumière autochrome process, and the Thames and Dufay colour-plate processes. In the latter three the colour effect is obtained directly on a single plate. It must be admitted that although the first-mentioned process is by far the most laborious one, the results to be obtained by it are from a photo-micrographic point of view superior to any other, as the image is absolutely grainless and is very transparent.

The whole of the necessary materials for carrying out the first process are to be obtained from Messrs. Sanger, Shepherd. $\&$ Co., and full detailed instructions for the work are supplied with the outfit. The method consists essentially in obtaining on a panchromatic plate three separate negatives: one each through a red, green, and violet filter. The exposures to be given will be dependent on the source of light, but need not be of any great length. The time of exposure will not be the same for each screen, but will vary according to the colour-sensitiveness of the plate and the spectroscopic value of the source of light. As an example, with a given illuminant the exposure might be 20 seconds through the red screen, 100 seconds through the green screen, and 25 seconds through the violet screen. It is important to ensure that the relative exposure is correct, 
as otherwise, in case one negative were over-exposed, some spreading action of the light might occur, and its image would tend to be somewhat larger than either of the others.

The most suitable illuminants are those which approximate most nearly to daylight. A source of light, therefore-such as a mercury-vapour lamp-is not available since its spectroscopic value differs so materially from any other. The most suitable illuminants are acetylene, the Nernst electric lamp, or limelight. The arc lamp, perhaps, is not generally so satisfactory, owing to variability in the composition of the carbons, and to the possibility of change in the actinic value of the light with varying voltages and current density, which would disturb the balance so necessary to be maintained between the three negatives.

The negatives are obtained successively through the coloured filters mentioned, and the three resulting negatives are developed in the same solution for the same time so as to obtain as nearly as possible the same type of negative in each case. It need hardly be pointed out that, as the plate used has to be sensitive to practically the whole spectrum, the darkroom illumination must be such that plates of this sensitiveness may be safely dealt with.

Up to this point-the production of the negatives-the process is one that differs in no respect from ordinary photography. From each of these negatives a positive has to be obtained. From the negative taken through the red screen an ordinary lantern-slide on glass is made, and this is then toned to a suitable blue colour with the solution supplied for the purpose by the makers. From the remaining two negatives a positive in bichromated gelatin has to be made. The films, which are coated on celluloid, may be procured ready sensitized from the makers, and should be dealt with exactly in accordance with their instructions. They are printed from the negative in exactly the same way as in obtaining an ordinary lantern-slide, the sensitized surface being placed in contact with the film-side of the negative in an ordinary printing-frame and exposed to a suitable source of light. The exposure must be determined by an actinometer, as it is most important to see that this is correct; but in any case some preliminary experiments may be necessary before the exact duration is arrived at. On removal from the 
printing-frame some indication of the image is seen, and the films must now be placed in water at a temperature of $90^{\circ}$ to $105^{\circ} \mathrm{F}$., until the unaffected portion of the image is dissolved out. This may be carried out in the dark-room, but only an orange or bright-yellow light is necessary. If the image does not appear in relief in a few minutes, but still remains undissolved, the temperature of the water may be raised until the desired effect is obtained. When development is complete, the films are placed in a weak solution of hyposulphite of soda-say 1 in 40 -until all the bromide of silver not acted on is dissolved out, after which they are washed. These two films now have to be stained with the dyeing-solution supplied by the makers, and it is on the exact degree to which this process is carried that success or failure depends. Possibly the best method is to over-stain each of the impressions, and then to immerse them in water and gradually dissolve out the stain until sufficient remains. This method, perhaps, allows of more precise control than an endeavour to remove the films from the stain at the exact moment when they have been treated for a sufficient length of time. A point to be borne in mind is that unless the exposure is strictly correct for each impression, there is a possibility of the images differing somewhat in size, so that on binding them together it is impossible to place them in exact register.

After drying, the films are varnished, and have then to be fastened together by a suitable cement, so that they exactly overlie one another. The instructions on this point, as given by the makers, are sufficiently clear.

To obtain the greatest transparency, the films should be cemented together with Canada balsam, and it is important to ensure that no extraneous matter and no air-bubbles are allowed to get in. The slide is then bound up, and if a mask is desired it is preferably placed on the outside of the slide.

If the whole process has been efficiently carried out, the result is a reproduction in colour which is of perfect transparency and colour-rendering.

The other methods available are essentially different in principle and practice. Instead of taking a separate negative for each of the three primary colour records, the whole colour effect is obtained at one operation and on one plate. Further, no negative is taken but the direct colour positive is given by 
the plate that is exposed in the camera. This is accomplished by coating the plate with a series of filters, which, in the case of the Lumière autochrome method, consists of minute dyed grains of starch; or in the Thames or Dufay colour-plate method, is composed of a series of fine accurately ruled coloured lines or bands. Over either of these screens the emulsion is spread so that the exposure takes place through the combined colour-screen, and is visible on development of the plate. In each of these processes the instructions given by the makers are so complete that it would not appear necessary to repeat them in detail. The points that require special consideration in photomicrography are the source of light and the yellow subsidiary screen through which the photograph is taken. It happens that with daylight, or with any other source of light, that the plates as prepared are still too sensitive to the blue end of the spectrum, so that a yellow-glass screen has to be interposed between the source of light and the object to cut out the unnecessary portion of the light. When daylight is being used these yellow screens, as supplied by the makers, are perfectly efficient; but with artificial light-sources either an additional screen or a specially prepared one becomes necessary. The source of light should approximate as nearly as possible to daylight, and for this reason acetylene is perhaps the most suitable. With this illuminant the ordinary screen supplied by the manufacturers for use with daylight works well. If an ordinary petroleum lamp is used then excellent results may be obtained without using any additional screen; in fact, some of the best results that have passed through the writer's hands have been arrived at by this means.

When using the Nernst electric lamp a special yellow filter must be used. It consists of a yellow and blue screen which are made separately, and then bound together. The formulæ are as follows :-

\section{Yellow Screen.}

Gelatin solution in distilled water, 1 in 15 . 40 c.c.

Tartrazine solution, 1 in 2500 . . . 3 c.c.

Aesculin, dissolved in 37 c.c. of water, with

the addition of 3 drops of ammonia . $0.1 \mathrm{grm}$.

8 c.c. of this solution are used per square decimetre of surface. 


\section{Blue Screen.}

Gelatin solution in distilled water, 1 in 15 . 40 c.c.

Patent blue solution, 1 in 1000 . . . 2 c.c.

7 c.c. of this solution are used for each square decimetre of glass.

The screens are coated with the gelatin solutions, and dried in the manner already described, and are then bound together.

For incandescent gaslight the same formulæ may be used, except that the blue screen is required a little paler, 5 c.c. being used instead of 7 e.c. per decimetre of surface. It must be borne in mind that with either of these sources of light some alteration in their spectroscopic value takes place as they are used. It is better not to use either a Nernst-lamp filament or a Welsbach gas-mantle for a very lengthened period, as their light-efficiency tends to decrease, and the amount of red light they emit increases. When working with the electric arc it must be admitted that difficulties may be encountered. There is the variability in the carbons, and the inevitable variation in the efficiency of the are itself which may affect the results, although in ordinary photographic work this would not become apparent. A screen for use with the electric are may be made as follows :-

Gelatin solution in distilled water, 1 in 15 . 40 c.c.

Tartrazine solution, 1 in 500 . . . 4 c.c.

Saffranine solution, 1 in $7000 \quad$. $\quad$. $\quad$. 1 c.c.

Aesculin, dissolved in 35 c.c. of water, with

the addition of 3 drops of ammonia . $0.4 \mathrm{grm}$.

This solution is suitable for an are taking from 15 to 25 amperes, and 8 c.c. are used for each square decimetre of surface. For ares taking less current, the strength of the solution may conveniently be reduced somewhat so that a screen of less absorptive power is obtained.

A screen suitable for lime-light is as follows :Stock solutions.
A. Quinoline yellow Distilled water .
9.5 grm.
100 c.c.
B. Brilliant acid-green $6 \mathrm{~B}$. . $\quad 0.1 \mathrm{grm}$. Distilled water . . . . . 100 c.c.

In 5 c.c. of distilled water there is dissolved 0.05 gramme 
of aesculin; 0.5 c.c. of solution $\mathrm{A}$ is then added, 1 c.c. of solution $\mathrm{B}$, and 12 c.c. of a 10-per-cent. gelatin solution in distilled water. The whole is made up to 25 c.c. with distilled water, filtered hot, and flowed over the glass in the proportion of 5 c.c. per square decimetre of surface.

These screens are suitable with slight variations for either the Autochrome, Thames, or Dufay plates, but may require some slight alteration to suit the particular illuminant in use. The Autochrome plate is of much finer grain than any other at present on the market; but, fine as it is, lantern-slides made by this process still show, when projected on the screen, some evidence of granularity. The Thames plate is of coarser grain ; but as in the Autochrome plates the starch-grains of a particular colour tend to form themselves into groups, the actual advantage of the Autochrome method over the Thames-plate method is from this point of view not perhaps so great in practice as might be expected from theoretical considerations. The Thames or Dufay plate certainly has somewhat greater transparency; but with either method, when projecting the colour positives, an illuminant must be used of high power, otherwise a brilliant image on the screen cannot be obtained. At present no method is available commercially for producing prints in colour, the lantern-slide or larger positive on glass being all that is practicable. 


\section{CHAPTER XII}

\section{SOME PROGRESSIVE EXAMPLES}

However full any general instructions may be in photomicrographic work, they cannot possibly cover the whole of the varied conditions and circumstances that may arise. It may, therefore, happen that a series of progressive examples, in which various types of objects are selected and various methods of photographing them are described, may prove more useful in particular cases than a treatment of the subject on a broader basis.

All the photographs in Plates I to X are selected not merely as pictorial examples, but as types and varieties that may easily arise in the course of practice. They do not claim to be of necessity the finest that can be produced of a particular subject, but in all cases they are fair examples of what may be expected from an object of average quality. It is a matter of common knowledge that many extremely fine examples of photo micrographic work have been produced not merely as the result of perfect technique, but in large part because the object itself happened to be of unusual quality and suitability. All those here delineated have been specimens of average quality, and such as any ordinary worker may expect to meet with in the usual course.

The description given is generally sufficient to indicate the exact procedure. The only factor that may require variation to obtain the same result is camera-length. It has already been stated that lenses, even of the same nominal focal length, vary within moderate limits in magnifying power. This may be allowed for, and any necessary correction made by variation of the camera-length. Therefore, in some cases where an achromatic objective has been used in which the focal length 
does not accurately indicate its magnifying power, the cameralength has not been mentioned.

The objects are not placed in the absolute order of their difficulty. Some sacrifice has had to be made in this respect to enable them to be reproduced within the limits of the space at disposal. The prints are from untouched negatives.

\section{Plate I, Fig. 1.-Transverse Section of Stem of Clematis. $\times 20$.}

Double stained with carmine and iodine-green.

Objective, $35 \mathrm{~mm}$., Zeiss apochromat (for direct projection without ocular).

Camera-extension, 2 ft. 9 in.

Illuminant, 1-ampere Nernst electric lamp.

No sub-stage condenser.

Convergent beam from Nelson's achromatic bull's-eye, projected so that the image of the radiant was focussed approximately at the surface of the front lens of the objective.

Screen, saturated solution of picric acid.

Exposure, 5 seconds.

Plate, Imperial orthochromatic (extra rapid).

Development by metol-hydrokinone.

Prate I, Fig. 2.-Transverse Section of Stem of Beech. $\times 20$. (Double stained, as above.)

Objective, planar $42 \mathrm{~mm}$., by James Swift \& Son.

All other conditions as in Fig. 1.

Figs. 1 and 2 are photographed under exactly similar conditions to show the difference that exists between ordinary low-power objectives-even those of a high class, such as the apochromatic lenses-and the later types of low powers, such as planars and micro-summars. It will be seen on carefully looking at Fig. 1 that the definition falls off decidedly near the edge of the specimen although the central portion is perfect, whereas in Fig. 2 the definition at the edge is as good as at the centre. Both these photographs are but portions of the original negative, approximately only a quarter of the available field being shown in the reproduction. In photographing such preparations therefore it is necessary to use a lens which has a large field rather than one that possesses corrections of an extremely high order confined to a smaller field. 
Plate I, Fig. 3.-Proboscis of Blow-fly. $\times 100$.

One-inch achromatic objective, N.A., 0*30, by James Swift \& Son ; mediar ocular, 3.

Sub-stage condenser, Swift achromatic with top combination removed. (Working N.A., approximately $0 \cdot 28$.)

Auxiliary condensers, Zeiss Nos. I and III.

Illuminant, mercury-vapour lamp.

Screen, Gifford F-line.

Camera-extension, $2 \mathrm{ft}$. 6 in.

Plate, Wellington orthochromatic process.

Exposure, $1 \frac{1}{2}$ minutes.

Development by pyro-soda, with half the normal quantity of pyro in solution, to reduce as far as possible contrast in the dense part of the preparation.

This object is always regarded as a particularly good test with low-power objectives. The illumination should be effected with a cone of light as large as the objective will allow, and all corrections must be most carefully made to ensure that the fine spirals and the outlying hairs on the tongue are brought out as perfectly as possible. The hairs themselves should, where they are in focus, die away to a perfectly sharp welldefined point. It is necessary to select a tongue that is mounted as flat as possible, and that is not too dense in its densest parts; otherwise it is impossible to obtain detail in these parts of the object without lessening that to be seen in the thinner parts. A well-corrected objective will not of necessity require any colour-screen; but the object is usually such that the use of one is beneficial in helping to bring out detail. Its colour is usually so non-actinic that it may almost be regarded as an object in black-and-white, presenting no colour difficulty, when orthochromatic plates are used.

Plate II, Fig. 1.-Group of Foraminifera. $\times 25$. Opaque object.

Objective, $35-\mathrm{mm}$. apochromat for direct projection, by Carl Zeiss.

No ocular.

Camera-extension, 2 ft. 9 in.

Illuminant, 4-ampere electric arc lamp, by Leitz.

The object is mounted as an opaque one, and illumination therefore has to be effected by obliquely incident light. The 
illuminant was arranged in a position to one side of and about fifteen inches away from the preparation, so that a beam of light at about an angle of $45^{\circ}$ to the normal was projected on to it. An ordinary bull's-eye condenser was interposed, so that an enlarged image of the illuminant was roughly focussed on to the preparation.

Exposure, $1 \frac{1}{2}$ minutes.

Thomas's lantern-plate.

Development with pyro-ammonia, Thomas's formula.

Such low-power opaque objects present no particular difficulty, except that the preparation should be mounted so that all the units lie in as nearly as possible the same plane. In cases where the object is one of considerable depth, it is better to have recourse to a lens of the planar type which has an iris-diaphragm. This allows the aperture to be reduced, with resulting increase of penetration. Alternatively, a Davis diaphragm may be placed at the back of the micro-objective, and this can be closed down as required, care being taken that the quality of the image is not interfered with. In any case, for reasons already stated, the lens should be of the lowest power possible.

It may be necessary to vary the angle of incidence of the light according to the depth of the surface-markings on each individual unit of the preparation. The nearer the incidentbeam approaches to the vertical, the less the contrast that will be obtained. It is, however, always advisable to keep the incident beam at as small an angle to the vertical as practicable; otherwise heavy shadow effects are caused on one side, which interfere seriously with correct delineation of the object.

Plate II, Fig. 2.-Transverse Section of Stem of Sarsaparilla. $\times 50$. Stained with Bismarck brown.

(Owing to the colour of this stain, this object may be regarded as one which presents no colour difficulty in photographing, for the colour of the image itself is non-actinic to any ordinary plate.)

Objective, 1 inch, by C. Baker.

No ocular.

No sub-stage condenser.

Illuminant, an acetylene bicycle lamp. Ordinary bull'seye condenser projecting a convergent beam, and so placed 
that a roughly defined image of the illuminant is projected approximately in the position of the front lens of the objective, the object occupying a position within the apex of the illuminating-cone.

Camera-extension, $3 \mathrm{ft} .6$ in.

Plate, Edward's Isochromatic (extra rapid).

Exposure, 30 seconds.

Development, metol-hydrokinone, somewhat restrained by the addition of a few drops of a $10 \%$ solution of bromide of potassium to ensure a black-and-white result. Such an object may be regarded as of the easiest type, and is eminently suitable for a beginner.

Plate II, Fig. 3.-Spine of an Echinus. $\times 40$.

Unstained preparation.

Objective, 1-inch achromatic, by R. \& J. Beck.

No ocular.

Camera-extension, 3 ft. 2 in.

Small tube of black paper slipped into body-tube of microscope to prevent reflection from the internal surfaces.

Dark-ground illumination obtained by putting a stop of suitable size at the back of the achromatic condenser, the top lens of which had been removed.

Illumination by Welsbach incandescent gaslight, the source of light being placed about eight inches from the sub-stage condenser.

No colour-screen.

Ilford ordinary landscape plate.

Exposure, 2 minutes.

Development by pyro-soda, the utmost contrast being secured by using an excess of pyro, and about $10 \%$ less than the normal quantity of carbonate of soda.

Plate II, Frg. 4.- Section of Human Scalp. $\times 75$.

Preparation stained with hæmatoxylin and methylene-blue.

Objective, R. \& J. Beck's $\frac{1}{2}$-inch achromatic.

No ocular.

No sub-stage condenser.

Illumination by a round-wick oil lamp.

Nelson's achromatic bull's-eye projecting an image of the source of light on to the specimen. 
Camera-extension, $3 \mathrm{ft}$.

Plate, Wratten \& Wainwright's Allochrome.

Screen, signal-green glass.

Exposure, 3 minutes.

Development by amidol. The preparation presents rather violent colour-contrast, so this developer was used to obtain a soft negative. As the contrast between the two colours with which the object is stained is photographically considerable, the green-glass screen gives good results owing to its general absorptive power on either side of the green region in the spectrum.

Plate III, Fig. 1.-Transverse Section of Human Tongue. $\times 50$.

Stain, hæmatoxylin.

Objective, Zeiss 16-mm. apochromat.

Projection-ocular, 2.

Illumination, acetylene bicycle lamp.

Condenser, back combination of Swift achromatic condenser, working at its full N.A.

Auxiliary condenser, Nelson achromatic.

Screen, saturated solution of picric acid.

Camera-extension, 20 in.

Plate, Imperial orthochromatic (extra rapid).

Exposure, 15 seconds.

Development, metol-hydrokinone.

Plate III, Fig. 2.-Transverse Section of Stem of Black Pepper. $\times 30$.

Specimen double stained with iodine-green and fuchsin.

This specimen was an old one, and had faded very considerably, so that the object was faintly seen, and the colours were not well differentiated.

Objective, 24-mm. achromatic, by E. Leitz, Wetzlar.

No ocular.

Illuminant, Nernst electric, taking 1 ampere.

No sub-stage condenser.

An enlarged image of the filament projected on to the object by means of Zeiss Nos. I and III collecting-lenses.

Screen, bichromate of potash and copper acetate, the bichromate of potash being in excess, so that the screen was of a deep yellowish-green colour. 
Camera-extension, $2 \mathrm{ft} .6$ in.

Exposure, $1 \frac{1}{2}$ minutes.

Development by pyro-soda.

It will be seen that, in spite of the faint staining of the specimen, the use of a suitably adjusted screen of deep colour has enabled the differentiation of structure to be well brought out; in fact, judging from the photograph, there is no indication that the specimen was not a good one.

Plate III, Fig. 3.-Group of Foraminifera. $\times 25$.

Transparent object.

Objective, 1-inch achromatic, by W. Watson \& Sons.

No ocular.

Illuminant, electric are, taking 10 amperes.

No sub-stage condenser.

Illumination by slightly convergent beam projected by Nelson's achromatic bull's-eye.

Screen, filter-yellow $\mathrm{K}$ and tartrazine.

Camera-extension, $1 \mathrm{ft} .9$ in.

Plate, Wellington orthochromatic process.

Exposure, 2 seconds.

Development with pyro-soda, somewhat restrained with potassium bromide to ensure maximum contrast.

Such an object presents no particular difficulty, except that it is advisable to use a lens of as long focus as possible, and with the smallest working N.A. that will bring out the required detail, thus ensuring maximum penetration.

Plate III, Fig. 4.-Medulla of Monkey. $\times 15$.

Staining, Weigert hæmatoxylin.

Objective, Zeiss 75 -mm. micro-planar, mounted on front of camera.

No ocular.

Illuminant, Leitz electric arc, taking 4 amperes.

Sub-stage condenser, Watson's macro-illuminator.

Convergent-beam projected into condenser by means of ordinary bull's-eye as supplied with Leitz electric lamp.

Screen, saturated solution of auramin.

Plate, Wellington orthochromatic process.

Exposure, 10 seconds.

Development, pyro-soda. 
Plate IV, Fig. 1.-Impression Preparation of Bacillus anthracis. $\times 100$.

Stain, methylene-blue.

Objective, $\frac{1}{4}$-inch achromatic, by Charles Baker.

No ocular.

Illuminant, Nernst electric lamp, taking 1 ampere.

Condenser, Abbe chromatic, working at full aperture. Auxiliary condenser, Zeiss Nos. I and II collecting-lenses.

Screen, saturated solution of bichromate of potash.

Camera-extension, $2 \mathrm{ft}$.

Plate, Barnet orthochromatic.

Exposure, 40 seconds.

Development, pyro-soda.

Plate IV, Fig. 2.-Colony of Bacillus coli communis, on Gelatin Plate. $\times 50$.

Objective, 1-inch achromatic, by W. Watson \& Sons.

No ocular.

Illuminant, electric arc, taking 10 amperes.

No sub-stage condenser.

Oblique illumination obtained by projecting a slightly convergent beam on to the mirror of the microscope, the latter being thrown well to one side.

Screen, Gifford F-line.

Camera-extension, $3 \mathrm{ft}$.

Plate, Wratten \& Wainwright's lantern.

Exposure, 30 seconds.

Development by metol-hydrokinone.

In photographing such an object in order to bring out the reticulated surface-structure suitable obliquity of the light is the essential point, and this can only be arrived at by experiment. The beam should first be projected at the smallest possible angle to the optical axis, and this angle gradually increased until the desired effect is obtained. The least possible obliquity of the beam should be used that will show up the structure, to avoid the introduction of diffraction effects that would otherwise interfere with the formation of a good image.

Plate IV, Fig. 3.-Group of Mixed Diatoms. $\times 100$.

Objective, $\frac{2}{3}$-inch, by James Swift \& Son. 
No ocular.

Illuminant, one-ampere Nernst lamp.

Zeiss auxiliary collecting-lenses, Nos. I and III.

Watson achromatic condenser with top lens removed.

Critical image of auxiliary iris-diaphragm projected into the object-plane.

Green screen, acetate of copper and bichromate of potash ; the acetate of copper being in excess so that the screen is visually of a bright green, transmitting no red light.

Camera-extension, $3 \mathrm{ft}$. 6 in.

Exposure, 10 seconds.

Plate, Wellington orthochromatic process.

Illuminating-cone to fill two-thirds of the back lens of the objective.

Development by pyro-soda, with pyro in excess, and five drops of $10 \%$ bromide of potassium to each ounce of developer.

These objects present no particular difficulty, and may be satisfactorily photographed with any medium-power achromatic lens, provided the colour-screen used completely absorbs all light of short wave-length. In developing, it is important to see that contrast is secured, as the print should show a pure black-and-white effect.

Plate IV, Fig. 4.-Surface Colony of Ringworm Organism. $\times 2$.

Objective, $6 \frac{1}{4}$-inch Zeiss double-prolar anastigmat, on camera-front.

Illuminant, lime-light. A parallel beam projected on to surface of plate by ordinary bull's-eye, at an angle of $45^{\circ}$ to the optical axis.

Screen, green pot-glass.

Plate, Edward's isochromatic, medium rapidity.

Exposure, 10 seconds.

Development, pyro-soda.

Plate IV, Fig. 5.-Surface Colony of Bacillus anthracis. $\times 20$.

Objective, Leitz 75-mm. micro-summar.

No ocular.

Illuminant, lime-light. 
Condenser, Swift achromatic, with top combination removed, and central stop to obtain dark-ground illumination.

Parallel beam projected into condenser by means of ordinary bull's-eye.

Screen, green pot-glass.

Plate, Edward's isochromatic, medium rapidity.

Exposure, 30 seconds.

Development, pyro-soda.

Plate V, Fig. 1.-Tripanosoma gambiense. $\times 450$.

Stained by Romanowsky-Leishman method. (Methyleneblue-eosin.)

One-quarter-inch objective, by James Swift \& Son.

Projection-ocular, 4.

Camera-extension, 2 ft. 9 in.

Illuminant, one-ampere Nernst lamp.

Zeiss auxiliary collecting-lenses Nos. I and III, projecting an enlarged image of the illuminant on to the sub-stage irisdiaphragm.

Leitz oil-immersion condenser with top removed, working therefore as a dry condenser of relatively low aperture, but sufficient N.A. to completely fill the back lens of the objective.

Green screen composed of two separate solutions of acidgreen and tartrazine in cells $5 \mathrm{~mm}$. thick. The specimen being double stained red and blue, this ensures the utmost contrast.

Exposure, 30 seconds.

Plate, Edward's isochromatic, medium speed.

Development by pyro-soda, but rather weak in pyro to bring out the utmost detail in the body part of the organisms. These, and similar organisms, should be treated much in the same way as in photographing bacteria. The largest possible illuminating-cone should be used to ensure that the flagellø are brought out as fine and sharp as possible.

Plate V, Fig. 2.-Diatom: Orthonies splendida. $\times 500$. Objective, $\frac{1}{6}$-inch, dry, No. 6 (N.A., 0.85) ; tube-length, $160 \mathrm{~mm}$. by C. Reichert, Vienna. (This objective was one of a series, placed at the disposal of the writer by the courtesy of Messrs. H. F. Angus \& Co. of Wigmore Street. London.) 
Zeiss projection-ocular, 2.

Camera-length, 2 ft. 10 in.

Illuminant, mercury-vapour lamp.

Sub-stage condenser, Swift apochromatic, N.A., 0.95.

Auxiliary condenser-plano-convex lens, with plain surface towards the illuminant, the image of the illuminant being focussed at the plane of the sub-stage iris-diaphragm. Auxiliary iris-diaphragm interposed between the illuminant and auxiliary lens. Critical image of the opening in the latter irisdiaphragm projected into the objective plane. Sub-stage iris closed, so that about two-fifths of the total aperture of the objective was used.

Plate, Wellington process orthochromatic. Tartrazine and filter-yellow-K fluid screen. The whole of the blue-violet and ultra-violet light emitted by the mercury-vapour lamp eliminated.

Exposure, $1 \frac{1}{2}$ minutes.

Development by pyro-soda. A developer containing rather more than the usual quantity of pyro, and a few drops of a $10 \%$ solution of potassic bromide were used to secure contrast.

As with all diatoms, the important point is to see that the sub-stage iris-diaphragm is not closed sufficiently to produce diffraction images around the outer edge of the frustule, or around the secondary markings.

Plate V, Fig. 3.-Tripanosoma gambiense. $\times 1000$.

Stained by Romanowsky-Leishman method. (Methyleneblue-eosin.)

One-twelfth-inch objective, by C. Baker.

No ocular.

Short thick velvet sleeve inserted in top of microscope bodytube to stop reflection.

Abbe chromatic condenser.

Plano-convex auxiliary lens giving a parallel beam.

Illuminant, mercury-vapour lamp.

Green screen as in Plate V, Fig. 1.

Plate, Barnet orthochromatic.

Exposure, 5 minutes.

Development with pyro-soda. 


\section{Plate V, Frg. 4.-Cover-glass Preparation of Pigeon's Blood. $\times 360$. \\ Double-stained eosin and methylene-blue. \\ Objective, 16-mm. apochromat, by Carl Zeiss. \\ Projection-ocular, 2.}

Illuminant, round-wick paraffin lamp. Abbe chromatic sub-stage illuminator. The illuminant was placed about ten inches from the sub-stage condenser, and no intervening condensing-lens employed, the image of the radiant itself being sufficiently large to cover the field. The illuminating-cone was reduced so that about two-thirds of the N.A. of the lens was employed.

Green screen composed of acetate of copper and chromic acid, the acetate of copper being in excess. The source of light was one of low intensity, so that the solutions employed were diluted to half-saturation strength. The thickness of the cell containing the fluid was $2 \mathrm{~mm}$. only, to reduce general absorption of light.

Exposure, 2 minutes.

Plate, Barnet rapid orthochromatic.

Development by pyro-soda, the proportion of pyro being kept somewhat below the normal to ensure detail in the body of the cell being brought out. Such objects generally present no special difficulty, provided they are prepared as cover-glass preparations, and the staining is not too deep.

Plate V, Fig. 5.-Diatom : Navicula lyra. $\times 600$.

Objective, $\frac{1}{4}$-inch, by J. Swift \& Son; mediar ocular, 3.

Illuminant, arc lamp of the type already described, with pin-hole indicators and vertical carbons. Current, 10 amperes. Achromatic oil-immersion condenser used dry. Slightly convergent beam from a plano-convex lens projected into the back lens of the condenser. A critical image of large irisdiaphragm, the latter placed between the plano-convex lens and sub-stage condenser, projected into the object-plane. A two-thirds cone of light employed.

Screen, Zettnow's green filter; thickness, $10 \mathrm{~mm}$.

Exposure, 2 seconds.

Wellington orthochromatic process plate.

Development by pyro-soda, with an increase in the 
pyro of about $20 \%$ to ensure the utmost contrast. The aim in developing should be to get an image as nearly as possible black-and-white. It is necessary in photographing this diatom to have an objective of sufficient N.A. to bring out the dotted structure, but the illuminating-cone must even then be reduced. It is important to observe carefully the image while reducing the cone, to make sure that the dotted structure is not lost and that no diffraction-effects begin to show themselves around the dots.

Plate V, Fig. 6.-Unstained Living Yeast-cells. $\times 500$.

Objective, 6-mm. apochromat, by C. Zeiss, corrected for 250-mm. tube.

Projection-ocular, 4.

Vertical camera of the Van Heurck type, with fixed extension.

Newman \& Guardia reflex camera placed as described on p. 140, so that direct observation of the image could be carried out up to the moment of exposure.

Illuminant, electric arc, taking 10 amperes.

Leitz oil-immersion sub-stage condenser.

The illuminating-cone had to be reduced to approximately one-half of the total N.A. of the objective to obtain as great a contrast as possible ; the object itself being transparent and depending for its visibility on the difference of refractive index between the cell body and the medium in which the organisms lie.

Screen, $1 \%$ solution of methylene-blue, $2 \mathrm{~mm}$. thick.

Plate, Imperial flashlight, ordinary.

Exposure, one-twenty-fifth of a second by means of focal plane shutter.

Development by methol-hydrokinone, with an excess of hydrokinone to obtain contrast. Such preparations should be mounted not in hanging drop, but in as thin a film as possible between an ordinary slide and cover-glass. The chief difficulty lies in getting a field of organisms which all lie in the same plane.

Plate VI, Fig. 1.-Actinomycosis bovis. Section of Tongue of Cow. $\times 350$.

The organism stained Gram, and counter-stained eosin.

Objective, $\frac{1}{4}$-inch achromatic, by Charles Baker.

Ocular, none. 
Illuminant, Welsbach incandescent gas.

Condenser, achromatic.

No auxiliary condenser.

Screen, saturated solution of auramine, $1 \mathrm{~mm}$. thick.

Camera-extension, $5 \mathrm{ft} .9$ in.

Plate, Imperial orthochromatic.

Exposure, 4 minutes.

Development, pyro-soda.

The chief difficulty in photographing such a preparation arises from the fact that the sections are seldom thin, and therefore several planes have to be brought into approximate focus at the same time. The only way of successfully dealing with the matter is to reduce the aperture of the sub-stage condenser as far as practicable without loss of definition. It therefore results, as shown in this photograph, that no part of the object is in what might be called critical focus, a compromise having to be effected so that only moderately good definition can be obtained.

Plate VI, Frg. 2.-Bacillus pestis in Blood (Bubonic Plague). $\times 750$.

Stain, methylene-blue.

Objective, $\frac{1}{15}$-inch oil-immersion, by C. Reichert.

Ocular, none.

Illuminant, oil lamp.

Condenser, Swift achromatic.

No auxiliary condenser.

Screen, filter-yellow-K, $1 \mathrm{~mm}$. thick.

Plate, Imperial orthochromatic.

Exposure, 6 minutes.

Development, pyro-soda.

Plate VI, Fig. 3.-Bacillus tuberculosis. Giant Cell in Lung. $\times 1000$.

Stain, Ziehl-Neelsen carbol-fuchsin and methylene-blue. Objective, $\frac{1}{12}$-inch oil-immersion, by W. Watson \& Sons.

Ocular, none.

Illuminant, blow-through lime-light jet.

Condenser, Swift achromatic.

No auxiliary condenser.

Camera-length, 5 ft. 6 in. 
Screen, signal-green glass.

Plate, Wellington orthochromatic process.

Exposure, $1 \frac{1}{2}$ minutes.

Development, pyro-soda.

Plate VI, Fig. 4.-Koch's Spirillum of Cholera. $\times 1500$. (Cover-glass preparation of a pure culture.)

Stain, fuchsin.

Objective, 3-mm. apochromat, by Carl Zeiss.

Ocular-projection, No. 2.

Illuminant, arc lamp, taking 4 amperes.

Condenser, Leitz oil-immersion N.A. 1·40.

Auxiliary condenser, Nelson achromatic bull's-eye.

Screen, iodine-green saturated solution.

Plate, Wellington orthochromatic process.

Exposure, 45 seconds.

Development, pyro-soda.

Plate VI, Fig. 5.-Human Hair, showing the Organism of Ringworm (Microsporon audouini) on its surface. $\times 450$. Unstained. The hair had been macerated in caustic potash to get rid of some portion of the hair structure and to increase transparency.

Objective, $\frac{1}{4}$-inch, by J. Swift \& Son.

Ocular, none.

Illuminant, electric arc, taking 4 amperes.

Condenser, Zeiss-Abbe illuminator.

Auxiliary condenser, Nos. I and II.

Screen, methylene-blue, aqueous solution.

Plate, Wellington orthochromatic process.

Exposure, 40 seconds.

Development, by metol-hydrokinone.

The whole difficulty with such a preparation is to obtain sufficient contrast, as the organism itself is very transparent. This can only be effected by reducing the aperture of the substage condenser, so that a narrow illuminating-cone is used. The condenser aperture must be reduced carefully to ensure that no diffraction rings appear round the organism or at the edge of the hair itself.

Plate VII, Fig. 1.-Bacillus anthracis. Cover-glass Preparation of Blood of Inoculated Guinea-pig. $\times 750$. 
Stain, Gram and eosin.

Objective, $\frac{1}{15}$-inch oil-immersion, by C. Reichert.

Ocular, none.

Illuminant, mercury-vapour lamp.

Condenser, Swift achromatic.

No auxiliary condenser.

Screen, acid-green and tartrazine.

Plate, Barnet orthochromatic.

Exposure, 45 seconds.

Development, pyro-soda.

Plate VII, Fig. 2.-Spirochaete pallida. $\times 1500$.

Staining by Gemse's method.

Objective, 3-mm. apochromat, N.A. 1·40, by Zeiss.

Ocular-projection, No. 2.

Illuminant, arc lamp, taking 4 amperes.

Condenser, Leitz oil-immersion, used dry.

Auxiliary condenser, Zeiss No. I.

Screen, saturated solution of auramine.

Plate, Wellington orthochromatic process.

Exposure, 65 seconds.

Development, metol-hydrokinone.

This preparation was extremely faintly stained, and it was therefore difficult to get sufficient contrast in the photograph. It is one of a class in which the negative might usefully be intensified by one of the methods described on p. 235, although the figure reproduced has not been so dealt with.

Plate VII, Fig. 3.-Streptococcus pyogenes. $\times 1250$. Cover-glass preparation from pure culture.

Stain, methyl-violet.

Objective, $\frac{1}{12}$-inch achromatic, by C. Baker.

Ocular, none.

Illuminant, electric arc, taking 4 amperes.

Condenser, Zeiss-Abbe chromatic.

Auxiliary condenser, Nelson aplanatic bull's-eye.

Screen, aqueous solution of naphthol-green.

Plate, Wellington process, orthochromatic.

Exposure, 6 seconds.

Development, pyro-soda. 
Plate VII, Fig. 4.-Bacillus typhosus. Cover-glass Preparation showing Flagella. $\times 1250$.

Staining by Van Ermengem's method.

Objective, 2-mm. apochromat, N.A. 1·40, by Carl Zeiss (corrected for 250-mm. tube-length).

Ocular-projection, No. 2.

Illuminant, electric arc, taking 10 amperes.

Condenser, Swift achromatic.

Auxiliary condenser, Zeiss No. I.

Screen, Gifford F-line.

Plate, Barnet orthochromatic.

Exposure, 7 seconds.

Development, pyro-soda.

Plate VII, Fig. 5.-Micrococcus gonorrhoea. Cover-glass Preparation of Pus. $\times 1500$.

Stain, Loffler's methylene-blue and eosin.

Objective, 3-mm. apochromat, N.A. 1·40, by Carl Zeiss.

Projection-ocular, No. 2.

Illuminant, lime-light, mixed jet.

Condenser, Leitz oil-immersion, used dry.

Auxiliary condenser, Zeiss No. I.

Screen, filter-yellow-K and eosin.

Plate, Imperial orthochromatic.

Exposure, 45 seconds.

Development, pyro-soda.

The main difficulty in dealing with such objects, where micro-organisms are lying in or on blood-cells, is to get sufficient depth of focus. The objective used should be of the largest focus that will show the desired structure, as great resolving power is not necessary. Magnification may therefore be obtained by increased ocular-power, or greater camera-length. The sub-stage iris-diaphragm may be contracted to about half its full working aperture.

Plate VII, Fig. 6.-Bacillus of Leprosy in Skin. $\quad \times 1250$. Stain, fuchsin and methylene-blue.

The same conditions apply as in Fig. 5, and the same difficulty of obtaining a number of bacilli in the tissue in depth in proper focus will be met with. Such sections are usually not very thin, so that the trouble is further accentuated and can only be met in the manner suggested. 
Plate VIII, Fig. 1.-Diatom : Navicula lyra. $\times 850$. $170 \mathrm{~mm}$.)

Objective, 4-mm. apochromat, by E. Leitz. (Tube-length,

Ocular-projection, No. 4.

Mluminant, lime-light, mixed jet.

Condenser, Swift apochromatic, N.A. 0·95.

No auxiliary condenser.

Screen, Gifford F-line.

Plate, Wellington process orthochromatic.

Exposare, 35 seconds.

Development, pyro-soda.

Plate VIII, Fig. 2.-The Podura Scale (Lepidocyrtus curvicollis). $\times 1750$.

Objective, $\frac{1}{12}$-inch achromatic. (Tube-length, $168 \mathrm{~mm}$.) By J. Swift \& Son.

Ocular, Swift mediar No. 3.

Illuminant, electric arc, 4 amperes.

Sub-stage condenser, Swift apochromatic stopped down to one-third cone.

Auxiliary condenser, Zeiss No. I.

A parallel beam was projected into the sub-stage condenser by means of the auxiliary condenser, and a critical image of the iris-diaphragm in front of the auxiliary condenser projected into the object-plane.

Screen, Zettnow's fluid filter, $6 \mathrm{~mm}$. thick.

Plate, Wellington orthochromatic process.

Exposure, 45 seconds.

Development, pyro-soda.

This object has already been mentioned as being the recognised optician's test for adjusting objectives. To obtain a perfectly defined photograph of it, the objective must be of the finest-preferably, apochromatic; but if a suitable greenglass screen is used, such as Zettnow's filter already described, or a signal-green and pale-yellow glass combined, a good achromatic oil-immersion objective will suffice.

The point to bear in mind in attempting this object is that the internal exclamation marks are as clearly shown as possible; they should have a knoblike end, and then taper off from that to a fine point. A one-eighth-inch oil-immersion objective would show these equally well. The object is a test for 
achromatism rather than for any other quality, as the N.A. required to bring out the desired appearance is not high.

It is essential that this object should be mounted dry, and that the surface to be photographed should be in optical contact with the cover-glass. Such preparations are at the present time scarce, and good ones appear to be becoming ever more difficult to obtain.

Plate VIII, Fig. 3.-The Podura Scale (Lepidocyrtus curvicollis). $\times 2500$.

Enlargement made from Fig. 2.

The method adopted was to make a positive on a backed Wratten \& Wainwright lantern-plate, and from this to make an enlarged negative, and to print direct from the latter.

Plate VIII, FIg. 4.-Diatom : Pleurosigma angulatum. $\times 1000$.

Objective, 6-mm. apochromat, by Carl Zeiss. (Tube-length, $250 \mathrm{~mm}$.)

Ocular, compensating, No. 8.

Illuminant, electric arc, taking 10 amperes.

Screen, filter blue-green.

Plate, Imperial orthochromatic.

Exposure, 20 seconds.

Development, metol-hydrokinone.

This difficult and much-discussed diatom may be photographed to show varying appearances. These appearances are to a great extent dependent on the focus at which the photograph is taken, as by focussing up or down on the object either a black- or white-dot image may be obtained. In the particular portion of the frustule shown it will be seen that on one side the black-dot image is seen, whereas on the other the white-dot appears. In places some intermediate stages are shown.

Plate IX, Fig. 1.-Diatom: Amphipleura pellucida. $\times 1500$.

Preparation mounted in realgar.

Objective, 2-mm. apochromat, N.A. 1.40 (corrected for 250-mm. tube-length), by Carl Zeiss.

Ocular, No. 4 projection.

Illuminant, electric arc, 10 amperes.

Condenser, Leitz oil-immersion, N.A. 1·40. 
Auxiliary condenser, Zeiss No. I.

Screen, aqueous solution of methylene-blue with signalgreen glass to cut out the red otherwise transmitted.

Plate, Imperial orthochromatic.

Exposure, 4 minutes.

Development with metol-hydrokinone.

The illumination to bring out the lined structure requires to be oblique. This may be obtained by putting a slotted stop at the back of the sub-stage condenser in the manner already described; or by partially closing the iris-diaphragm and racking it across the field (as may be done in Continental forms of stands) so that only a small portion of the total aperture of the condenser, and that at one side only, allows light to pass. The light must pass through the condenser and impinge on to the object in the direction of the length of the latter. The exact amount of decentration of the sub-stage iris must be determined by experiment and depends to some extent on the character of the particular frustule in view. The largest opening and the least amount of decentration possible should be used, but the lines must be shown clear and bright. The black lines should not exceed in width one-fourth of the width of the intervening clear spaces. The result obtained depends greatly on the character of the particular frustule chosen : some easily show a good image, while others with any arrangement of illumination will with difficulty yield a good result.

Plate IX, Figs. 2, 3, 4, and 5.-Diatom : Navicula rhomboides. $\times 750$.

Objective, $\frac{1}{6}$-inch achromatic, by W. Watson \& Sons.

Ocular-projection, No. 2.

Illuminant, quartz mercury-vapour lamp.

Condenser, Leitz oil-immersion, N.A. 1·40.

Screen, acid-green and filter-yellow- $K$, giving perfectly monochromatic light, $\lambda 5461$.

The whole purpose of these four photographs is to show the effect of varying the incidence of the illumination. A slotted stop was placed at the back of the sub-stage condenser allowing only a small portion of the latter to be filled with light. The object was placed on a microscope with a rotating stage, and 
the photographs taken in varying positions so that the incidence of the light was changed in each case.

Fig. 2, the light was thrown in the direction of the length of the frustule, bringing out transverse lines.

Fig. 3, the light was in the direction of the width of the frustule, bringing out longitudinal lines.

Fig. 4, the incidence was between the two positions of Figs. 2 and 3 , bringing out the dotted structure.

Fig. 5 was taken with a central solid cone of light, but with reduced aperture.

Plate IX, Fig. 6.-Amphipleura pellucida. $\times 1500$.

Exactly the same conditions of illumination obtained as in Fig. 1, except that the sub-stage iris-diaphragm was rotated so that instead of the illumination being in the direction of the length of the frustule, it is approximately at an angle of $45^{\circ}$ to it. Very few frustules will show the dotted image at all, and it is only in exceptional specimens that it is possible to bring it out. The one chosen should be as large as possible, and as a rule it will be found that if the specimen is such that lines can be clearly shown in the direction of the length of the frustule by illuminating at right angles to the length, the dots may possibly be seen by suitably varying the incidence of the light. Perhaps the best method is to set up the object on a microscope with a rotating stage, arrange the illumination so that either transverse or longitudinal lines are shown, and then to rotate the stage carefully until the dotted image is seen with more or less distinctness.

Plate X, Fig. 1.-Surface of Metallic Alloy-Tin and Antimony. $\times 20$.

Three-inch achromatic objective, by James Swift \& Son.

No ocular.

Illuminant, electric arc, taking 4 amperes.

Convergent beam projected on to the specimen at an angle of $45^{\circ}$ by means of ordinary bull's-eye condenser.

Gifford F-line screen.

Plate, Imperial orthochromatic.

Exposure, 45 seconds.

Development by metol-hydrokinone. 
Prate X, Fig. 2.-Metal Surface-Silver and Copper Alloy. $\times 250$.

Objective, $\frac{1}{4}$-inch achromatic, by James Swift \& Son (corrected for uncovered objects). Tube-length, $220 \mathrm{~mm}$.

Zeiss projection-ocular, 2.

Mluminant, quartz mercury-vapour lamp, taking 2.5 amperes at 240 volts.

Zeiss vertical illuminator. Plano-convex lens interposed between the source of light and the illuminator, throwing a convergent beam into the reflecting prism of the latter.

Critical image of the illuminant projected on to the metal surface.

Screen, filter-yellow-K.

Wellington orthochromatic process plate.

Exposure, 20 seconds.

Development by pyro-soda.

Plate X, Fig. 3.-Cemented Steel, showing Carbide of Iron. $\times$ 100. (Negative by C. O. Bannister, Esq.)

Objective, 16-mm. apochromat, by Carl Zeiss.

Projection-ocular, No. 2.

Zeiss vertical illuminator.

Ordinary blue-green glass screen.

Camera-length, 80 centimetres.

Illuminant, electric arc, 20 amperes.

Plate, Barnet medium orthochromatic.

Fxposure, 16 seconds.

Plate X, Fig. 4.-Metal Surface-Manganese Sulphide in Steel. $\times$ 1000. (Negative by C. O. Bannister, Esq.)

Objective, 2-mm. apochromat; oil-immersion, N.A. 1.30 .

Projection-ocular, 2.

Camera-length, 100 centimetres.

Blue-green glass screen.

Illuminant, electric arc, 20 amperes.

Exposure, 20 seconds. 
Plate I.

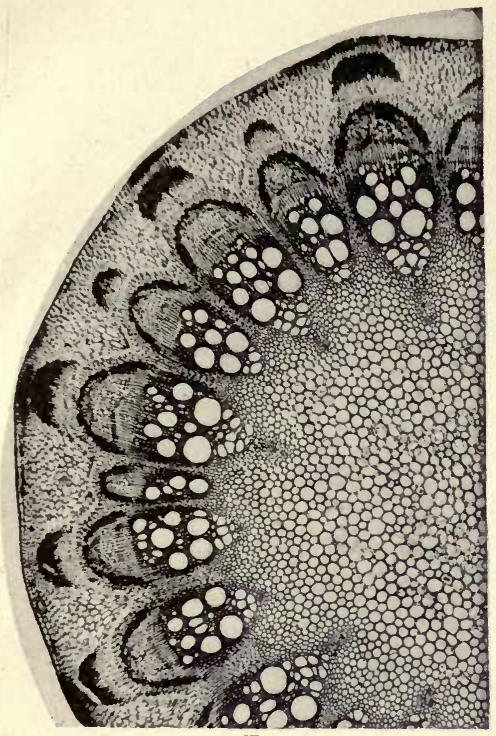

Fig I.

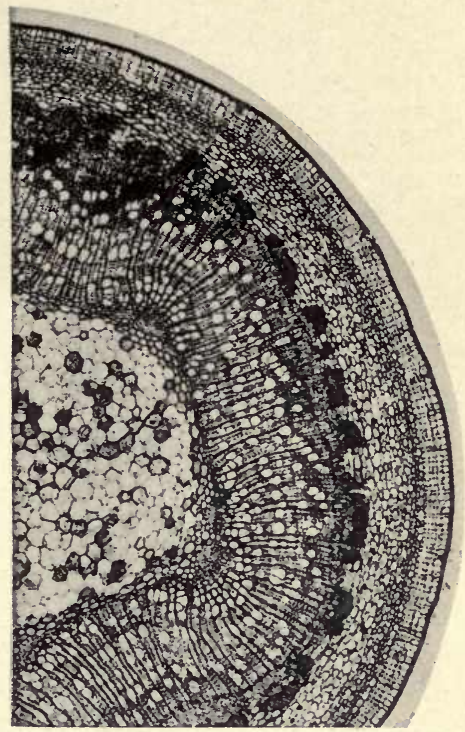

Fig 2.

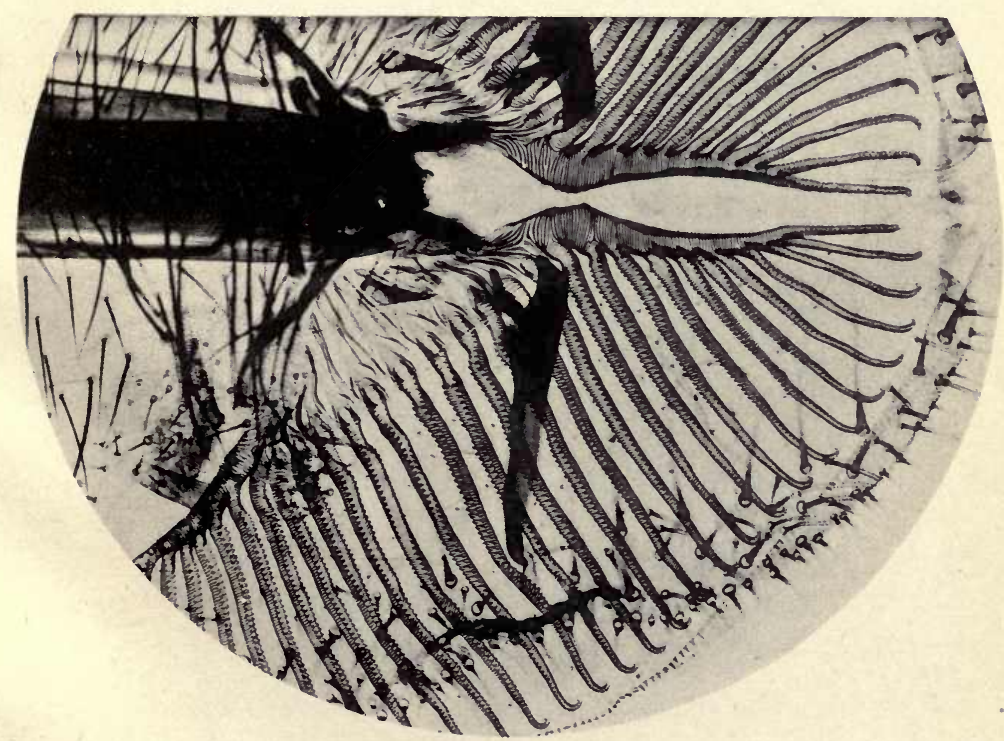

Fig 3. 

Plate II.

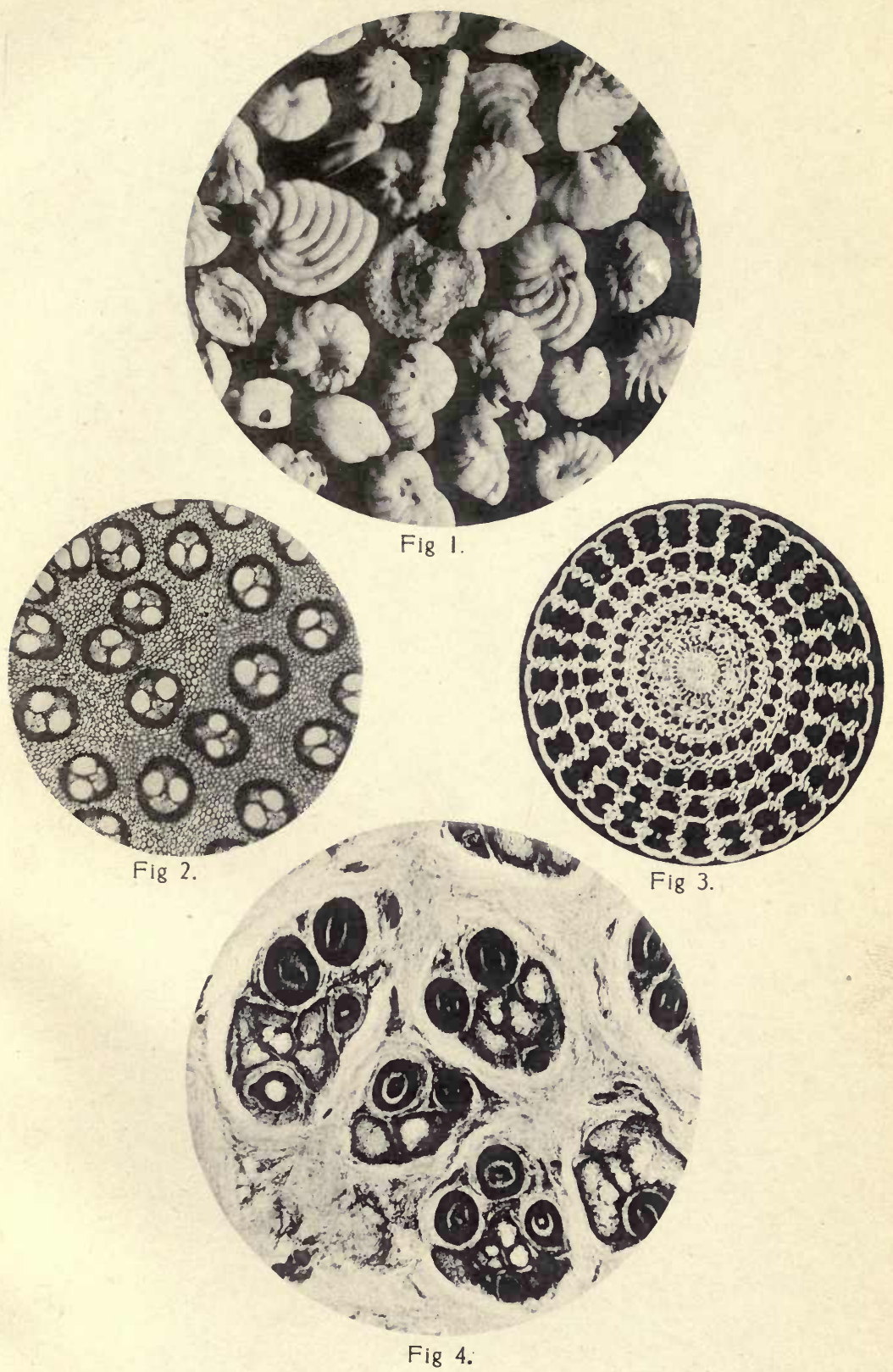



Plate III.

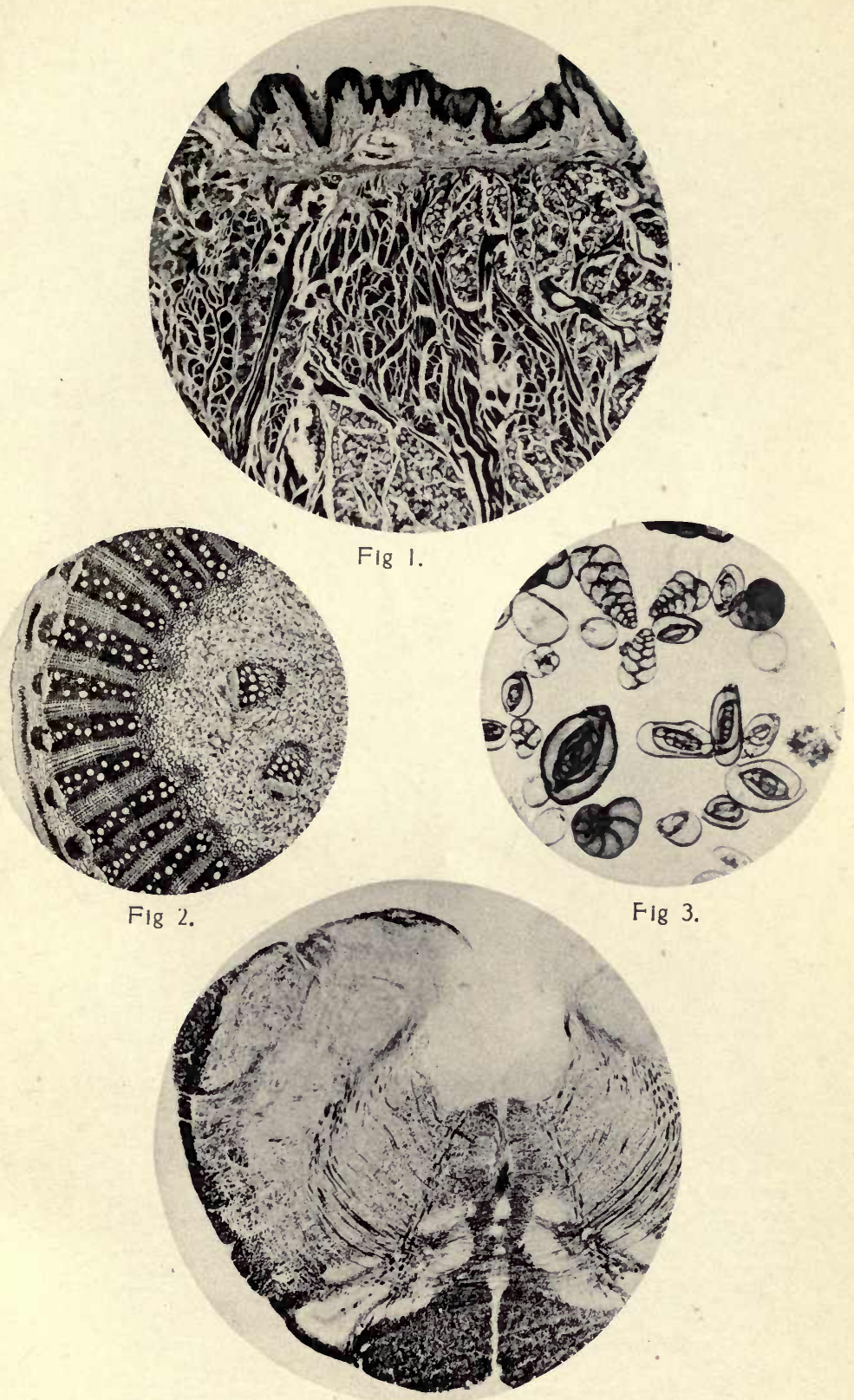

Fig 4. 

Plate IV.

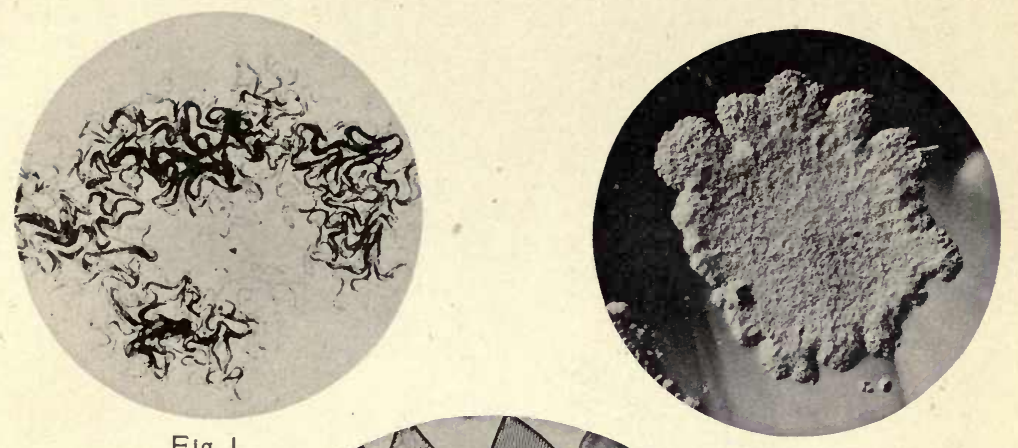

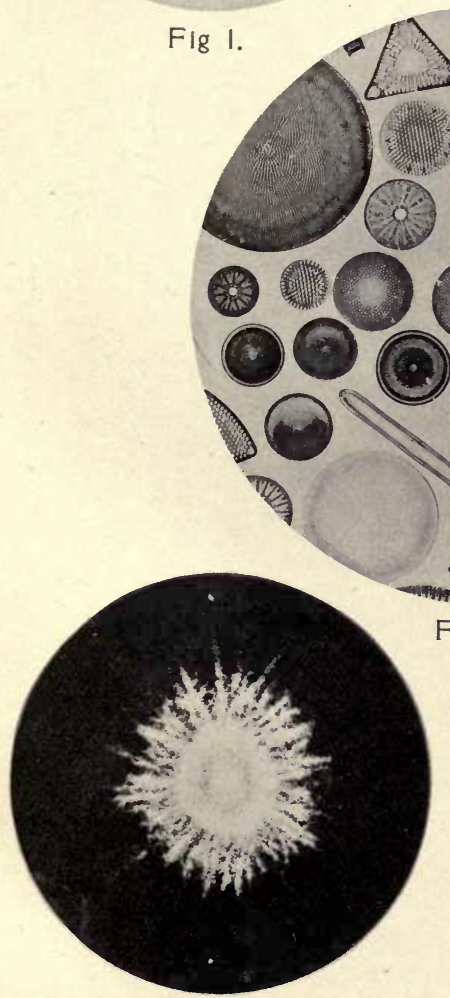

Fir 4.
Fig 2. 

Plate V.
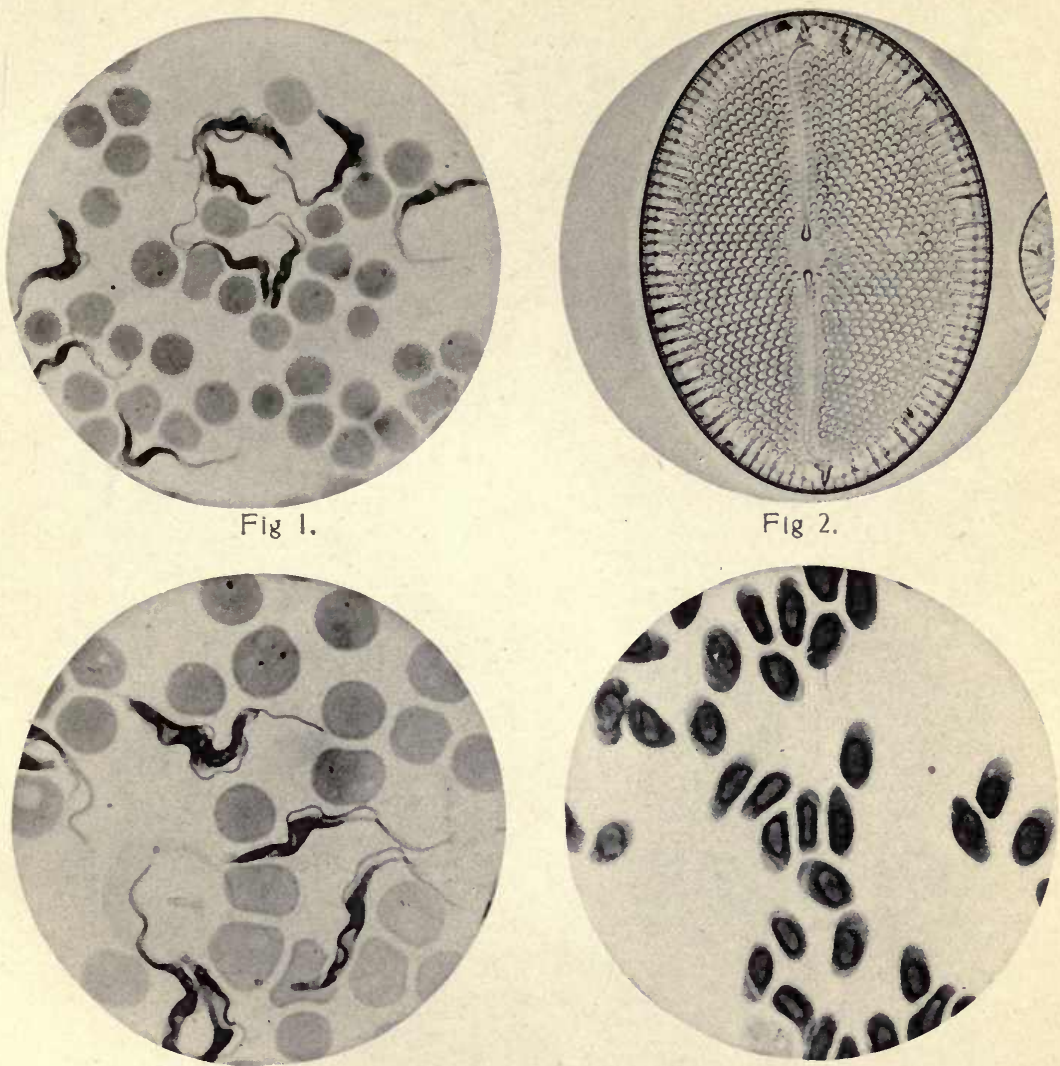

Fig 3.
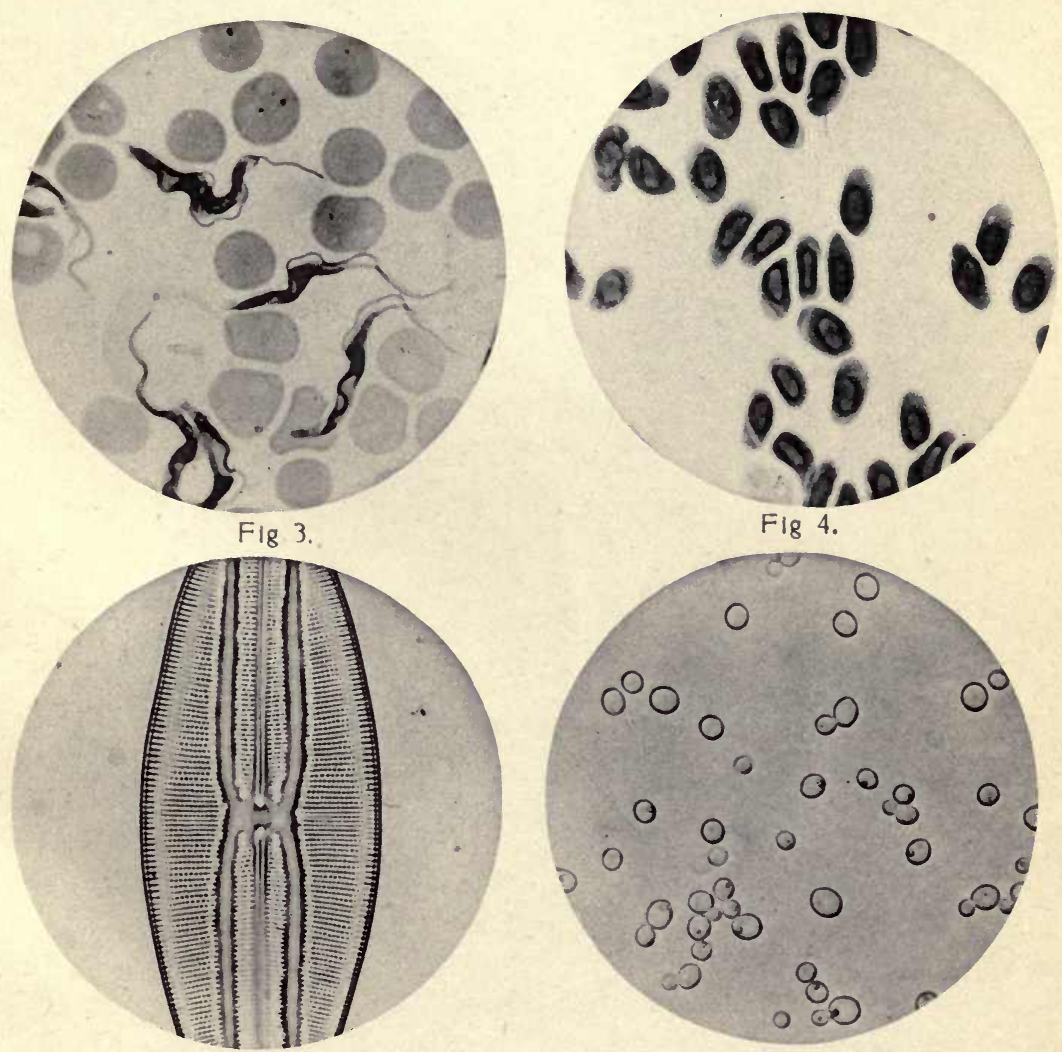

Fig 5.

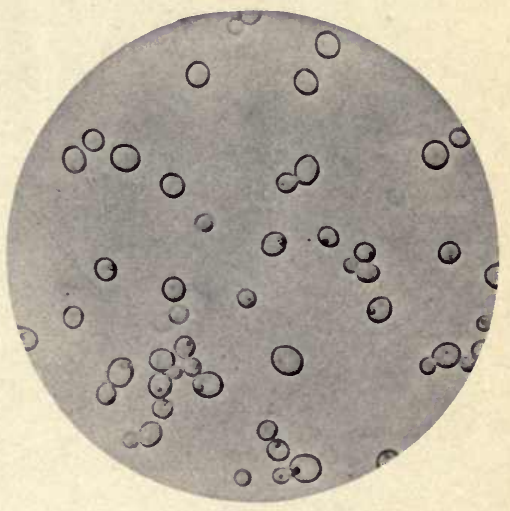

Fig 6. 



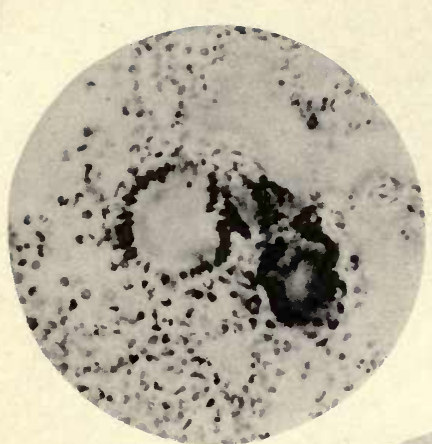

Fig 1.

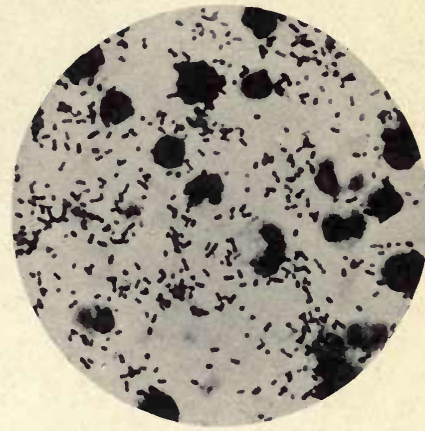

Fig 2.

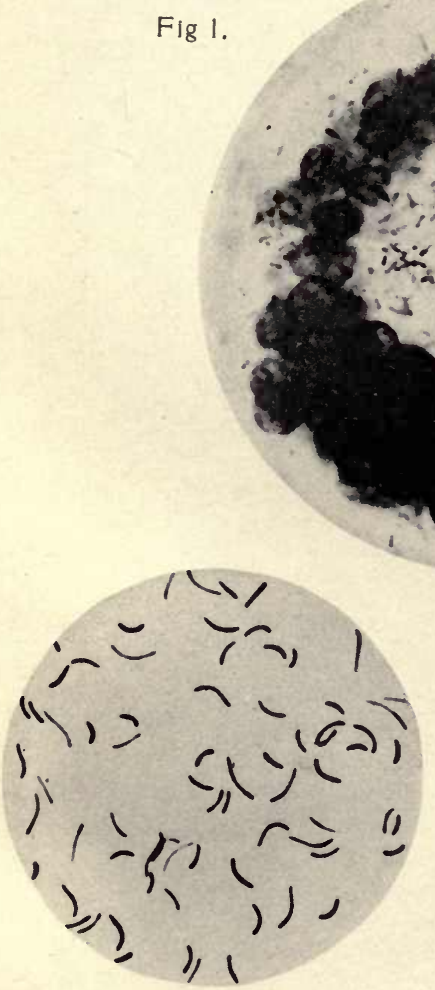

Fig 4.

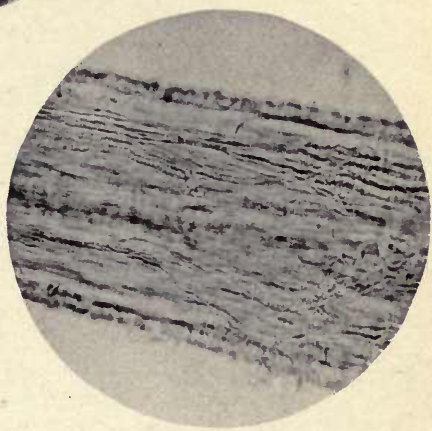

Fig 5. 



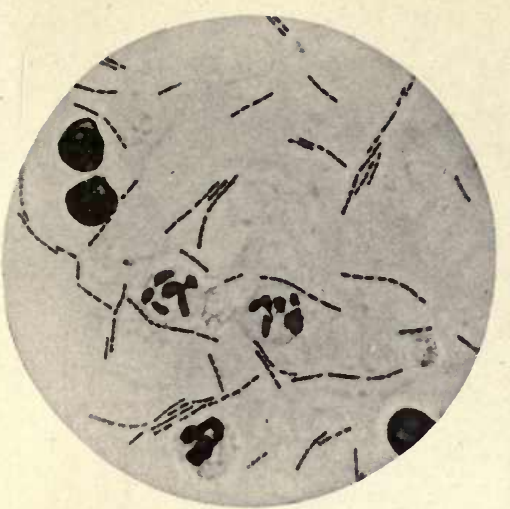

Fig 1.

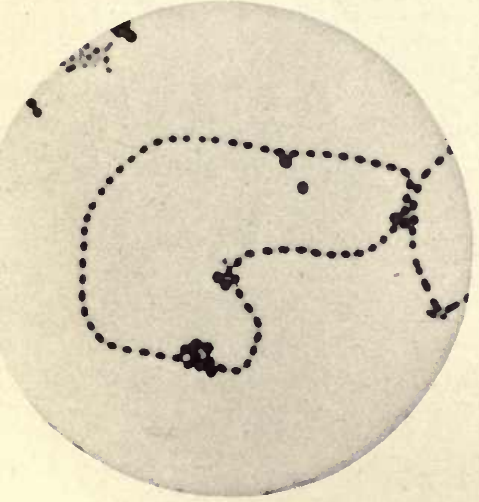

Fig 3.

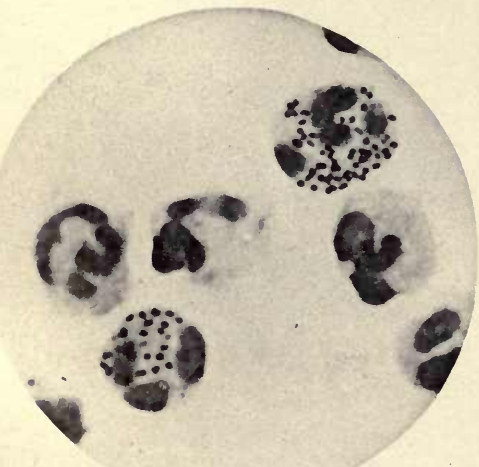

Fig 5.

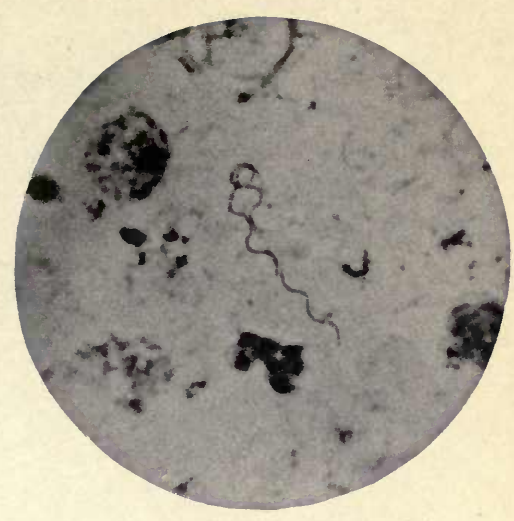

Fig 2.

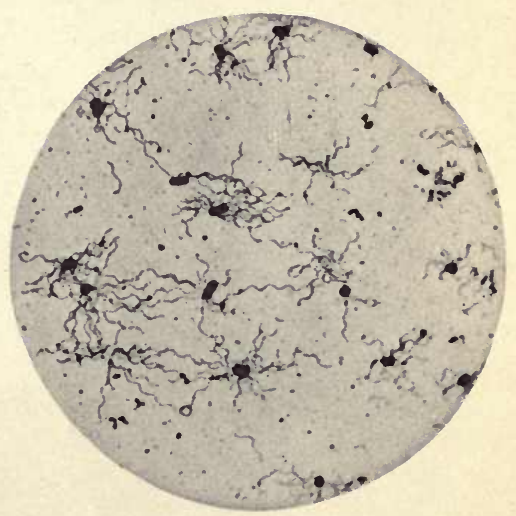

Fig 4.

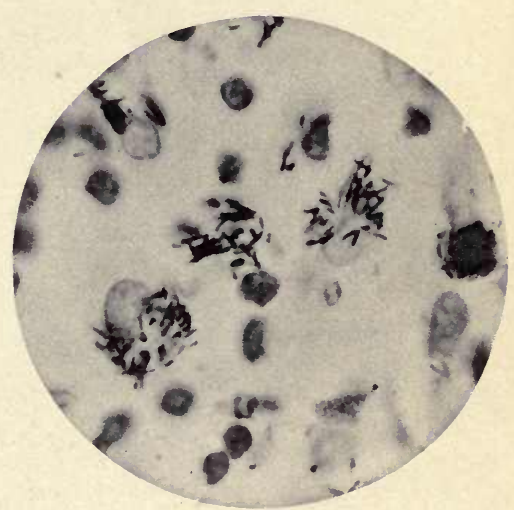

Fig 6. 

Plate VIII.

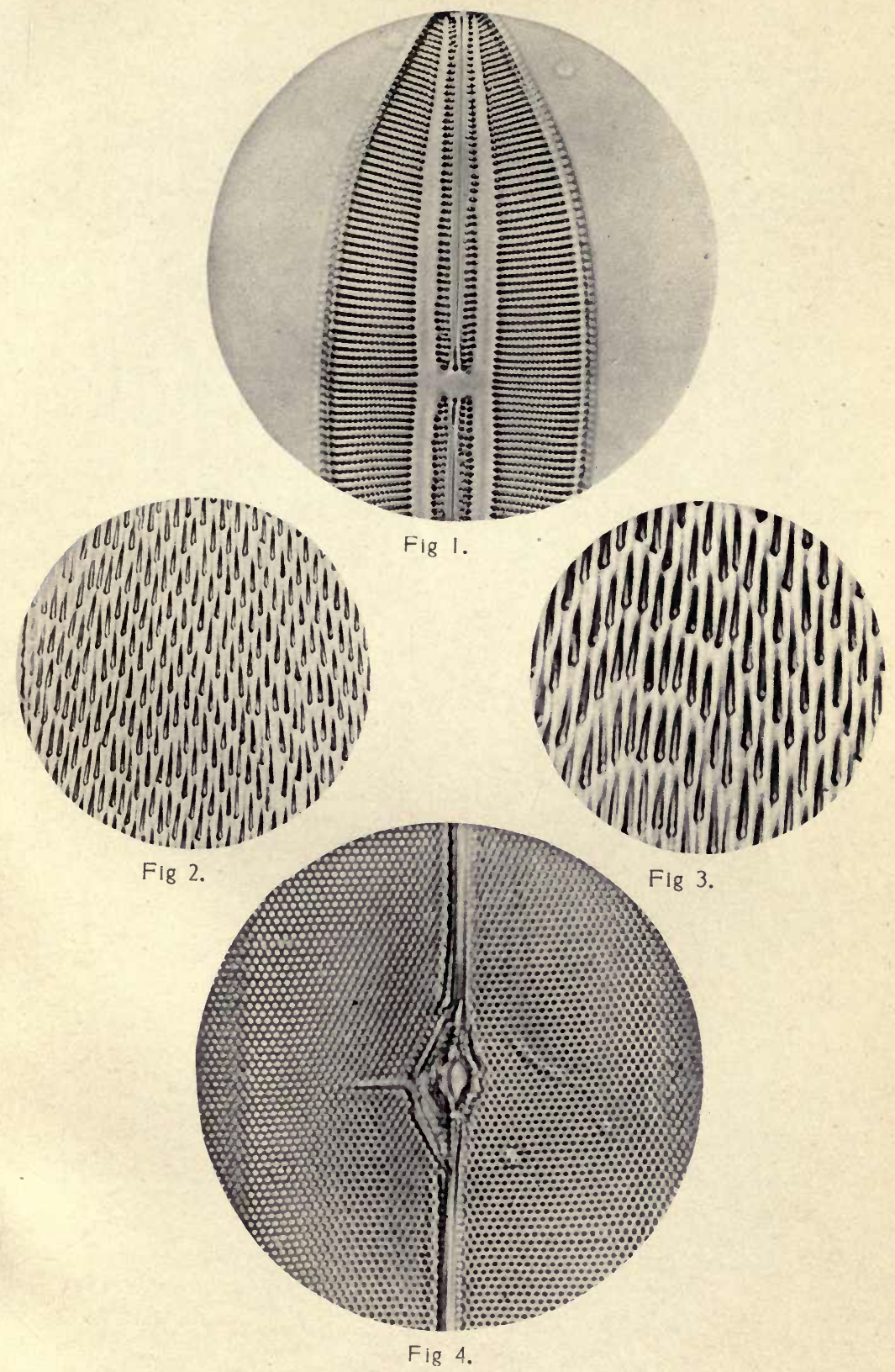





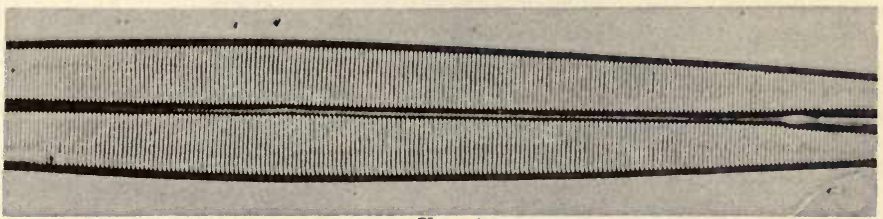

Fig I.

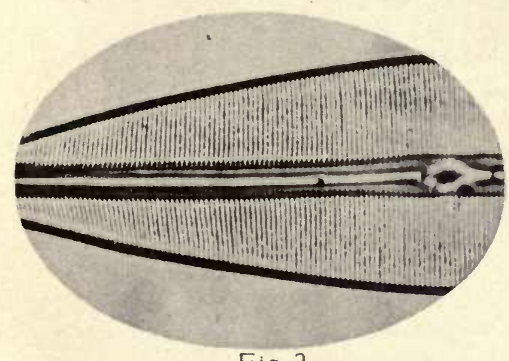

Fig 2.

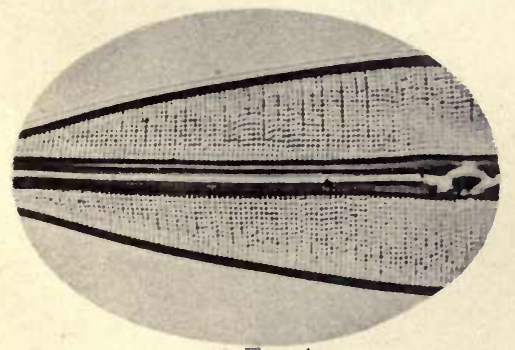

Fig 4.

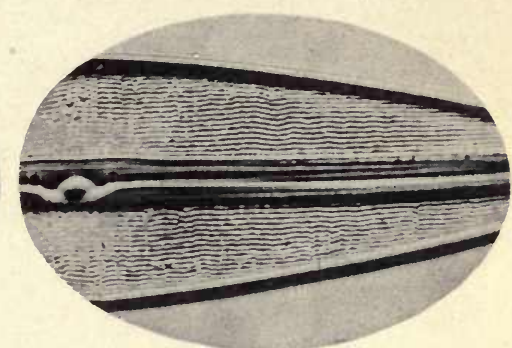

Fig 3.

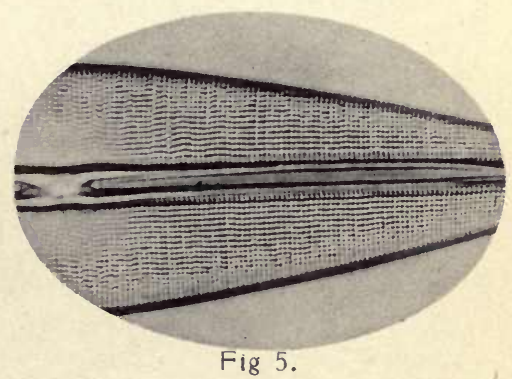

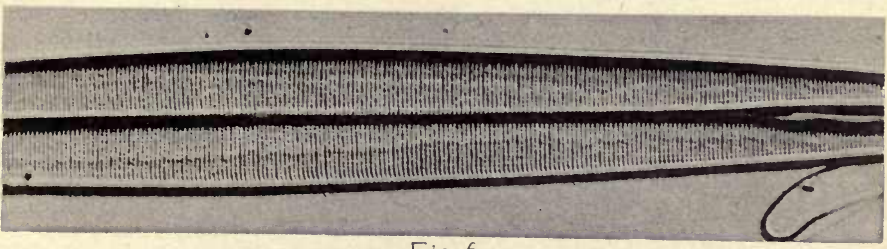

Fig 6. 

Plate X.

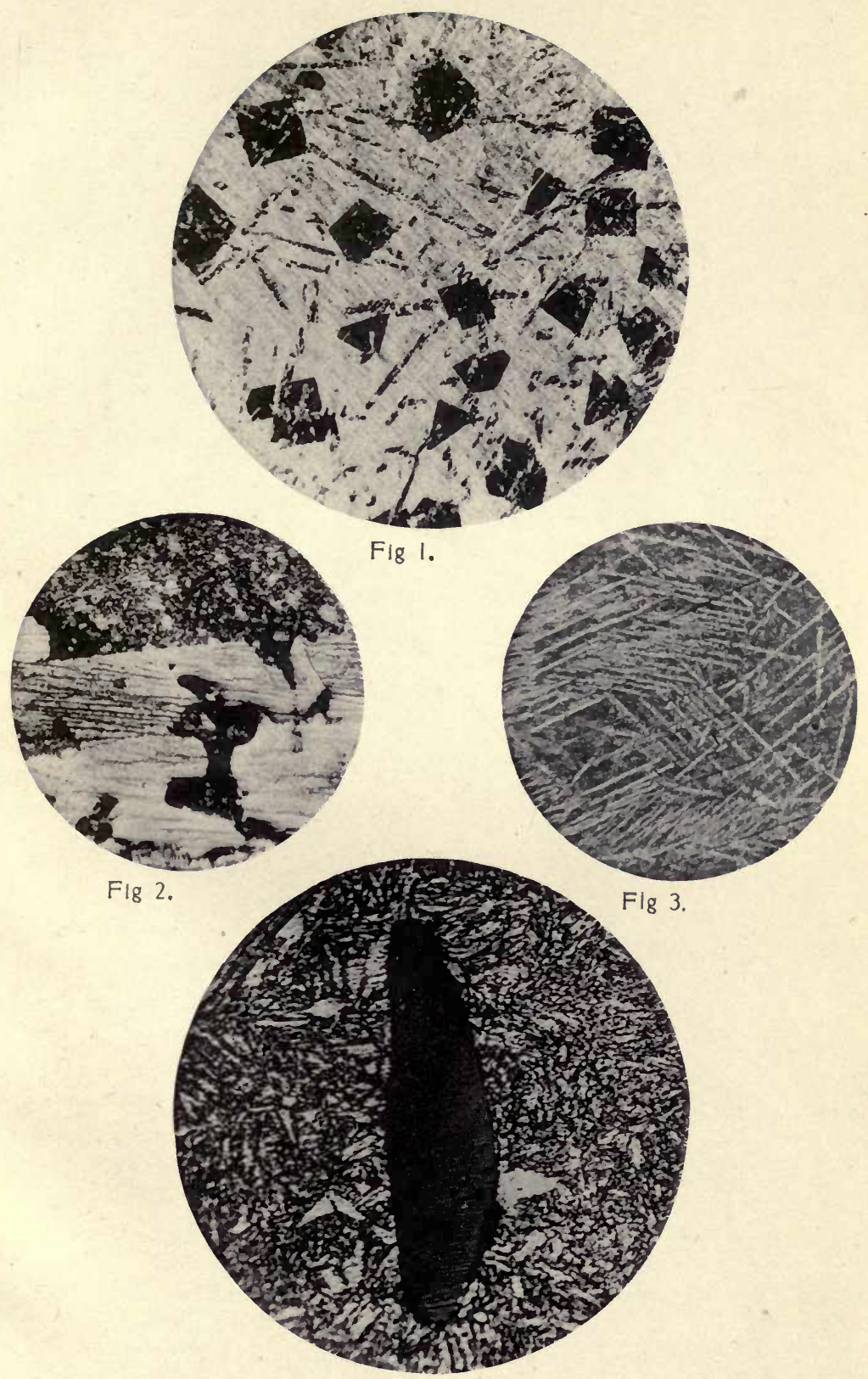

Fig 4. 



\section{APPENDICES}

\section{I.-Microscopic and Spectroscopic Standards of Measurements}

Micron $(1 \mu)=\frac{1}{1000}$ millimetre $\left(10^{-3} \mathrm{~mm}.\right)=0.000039$ inch. Micro-millimetre $(1 \mu \mu)=\frac{1}{1000}$ micron $=1$ millionth of a millimetre.

Tenth-metre $=0.1 \mu \mu=10^{-10}$ metre.

\section{II.-Weights and Measures}

The photographic formulæ have in most instances in this book been given in metric measures, but in certain cases for convenience and as a matter of custom the English standards have been used. The advantage of the metric system is so obvious as to need no recommendation here.

Metric Unit of Weight is the gramme $=15.4$ grains.

Decigramme

Centigramme

Milligramme

Kilogramme

Millimetre (mm.)

Centimetre (cm.)

Metre (m.)

Inch

Litre

Ounce $=\frac{1}{10}$ gramme $=0.1 \quad$ grm. $=1.54$ grains.

$=\frac{1}{100}$ gramme $=0.01 \mathrm{grm} .=0.154 \quad "$

$=\frac{1}{1000}$ gramme $=0.001$ grm. $=0.015$,

$=1000$ grammes $=1$ kilo.

$=\frac{1}{1000}$ metre $=0.03937$ inch.

$=\frac{1}{100}$ metre $=0.3937$

$=39.37$ inches.

$=25 \cdot 4$ millimetres.

$={ }_{10 \frac{1}{00}}$ litre $=16$ minims (approximate). 


\section{III.-REFractive INDICES}

Approximate refractive indices of some substances occurring and used in microscopic work, for the D (sodium) line.

\begin{tabular}{|c|c|c|}
\hline Air . & . & . $1 \cdot 0$ \\
\hline Water . & . & . $1 \cdot 334$ \\
\hline Alum (sat. sol.). & . & . $1 \cdot 358$ \\
\hline Absolute alcohol & & . 1.368 \\
\hline Fluor-spar . & . & . $1 \cdot 438$ \\
\hline Cedar-wood oil & . & . $1 \cdot 510$ \\
\hline Canada balsam . & . & $1 \cdot 540$ \\
\hline Styrax . . & . $\quad$. & . $1 \cdot 582$ \\
\hline Monobromide of $\mathrm{r}$ & hthaline & . $1 \cdot 657$ \\
\hline Realgar . . & . $\quad$. & . $2 \cdot 549$ \\
\hline Crown glass & . & - 1.530 (about) \\
\hline Flint glass & . & . 1.540 to $1 \cdot 710$ \\
\hline
\end{tabular}

IV.-The Position of a Photographic Lens in Relation to the Focussing-screen of the Camera when a Defintte Degree of Enlargement or Reduction of an OBject or Print is Required

The following short tables indicate the relative position of the lens and camera-screen in photographing certain microscopic objects. The measurements may be taken from the diaphragm of the lens, it being assumed that in nearly all cases a symmetrical combination will be used and not an ordinary single achromatic lens. Should a single lens be used, the distance may be estimated from the back surface of the lens.

\section{Number of Times of Enlargement. \\ 1 \\ $\begin{array}{llll}2 & 3 & 4 & 5\end{array}$ \\ 6}

Focus of Lens.

Distance in Inches of Lens from Focussing-screen.

3 inches.

$\begin{array}{llllll}6 & 9 & 12 & 15 & 18 & 21\end{array}$

4 ,

$8 \quad 12$

5 ,

6 , 
Number of Times of Reduction.

$\begin{array}{lllll}2 & 3 & 4 & 5 & 6\end{array}$

Fccus of Iens.

3 inches.

$4,$,

$5 \quad$,

6 ,

9 .,

Distance in Inches of Lens from Focussing-screcn.

$\begin{array}{rccccc}6 & 4 \frac{1}{2} & 4 & 3 \frac{3}{4} & 3 \frac{1}{2} & 3 \frac{3}{7} \\ 8 & 6 & 5 \frac{1}{2} & 4 \frac{4}{5} & 4 \frac{2}{3} & 4 \frac{4}{7} \\ 10 & 7 \frac{1}{2} & 6 \frac{2}{3} & 6 \frac{1}{4} & 6 & 5 \frac{5}{6} \\ 12 & 9 & 8 & 7 \frac{1}{2} & 7 \frac{1}{5} & 7 \\ 18 & 13 \frac{1}{2} & 12 & 11 \frac{1}{4} & 10 \frac{4}{5} & 10 \frac{1}{2}\end{array}$

\section{V.-The Determination of the N.A. of Microscope OBJECTIVES}

While an expert observer is often able to estimate approximately the N.A. of a microscope objective by direct observation of some known specimen, yet in ordinary practice some more exact method of determination is necessary. There are two appliances available, either of which is suitable for use with both dry- and oil-immersion lenses; one is known as the Abbe Apertometer, and the other as ('heshire's Apertometer. There are other methods in use which are only applicable to dry lenses.

The Abbe appliance (Fig. 78) consists of a segment of glass about three inches in diameter and half an inch thick, the segmental portion of which $c c$ is bevelled from above downwards and towards the semicircular edge to an angle of $45^{\circ}$. Near the middle of the straight edge a small disc of silvered glass $a$ is affixed, from the centre of which a sniall circle of the silvering has been removed. Two sliding metal plates $b b$ are so fitted on the semicircular edge that they can be moved round to any position. This edge of the plate is also graduated and marked with the full range of apertures up to $1 \cdot 4$, which represents practically the highest N.A. which is likely to be measured. To use the appliance the microscope is placed in a vertical position, and the glass plate is placed on the stage with the graduated surface uppermost. The objective, the N.A. of which is to be measured, is placed on the microscope and the hole in the centre of the small glass disc is carefully focussed. An eye-piece which has a large field should be placed in position, the general conditions under which the objective is to be used, such as the correct tubelength, \&c., being duly observed. 
With the apertometer an additional objective is supplied. The draw-tube of the microscope is now removed, and this objective is screwed into the bottom of the draw-tube, the eye-piece and the original lens being still allowed to remain in position. The draw-tube is now returned to the body of the microscope, and is focussed down until the images of the two metal plates which slide round over the indices can be seen, these being moved round until they are in the field of view. It is important to see when returning the bodytube to the microscope that no alteration in the position of the objective which is being measured takes place. It must still remain in the position to which it was adjusted, to show with an ocular, and without the additional objective on the

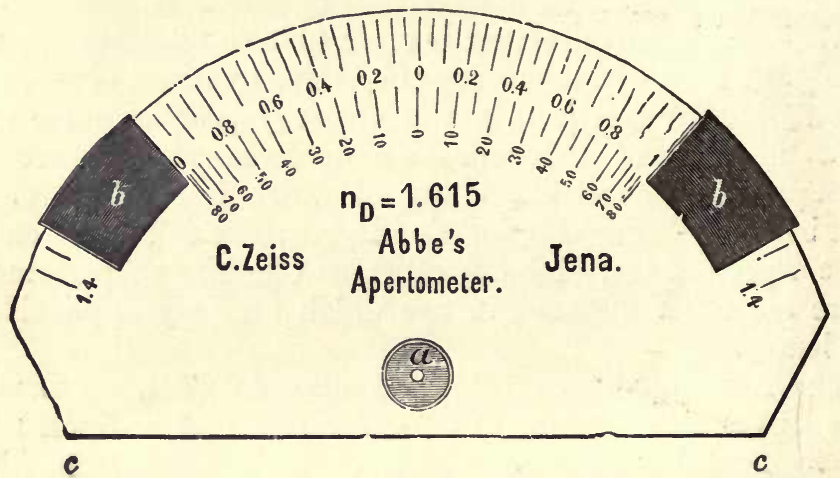

FiG. 78.-Apertometer, No. 155. (Full size.)

inside of the draw-tube, an image of the small spot in the centre of the silvered glass disc. The sliding metal pieces are carefully moved round until just their edges show on either side of the luminous disc as seen in the eye-piece. The reading of the indices may now be taken by direct observation, and if the objectives and eye-piece are all exactly central and in alignment it will be found that the reading is exactly the same on both sides, and that the reading represents the exact N.A. of the objective. It may happen that some slight difference in the reading on either side results, and in this case the mean of the two readings may be taken, the result given being the exact N.A. The observing ocular must have a sufficiently large field to enable the whole of the field of view to be directly observed.

The makers give the following further instructions :- 
- To obtain a suitable illumination for the use of the apertometer the light should be made to fall in a horizontal direction upon the cylindrical sides of the glass plate. In the event of the source of light not being wide enough to send sufficient light to both pointers, so as to cause them to appear brightly illuminated at the same time, the difficulty may be overcome by placing in front of the microscope a semi-circular translucent screen, e.g. an appropriately bent sheet of thin white paper. The upper edge of this screen should extend a little above the top of the apertometer.

- The power of the eye-piece used on the auxiliary microscope should rise with the magnifying power of the objective under examination, so as to obtain a sufficiently large image of the

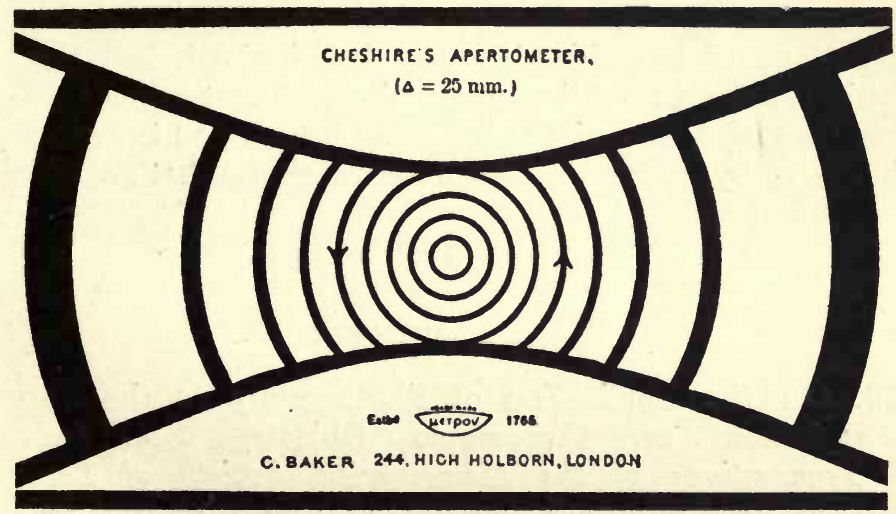

Fia. 79.

pointers, \&c., but on no account should an eye-piece be used the diaphragm of which partly cuts down the bright circle of light as seen through the auxiliary microscope.

'To measure with the apertometer low apertures (less than 0.4 or $50^{\circ}$ ), which are generally associated with long foci of $7 \mathrm{~mm}$. and more, the pointer, \&c. should be viewed with the unaided eye, without the auxiliary microscope. To this end in view the silver disc should be focussed, as described, and the eye-piece removed and replaced by a disc of cardboard or metal with a central hole. By this means the eye is kept in a central position whilst looking towards the objective.' 1

The apparatus is quite simple in use, and presents no unusual difficulty to a microscopist of even limited experience.

\footnotetext{
${ }^{1}$ See Directions for Use of Abbe Apertometer, by Carl Zeiss.
} 
The Cheshire apertometer (Fig. 79) is somewhat more simple in use, but it does not admit of such exact determinations with high apertures. It consists of a circular disc of glass with a mark on its upper surface, on which the objective to be measured is carefully focussed. The lower surface of the apertometer is ruled with a number of concentric rings, the distance between each corresponding to $0 \cdot 1$ N.A. In use the apertometer is placed on the stage of the microscope and the cross lines ruled on the surface are brought into focus, the eye-piece is then removed and by direct observation the number of concentric circles in view can be counted: this gives directly the N.A. of the objective.

With high powers it is difficult to sount the lines without some accessory observing appliance, since they appear so close together. A special eye-piece which focusses to the back focal plane of the objective may be obtained, and this is substituted for the ordinary eye-piece. The reading is then taken through the special eye-piece as before, and the aperture is indicated by the number of concentric circles that are visible.

\section{VI.-BibLIOGRAPHY}

1853. On the Application of Photography to the Representation of Microscopic Objects. J. Delves. Transactions of the Microscopical Society of London. (The first published account of any work on Photomicrography.)

BOOKS OF REFERENCE ON MICROSCOPY AND ALLIED SUBJECTS

Dr. W. B. CArpenter. - The Microscope and its Revelations. (Edited by the late Dr. Dallinger.)

Lewis Wright.-Popular Handbook to the Microscope.

W. Bagshaw.-Elementary Photo-micrography.

Conrad Beck.-Cantor Lectures on the Theory of the

Microscope. Royal Society of Arts. (One of the most lucid expositions of the subject yet written.)

Dr. E. C. Borsfield.-Guide to the Science of Photomicrography.

A. C. Maldey.-Micro-Photography. Wet Collodion Process.

A. Pringle.-Practical Photo-micrography.

F. Shillington Scales.-Practical Microscopy. 


\section{APPENDICES}

E. J. SpItTa.-Photo-micrography.

E. J. Spitta.-Microscopy.

Dr. H. van Heurck.-The Microscope. Translated by W. E. Baxter, F.G.S.

Sir A. E. Wright.-Principles of Microscopy.

\section{PERIODICALS}

Journal of the Royal Microscopical Society. Published every two months.

Journal of the Queckett Microscopical Club.

Quarterly Journal of Microscopical Science. 



\section{INDEX}

Авве, Prof., test-plate, 29, 32, 221 ; apochromatic objective, 40 ; condenser, 47,167 ; on advantage of powerful light, 76 ; law for resolution, 215 ; apertometer, 307

$\mathrm{Ab}$ rration, vide Chromatic, Spherical Absorption spectra of colour-screens, $205,210-218$; atlas of, 218

Acetate of copper, 212, 291, 294

Acetylene, 78, 169; lamps for, 79 ; generators for, 79 ; dissolved, 80 ; hent from, 79 ; actinic value of, 226

Acetylene Illuminating Co., 80

Achromatic, condensers, vide Substage condensers; objectives, vide Objectives

Acid-green, 213, 292, 302

Actinic values of illuminants, 78, 79, 226 ; comparison by exposure. meter, 225

Actinomycosis hovis, 295

Agar-solutions, 255, 268, 271

Allochrome plates, 288

'Alloys' by E. F. Laws, 265

Alloys, tin and antimony, 303, silver and copper, 304

Alum solution, 180 ; refractive index, 306

Amidol, 230, 240, 243, 288

Ammeter, 105

Amphipleura pellucida, 29, 301, 303

Anastigmat lens, 34

Angular aperture, 25

Angus, H. F. \& Co., 17, 292

Aniline dyes for colour-screens, 211214 ; with gelatin plates, 219

Annular illumination, 53, 167

Antipoints, 51

Apertometers, Abbe, 307 ; Cheshire, 309

Aplanatic cone, size of, 47-49, 53 ; advantage of large, 166, 167

Apochromatic, condensers, 50, 51, 300 ; objectives, vide Objectives
Arc light, 95 ; carbons for, 96-98; current-consumption, 99 : connections for, 104 ; protection of eyes from, 154; heat from, 180 ; actinic value of, 226

hand-feed, 99, 101, 102, Author's type, 101, Leitz, 106, 165, ad. justments of, 102, 103 ; automatic, Zeiss, 103, Leitz, 103

for instantaneous photo, 140 ; with sub-stage condenser, 168

Auramin, 289, 296, 298

Aurantia, 200, 211

Axial illumination, 28, 30

BAcrluUs, anthracis, 290, 291, 297 ; coli communis, 271, 290 ; of diphtheria (Krebs-Loffler), 271; filamentosus, 29; of leprosy, 299 ; pestis (Bubonic plague), 296; of tetanus, 271; tuberculosis, 29, 271, 296 : typhosus, 299

Backing of plates, 220, 221

Backlash, 6

Bacteria, tube (stab or streak) cultures, 268, 271, 272; plate-cultures with transparent or opaque colonies, 270 : gelatin-cultures, 268 , effect of temperature on, 272 ; apparatus for photographing, 267, 269, 273 ; illumination of, $57,117,139,268$, 271 ; water-bath to avoid reflection, 24,268 ; objectives for, 33,162 , as tests for objectives, 29: focussing of, 156, 191 ; instantaneous photos of, 139 ; to reproduce natural size, 273 ; pathological specimens, 273

Baker, C., microscope stand, 14; mercury-vapour lamp, 93, with quartz tubes, 94 ; Dr. E. J. Spitta's monochromatic apparatus, 215 ; circular stops for stereoscopic work, 275 ; Cheshire apertometer, 309 
Banfield, A. C., 275

Bannister, C. O., 304

Barnet orthochromatic plates, 220, 290, 293, 294, 298. 300

Bastian Mercury Lamp Co., quartz tubes, 94

Bausch \& Lomb, horizontal camera, 129

Beard, R., limelight regulator, 86

Beck, R. \& J., metallurgical microscope (Rosenhain), 260: rings for mounting specimens, 266 ; speculum for arc light, 108; vertical camera (Gordon), 119

Beck, Conrad. 310

Beech, stem of, 284

Beilby, G. T., 62

Bibliography, 310

Bichromate of potash, 212, 290

Blood, pigeon's, 294 ; guinea-pig, with $R$. anthracis, 297 ; in Bubonic plague, 296

Blow-fly, proboscis of, 28,285

Blow-through jet, vide lime-light

Botanical specimens, 162

Bousfield, Dr., 222, 310

British Oxygen Co., steel cylinders, 85

Bromide-prints, 199, 232, 239-240 ; developers for, 240

Bubonic plague, 296

Bull's-eye for illuminating opaque objects, 61,63 ; as auxiliary collecting-lens, 69,169 , how to use, 148 , 149

Burroughs Wellcome, tabloid developers, 234

Calcrum carbide, 78

Camera, position and support, 111, 117 , in relation to microscope, 112 , 188, on geometric slide, 133 ; connection to microscope, 178; precautions against diffused light, 179

vertical, Swift, 113, Zeiss, 114, 251, Leitz, 114, Van Heurck, 117, 295, J. W. Gordon (R. \& J. Beck), 119, 192, improvised form, 119

horizontal, Swift, 121, 129, Watson, 122, Ross, 123, Zeiss, 126, Bausch \& Lomb, 129, Author's. 130, 135, 175 ; control of fine adjustment, 122, Zeiss, $128,132,190$, author's, 130, 132 combined vertical and horizontal,
Leitz, 115, Watson, 116, Zeiss, 137

for instantaneous photos., 139; reflex, 140, 141, 295; for opaque objects, 141; for metallurgical objects, 141 , Leitz micrometallograph, 142

length of, with and without projection-ocular, 41, effect on magnification, 194, 195, 197, 283 ; size of, 181

Canada balsam, 24, 255; effect of mounting in, 154; refractive index, 306

Carbon-filament lamps, 88 ; difficulty from small area, 107, J. W. Gordon's device to overcome, 107

Carver, Dr. T. A. B., arc-lamp, 101 ; photo-micrographic apparatus, 130

Cedar-wood oil, 19, 150; for substage condensers, 56 ; refractive index, 306

Calls for fluid screens, vide Liquid cells

Cemented steel showing carbide of iron, 304

Centration of light, 148, 162, 164 ; of objectives, 32,163 , with nosepiece, 36-38 ; of sub-stage condensers, $161-166$

Centreing stage, 7 ; sub-stage of condensers, 8, 160

Chapman \& Jones, sensitometer, 223

Cheshire apertometer, 309

Cholera, Koch's spirillum of, 297

Chromatic aberration of objective, 21 ; tests for, $29-30,300$; correction for, 152

Clematis, stem of, 284

Collecting-lens systems, 69 ; object of, 162 ; advantages and dis. advantages, 169 ; method of using, 170-172, 174, with projected image, 173

bull's-eye, 61-63, 69, 169, how to use, 148, 149 ; E. M. Nelson's, $70,169,284,287$; WatsonConradv, 70, 169 ; Dr. Kohler's (Zeiss), 71, 290, how to use, 170172,181

supports for, 73, geometric-slide, 73, 170; with Welsbach gas, 74 ; with paraboloidal condenser, 58 ; as affecting working aperture of condenser, 173 ; in metallurgical work, 264

Collimator, 174

Collodion, films for colour-screen, 207; emulsion, 218 ; wet-plates, 218 , for 
lantern slides, 245; enamel for glazing. 242

Colonies of bacteria, 270, 271

Colour-contrast, 205 : to increase, 216 , 289. 292 ; to reduce, 217,288

Colour-fringes, 30 ; as test of ocular, 42

Colour-screens, uses of, 205 ; spectra of, 210-214

green, 210, 211, 292, 294, 302, Zettnow's. 212, for dark-room, 199 ; yellow, 180, 211, 227; yellow-green, 210, 212, 228, 288 ; blue, 213, 215

permanent, gelatin, 206, collodion, 207 ; fluid, cells for, improvised, 208, Zeiss, 208. Kingsford's trough, 209 ; glass, 210 , didymium, 218

as depending on illuminant, 213, 294, with mercury-vapour lamp. 92, 214; as affecting exposure, 227 ; to increase colour-contrast, 205, 216, 289, 292 , to reduce, 217 ; use with achromatic objectives, 153 ; when focussing, 191; with gelatin dry-plates, 219; in metallurgical work, 267; for coloured lantern-slides, 277, 280-282

Coloured lantern-slides, vide Lanternslides

Condensers, vide Sub-stage Condensers Cone of light. 23, 25; solid or hollow, 48 ; aplanatic, $47-49$ : size of, 51 , $162,166,167,292,295$

Continental model microscope, 7, 161

Continental tube-length, 40, 151

Copying diagrams, 270

Correction, for chromatic aberration, $21,29,30,300$; for spherical aber. ration, $21,29,30,47,48$; for coverglass thickness, 154-160

Correction-collar on objective, 33,155 ; adjustment of, 156-158

Cover-glass thickness, effect of, 154 ; correction for, by correction-collar, 56,155 , by alteration of tube-length, 155; use of Abhe test-plate, 29. of Podura scale, 158, of diatom, 159 ; measurement of, approximate, 156 , more exact, 157

D.P.H., microscope stand, 14

Dark-ground illumination, 28, 53. 56 ; by spot-lens, 53 ; by paraboloid, 54 ; by stop in sub-stage condenser, 54,287 ; by stop in objective, 56 ; by Zeiss paraboloidal condenser, 57 ; by Leitz dark-ground condenser, 59 ; J. W. Gordon's method, 59 ; resolving power with, 60 ; with vertical camera, 117 ; for instan. taneous photo. 140

Dark-room, equipment of, 201-204 ; illumination and lamps for, 198200 ; temperature of, 201, 230

Dark-slide, 183; in testing darkroom light, 201; in stereoscopic work, 274

Davis diaphragm, 286

Dalves, J.. 310

Depth of focl"a, vide Penetration

Desch, Dr. C. H., on metallography, 265

Developing, 229 ; factorial method,

1 230; solutions for, 229, 231,

particular cases, 287, 289. 292, 293. 295 ; for over-exposed plates, 232 , 233, for under-exposed, 233-235; of prints, 240 ; for lantern-slides, dry-plate, 243, wet-collodion, 247 ; for coloured lantern-slides, 278

Diaphragms between camera front and focussing-screen. 180 ; between $\mathrm{m}$ : croscope and light, 181

Diatoms, mounting of, 24 ; as tests for objectives, $28,29,159$; resolution of structure, $156,161,216$; illumination of, $60,293,295,302$, 303 : annular illumination of, 167 ; measurements of, 198 ; colourscreens for, 211; examples, 290, Amphipleura pellurida, 29, 301, 303, Navicula lyra, 294, 300, Orthonies splendida, 292, Pleurosigma angulatum, 28, 159, 301

Didymium glass, 218

Diffraction, fringes. 24, 51, 290, 293, 295,297 ; scattering of light, 222

Diffused light, 68,179

Dishes, porcelain, 203

Draining rack, 203

Dry lenses, vide Objectives, dry

Dry-plates, 218; for lantern-slides, 242-244; storage of, 204

Drving rack, 203

Dufay plate for coloured lantern-slides, $277,280-282$

Dust, on ocular, 153; shown by negative, 233

Echinds, spine of, 28. 287

Edward's " isochromatic plates, 220, 291, 292 ; Imperial, 287

Electric arc, vide Aro 
Electric illuminants, 88, vide Nernst, Mercury-vapour and Arc light, difficulty from small area of, 107, 169

English model microscope, 7

English tube-length, 40, 151

Enlargement, distance of lens from focussing-screen for, 306

Eosin, 214, 216, 294, 299

Ether vapour, substitute for coal gas, 81,84

Evenness of illumination, 69, 147, 149,162 ; importance of, 169

Exposing, method of, 189

Exposures, 222 ; by sensitometer, 222 ; based on working N.A. of objective, 223, Dr. D. J. Reid's method, 224; by exposure-meter (Wynne or Watkins), 225; as depending on illuminant, 77, 79, 226 , on speed of plate, 227 ; with colour-screens, 219, 227 ; under-, 227,233 ; over- , 227, 232, 233 ; in printing for lantern-slides, 242, 244,247 ; for coloured lanternslides, 277, 278 ; in metallography, 265 ; in bacteriological work, 272

Exposure-meters, Wynne or Watkins, 225

Eye-pieces, vide Oculars

Eyes, use of, to avoid fatigue, 147

Factorial method, 230

Filters for light, vide Colour-screens

Filter-yellow K screen, 211, 214, 289, $293,296,299,302,304$

Fixing, 229, 231; in lantern-slide making, 244

Flatness of field, 25 ; tests for, 29

Fluorite, 20, 51

Focal length of objective, 24, 26, 33, 50,193 , in stereoscopic work, 274; of condensers, 49 ; and magnification, 194, 195

Focus, depth of, vide Penetration

Focussing, preliminary visual trials, 146,150 ; with immersion objectives, 150 ; with achromatics for perfect definition, 152, 153; with apochromatics, 192; with reflex camera, 140, 141; with J. W. Gordon's apparatus, 192 ; with Leitz micro-metallograph, 143 ; control from a distance, 122, 128, $130,132,190$; method of, 190-192, with high-intensity illuminant, 154, 191, with ultra-violet light, 254; by projected image on opaque screen, 185, 186 ; eye-pieces for, 186, 187 ; spectacle lenses for 3187 ; of metal surfaces, 256 ; of bacteria, 156,191

Focussing eye-pieces, 186, Swift, 187 ; glass, binocular, 187

Focussing-screens, ground-glass, 183185, 191 ; plain-glass, 183, 184, 191 ; varnished-glass, 184 ; chemically made, 185 ; grain of, 183 , 185; insertion in groove, 183, distance from lens for given enlargement or reduction, 306

Foraminifera, 63, 285, 289

GeLatin films for colour-screens, 206 ; dry-plates, 218, 219; for lanternslides, 242

Geometric slide, principles of, 133, 136 ; for collecting-lenses, 73

Gifford F-line screen, 211, 227, 285, $289,290,299,300,303$

Glass, refractive index of, 157, 306.

Glass colour-screens, 210, 218, 288, 291,304

Glazing of prints, 241

Glycerin, 255, 268

Gordon, J. W., 43, 51 ; vertical camera, 119, focussing with, 192 ; method for dark-ground illumination, 59; speculum with lightsource of small area, 107

Grain of focussing-screen, 184, 185 ; of plates, $218,219,245$

Green aniline dyes, 211, 212

Green colour-screens, 288, 291-294, 300 ; for dark-room, 199, 200

Ground-glass screen, as illuminant, 169 ; for focussing, 183, 185, 191

Gwyer jet, 87

Halation, 220

Heat from illuminants, 70, 77, 79, 180

Heliostat, for sunlight, 108

Hetley, Messrs., glass for absorbing light, 191, 210

High-intensity illuminants, danger to eyes from, 154; difficulty from small area, 107, means of overcom. ing, 107, 108

High-power objectives, 19,28 ; ocular, 34

High-power work, collecting-lens system for, 72 ; focussing in, 150, 191 ; cover-glass thickness in, 154 Homogeneous system, 19, 35

Howard Farmer reducer, 236, 245 
ILFORD orthochromatio plates, 220

Illuminants, kinds of, 75 ; position of, 162, 164; vide Acetylene, Arc-light, Lime-light, Oil, Sunlight, Welsbach gas; heat from, 180 ; of high-intensity, danger to eyes from, 14 ; difficulty from small area, 107, means of overcoming, 107,108 ; for coloured lanternslide making, 278,280

Illumination, importance of, 69,75 ; vide Annular, Bacteria, Darkground, Metal Surfaces, Oblique, Opaque objects; of dark-room, 198-200

Immersion fluids, oil, 19; monobromide of naphthaline, 19; how to apply, 24, 150

Immersion lenses, vide Objectives, immersion

Imperial orthochromatic plates, 284 , 288, 296, 299, 301-303

Incandezcent gas, vide Welsbach gas

India-rubber supports for camera, 176

Instantaneous photos, 139, 141, 295

Intensification, 235, 298; in lanternslide making, 248, 249

Internal reflection, 152, 179, 287

Iris-diaphragm behind low-power objective, 22, 286; for use with collecting-lenses, $71,73,149,170-$ 174,181 ; under sub-stage condenser, 8, 49, use of, 148, 161 ; between microscope and light, 181 ; with vertical illuminator, 262

Isochromatic plates, $200,219,220,287$

Isolar plates, 220,221

KINGSFORD's trough, 209

Koch's spirillum of cholera, 297

Kohler, Dr., collecting-lens system, $71, \quad 170-172,181$; ultra-violet light apparatus, 250-255

LAMPS, vide Arc, Mercury-vapour, Oil ; for dark-room, 198--200

Lantern-slides, dry-plate, 232, 242 245 ; wet-collodion, $219,245-$ 248 ; illumination of darkroom, 199 ; projection of, 249

in colour, uses of, 276 ; Sanger Shepherd, three-colour method, 277-279; Lumière autochrome, Thames and Dufay colour-plate processes, 277, 280-282 ; development of negative, 278
Latherby, A., 224

Laws, E. F., on Alloys, 265

Leitz, E., arc lamps, automatic, 103, hand-feed, 106, 165 ; camera, vertical, 114, combined vertical and horizontal, 115 ; dark-ground condenser, 59; microscope stand, 14 ; micro-metallograph, 142 ; stops to reduce aperture of objec. tive, 22 ; vertical illuminator, 67 , in micro-metallograph, 144

Length of camera, vide Camera

Length of exposure, vide Exposure

Lenses, vide Objectives

Lepidocyrtus curvicollis, 158, 300, 301

Leprosy, bacillus of, 299

Leybold's cementing process, 208

Lieberkuhn reflector, 65

Light-excluders, 178

Light-filters, vide Colour-screens

Lime-light, actinic value of, 81,226 ; its production from coal-gas, 81 , from house-gas, 82 , from ether or gasoline, $81,82,84$

mixed jet, 81-83, limes for, 84, types of, 87, Gwyer, 87

blow-through, $82,84,86$, limes for, 85 ; Saturator method, 84, Pendant (Willway \& Sons), 84; Zirconium discs (Zeiss), 85

cylinders for, 85; regulator (Beard), 86 ; Pringle's cutoff tap, 87; stands for, 87; use of, 168, 169 ; heat from, 180

Limes, selection of, 84,85

Limit of visibility, 26, 60

Liquid-cells for dark-room, 199, 200 ; for colour-screens, 208, 209

Lister Institute of Preventive Medicine, 130,175

Low-power objectives, 19, 284 ; tests for, 28, 285

Low-power work, 24,286 ; collectinglens system for, 70, 73, focussing in, 150 ; light for, 76 ; with ground-glass screen as illuminant, 169 ; stereoscopic, 274

Lumière plates, 220; autochrome for coloured lantern-slides, 277 , 280-282

'Luna,' Nernst lamp, 90

Lung, B. tuberculosis in, 296

MACROPHOTOGRAPHY, 138

Magnesium light, 75 
Magnification, 193 ; of dry-lens, 19 ; of objectives and oculars, 26,34 , $35,193,194,197$; of oculars, 40 ; of projection-oculars, 41, 195; of object, to determine, 27, 198; calculation of, approximate, 195, by micrometer, 196; with ultraviolet light, 252; in metallurgical work, 264 ; in bacteriological work, 273

Manganese sulphide in steel, 304

Martens' metallurgical microscope, 259

Mees, Dr. Kenneth, Atlas of absorption-spectra, 218; on resolving power of plates, 221

Mercury-vapour lamp, 91 ; its spectrum, 92, 214 ; Baker's, 93, Swift, 94; connecting up, 94 ; quartz tubes for, 94, 154; method of using, 168, 181; colour-screens for, $92,214,218$

Metal-filament lamps, 88 ; difficulty from small area, IU7, J. W. Gordon's device to overcome, 107

Metal-surfaces, illumination of, 61$69,141,256,261,264,267$, vide also Upaque objects ; colour screens for, 267; microscopes, Rusenhain's (R. \& J. Beck) 259, Stead's (Swift) 263, Zeiss, 256, Martens' model (Zeiss) 259; Leitz Micrometallograpn, 142, 144; rotating. stage necessary, 8 ; coloured glass screens (Rosenhain), 261 ; exposure for, 264 ; focussing for, 256, 267 ; magnification for, 264; preparation and mounting of specimens, 24, 26b, 266 ; coloured lantern-slides for, 277

Metallography, improvements due to, 61 ; books on, 265 ; vide Metal Surfaces

Methyl green, 212

Methyl violet, 214, 298

Methylene-blue, 200, 213, 295-297, 299,302

Metol-hydrokinone, 230-232, 240, 242, $284,287,295$

Metric measures, 305

Micrometallograph (Leitz), 142, 144

Micrometer to measure magnification, 196,252 ; quart z, 255

Micromillimetre $(\mu \mu), \mathbf{5 0 5}$

Micron $(\mu), 305$

Microscope, preliminary adjustments of, 146 ; position relatively to camera, 112, 188; cunnection to camera, 178 .
Microscope stands, English and Con. tinental, 5; choice of, 6,9 ; desiderata in, 6-8; D.P.H. (Baker), 14 ; Leitz, 14; Reichert, 17 ; Research (Swift), 14; Royal (Watson), 14 ; Van Heurck (Watson), 11; Wales type (Swift), 9 ; Zeiss, 11, 256 ; Rosenhain's metallurgical (Beck), 259; Stead's metallurgical (Swift), 263; Martens' metalurgical (Zeiss), 259

Microsporon audouini, 297

Micro-Summar lens, 33, ¿84

Mirror as sub-stage condenser, 47

Mixed-jets, vide Lime-light

Monkey, medulla of, 289

Monobromide of naphthaline, 19 ; refractive index of, 306

Monochromatic light, 21, 27, 92, 302 ; from mercury-vapour lamp, 214; spectroscopic apparatus for (Baker), 214; filters (Wratten and Wain. wright), 217 ; resolution by, 216

Monochromatic objectives, 27, 251

Mounting of objects, 24; for ultraviolet light, 255 ; of metal speci. mens, 265, 266

NAPHTHOL-green, 212, 298

Navicula lyra, 294, 300 ; rhomboides, 302

Negative development, vide Developing

Nelson, E. M., collecting-lens system, $70,169,284$

Nernst lamps, 88-90, 169 ; actinic value of, 226 ; 'Luna' type, 90 , 165 ; Projector type, 91 ; connections for, 90 ; with Zeiss paraboloidal condenser, 58

Newman and Guardia, binocular focussing-glass, 187 ; reflex camera, 295

Nose-pieces, 34, 36, 37, 163

Numerical aperture of objective, 21, 25 ; importance of, 22,25 , 26,31 ; means of reducing, 22 ; in relation to resolving power, 21, 25, 26, 31, 161, Abbe's law, 215 ; as affecting penetration, 22-24, 194 ; proportion utilised, 162 ; as determining exposure, 223; Dr. D. J. Reid's method of measuring working value, 224; exact determination of, by Abbe apertometer, 307 , by Cheshire apertometer, 309 
of sub-stage condensers, 46,49 , 52 ; relation to aplanatic cone, 47, 53; means of reducing, 49,50 , to increase penetration, $22,296,299$, to give contrast, 297; effective working value, 52,167 , relative to N.A. of objective, 161 : in dark-ground illumination, 55 ,

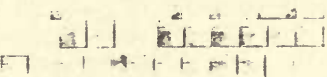

ObJective-ChaNgers, nose-piece, 34 , 36, 37, 163 ; sliding, 32, (Zeiss), 36, 151, 163; Watson \& Sons', 38; centration with, $32,36,38,163$; effect on tube-length, 37,151

Objectives, choice of, 18, 21, 26, 27 ; tests for, 28-32, 285 ; selected list, 33 ; comparison of types, 284

achromatic, meaning of, 18, 21 ; description of, 26,193 ; with projection-ocular, 40, 152, without ocular, 41 , with com. pensating ocular 42 ; correction-collar on, 155; colourscreens with, 205, vide Colour-screens; exact focussing for photography, 152, 153 ; magnifying power of, 35,193 ; tests for defining power, 28,300 , for correction, 29-32

apochromatic, meaning of, 18, 21 ; description of, 27, 195 ; Zeiss, 26, 30, 33, 284 ; with ordinary ocular, 40 , with projection-ocular, 40, 152, with compensating ocular, 42, 152 ; correction-collar on, 33,155 ; focussing with, 192; magnifying power of, 35,195 ; tests for defining power, 28, 29, for correction, 29-32

monochromats for ultra-violet light, 27, 251, 253

semi-apochromatic, 20 , with compensating ocular, 42

dry-lens, 19; advantage of, 156 ; tests for, 28-30; influence of cover-glass thickness, 160 ; for metallography, 263

immersion-lens, advantage of, 19 ; tests for, 29,30 ; focussing with, 150, correction for coverglass thickness, 156 ; in metal- lurgical work, 24 ; with verti. cal illuminators, 68

care of, 151 ; centration of, 32 , $36-38,163$; combination with ocular, 40-43, 152, 194 ; correction for chromatic and spherical aberration, 21, tests for, 29-30, 300 ; correction for cover-glass thickness, or tube length, vide Cover-glass thickness; focal length of, 26,193 ; flatness of field, 25 , tests for, 29 ; magnifying power of, $26,34,35,193$, vide also Magnification; numerical aperture, vide Numerical aperture; penetration of, 21-24, 286, vide also Penetration; relation to tube-length, 151 ; resolving power of, $26,28,60$, as depending on N.A., 31, vide also Resolving power; stopping down to increase penetration, 22-24, 59; for bacteriological work, 33, 162 ; for metallography, 263

Object-slide thickness, 58

Oblique illumination, to test objectives, 28,30 ; of bacteria, 268, 290 ; of diatoms, 302, 303; of metal surfaces, 61,63 ; of opaque objects, 285,286 ; by decentreing sub-stage iris, 302

Oculars, ordinary, 38, 42 ; compensating, $38,40,42$, 152 ; Huyghenian, 38, 40 ; Ramsden, 38, 40,186

with achromatic objectives, 35 , 38,42 ; with apochromatic objectives, 40, 152; with either achromatic or apochromatic, the Universal (Swift), 42, the Holoscopic (Watson), 42 ; projection, $32,38,40$, 41, 152, Zeiss, 44 ; quartz for ultra-violet light, 251

combination with objective, 34 , $41,42,152,194$; dust on, 153 ; focussing, vide Focussing eyepieces ; magnification of, 34 , $35,40,195$; selection of, 152

Oil, for immersion lenses and condensers, cedar-wood, 19; how to apply, 24, 56, 150 ; for lamps, 77

Oil-immersion condensers, 49, 55, 56 , Swift, 50, Leitz, 50 ; lenses, vide Objectives, immersion

Oil lamps, 76, 166, 169 ; heat from, 77 ; actinic value of, 226 
Opaque objects, illumination of, 61 ; by bull's-eye, 61,63 ; by parabolic side-reflector, 64 ; by Sorby parabolic reflector, 64 ; by Lieberkuhn reflector, 65 ; by reflecting glass for low power, 65

with oblique beam, $61-63,285$, 286 ; by vertical illuminator, 62, 68, 141, 256, Watsons', 66, Zeiss, 66, Leitz, 67, Rosenhain's, 68, 261, in Leitz micro-metallograph, 142-144 ; examples, 303, 304; vide Metal Surfaces

Opaque screen for centreing, \&c., vide Projection-image

Orthochromatic plates, 200, 220, 284

Orthonies splendida, 292

Ortol, developer, 230

Over-exposure, 232, 234

Oxyliydrogen, vide Lime-light

P.O.P., 238

Panchromatic plates, 199, 200, 219

Parabolic side-reflector, 64 ; Sorby, 64

Paraboloid condenser, 54, 57

Paraboloidal condenser (Zeiss). 57

Pathological specimens, 162, 273

Pendant Saturator (Willway and Sons), 84

Penetration, 1, 21; to obtain, by reducing aperture of sub-stage condenser, $22,296,299$, by reducing aperture of objective, 22,24 , 286 ; as affected by density of medium, 24, by focal length of objective, 24, by combination of objective and ocular, 43, 194 ; in low-power work, 24, 286; in stereoscopic work, 274

Pepper, stem of black, 288

Perchloride of iron, 180

Petroleum lamps, vide Oil lamps

Photo-micrography, its field, 3 ; how to begin, 4 ; progressive example of, $283 \mathrm{ff}$

Picric acid, 211, 284, 288

Pigg, J. I., 176

Planar lenses, 33, 181, 284, 286

Plates, vide Sensitive plates; for lantern-slides, 242; for coloured lantern-slides, 277, 280, 282

Plate-carriers, 181 ; tests for registration, 182

Plate-cultures of bacteria, 268

Pleurosigma angulatum, 29, 301; as test for objective, 159
Podura scale, as test of objective, 28,29 ; for correction for coverglass thickness, 159 ; examples, 300,301

Pringle, Andrew, lime-light cut-off tap, 87; microscope, 9 ; photomicrography apparatus, 129

Printing, 237; by daylight (P.0.P.), 238: by artificial light (bromide), 239.240 ; to glaze the print, 241 ; in lantern-slide making, 242, 244

Projection, preliminary experiments in, 147; of lantern-slides, 249

Projection-image on opaque screen, 154 ; use of, for centreing, 164, 185, 186 ; for studying collecting-lens effect, 173

Projection-oculars, vide Oculars, projection

Projector Nernst lamp, 91

Pyrogallic acid, 230, 240

QUARTz, tubes for mercury-vapour lamp, 94; in ultra-violet light apparatus, 251-253, 255

RAMSDEN, disc, 224

Realgar, 24 ; refractive index of, 306

Reducing, 236 ; in lantern-slide making, 245

Reduction, distance of lens from focussing-screen for, 306

Reflex camera, 140, 141, 295

Refraction, 19

Refractive index of mounting medium, 24, 156; of agar, 268 ; of cedar-wood oil, 19; of glass, 157, 306 ; table of, for various substances, 306

Reichert, C., microscope stand, 17 ; objective, 292

Reid, Dr. D. J., 224

Research microscope, 14

Resolving power, 26, Abbe's law, 215 ; dependent on N.A., 25, 31, on wave-length. 250 ; with darkground illumination, 60 ; as affected by condenser aperture, 161 ; by violet light, 215 , by ultra. violet light, 250, 251; of plates, 221

Rheinberg, J., 274

Ringworm, surface colony of, 291 ; in human hair, 297

Rockers, 201, 233

Rosenhain, W., vertical illuminator, 68 ; metallurgical microscope, 259 ; coloured glass screens, 261 
Ross, Messrs., horizontal camera, 123 Royal microscope, 14

Royal Microscopical Society's Journal, 26, 224, 311

Ruby lamp for dark-room, 198

SANGER, SHEPHERD \& Co., green colour screens, 199 ; process for coloured lantern-slides, 277-279

Sarsaparilla, stem of, 286

Saturator method for acetylene light, 84

Scalp, human, 287

Screen, yellow, to absorb heat, 180

Searcher eye-piece, 251, 254

Semi-apochromatic objectives, 20,42

Sensitive plates, gelatin dry, 218, 219 ; dry collodion emulsion, 218 ; wet collodion, 218, for lanternslides, 245, isochromatic, 200, 219 , 220, Edward's, 220, Lumière, 220, Isolar, 200, 221, Wratten and Wainwright's, 220; orthochromatic, 200, Wellington's, 220, Ilford. 220, Barnet, 220; panchromatic, 219, 220; backing of, 220 : degree of sensitiveness, 75 ; grain of, 218, 219, 245 ; size of, 181 ; for ultra-violet light, 222,225

Sensitometers, 222, 223

Siedentopf, ultramicroscopic method of, 59

Sliding objective-changers, 32 ; Zeiss, 36,151

Sodium sulphite, 240, 241, 243

Sorby parabolic reflector. 64

Spectacle lenses for focussing, 187

Spectra from acetylene lamp, 79 ; from mercury-vapour lamp, 92, 214; from Welsbach gas, 78; from colour-screens, 210-214

Spectroscope, 200, 210, 213, 216

Speculum with are light, 108

Spherical aberration of objective, $21,29,30$; of condenser, 48

Spirochcete pallida, 298

Spitta, Dr. E. J., monochromatic apparatus, 215 ; works by, 310

Spot lens, 53

Stab cultures of bacteria, 268

Stage of microscope, 7, 8

Staining of specimens, 216, 235, 286$288,292,294,295$

Stead, J. E., 265, 264

Steel-spring supports for camera, 177

Stereoscopic photo - micrography, three methods, 274, 275; illumination for, 276
Stoney, Dr. G. Johnston, heliostat 109

Stopping down of objective, 23, 59

Streak cultures of bacteria, 268

Streptococcus pyogenes, 298

Styrax, 24; refractive index of, 306

Sub-stage condensers, centreing, 8 , 160 ; chromatic, 46, 49, 167 ; achromatic, $49,52,161,166$, 168, Swift, 50, Leitz, 50 ; apochromatic, 50, 51, 300; dry, 49, 161; oil-immersion, $49,55,56,161$, Swift, 50, Leitz, 50; Mirror, 47; Abbe illuminator, 47, 167; para. boloidal (Zeiss), 57 ; objective used as condenser, 50 ; Leitz, dark-ground, 59; Watson \& Sons'. 53 ; Zeiss centreingcollar, 50

aplanatic cone of, 47,53 , advantage of large, 166, 167; central stop in, $49,54,55,168$; centreing of, 161, 163-166 ; numerical aperture of, 46, 47, 52,161 ; reduction of a perture, $22,49,51,296,297,299$; effective working aperture, 52, 167 , as affected by auxiliary collecting-lens, 173 ; for darkground illumination, 53-55

Sunlight, 108; Stoney's heliostat, 109, substitute for, 109

Swift, J. \& Son, cameras, vertical,

113, horizontal, 121, large photomicrographic, 129 ; condenser, 50 ; focussing eye-piece, 187 ; mercuryvapour lamp, 94; microscope, Wales, 9, Research, 14; nose. piece, 36 ; ocular, Universal, 42

TABLOID, Burroughs Wellcome, developer, 234

Tartrazine, 211, 213, 289, 292, 293, 298

Taverner, H., 275

Temperature, changes of, 175

Thames colour plates, 277, 280-282

Thickness of cover-glass, vide Coverglass ; of object-slide, 58

Thomas's lantern-plate, 286

Thorn \& Hoddle Co., acetylene generator, 80

Tongue, cow's, 295 ; human. 288

Toning, 238; in lantern-slide making, 248

Tripanosoma gambiense, 292, 293 
Tube-length, 7 ; English and Continental, 40, 151. 195; adjustment to objective, $15 \%$, or objectivechanger, 36, 151 ; to correct for thickness of cover-glass, 155, 157160

\section{UNDER-EXPOSURE, 233-235}

Ultramicroscopic method, 59

Ultra-violet light. 250; resolving power in relation to wave-length, 250 ; monochromat, 251 : ZeissKohler apparatus for, 27, 250-253 ; how to use, 251-255; precautions with, 152 ; magnification by, 252 ; focussing with, 254 ; plates for, 222,255 ; preparation of specimens, 252

Uranium intensification, 235, 236

VAN HeURck, microscope stand, 11 ; camera, vertical, 117, 140, 295 ; 'The Microscope,' 311

Ventilation of dark-room, 202

Vernier, 7

Vertical illuminators and illumination, 61; vide Metal surfaces and Opaque objects

Vibration, 112, 175, 178; as depending on position of room, 175,176 , on foundations, 175 ; prevention by india-rubber supports, 176, by springs, 177, by floating, 178; when exposing, 189

Violet light, 214, 222 ; resolution by, 216

Voltmeter, 105

Von Rohr, Dr., 27

W ALES microscope, 9

Warnerke sensitometer, 222

Water-bath to absorb heat, 180 ; to stop reflection in bacteriological work, 24, 268

Water-supply of dark-room, 201

Water-vapour, to remove from bacteria tube, 272

Watkins, exposure-meter, 225

Watson, W. \& Sons, camera, horizontal, 122, combined vertical and horizontal, 116, vertical, 117, 140 ; heliostat, 108; holoscopic ocular, 42 ; Kingsford's trough, 209; microscope stand, Van Heurck, 11, Royal, 14; objective-changer,
38 ; sub-stage condensers, 53 ; vertical illuminator, 66

Watson-Conrady, collecting-lens sys. tem, 70, 169

Wave-length, 200, 212, 213, 216, 302 ; ult ra-violet, 250-252

Wellington orthochromatic plates, $220,285,289$ et ser.

Welsbach gas, 77 ; collecting-lenses for, 74; with Zeiss paraboloidal condensers, 58

Wet-collodion plates, 218 ; for lanternslides, 245

Willway \& Sons, Pendant saturator, 84

Wratten and Wainwright's green colour-screens, 199; monochromatic filters, 217; plates for lantern-slides, 242, 290, 301, Allo. chrome, 288, Panchromatic, 220 ; speed-value of plates. 227

Wynne exposure-meter, 225

Yeast CELLS, unstained living, 295

ZEISS, CARL, Abbe apertometer, 307 ; arc lamp, 103 ; camera, photomicrographic, 126, 141, combined vertical and horizontal, 137, horizontal, 126, vertical, 114 ; collecting-lens system (Kohler), 71, how to use, 170-172, 181 ; condensers, 50 , stop for, 55, paraboloidal, 57, centreing-collar for objective used as condenser, 50 ; control of fine adjustment, 128, 132, 190 ; filter blue-glass, 213 ; liquid cells, 208 ; microscope stand, 11, for metallurgical work, 256, Martens' model, 259 ; objectives, apochromatic, 26 , 30,33 , monochromatic, 27,251 , 253, of high aperture, 19, Planar, 276, Prolar, 273 ; oculars, compensating, 38, 40, projection, 44, tape measure for use with, 45; sliding objective-changer, 36,151 , 163 ; spherical flask, as collectinglens, 58 ; stops to reduce aperture of objective, 22 ; ultra-violet light apparatus, 250-255; vertical illuminator, 66 ; zirconium discs, 85 Zettnow's colour-filter, 212, 294, 300

Zirconium discs (Zeiss), 85

Zylol, 255 




\section{From}

\section{Mr. Edward Arnold's List}

of Medical Books.

\section{TEXT-BOOK OF

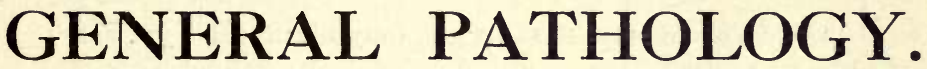

By the following contributors: A. P. BEDdARD, M.A., M.D., London; A. E. Boycotт, M.A., M.D., Manchester; C. H. Browning, M.D., Glasgow ; A. E. Garrod, M.A., M.D., F.R.S., London; J. S. Haldane, M.A., M.D., F.R.S., Oxford; I. WalkeR Hall, M.D., Bristol; A. F. Hertz, M.A., M.D., London; F. W. Motr, M.D., F.R.S., London; M. S. Pembrey, M.A., M.D., London; J. Ritchie, M.A., M.D., Edinburgh; S. V. Sewell, M.D., Melbourne; J. Lorrain Smith, M.A., M.D., F.R.S., Edinburgh ; E. Ainley Walker, M.A., M.D., Oxford.

Edited by M. S. Pembrey and J. Ritchie. Illustrated. One Volume. xii +773 pages. Demy 8vo. I8s. net.

\section{THE PRACTICE OF SURGERY.}

By Russell Howard, M.B., M.S. (Lond.), F.R.C.S. (Eng.), Surgeon to the Poplar Hospital; Assistant Surgeon to the London Hospital, etc. With 8 Coloured Plates and 523 Illustrations in the Text. viii +1227 pages. Royal 8vo. 2 Is. net.

The author of this new text-book on surgery has written especially for the student who is preparing for one of the qualifying examinations. At the same time it is hoped that the book may be useful to him after leaving hospital work, special attention having been given to a clear description of the treatment of diseases and modern methods of diagnosis. A special feature of the book is the concise descriptions of the clinical features of the various diseases of surgery, so that cases that have been seen in the wards are recalled to the memory. Directions for the after-treatment of operations have been given, and considerable space has been devoted to diseases of the nose, ear, pharynx, and larynx.

The text is fully illustrated by diagrams, photographs, and illustrations specially prepared for the book. 


\section{A NEW AND IMPORTANT WORK}

\section{COVERING THE ENTIRE FIELD OF -THE SUBJECT} BY LEADING AUTHORITIES.

\section{DISEASES OF CHILDREN.}

By VARIOUS AUTHORS.

Edited by Archibald E. Garrod, I.M., M.A., F.R.C.P., F.R.S.; Frederick E. BATTEN, M.A. (Camb.), M.D., F.R.C.P.; and Hugh Thursfield, D.M., M.A., F.R.C.P.

In One Volume, xviii + II84 pages, fully Illustrated. 3os. net.

\section{LIST OF AUTHORS:}

H. G. ADAMSON, M.D. (Lond.), F.R.C.P., Physician-in Charge Skin Department, St. Bartholomew's Hospital.

FREDERICK E. BATTEN, M.A. (Camb.), M.D., F.R.C.P., Physician to Hospital for Sick Children, Great Ormond Street.

J. W. CARR, M.D. (Lond.), F.R.C.P., F.R.C.S., Senior Physician, Victoria Hospital for Children, Chelsea.

EDMUND CAUTLEY, M.D. (Cantab.), F.R.C.P., Senior Physician, Belgrave Hospital for Children, London.

H. MORLEY FLETCHER, M.D. (Cantab.), F.R.C.P., Physician-in-Charge Children's Department, St. Bartholomew's Hospital.

J. S. FOWLER, M.D. (Edin.), F.R.C.P. (Edin.), Physician, Royal Hospital for Sick Children, Edinburgh.

ARCHIBALD E. GARROD, D.M. (Oxon.), M.A., F.R.C.P., F.R.S., Physician to the Hospital for Sick Children, Great Ormond Street, London.

E. W. GOODALL, M.D. (Lond.), Medical Superintendent, Eastern Hospital, London.

A. M. GOSSAGE, D.M. (Oxon.), F.R.C.P., Physician to Out-Patients, Westminster Hospital.

LEONARD G. GUTHRIE, D.M. (Oxon.), F.R.C.P., Physician, Paddington Green Children's Hospital.

ROBERT HUTCHISON, M.D. (Edin.), F.R.C.P., Physician to the London Hospital.

FREDERICK LANGMEAD, M.D. (Lond.), M.R.C.P., Assistant Physician, Hospital for Sick Children, Great Ormond Street.

F. J. POYNTON, M.D. (Lond.), F.R.C.P., Physician-in-Charge Children's Department, University College Hospital.

H. D. ROLLESTON, M.D. (Cantab.), F.R.C.P., Senior Physician, St. George's Hospital, London.

F. A. ROSE, M.B., B.C. (Cantab.), F.R.C.S., Assistant Surgeon, Throat Department, St. Bartholomew's Hospital.

GEORGE FRED. STILL, M.D. (Cantab.), F.R.C.P., Physician Children's Department, King's College Hospital.

G. A. SUTHERLAND, M.D. (Edin.), F.R.C.P., Physician, Paddington Green Children's Hospital.

H. THEODORE THOMPSON, M.D. (Cantab.), F.R.C.P., F.R.C.S., Assis. tant Physician to the London Hospital.

JOHN THOMSON, M.D. (Edin.), F.R.C.P. (Edin.), Physician to the Royal Hospital for Sick Children, Edinburgh.

HUGH THURSFIELD, D.M. (Oxon.), F.R.C.P., Physician to Out-Patients, Hospital for Sick Children, Great Ormond Street.

A. F. VOELCKER, M.D. (Lond.), F.R.C.P.; Physician, Hospital for Sick Children, Great Ormond Street.

G. E. WAUGH, M.D. (Lond.), B.S., F.R.C.S., Surgeon in-Charge Aural Departunent, Hospital for Sick Children, Great Ormond Street. 


\section{A MANUAL OF GYNACOLOGY}

\section{FOR STUDENTS AND PRACTITIONERS.}

By Samuel J. Cameron, Assistant to the Regius Professor of Midwifery, University of Glasgow; Gynæcologist and Assistant Obstetric Surgeon to the Royal Glasgow Maternity and Women's Hospital ; Gynæcologist to Out-patients, Glasgow Western Infirmary. Fully Illustrated, xvi $+55^{2}$ pages. I 8 s. net.

\section{THE BRAIN IN HEALTH AND DISEASE.}

By Joseph Shaw Bolton, M.D., D.Sc. (Lond.), F.R.C.P. (Lond.), Fellow of University College, London; Professor of Mental Diseases, University of Leeds; Medical Director West Riding Asylum, Wakefield. Illustrated. xiv +479 pages. I8s. net.

\section{ULCER OF THE STOMACH}

By Charles Bolton, M.D., D.Sc. (Lond.), F.R.C.P. (Lond.); Director of Pathological Studies and Research, and Lecturer in General Pathology, University College Hospital Medical School ; Physician (with Charge of Out-Patients) University College Hospital. With 35 full-page Plates and I 5 Figures in the Text. $\mathrm{xv}+396$ pages. 15 s. net.

\section{DISEASES OF THE HEART.}

By John Cowan, D.Sc., M.D., F.R.F.P.S.; Professor of Medicine, Anderson's College Medical School ; Physician, Royal Infirmary; Lecturer in Clinical Medicine in the University, Glasgow. With Chapters on THE ELECTRO-CARDIOGRAPH, by W. T. RITCHIE, F.R.C.P., and THE OCULAR MANIFESTATIONS IN ARTERIO-SCLEROSIS, by ARTHUR J. BALLANTYNE, M.D., F.R.F.P.S. With 3 Coloured Plates and 199 Illustrations in the Text. $\mathrm{xx}+43^{8}$ pages. I5s. net. 


\section{Third Edition, Revised and EnLarged.}

\section{THE DIAGNOSIS OF NERVOUS DISEASES.}

By Purves Stewart, M.A., M.D. (Edin.), F.R.C.P., Physician to Out-Patients at the Westminster Hospital ; Joint Lecturer on Medicine in the Medical School; Physician to the West End Hospital for Nervous Diseases, and to the Royal National Orthopædic Hospital; Consulting Physician to the Central London Throat Hospital. vii +477 pages, with 2 full-page Plates and 225 Illustrations from Original Diagrams and Clinical Photographs. I5s. net.

"To practitioners unable to profit by post-graduate study, the careful descriptions, illustrated by the author's photographs, of the various methods of clinical investigation, the correct methods of eliciting the many superficial and deep reflexes, of performing lumbar puncture, of making an electro-diagnosis, and so on, and the significance of these phenomena in disease, will be simply invaluable. The whole book, which is beautifully illustrated and most carefully indexed, though in no way intended to replace the larger text-books already in use, will be found to be a most valuable supplement which we cannot too strongly commend to practitioners and students."British Medical Journal.

* Translations of this work in German and French have recently been published.

\section{A SYSTEM OF CLINICAL MEDICINE}

DEALING WITH THE DIAGNOSIS, PROGNOSIS, AND TREATMENT OF DISEASE.

By the late Thomas Dixon Savill, M.D. (Lond.). Fourth Edition, Revised by Agnes Savill, M.A., M.D. (Glas.), and others. xxviii +948 pages. With 4 Coloured Plates and I7o other Illustrations. 8 vo. 25s. net.

\section{EXTRACTS FROM PRESS NOTICES OF THE NEW EDITION.}

"May be regarded as thoroughly up to date."-British Medical Journal.

"Dr. Savill's book fully justifies its title, since its aims are to facilitate the clinical study ot disease, to assist in diagnosis, and to indicate the general lines of treatment. 'The new edition has been carefully revised, and remains as a notable contribution to the text-books of medicines, and a worthy memorial of its talented and lamented author."-Lancet.

"The best work on the subject of Clinical Medicine in the English language." -West London Medical Journal. 
NEW WORK BY DR. ROBERT SAUNDBY.

\section{URGENT SYMPTOMS IN MEDICAL PRACTICE.}

By Robert Saundby, M.D. (Edin.) ; Hon. LL.D. (St. Andrews and McGill); Hon. M.Sc. (Birm.); F.R.C.P. (Lond.); Hon. F.R.C.P. (Ireland); Professor of Medicine in the University of Birmingham; Consulting Physician to the General Hospital, Birmingham, to the Birmingham and Midland Counties Eye Hospital, and to the West Bromwich Hospital ; Ex-President, formerly President of Council, of the British Medical Association; Emeritus Senior President of the Royal Medical Society. $v+426$ pages. $7 \mathrm{~s} .6 \mathrm{~d}$. net.

\section{OLD AGE: ITS CARE AND TREAT- MENT IN HEALTH AND DISEASE}

By Robert Saundby, M.D. (Edin.); F.R.C.P. (Lond.); Professor of Medicine in the University of Birmingham, etc. vii +312 pages. Demy 8vo. 7s. 6d. net.

\section{WORKS BY DR. ROBERT HUTCHISON. FOOD AND THE PRINCIPLES OF DIETETICS.}

By Robert Hutchison, M.D. (Edin.), F.R.C.P., Physician to the London Hospital, and Assistant Physician to the Hospital for Sick Children, Great Ormond Street. New and Revised Edition. $\mathrm{xx}+6 \mathrm{I}_{5}$ pages, with Illustrations. I6s. net.

The British Medical Jourtal says :

(First Edition.) "Accepted as the leading work amongst English text-books on Dietetics."

(Latest Edition.) "The best work of its kind which has been published in recent years by anyone in this country."

\section{LECTURES ON DISEASES OF CHILDREN.}

Third Edition, revised and greatly enlarged. xii +404 pages, with many new Illustrations. Demy 8vo., cloth. Ios. 6 d. net.

"The whole of the book has been revised, and may be commended as a safe and convenient guide to the practitioner."- Lancet.

"In the second revised edition fresh chapters have been inserted. Each of these additions enhances the value and usefulness of the work. Dr. Hutchison possesses the happy gift of stating the main essentials of each complaint in a plain and lucid manner."-British Medical Journal. 


\section{A PRACTICAL HANDBOOK OF SURGICAL AFTER-TREATMENT.}

By Alan H. Todd, B.Sc., M.S. (Lond.); F.R.C.S. (Eng.); Surgical Registrar and Tutor, Guy's Hospital. xii $+25^{6}$ pages. Illustrated. Cloth. 4s. 6d. net.

Whether or not an operation is successful depends, not only upon the skill of the surgeon and the method of carrying out the operation, but upon the care which is subsequently bestowed upon the patient and the method of after-treatment employed.

Careful after-treatment will often bring through safely the most hopeless case, whilst neglect or lack of care will render void the result of the most skilful surgeon's efforts.

The Author has written an essentially practical book, giving clear and precise instructions for the after-treatment of every kind of surgical case. The book will be of the greatest service to house-surgeons and dressers, and also to sisters, nurses, and everyone who has anything to do with the aftertreatment of a modern surgical case.

\section{SURGICAL MATERIALS AND THEIR USES.}

By Alexander MacLennan, M.B., C.M. (Glas.); Visiting Surgeon, Glasgow Royal Hospital for Sick Children; Assistant Surgeon, Western Infirmary, Glasgow; etc., etc. viii +252 pages. With 277 Diagrams and Illustrations. Cloth. 4s. 6d. net. .

"This book is divided into six sections which deal with bandaging, splints, dressings, antiseptics and asepsis, sutures and ligatures, and instruments. It is the sort of manual which should be in the hands of every student who is beginning surgical hospital work, and we can recommend it as a vade-mecum to all such. It is well and clearly written, copiously illustrated, and deserves-as we have no doubt it. will obtain-a large circulation."-Liverpool Medico. Chirurgical Journal.

\section{MODERN AN/ESTHETICS.}

By J. Frederick W. Silk, M.D. (Lond.); Senior Anæsthetist and Lecturer on Anæsthetics, King's College Hospital, etc., etc. xii +200 pages. Illustrated. Cloth. 3s. 6 d. net.

The object of the author has been to describe in detail those procedures which he has found most useful in practice. These descriptions are preceded by a short account of the phenomena of anæsthesia, and are followed by a rather fuller reference to the difficulties met with, and their treatment, both in simple and exceptional operations. 


\section{THE CARRIER PROBLEM IN INFECTIOUS DISEASE.}

With particular reference to Enteric Fever, Diphtheria, CerebroSpinal Meningitis, Bacillary Dysentery and Cholera.

By J. C. G. Ledingham, M.B., D.Sc., Chief Bacteriologist, Lister Institute of Preventive Medicine, London; Reader in Bacteriology, University of London; and J. A. ARKWRIGHT, M.D., Assistant Bacteriologist, Lister Institute of Preventive Medicine, London. vii + 319 pages. I2s. 6 d. net.

The object of the authors, who have made an elaborate study of the subject, has been to present in as complete a form as possible the present state of knowledge with regard to the rôle played in inf utious diseases by human carriers-individuals outwardly healthy, or at most suffering from some trivial chronic complaint. To epidemiologists and public health authorities, as well as to bacteriologists, the book will strongly appeal.

** ${ }^{*}$ The above is the seventh volume in the Series of INTERNATIONAL MEDiCAL MONOGRAPHS. Edited by Leonard Hill, M.B., F.R.S., Director of Applied Physiology and Hygiene, Medical Research Committee ; and William Bulloch, M.D., Professor of Bacteriology, University, London.

In this series will be found volumes dealing with subjects of exceptional interest and importance, giving in a concise form the results of the leading investigators in special branches of medical science throughout the world. Attention also is given to the practical application of the results of scientific research to the treatment of disease.

Full descriptive circular of the series will be sent free on request.

WORKS BY DR. R. W. LEFTWICH. Second Edition, Thoroughly Revised.

\section{A POCKET-BOOK OF TREATMENT}

By Ralph Winnington Leftwich, M.D., Author of "Tabular Diagnosis," etc. viii $+34^{8}$ pages. Flexible binding, with wallet flap. 6s. net.

"As a handy guide to treatment, and as a useful pocket formulary, we can unhesitatingly recommend Dr. Leftwich's book. It is full of practical and valuable hints." - The Practitioner.

\section{TABULAR DIAGNOSIS}

\section{AN AID TO THE RAPID DIFFERENTIAL DIAGNOSIS OF DISEASE.}

By Ralph Winnington Leftwich, M.D., Late Assistant Physician to the East London Children's Hospital. vi +359 pages. $7 \mathrm{~s}$. $6 \mathrm{~d}$. net.

"This book is written for the busy practitioner, and is designed to help him in diagnosis. Such a volume on its present scale is certainly a new departure in medical literature, and should serve a useful purpose. It contains a great deal of information, which has been carefully compiled and admirably arranged for the purpose of easy reference. For the highly important purpose of differential diagnosis it is certainly a much more rapid guide than the ordinary text.book."

Edinburgh Medical Journal. 


\section{MILITARY HYGIENE AND SANITATION.}

By Colonel Charles H. Melville, M.B. (Edin.), D.P.H.; Royal Army Medical Corps; late Sanitary Officer, Army Headquarters, India ; late Secretary and Expert in Sanitation, Army Medical Advisory Board, War Office; Professor of Hygiene, Royal Army Medical College; Member of Council, Royal Sanitary Institute. Illustrated. vii +4 I 8 pages. Demy 8 vo. I 2s. 6 d. net.

A book "to enable the Army Medical Officer to maintain the soldier clear of Hospital and possessing the fullest measure of his physical powers."-Army and Navy Gazette.

"In our opinion this is quite the best work which has appeared on Military Hygiene and Sanitation. All through the book Colonel Melville gives sound practical advice-based on many years of practical experience in army sanitary work. We can most strongly recommend this work to all Medical Officers of the Regular or Territorial Forces, but more especially to those who are commencing a military medical career."-British Medical Journal.

\section{THE SANITARY OFFICER'S HANDBOOK OF PRACTICAL HYGIENE.}

By W. W. O. Beveridge, D.S.O., Lieut.-Col. R.A.M.C., M.B., C.M. (Edin.), D.P.H. (Camb.); Fellow of the Chemical Society; Analyst to the Army Advisory Board; and C. F. Wanhill, Major R.A.M.C., M.R.C.S. (Eng.), L.R.C.P. (Lond.), D.P.H. (Conjoint Board of Eng.); late Assistant Professor of Hygiene, Royal Army Medical College. Second Edition, Revised and Enlarged. viii +236 pages. Interleaved with blank paper for Notes. Crown 8vo. 6s. net.

"It deserves inclusion in the library of every Medical Officer in the service."-Lancet.

Human Embryology and Morphology. By Arthur Keith, M.D. Third Edition. I5s. net.

Practical Anatomy. By F. G. Parsons, F.R.C.S., and William Wright, F.R.C.S. Two Volumes. 8s. 6d. net each.

Practical Physiology. By M. S. Pembrey, M.A., M.D., and others. Third Edition. I4s. net.

Further Advances in Physiology. Edited by Prof. LEONARD Hill, F.R.S. I5s. net.

Internal Secretion and the Ductless Glands. By Swale Vincent, M.D. r2s. 6d. net.

Manual of Pharmacology. Fourth Edition. By W. E. Dixon. I5s. net.

Full descriptive Catalogue containing particulars of Mr. Edward Arnold's publications in the various branches of Medicine and Surgery will be sent post free on request. 



\section{GENERAL LIBRARY}

UNIVERSITY OF CALIFORNIA-BERKELEY

RETURN TO DESK FROM WHICH BORROWED

This book is due on the last date stamped below, or on the date to which renewed.

Renewed books are subject to immediate recall.

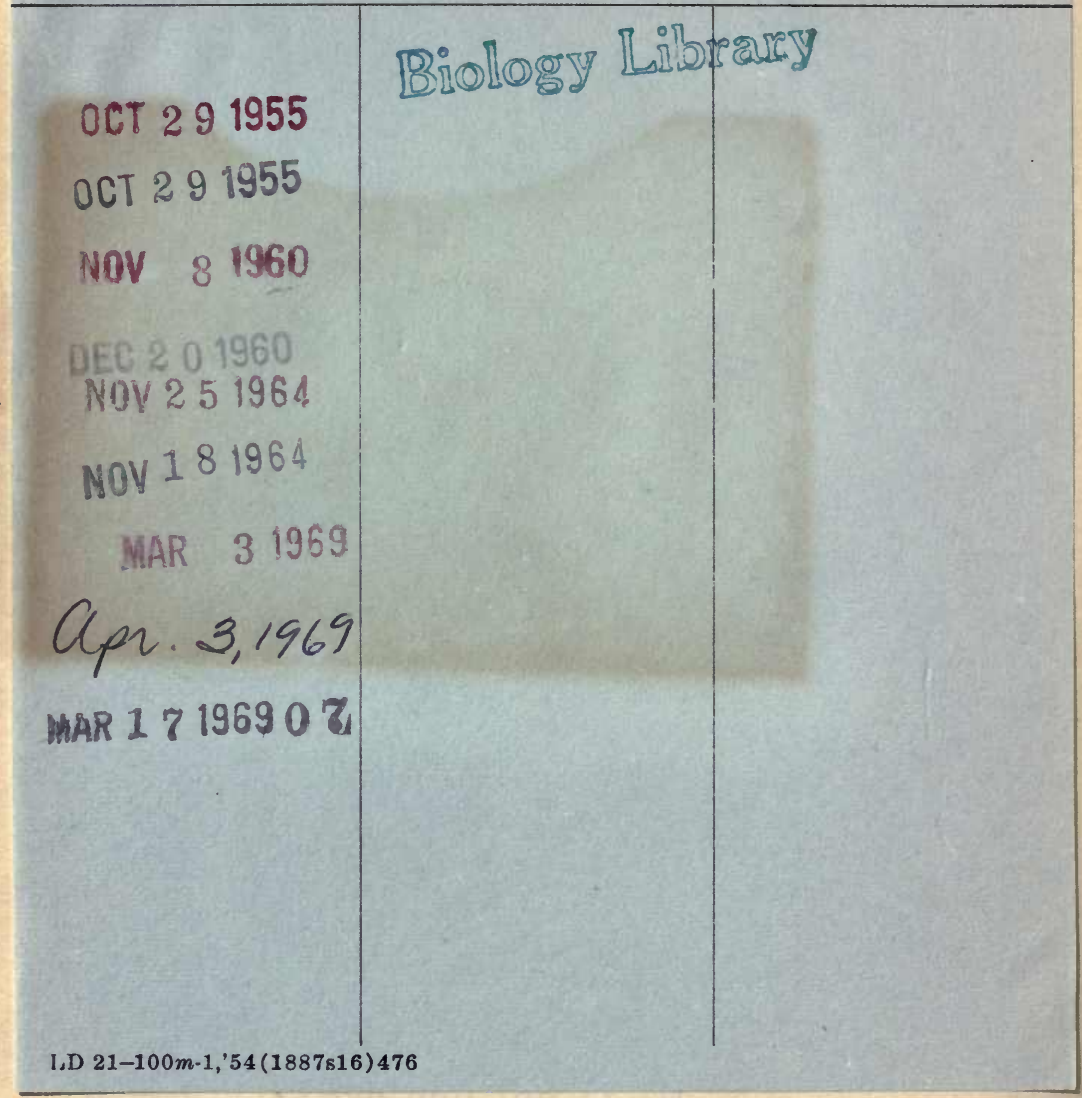



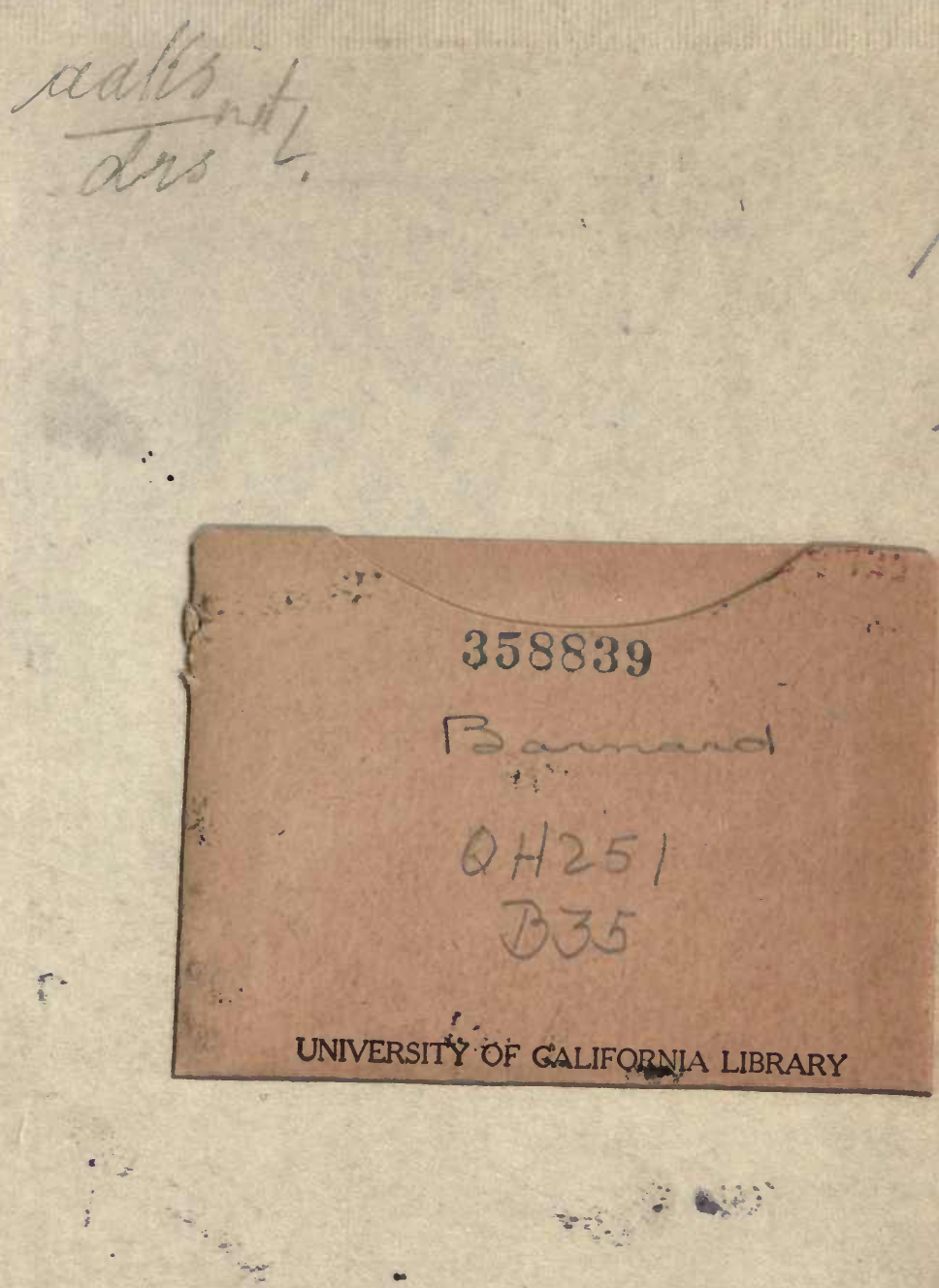

$$
\text { म. }
$$


35n

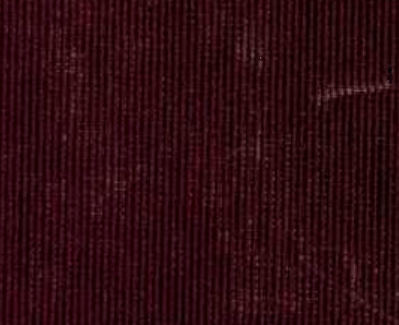

